\title{
Greece: First and Second Reviews Under the Extended Arrangement Under the Extended Fund Facility, Request for Waiver of Applicability, Modification of Performance Criteria, and Rephasing of Access-Staff Report; Staff Supplement; Press Release on the Executive Board Discussion; and Statement by the Executive Director for Greece.
}

In the context of the first and second reviews under the Extended Arrangement under the Extended Fund Facility, request for waiver of applicability, modification of performance criteria, and rephasing of access, the following documents have been released and are included in this package:

- $\quad$ The staff report for the first and second reviews under the Extended Arrangement under the Extended Fund Facility, request for waiver of applicability, modification of performance criteria, and rephasing of access, prepared by a staff team of the IMF, following discussions that ended on October 16, 2012, with the officials of Greece on economic developments and policies. Based on information available at the time of these discussions, the staff report was completed on December 21, 2012. The views expressed in the staff report are those of the staff team and do not necessarily reflect the views of the Executive Board of the IMF.

- $\quad$ A staff supplement of January 15, 2013 updating information on recent developments.

- $\quad$ A Press Release summarizing the views of the Executive Board as expressed during its January 16, 2013 discussion of the staff report that completed the request and/or review.

- $\quad$ A statement by the Executive Director for Greece.

The documents listed below have been or will be separately released.

Letter of Intent sent to the IMF by the authorities of Greece*

Memorandum of Economic and Financial Policies by the authorities of Greece*

Technical Memorandum of Understanding*

Letter of Intent to the European Commission and the European Central Bank*

Memorandum of Understanding on Specific Economic Policy Conditionality*

*Also included in Staff Report

The policy of publication of staff reports and other documents allows for the deletion of market-sensitive information.

Copies of this report are available to the public from

International Monetary Fund • Publication Services

$70019^{\text {th }}$ Street, N.W. • Washington, D.C. 20431

Telephone: (202) 623-7430 • Telefax: (202) 623-7201

E-mail: publications@imf.org Internet: http://www.imf.org

\section{International Monetary Fund \\ Washington, D.C.}




\section{INTERNATIONAL MONETARY FUND}

\section{GREECE}

December 21, 2012

FIRST AND SECOND REVIEWS UNDER THE EXTENDED

ARRANGEMENT UNDER THE EXTENDED FUND FACILITY,

REQUEST FOR WAIVER OF APPLICABILITY, MODIFICATION OF

PERFORMANCE CRITERIA, AND REPHASING OF ACCESS

\section{EXECUTIVE SUMMARY}

Extended Arrangement. On March 15, 2012, the Executive Board approved a four-year Extended Arrangement under the Extended Fund Facility in the amount equivalent to SDR 23.79 billion (2,159 percent of quota; $€ 28$ billion) with equally phased purchases of SDR 1.4 billion (about $€ 1.65$ billion). The first purchase was made at the time of approval of the arrangement; the second and third purchases in the amount equivalent to SDR 2.8 billion (€3.3 billion) are proposed to be released on the completion of the combined first and second reviews. Euro area countries disbursed $€ 73.9$ billion shortly after program approval (of $€ 144.6$ billion committed) and about a further $€ 34.3$ billion in mid-December 2012.

Recent Developments. The program ran off track due to a severe political crisis. The extended election period effectively put on hold the implementation of macro-structural reforms and the privatization process and fiscal-institutional reforms also came to a halt. As a result, several performance criteria, indicative targets and structural benchmarks were not observed. Time was required to restart reforms and bring the program back on track, and to reach understandings with the authorities and Greece's European partners on measures to place debt on a sustainable trajectory. The delays have had macro repercussions. The program anticipated a weak economy in 2012, as Greece continued to adjust through recessionary rather than productivity-boosting channels. GDP has now been undershooting program projections due to weaker confidence and tight liquidity conditions, alongside weakness in export markets. It is now projected to contract by 6 percent this year and 41/4 percent in 2013. The deeper recession has accelerated current account adjustment, and internal devaluation is also now gaining pace. The fiscal position continues to improve, although domestic spending arrears have increased.

Program policies. Understandings have been reached with the new government on a fully recalibrated program. Program policies were modified to deal with stronger macroeconomic headwinds and to better reflect observed implementation capacity. The fiscal adjustment path has been lengthened by two years to 2016 to give more time to reach the primary balance target. Privatization targets have also been adjusted downward to reflect weak market conditions and the need to prepare assets. The authorities specified the adjustment measures necessary to 
close the fiscal gap through 2014. They have also taken measures to liberalize product markets, and to lower nonwage costs, to help stem rising unemployment and falling real wages. In the financial sector, the bank recapitalization process has advanced, and oversight and governance will be strengthened. Fiscal institutional reforms to strengthen the tax administration and public financial management have been put back on track, although are behind schedule. In all areas, key measures have been adopted as prior actions for the first and second reviews.

Program financing. The new government also had to reach understandings with its European partners on a revised financing framework. The updated DSA showed that further debt relief was needed to ensure that Greece's debt is sustainable. Greece's European partners have recognized this and have, as a first step, agreed to lower the interest rate on the Greek Loan Facility and to lengthen maturities on all of their lending, and to transfer profits earned by the ECB on Greek debt back to Greece. They also agreed to bring forward program financing to support a buyback of recently-restructured Greek government bonds, which was completed in December 2012. These measures are projected to bring debt to 128 percent of GDP by 2020. Thus Greece's partners also committed to take further actions, once Greece has further advanced with its fiscal adjustment, to bring debt down to 124 percent of GDP by 2020 and substantially below 110 percent of GDP in 2022. Finally, Greece's European partners agreed to provide roughly $€ 26$ billion in additional financing for the period 2012-16, to help cover projected financing gaps. With the relief, a gap of $€ 51 / 2-91 / 2$ billion remains during $2015-16$, but they also reiterated their commitment to support Greece as necessary during and beyond the program, provided Greece implements the program. Timely delivery of Greece's European partners' undertakings on debt relief and financing is crucial for program success.

Discussions. See the Fund Relations Appendix 
Approved By

Reza Moghadam and Lorenzo Giorgianni
Discussions for the first and second reviews under the Extended Arrangement were held during July 3-11, July 24-August 5, September 5-20, and October 1-16. The mission met with the Prime Minister, Minister of Finance, Governor of the Bank of Greece, and other Cabinet Ministers; and staff in these and other ministries. The mission also met private banks, think tanks, and employer associations. The staff team comprised P. Thomsen (head), M. Flanagan, W. McGrew, H. Floerkemeier, G. Gottlieb, N. Hobdari, W. Maliszewski, and M. Shamloo (EUR); G. Palomba, and I. Petrova (FAD); S. Lanau (SPR); G. Mitchell Casselle, L. Cortavarria, N. Jassaud, and N. Saker (MCM); and G. Esposito and Y. Liu (LEG). B. Traa, S. Eble, G. Gatopoulos, and M. Athanasopoulou (IMF resident representative office) assisted the mission.

\section{CONTENTS}

BACKGROUND

DISCUSSIONS

A. The Macroeconomic Framework 12

B. Economic Policies 15

DEBT SUSTAINABILITY AND FINANCING 30

\section{TABLES}

1. Selected Economic Indicators, 2007-13

2. Greece: General Government: Statement of Operations (GFSM 2001, flows), 2008-11

3. Financial Balance Sheet (GFSM 2001, stocks), 2008-11

4. Modified General Government Cash Balance, 2012-16

5. Monetary Survey, 2007-12

6. Core Set of Financial Soundness Indicators for Deposit-Taking Institutions 49

7. Implementation of Structural Reforms

8. Greece: Status of Fiscal Structural Reforms 51

9. Medium-Term Macro Framework, 2012-20

11. Monetary Financial Institutions (excl. BoG)—Uses and Sources, 2006-16 54 
13. Privatization Process, 2012-14 _ 56

14. General Government Operations, 2012-16__ 57

15 Authorities' Measures to Meet the 2013-14 Targets ___ 58

16. Income Tax Reform__ 59

17. Revenue Collection Process_-Issues and Actions ___ 60

18. Spending Process-Issues and Actions __ 61

19. General Government Financing Requirements, 2010-16___ 62

20. External Financing Requirements and Sources, 2010-17___ 63

22. Indicators of Fund Credit, 2012-26___ 65

\section{FIGURES}

1. Selected Economic Indicators, 2005-12 __ 66

2. Inflation Developments, 2005-12_ 67

3. Competitiveness Indicators, 2005-12___ 68

4. Labor Market Developments, 2007-12_ 69

5. Budget Execution, 2012 _ 70

6. Balance of Payments Developments, 2010-12 _ 71

7. Money and Banking Developments, 2007-12 _ 72

8. Revised Macro Projections, 2008-16 __ 73

9. International Comparison of Macro Projections__ 74

10. Financial Indicators, 2007-12___ 75

11. Revenue and Expenditure Trends, 2000-16__ 76

\section{BOXES}

1. Increasing Revenue Collection: Progress and Bottlenecks___ 77

2. Greece as a Source of Contagion___ 78

3. The Macroeconomic Impact of a Debt Overhang ___ 81

4: Measures Taken to Address Greek Debt Burden ___ 84

\section{APPENDIXES}

I. Debt Sustainability Analysis___ 85

II. Fund Relations__ 97

III. Letter of Intent 101

IV. Memorandum of Economic and Financial Policies___ 104

V. Technical Memorandum of Understanding ___ 149

VI. Letter of Intent to the European Commission and the European Central Bank ___ 163

VII. Memorandum of Understanding on Specific Economic Policy Conditionality___ 166 


\section{BACKGROUND}

1. Program review discussions were delayed by two rounds of national elections, which revealed a marked shift in support for the program. At program inception, hopes were high that a greater consensus would emerge behind program policies since a grand coalition had assumed power, bringing the main opposition party into the fold. The elections represented a significant political shake-up, with the radical left party Syriza coming second, after campaigning strongly against program policies. Extremist parties also gained in popularity. Nonetheless, a new coalition emerged supporting the program. The new government, headed by PM Samaras, is supported by the conservative New Democracy (the largest party in the parliament), center-left PASOK, and the Democratic Left party.

2. Implementation of the program stalled in several areas while elections unfolded. EndSeptember 2012 quantitative fiscal targets were mostly met, with the exception of the performance criterion and indicative target on expenditure arrears, and the performance criterion on government guarantees. Indicative targets for privatization receipts for end-September 2012 were also missed (MEFP Table 1a). The stalemate during the election period and escalating social tensions took a deeper toll on implementation of structural reforms. The structural benchmarks on reducing the tax wedge and on meeting the performance indicators for revenue administration and public financial management were not observed, while benchmarks on preparing various inputs into the fiscal adjustment strategy were only observed with considerable delay (MEFP Table 2). In the end, the discussions extended into the period for the third review.

\section{RECENT DEVELOPMENTS}

3. Greece's deep recession has continued unabated, with the economy struggling to gain traction against domestic political instability and weak external conditions (Table 1, Figure 1). GDP contracted by $6 \frac{1}{2} 2$ percent $y-0 n-y$ in the first three quarters of the year. The program had anticipated a deep contraction, as Greece continued to adjust through recessionary rather than productivity-boosting channels. However, rising uncertainty, tighter liquidity conditions (seriously aggravated by an accumulation of government arrears), and falling disposable income (due to faster-than-expected cuts in wages) have combined to accelerate the decline in domestic demand. Overall, the cumulative output decline has now reached about 191/2 percent (including the impact of recently announced GDP revisions, which shaved over 11/2 percent off reported growth in 2010-11). 


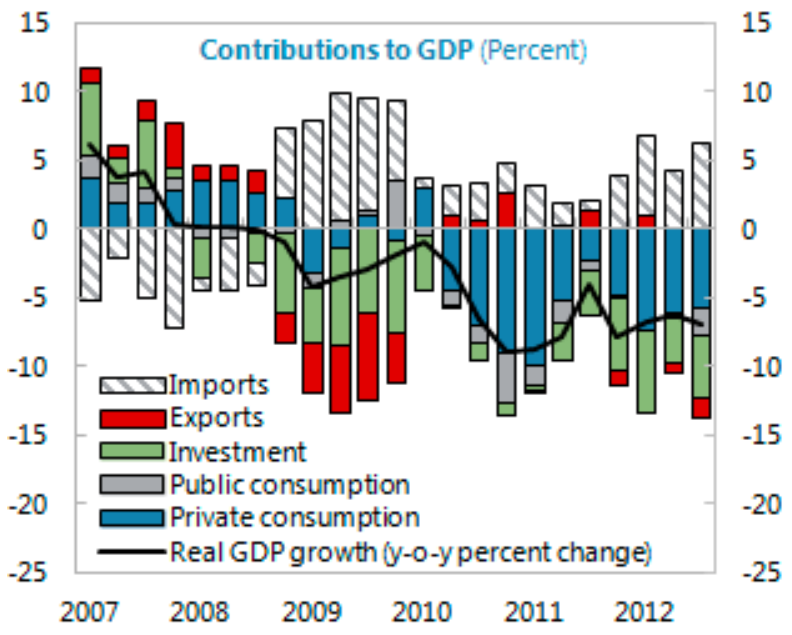

Sources: Elstat; and IMF staff calculations.

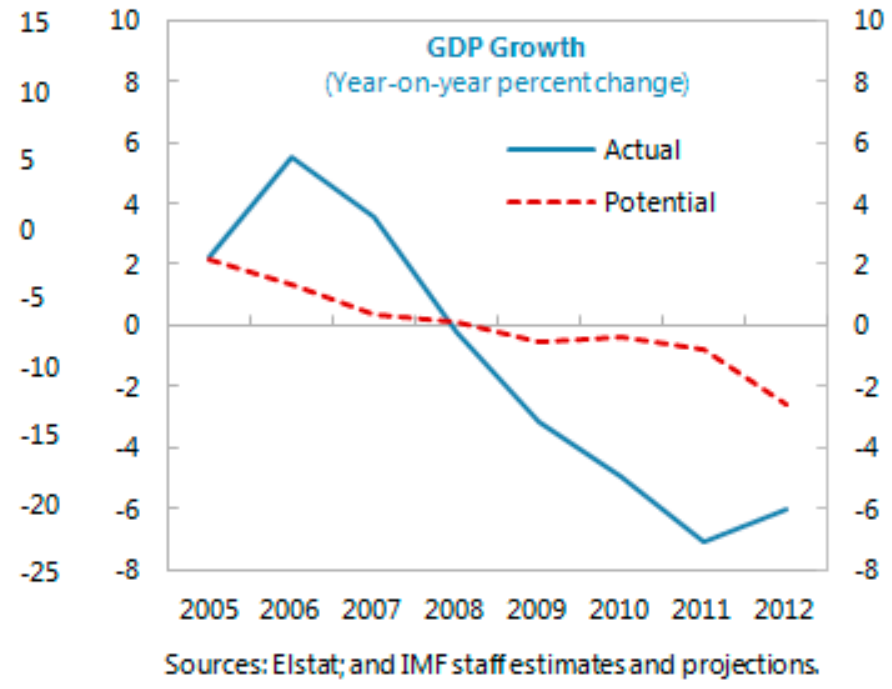

4. Stronger internal devaluation is now underway, reflecting the interaction of labor market liberalization with the already very weak labor market (Table 1, Figure 2-4).

- Wages have begun to adjust at a much faster pace. Nominal wages in the economy declined by an average of 71/2 percent y-o-y in Q2. Firm-level and individual wage agreements-both instruments which are growing in share facilitated by recently introduced institutional reforms - saw even larger declines of about 20 percent. Moreover, the most recently-signed contracts and survey indicators suggest that this trend is continuing. Greek wage changes now exceed what would simply be predicted based on cumulative GDP movements. In a very soft labor market-unemployment reached 26 percent at endOctober-the far-reaching reforms implemented in late 2011 and early 2012 appear to have paved the way for needed adjustment. The ULC-based REER has now fallen by 14 percent through September from its peak in 2009.
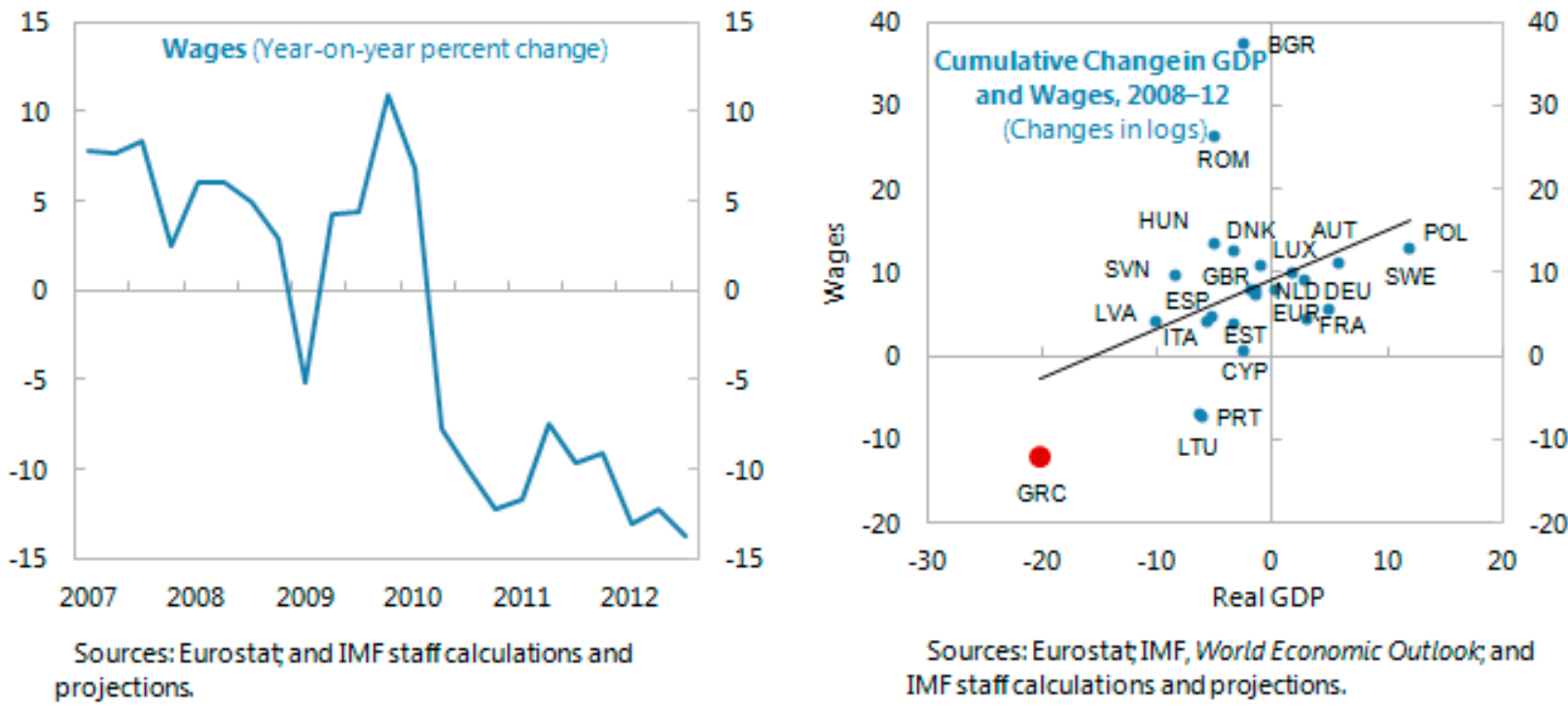

Sources: Eurostat IMF, World Economic Outlook; and IMF staff calculations and projections. 
- Prices are also adjusting, but not as quickly as wages. Headline HICP inflation, at 0.4 percent $y$-on-y in November, is firmly below the euro area average. Indirect taxes, administered prices and unprocessed food and energy prices have been the main contributors to inflation during the recession. With these components stripped out, prices have been falling since 2010. The GDP deflator has already turned negative in the first three quarters of 2012 on the back of falling wages and a weaker terms-of-trade. The improvement in the CPI-based REER is a more modest 6 percent.

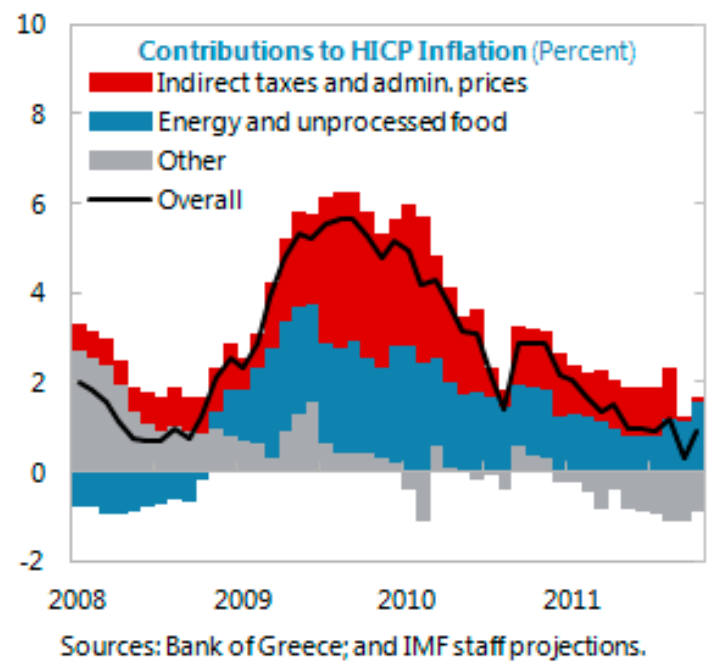

5. Fiscal and external imbalances are correcting:

The fiscal position continues to improve via strong structural adjustment (Tables 2-4, Figure 5). The trailing 12-month cash central government primary balance was close to zero through end-October (compared to a deficit of 3 percent of GDP one year ago). State revenues have trended below program, reflecting macro headwinds and collection problems, but spending has been constrained well below program limits as the authorities confronted a lack of finance. Cash shortages did contribute to a buildup of arrears in 2012 (some 3/4 percent of GDP, bringing the stock to almost 5 percent of GDP at end-October), but the accrual adjustment has also been running slightly ahead of plans. Still, some important underlying expenditure policy reforms have lagged, most notably efforts to make staff reductions more targeted (only 200 employees were placed in the labor reserve

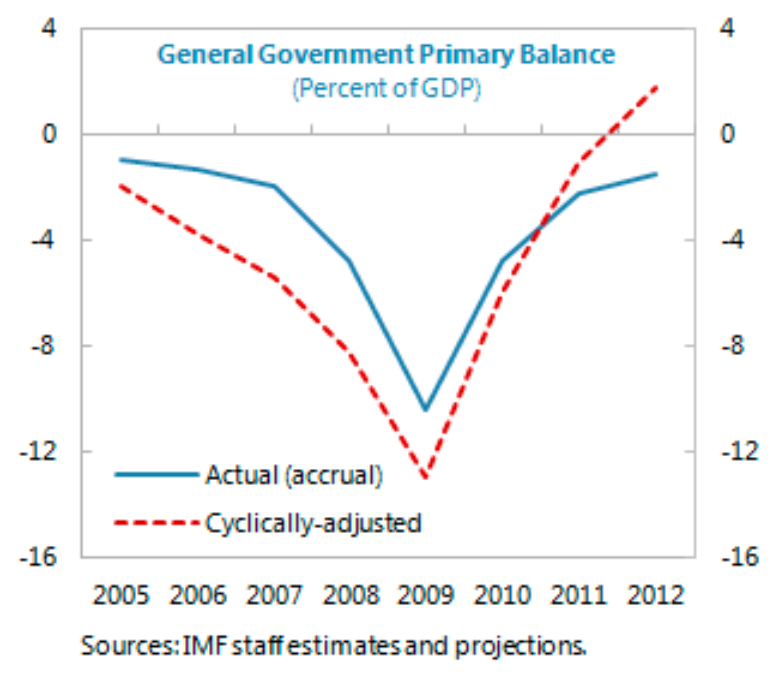
during 2012, well short of the end-2012 target of 15,000). 
- Current account adjustment has accelerated, notwithstanding slow progress with structural reforms, reflecting intensifying recessionary channels (Table 1, Figure 6). The overall magnitude of adjustment over 2008-11 has been relatively small compared to demand and output losses during this period. This suggests that slow progress in addressing structural rigidities and market distortions have restrained a shift of resources from the nontradable to the tradable sector. Still, the trailing 12-month deficit reached 5 percent of GDP through end-September (compared with 10 percent during the same period in 2010-11). The deep recession is driving a steep contraction of imports, helping the trade balance. The overall current account also benefitted from the March 2012 debt exchange, which improved the income account balance by $1 \frac{1}{2}$ percent of GDP.

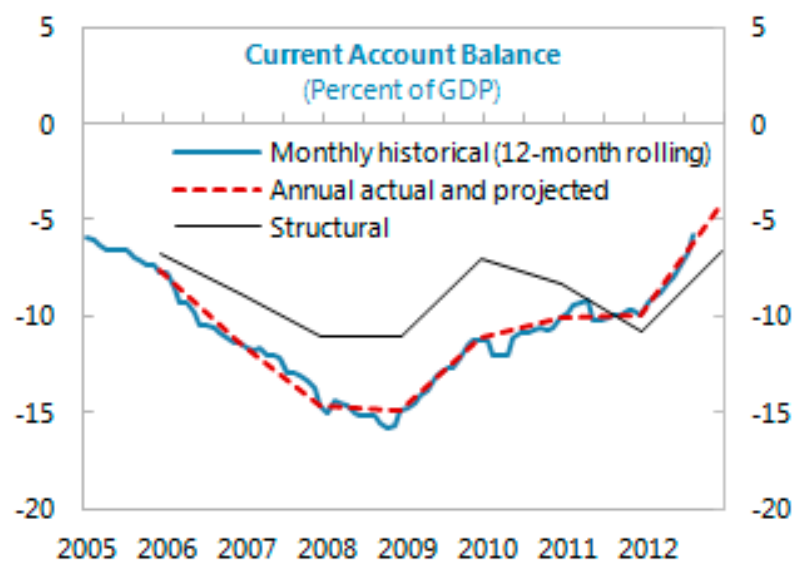

Sources: Bank of Greece; and IMF staff projections.

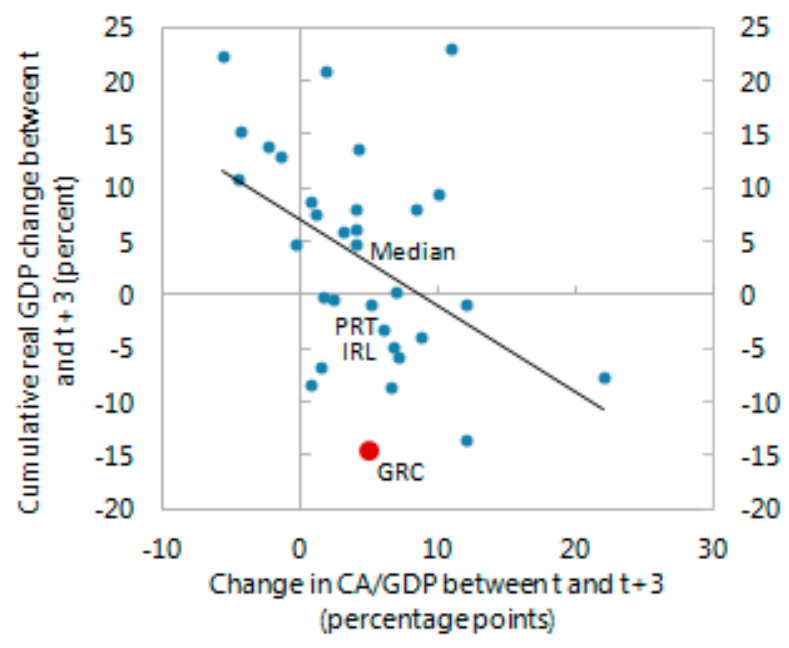

Sources:IMF, World Economic Outlook and IMF staff calculations.

6. Pressure on the banking system has been intense, but program support has prevented a serious crisis (Table 5 and 6; Figure 7):

- Deposits have been volatile and asset performance continues to deteriorate. Election-

related uncertainty accelerated the outflow of private sector deposits to $€ 15.9$ billion in May and June 2012, and while there has been a reversal since, total private sector deposits as of end-November are still 10.6 percent lower than at the end of 2011. On the asset side, NPLs (including restructured loans) continue to rise, reaching 23.8 percent of total loans in Q1, an increase of almost 10.5 percentage points from end-2010. Preliminary Q2 data for the four largest banks shows a further rise to 24 percent. Banks have struggled with their

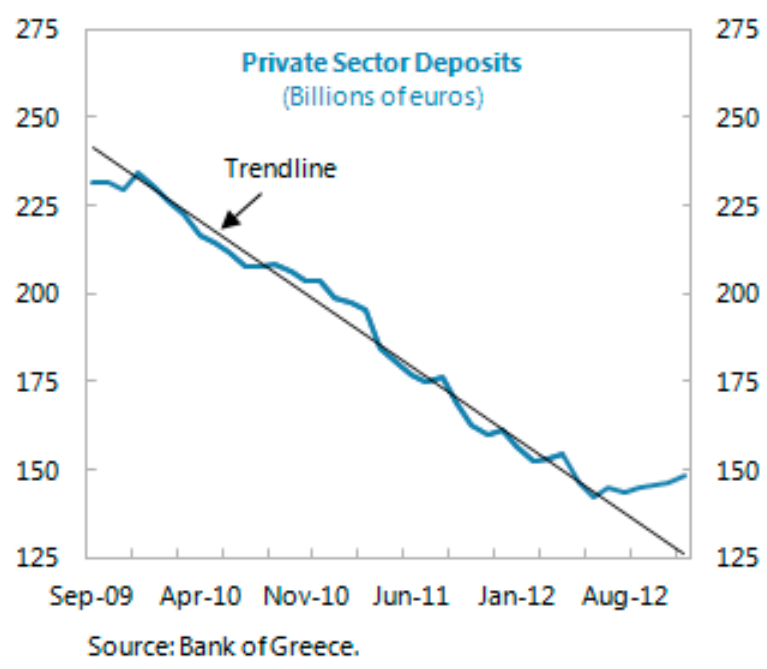


capital adequacy ratios since the PSI exercise. Moreover, provisioning has lagged behind NPLs, with the coverage ratio declining to 41.4 percent in Q1 from 45.2 percent at end-2011.

Banks have responded to these pressures by increased recourse to exceptional liquidity support, deleveraging, and mergers to cut costs. As of mid-December, central bank funding amounted to $€ 129$ billion, up from $€ 121$ billion at end-2011. With the ECB having disqualified most Greek banks from accessing the monetary policy operations window (due to capital adequacy ratios below the minimum requirement), the funding comes almost entirely in the form of more expensive ELA. This increase in costs, together with falling demand for credit and tightened lending standards, has contributed to accelerated deleveraging. Private sector credit declined by almost 5 percent $y$-on-y in November. The system is also consolidating to lower costs. Two smaller banks were acquired by two core banks, and merger steps between two large banks, NBG and EFG, are now underway.

The government has supported banks eligible for public aid with bridge recapitalization and has also stepped up its resolution work. The BoG completed a strategic assessment of the banking sector in March 2012 and identified four "core" banks as viable based on regulatory criteria (regulatory ratios, asset quality and governance) and an assessment of their business fundamentals (commercial strength, profitability, risk management). In June 2012 , the HFSF provided $€ 18$ billion in 'bridge capital' to these core banks in order to bring their CAR to 8 percent. (The private sector has retained management control, pending an opportunity to raise capital). In another step towards restructuring of the system, the largest public bank ATE was resolved in July 2012 through a P\&A transaction by Piraeus Bank with upfront costs for the HFSF of $€ 7.2$ billion.

\section{The structural transformation of Greece's economy continues to proceed at a slow} pace (outside of the labor market), and this is making Greece's adjustment more costly

(Table 7). The authorities made some progress right after approval of the Extended Arrangement, including adoption of legislation that simplified fast-track investment licensing and reduced red tape for exporters, and steps to improve the efficiency of court proceedings and implement licensing laws. The World Bank's Doing Business Indicator indeed now places Greece 11 positions higher than last year, based on improvements in the ease of starting a business, protecting investors, and facilitating external trade. But Greece's overall performance in the Doing Business Indicator remains well behind EU best

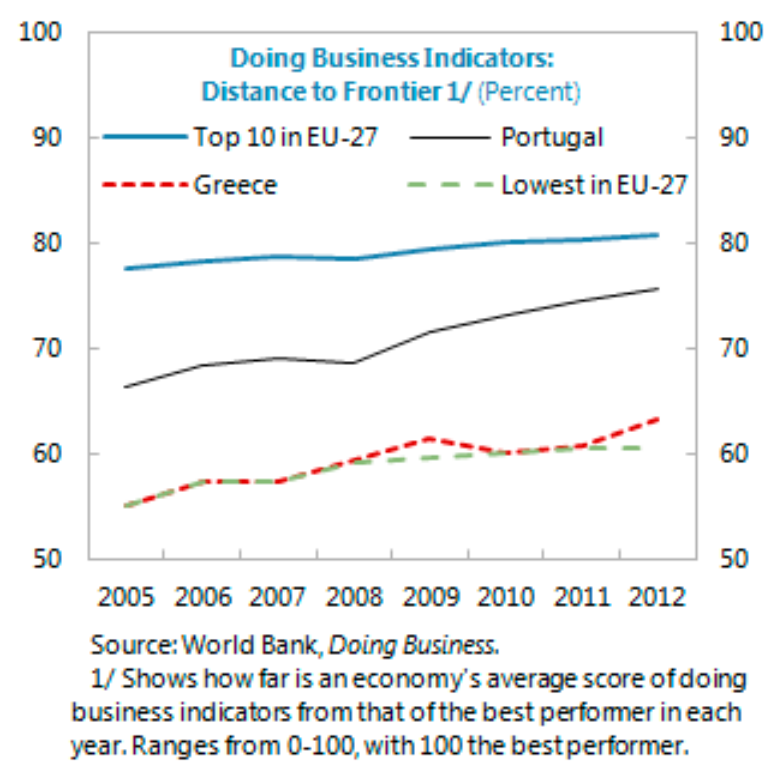


practice. And the extended election period effectively put on hold reforms aimed at liberalizing product and service markets (e.g., the regulated professions). Progress on judicial reforms (especially on reducing court case backlogs) also remained well behind schedule.

8. Privatization targets have been missed by a wide margin. Uncertainty about the election outcome and Greece's financial stability, and the absence of the Board of the Hellenic Republic Asset Development Fund (HRADF) for several months, brought the sale of assets to a complete halt (the full year 2012 target was set at $€ 3.2$ billion). Nevertheless, as part of a list of 78 priority actions, the HRADF has made progress in recent months to advance the preparation of assets, including state aid clearance, unblocking the sale of "golden shares" (mostly in utility companies), launching a tender for the gas company DEPA and betting firm OPAP, identifying a portfolio of 3,000 commercially viable real estate assets, specifying 40 real estate assets slated for near-term privatization, and clearing up regulatory provisions. However, political resistance remains strong, and parliament passed an amendment to the privatization law to allow the Parliamentary Economic Committee to provide recommendations before any privatization transaction.

\section{Institutional reforms continued to disappoint during 2012, again complicating overall adjustment efforts (Table 8 ).}

- Tax administration reform stalled (Box 1). Few efforts have been made over the last 10 months to address the problems of fragmentation, understaffing, inadequate operating procedures and weak internal controls. In part, this reflects a leadership vacuum (there has been no Secretary General for the last 10 months), but it also reflects deep seated bureaucratic resistance. Tax collection and tax payment discipline has suffered. The tax administration has fallen well short of its performance targets, in areas such as the number of large taxpayer and high wealth individual audits and debt collection. EndDecember 2012 targets in these areas, set at

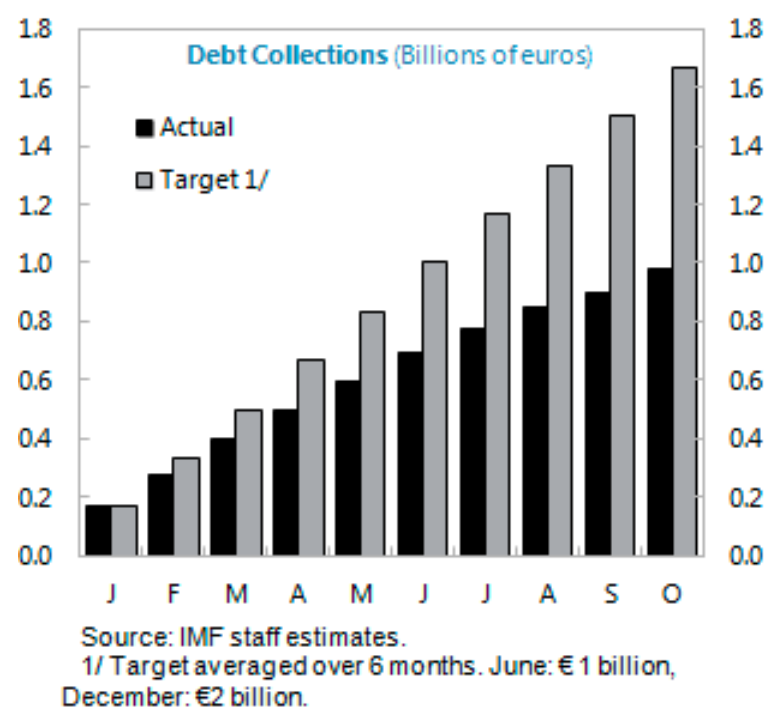
program approval, will almost certainly be missed.

- Public financial management reform has also slowed down. Commitment registers were set up in around 70 percent of general government entities, although there remained some major holdouts, including the health social security fund, EOPYY (which was established in January, but started reporting only in October). Unfortunately, the slow pace of posting qualified staff has reduced the effectiveness of commitment controls in controlling spending and arrears. 


\section{STRATEGY}

10. Greece is making progress, but remains some distance from achieving its program objectives. Overall, the economy continues to adjust through recessionary channels and at a great loss to output. Reforms to boost supply and productivity are still lagging behind schedule and efforts to alleviate the public debt overhang continue to run against headwinds. Against this backdrop, while Greece has made significant progress with fiscal adjustment, some 6 percent of GDP additional primary adjustment is still needed to reach the program target. Similarly, despite considerable progress with external adjustment and cost competitiveness, there is still some distance to go, with only about half of the estimated 20-30 percent pre-program real effective exchange rate overvaluation eliminated. Finally, while a financial sector crisis has been averted, and banks are now closing in on full recapitalization, a major restructuring effort lies ahead before financial stability is assured.

11. Against this backdrop, the present reviews also had to confront major new challenges. First, the sharp loss of political support for the program and reconstitution of a determined opposition significantly weakened the ability of Greece to undertake the type of ambitious frontloaded policies built into the EFF program. Second, the deepening recession created further headwinds for fiscal adjustment, increased the burden of Greece's debt, and raised substantially the probability that Greece would get stuck in a weak-confidence, high-debt, low-growth trap. Staff's assessment at the outset of the review discussions in June was indeed that downside risks had materialized, leaving the unsustainable "alternative" debt path under the Extended Arrangement as the new baseline outlook.

12. The program strategy remains to help Greece address its external adjustment and debt overhang challenges within the euro area. The alternative of euro exit would quickly correct exchange rate overvaluation and eventually reduce the debt overhang through default and a subsequent comprehensive restructuring, but would likely lead to severe economic disruption. ${ }^{1}$ The authorities, concerned about the domestic costs and risks to social cohesion that would follow such events, are determined to stay in the euro area. Greece's European partners, in turn, remain concerned about international spillovers, especially market perceptions about the euro area's stability. Simulations suggest that while the risk of contagion has been reduced, the impact of Greek exit on euro area GDP would still be significant (Box 2). On balance, staff assesses that adjustment within the euro area would be the best course for both Greece and Europe, and the focus was thus on encouraging the two sides to do what would be necessary to make this work, in light of recent developments.

13. Understandings were reached with the Greek authorities on revised policies to help Greece reach its objectives within the euro area while managing its significant challenges. In

\footnotetext{
${ }^{1}$ See Box 2 in the staff report for the request for an Extended Arrangement (IMF Country Report No. 12/57).
} 
particular: (i) the macro outlook was adjusted to reflect the deeper recession, tight financing conditions and the worse external outlook; (ii) macro-structural reforms were given additional emphasis, in a renewed effort to boost external rebalancing and help reduce the output cost of fiscal adjustment; (iii) privatization targets were revised down, reflecting poor market conditions, but also revised bottom-up estimates and an adjusted timeline for what will be a complex process, in the face of strong resistance from vested interests; and (iv) fiscal adjustment was extended by two years, to help cushion its impact on the economy and avoid undermining social cohesion. The section on Discussions discusses the changes in detail.

\section{The Greek authorities also reached understandings with their European partners on a} financing package that delivers debt sustainability. With the agreed changes in the macro-policy framework, the debt trajectory rose notably above the original program path, suggesting that confidence, investment, and growth would all be more suppressed than assumed, and thus that the whole framework would not be sustainable. Greece's European partners agreed to address this debt overhang by bringing forward program financing to support a debt buyback from the private sector, and by increasing the concessionality of their lending, including via a significant extension of debt maturities. They also committed to do more in the future to help bring debt substantially below 110 percent of GDP by 2022, provided Greece further advances in its fiscal adjustment. Staff assesses the support package sufficient to make the macro-policy framework attainable and the debt trajectory sustainable, although margins remain very limited. The commitment of euro area member states to further conditional relief is crucial to the staff's determination of sustainability. The Section on Debt Sustainability and Financing below discusses the commitments made in more detail.

\section{DISCUSSIONS}

\section{A. The Macroeconomic Framework}

15. The initial review discussions suggested a significantly deteriorated macroeconomic outlook. The problem related to Greece's burgeoning debt overhang. In particular, the political crisis and falling confidence were contributing to a deepening recession which pointed to a need for a more extended adjustment timeline. Medium-term projections showed much higher debt levels, in the mid-150 percent of GDP range in 2020. Such an overhang of debt can lower growth through several channels, including by raising private borrowing spreads, dampening confidence, and compressing domestic demand (see Box 3). In the Greek instance, the interaction of the overhang with very high amortizations of debt looming in the next decade suggested that all of these channels could have been expected to operate.

\section{The package of agreed debt relief measures is expected to help mitigate the acute} macro impacts from a very high debt overhang, but is not enough to offset fully the impact of recent developments (Table 9, Figures 8 and 9). The full package for the review is meant to generate a turning point and unleash a virtuous circle. The macroeconomic framework assumes that 
over the next 12 months Greece is able to make a decisive break with recent political instability and also improve program implementation, while Europe meets its commitment to support Greece. The projected dynamics reflect continued near-term weakness, but capture this eventual reduction of uncertainty and restoration of confidence:

- The recession is expected to be deeper in the near term (Figure 10). GDP is projected to decline by 6 percent in 2012 (compared to a decline of 43/4 percent under the program) and by a further $4 \frac{1}{4}$ percent in 2013 (compared to stabilization). Although euro area politicians now appear to have pulled behind keeping Greece in the euro, implementation risks will remain elevated in Greece for some time, until the political situation becomes less fragile. Slowly receding uncertainty, together with tighter liquidity and faster wage adjustment, is projected to suppress domestic demand, even while worse external conditions are projected to slow export growth. In this environment, fiscal adjustment is also projected to have a larger impact than before (and fiscal multipliers were increased to around one). ${ }^{2}$ However, the

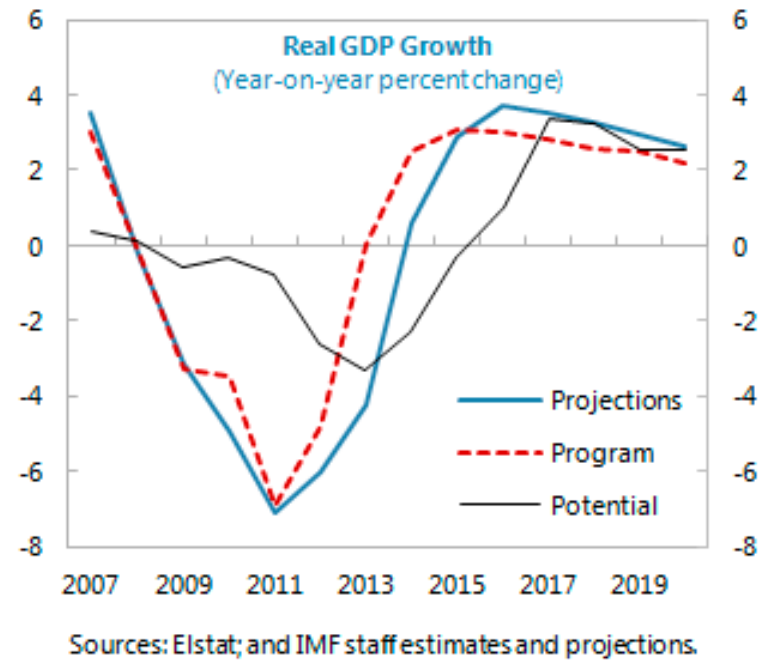
extension of the fiscal adjustment path will help to partially cushion against these effects (without the extension, projected output could be at least 11/2 percent lower in 2013). confidence, further fiscal adjustment in 2015-16 is expected to be a significant drag on the recovery. Meanwhile, potential output will be affected by delayed structural reforms, lower investment, emigration, and loss of human capital (although lower wages are expected to lead to an increase in the labor intensity of production, and thus bring down unemployment). Thus, cumulative output growth between 2012 and 2020-when Greece is expected to return to full employment-is now projected to be 5 percent less than projected at program approval. Growth projections beyond 2020 have been kept close to original program projections (and reflect a shrinking population and receding productivity gains from structural reforms).

\footnotetext{
${ }^{2}$ Estimates from recent work covering various country adjustment episodes show a wide potential range for fiscal multipliers, rising to as high as 1.75 (see October 2012 WEO). Such ex-post estimates must be interpreted with caution, since they partly control for various factors that are unrelated to fiscal developments; for instance, in Greece the political crisis and rising euro exits fears and the impact that these had on confidence.
} 
- External adjustment is expected to proceed at a faster pace (Table 10). Cost and price competitiveness gains should exceed previous expectations. Higher unemployment, stronger effects of the labor market reform, and cuts in the public sector are projected to reduce wages and prices relative to the original program through 2020. Meanwhile the current account will benefit from the recently agreed debt relief deal for Greece (to an amount of about 2 percent of GDP per year on cash basis), and from steeper import compression (as domestic demand contracts faster, and as faster gains in competitiveness encourage import substitution). Overall, the current account is now expected to achieve balance on an accrual basis by 2015 .
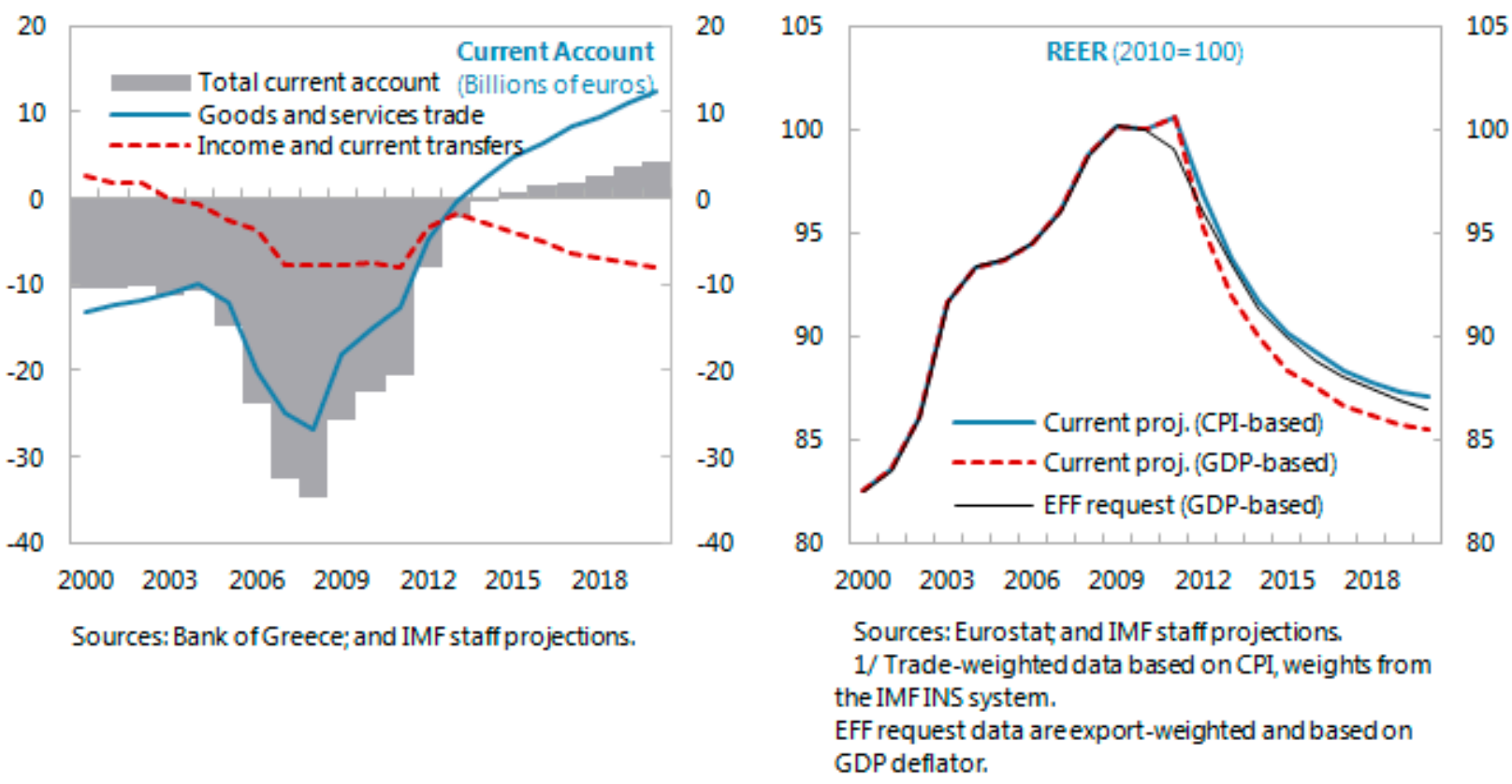

Banking system credit and deposits are expected to resume growth in 2014, in line with economic recovery (Table 11). Although projected deposits are more or less in line with the projections at the time of the arrangement request, private credit growth projections have been revised down somewhat to reflect a weaker economy (which reduces credit demand and hurts bank profitability), and because banks need to make room for higher treasury bill holdings (to help finance the government). Deposit and credit growth are expected to resume in 2014, as under the original EFF-supported program. Reduction in reliance on central bank financing will occur more slowly, given banks' tighter liquidity conditions.

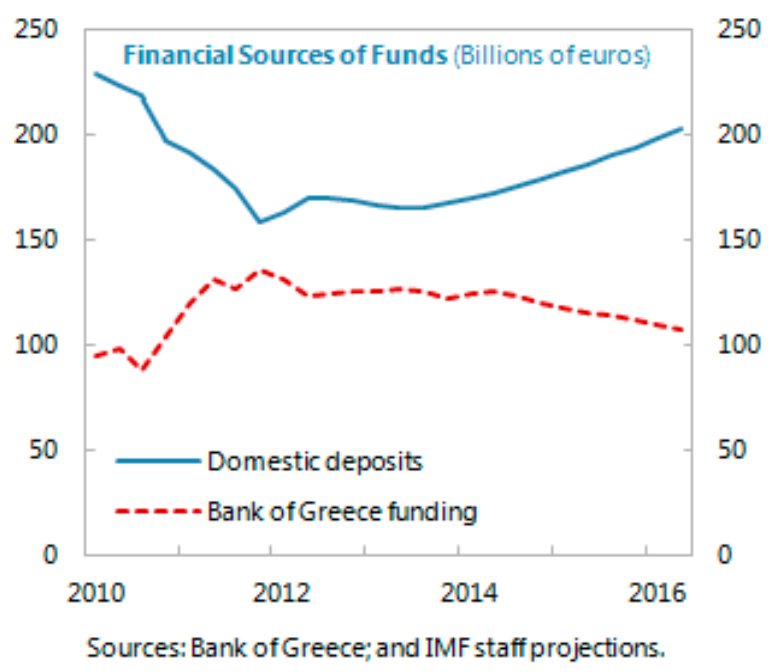


17. Risks to the outlook remain significant. The economy remains extremely vulnerable to confidence shocks, in light of implementation risks and political fragilities. Tight financial conditions are a significant risk, given heavier reliance on Treasury bills in lieu of higher euro area financing (this could involve more crowding out of private sector loans than anticipated). On the external side, growth in trading partner countries could be more sluggish, and this, combined with tighter liquidity conditions, could also increase the size of fiscal multipliers going forward. On the positive side, the rapid reduction in labor costs may facilitate more adjustment through prices rather than quantities, while conclusion of upcoming reviews on schedule could spark an earlier and stronger revival of confidence.

\section{B. Economic Policies}

\section{Structural reforms}

\section{The authorities and EC/ECB/IMF staff agreed that laying the basis for recovery and} growth was the most urgent priority for Greece. The new government recognized that structural reforms to date had been uneven at best, and that a reinvigoration of the reform agenda would be critical to boost potential growth. They also acknowledged the management challenges in this area, and the resistance of vested interests, including the bureaucracy. Progress was made in restarting reforms covering product and service market liberalization, the business environment, and the labor market, all areas where significant rigidities linger. However, implementation timelines will remain extended in some areas (Table 12).

19. Significant steps were agreed to liberalize product and service markets (MEFP १8). Wage adjustments need to be translated into price adjustments to help promote exports (and import substitution), and also to help protect real wage incomes.

- The work on regulated professions remains central. Regulated professions-which number over 500 in Greece and account for about onethird of employment-are subject to tighter restrictions than the OECD average. ${ }^{3}$ As agreed at the outset of the EFF-supported program, the discussions took a sector-by-sector approach. The focus was on eliminating key specific restrictions, for instance on entry, minimum prices, and the mandatory use of services. The professions targeted in this round included several that are important for lowering consumer costs (real estate brokers, private schools, primary care services),

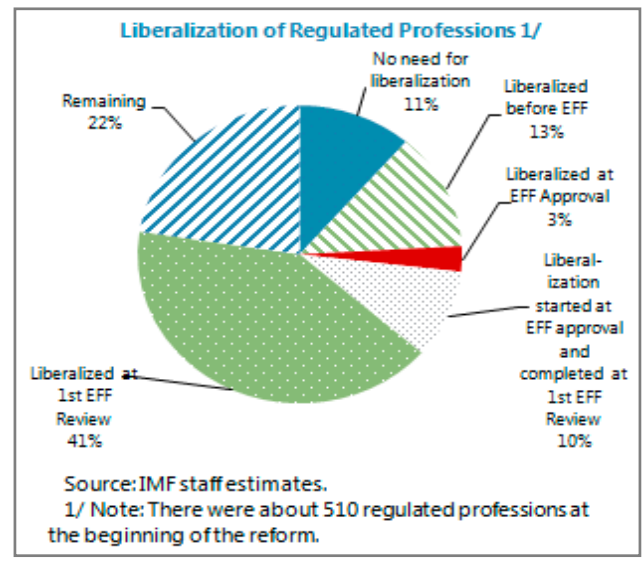

\footnotetext{
${ }^{3}$ See OECD, 2011, "Economic Policy Reforms 2011: Going for Growth."
} 
reducing business transaction costs (lawyers, custom brokers), and reducing business input costs (stevedores, accountants). Some of the liberalization built on measures already undertaken in some professions (lawyers and accountants) (MEFP, Annex I.2). Including the steps taken in this review, the work to date has so far managed to eliminate, de jure, excessive restrictions on most professions, although it will likely yet take some time for de facto conditions to change.

- The discussions also focused on product markets, where the authorities' work has lagged significantly. The review focused mainly on opening up the retail sector to greater competition, in particular by removing restrictions that inhibit scale economies (smaller stores account for most grocery sales in Greece, compared to the EU average of 20 percent of sales, leaving Greece lagging EU-15 average productivity levels by $30-40$ percent). ${ }^{4}$ The discussions also identified changes in the fuel market and in transportation services designed to increase competition in the retail fuel market and increase the range of transportation services in the tourism industry (MEFP, Annex I.1).

- Important steps still lie ahead, and the discussions established some priorities. The target to complete the liberalization of regulated professions by end-2012 was retained, which would entail removal of excessive restrictions for the remaining 20 percent of professions. This will be followed by work, during the first quarter, to liberalize a host of transportation services (airport, seaport, intercity bus, and domestic ferry services). Such reforms are aimed at strengthening the competitiveness of Greece's crucial tourism industry, including by reducing high airport charges relative to regional competitors. Looking further ahead, the authorities aim to screen the construction materials, food processing, and tourism sectors for restrictions, since these sectors are seen as having great potential to contribute to Greece's recovery.

\section{The authorities agreed to renew their effort to tackle the still-massive problems with} Greece's business environment (MEFP 19). With investment expected to be a key component of Greece's recovery, it is imperative that barriers and rigidities be removed. However, the review was only able to make progress in resetting the timeline and establishing next steps:

- $\quad$ The authorities will renew their work towards implementing long-delayed licensing reforms and building up recently introduced trade facilitation reforms. The full implementation of the licensing framework for manufacturing and projects with environmental impact has been rescheduled for Q1 2013. Trade facilitation reforms, including the establishment of a single integrated information system and a one stop shop for export facilitation, are targeted for

\footnotetext{
${ }^{4}$ See McKinsey\&Company, 2012, "Greece 10 Years Ahead."
} 
2013-15. As noted in the fourth review of the previous SBA, these should help reduce the high time and administrative costs for exporting companies in Greece. ${ }^{5}$

- Judicial reform measures have had to be postponed again. These reforms continue to focus on reducing the case backlog; streamlining the Code of Civil Procedure; and publishing court data to strengthen court performance and accountability. In the area of case backlogs, the authorities have reset the deadlines to be more in line with their capacity. In Greece, progress in tackling the backlog problem has been very limited due to judicial independence challenges (as the backlog problem lies in the court process itself) and the lack of political commitment.

- The review defined next steps for the upcoming 12 months. The focus will first be on eliminating barriers to competition. The authorities will conduct an in-depth assessment of 13 key sectors, including agriculture and energy, with the objective to reduce by one quarter the cost of red tape (which is estimated by the OECD to be about 7 percent of GDP a year, almost double the EU average). The authorities will use the OECD toolkit, which helps to identify competition barriers and develop less restrictive measures to achieve policy objectives. A second focus will be on eliminating nuisance taxes, which have proliferated in Greece (including financing entities outside the general government). International experience suggests that an excess of small levies significantly increases business transaction costs, especially for small firms, impairing GDP growth. ${ }^{6}$

\section{The authorities have taken some further steps to further reform the labor market} (MEFP आ10). With labor reforms to date having delivered a bigger-than-expected impact, the authorities were reluctant to take deep upfront actions, as envisaged under the program, but agreed to a phased approach:

- A timetable has been established for a new minimum wage system, although it requires one issue to be revisited in a

year. The authorities have, as a prior action, shifted the power to set minimum wages to the government, thus meeting a commitment in the program. This should help address the significant insideroutsider problem that has characterized Greece's labor market (the minimum wage was previously set by a nonrepresentative group of employers

\footnotetext{
${ }^{5}$ See IMF Country Report No. 11/68.

${ }^{6}$ PricewaterhouseCoopers and World Bank, 2012, "Paying Taxes 2013."
}

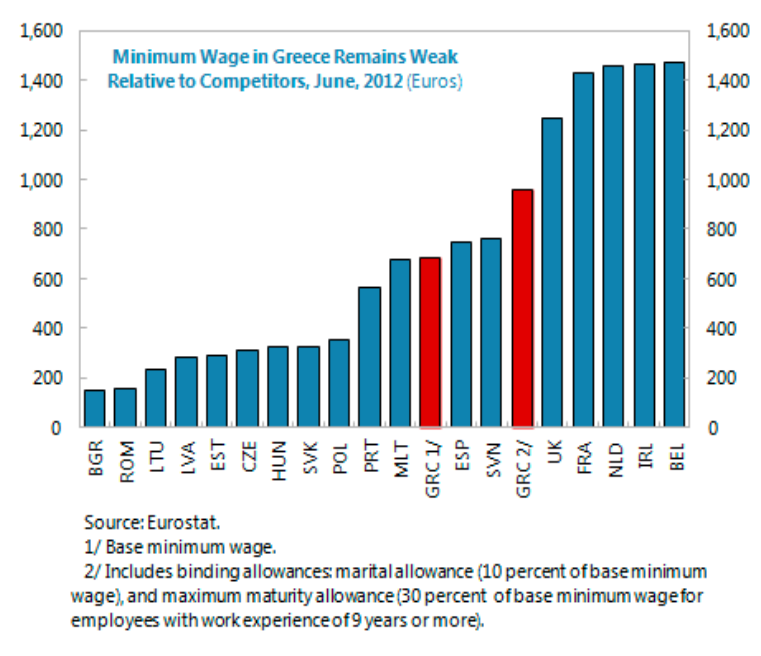


and unions). However, the authorities have not yet fully moved to a single minimum wage, per their program commitment. In particular, they have not yet removed maturity allowances, which were grandfathered for existing employees. This decision will be reviewed in early 2014, informed by developments in the labor market, and administrative feasibility.

The authorities were not technically prepared to address Greece's high tax wedge, but alternative upfront measures were identified to reduce nonwage costs. The plans to reduce the tax wedge had to be rephased to $2013-15$, as the authorities had not been able to fully evaluate options to finance rate reductions (e.g., adjustments to broaden the tax base). To achieve the needed upfront reductions in unit labor costs through this channel, and thereby mitigate pressures for nominal wage declines, the focus shifted to other nonwage costs, including severance pay

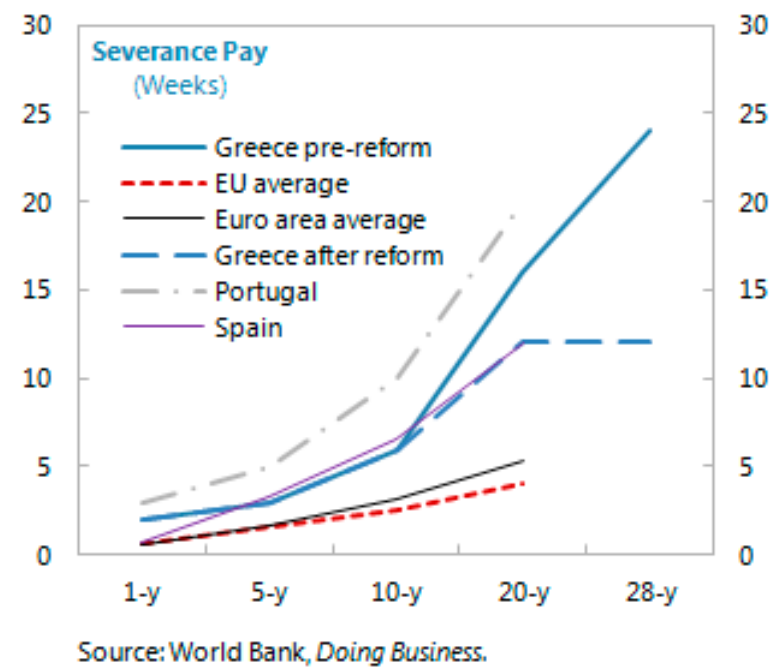
and administrative burdens. Regarding severance pay, the authorities agreed to reform the system to reduce both the notification period (from 6 to 4 months) and the maximum severance (from 24 to 12 months), allowing for some grandfathering. The reform leaves in place a still generous level of severance compared to EU average, recognizing that Greece's unemployment benefits are less generous than elsewhere. Concerning administrative burdens, the authorities agreed to eliminate various pre-approvals by the labor inspectorate (e.g., for work schedules and overtime). These are unnecessary for control purposes and are considered one reason why informal labor arrangements arise.

\section{Privatization}

22. The government committed to restart the stalled privatization process (MEFP $\llbracket 12$ ). The authorities recognized that this would also be crucial for the growth agenda and was not just a matter of generating financing. Privatization is indeed a key element towards a decisive break with Greece's previous failed model of public sector led growth. Discussions focused on removing obstacles to accelerate sales, securing the process against political interference, defining steps to enhance the accountability and transparency of the privatization fund, and reassessing the scope and timeline for asset sales.

\section{The authorities have made further progress in specifying assets for privatization, although much work still lies ahead concerning real estate assets (MEFP ף13). The list of} companies and concessions remains unchanged, but value estimates have been updated based on pre-tenders and profitability analysis by financial advisors. For real estate assets, the authorities have 
identified a pool of 3,150 properties with an estimated market value of $€ 10$ billion. This will provide a basis for sales over the next 2-3 years, and from this group the authorities will transfer 1,000 fully identified assets to the HRADF in 2013. Beyond the pool of 3,150 assets, the authorities have identified some 10,000 additional real estate assets, with an estimated value of $€ 8$ billion, which could form the basis for later privatizations.

\section{Steps are being taken to move the process forward, although significant cash results are still some way off (Table 13, MEFP $\llbracket 14-15)$ :}

- The ownership of assets is being transferred to the HRADF. Ownership by the HRADF is important to signal a political decision by the government to divest the asset and allows the HRADF to lead the divestment process. All assets ready for sale are held by the HRADF in off-balance sheet accounts to maximize flexibility to restructure them (loss-making enterprises or assets that require cash injections for further restructuring remain with the government since the HRADF has no means to finance their deficits).

- Legal impediments are being removed. The impediments fall into several categories, including (i) the presence of golden shares and/or restrictions on how much of some assets can be sold to private investors; (ii) company statutory provisions that deviate from private law; (iii) state aid issues to be resolved with the EC; (iv) unfinished regulatory frameworks for previous monopolies; ( $v$ ) the need for restructuring laws, (vi) the need to establish/extend licenses in some cases (e.g., the water companies); and (vii) for real estate, missing zoning and land planning permits. Through November, the authorities had managed to largely address the first two of these categories, and have also made progress in state aid clearance.

- $\quad$ Tenders are being accelerated, but significant sales proceeds are still some way off. The HRADF has brought three projects to the tender stage over the last 12 months. With legal impediments removed, the objective is to have 21 objects tendered by June 2013 (compared to only seven since the setup of the HRADF). With adherence to this schedule, sales proceeds could begin to accrue in earnest during H2 2013.

\section{Strong political resistance to privatization forced the review of the issue of HRADF} independence, transparency and accountability (MEFP आ16). The recent amendment to the privatization law mandating the HRADF to take account of recommendations of Parliament's Economic Committee threatens to introduce new barriers and delays into the process. Given the crucial role of privatization, it was agreed that in the event of any delays-measured both by reference to the amount of objects privatized and proceeds realized-steps would be taken to strengthen the HRADF, including eliminating this ex-ante review by parliament and changing the Board. At the same time, it was recognized that the transparency and accountability of the HRADF would need to be strengthened: while it should not be micro-managed by parliament, it does need to be accountable for its actions. Several steps were agreed, including publishing plans and HRADF financial accounts. 


\section{Privatization proceeds projections were adjusted down in light of market conditions} and already incurred delays (MEFP आ17). Heightened implementation risks and lingering euro exit fears are expected to depress demand for privatized assets, weighing on sales volumes and prices; an updated bottom-up assessment suggests that fewer real estate assets are good prospects for sales; and more evident political constraints and regulatory and judicial barriers suggest that the process will take more time. Thus, cumulative proceeds are now set at $€ 6$ billion by 2014 , $€ 10$ billion by 2016 , and $€ 23.5$ billion through 2020 (including the $€ 1.6$ billion realized to date). This is about half of what was projected at the time of the approval of the Extended Arrangement. The relatively backloaded profile for receipts reflects the fact that it will take time to sell bank equity acquired during recap (about half the expected proceeds), and time to prepare real estate assets for sale.

\section{Financial sector policies}

27. Discussions focused on the next steps towards financial sector reform (MEFP $\llbracket 18$ ). The new government recognized this to be crucial to their aim to stabilize the economy and promote recovery. Agreement was reached on refinements to the recapitalization and resolution process, the process for operational restructuring of banks, steps to strengthen governance at banks and the HFSF, and steps to adapt the supervisory framework to the changed environment.

\section{The framework for recapitalizing banks has been preserved, albeit with a few} refinements relative to the original EFF-supported program (MEFP आ19): end-September 2012 to end-April 2013. The longer period is needed to stagger the rights issuance of each of the core banks, in order to avoid saturating the very thin market for Greek bank equity. Preparatory steps have been taken as a prior action for the review, including communication to the banks of their final capital needs.

Conditions for continued private management of core banks have been tightened. The program retains the objective of keeping core banks run by private shareholders, subject to their injection of 10 percent of the new equity capital (counting any increase in capital from acquisitions). To address concerns about governance, specifically the fitness and propriety of managers and owners, an enhanced due diligence of the banks' related party lending activities was required (below).

The framework for non-core banks was finalized. Noncore banks are likely to face significant challenges to their viability due to limited business opportunities and higher funding cost. For this reason, the program does not anticipate recapitalizing them alongside private shareholders. To operate on a stand-alone basis, these institutions will need to be fully capitalized with private resources, or merge with other sound institutions on the basis of a viable business plan before end-April 2013. In the interim, these banks will remain under enhanced supervision. 
Steps and Timeline for the Recapitalization Process

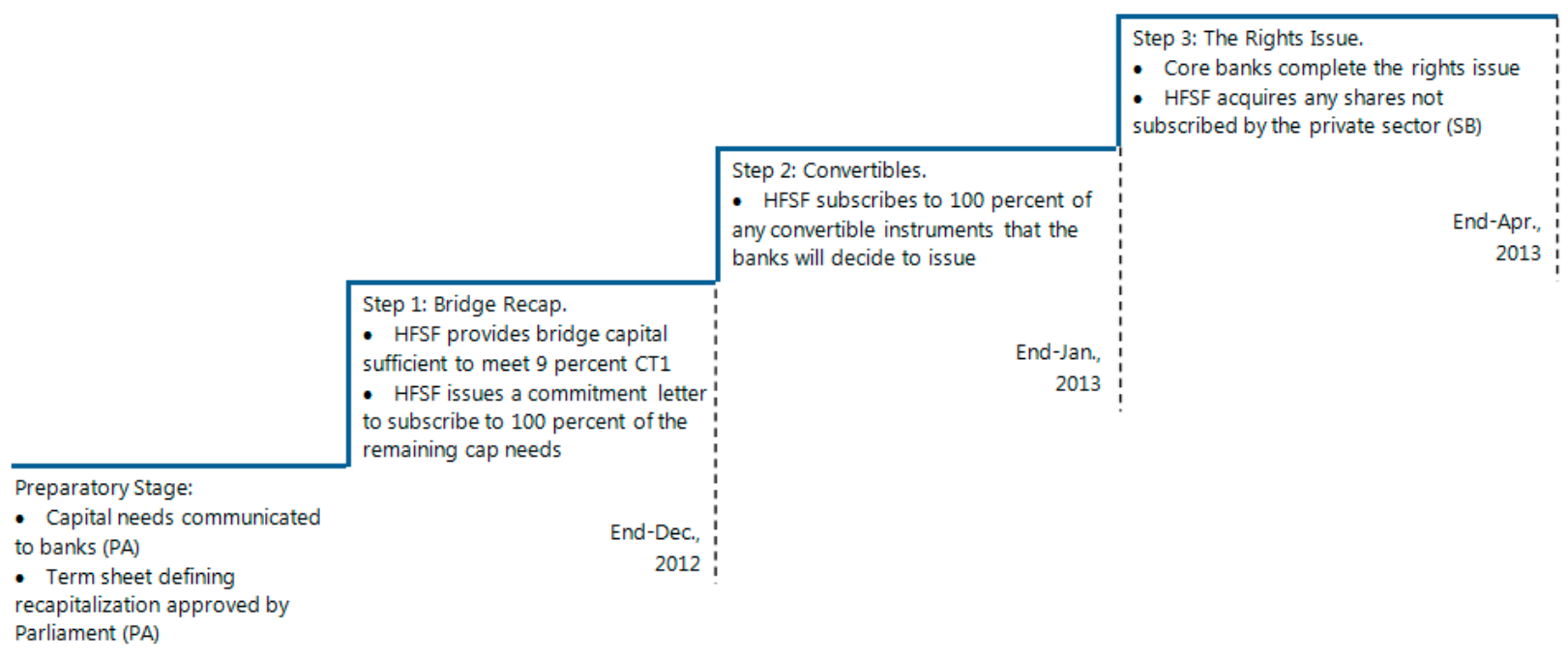

29. The framework for bank resolution was further refined (MEFP $\llbracket 20-21$ ):

The timeline was extended to end-June 2013. The most likely scenario involves smaller banks being acquired by core banks in purchase and assumption transactions. Thus some time is needed after core banks are recapitalized (at end-April) to allow potential acquiring banks to perform due-diligence and evaluate a potential P\&A transaction. With the resolution of ATE and Hellenic Postbank out of the way very soon, the authorities can perform their due diligence and determine the carve-out perimeters for the good assets ahead of time, to facilitate the process for potential acquirers.

- To minimize resolution costs, the authorities will develop an approach for the management of assets left in bad banks. Based on international best practices, options include: (i) an enhanced role for the liquidator with stronger powers to manage assets along business lines or (ii) a more centralized approach that would involve pooling bad assets to facilitate economies of scale and to ensure a consistent approach. The authorities agreed to seek foreign expert assistance to choose a model best suited for Greece.

- The BoG will set out a strategy to deal with cooperative banks (MEFP I21). While the sector is not systemically important, its deposits are in practice fully protected by the state (the sector comprises 13 institutions and holds deposits of about $€ 2.6$ billion). Restructuring options include: (i) integrating these institutions into the viable banks; or (ii) merging them into a core cooperative unit to be recapitalized with a viable business plan. 


\section{Against the backdrop of the revised recapitalization and resolution framework, the} authorities have decided on how they intend to handle specific banks. The authorities will resolve Hellenic Postbank no later than end-January 2013, with

Postbank's good bank to be acquired via an open-bid process. The authorities will allow the NBG-Eurobank merger to proceed. This could have significant business and funding advantages, but staff cautioned against excessive concentration in the system (if the merger is completed, the new NBG would control around 40 percent of system deposits). It is expected that the EC's DGCompetition will review the anti-trust Estimated Structure of the Banking Sector, 2013Q1 aspects of the deal.

\begin{tabular}{lrrr}
\hline & $\begin{array}{c}\text { Deposits (billions } \\
\text { of euros) }\end{array}$ & $\begin{array}{c}\text { Deposit share } \\
\text { (percent) }\end{array}$ & $\begin{array}{c}\text { Assset share (risk- } \\
\text { weighted, } \\
\text { percent) }\end{array}$ \\
\hline NBG/Eurobank 1/ & 58.6 & 40.2 & 41.4 \\
Alpha 2/ & 32.4 & 22.3 & 27.4 \\
Piraeus 3/ & 32.1 & 22.1 & 21.2 \\
Postbank 4/ & 10.9 & 7.5 & 3.1 \\
Other & 11.5 & 7.9 & 6.8 \\
\hline
\end{tabular}

Sources: Bank of Greece; and IMF staff estimates.

$1 /$ Assuming the planned merger is completed by end-2013Q1.

2/ Includes assets and deposits of recent acquisition of Emporiki.

3/ Includes assets and deposits of recent acquisitions of ATE and Geniki. 4/ TT Postal bank will be resolved.

\section{The authorities and staff reached understandings on further steps to strengthen the banking system, post-recapitalization (MEFP $\llbracket 23$ ):}

- Banks receiving state aid will need to produce business plans confirming their medium-term viability. Ensuring the successful implementation of these plans is critical, given that the banks' recapitalization plans rely heavily on internal capital generation through net profits before provisioning. Furthermore, these business plans outline how the banks will gradually extricate themselves from reliance on central bank funding over the next three years. The HFSF and the monitoring trustees (see discussion below) will check banks' adherence to their restructuring plans and report on a semi-annual basis to the EC/ECB on progress.

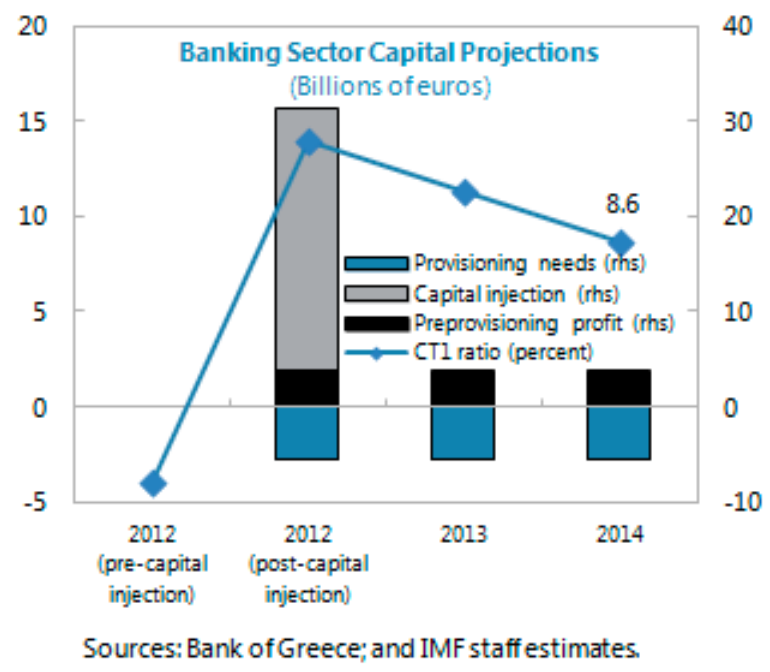

- NPL resolution and debt workouts will be enhanced. It was agreed that banks should put in place appropriate resolution frameworks to deal with problem borrowers, including placing nonperforming assets in a dedicated unit (under a separate management team) that would specialize in facilitating debt workouts (an approach that some European banks are currently using). The use of asset management companies has not been ruled out. However, the discussions so far point to challenges associated with the use of such vehicles, including 
the lack of funding to support the operation, and a lack of high quality instruments to replace the transferred assets in the banks' balance sheet.

The legal framework to restructure viable debtors will be revised, in line with the authorities' commitments under the program. The current framework does not provide sufficient incentives or penalties to encourage debtors to engage in loan restructuring negotiations. While many proposals have been put forward by Greek parliamentarians, they have lacked an appropriate balance between the interests of creditors and debtors, thus presenting fiscal risks, threats to bank solvency, and risks to the payment culture. This work was rephased from Q2 2012 to Q1 2013, to allow further assistance from international experts.

32. The authorities specified additional safeguards to ensure appropriate oversight and governance of banks (MEFP 124). Either state control or banks remaining in private control (with a minority of the equity base) raise potential concerns. The program aims to mitigate concerns through several measures:

- A review of the fitness and propriety of bank management and owners. To this end, the HFSF-hired auditing firms to investigate whether loans to related parties are within legal limits, are properly monitored to avoid misreporting to the BoG, and are not subject to special lending terms or loan restructuring facilities. Its findings were communicated to the BoG. In general, auditors did not report specific misconduct in banks, but information on banks' internal procedures will be subject to further analysis by the BoG.

- The placement of monitoring trustees in banks. International accounting firms will be appointed as trustees, and their terms of reference will include the participation of overseasbased partners and managers. The trustees will verify proper governance and the use of commercial criteria in key policy decisions, including follow-up on findings regarding related party lending. They will be responsible for overseeing the implementation of restructuring plans, and will report on progress to the HFSF and the EC/ECB. The role envisioned for these trustees goes beyond usual practice. This approach was deemed appropriate given the large amounts of public money injected into banks and the risks associated with the rights given to minority shareholders.

- Enhanced HFSF transparency. The EC and ECB observers at the HFSF will be given increased access to information ahead of any Board discussion. They will also liaise closely with the monitoring trustees. This will allow Europe to conduct more timely oversight. In addition, the HFSF will publish more details regarding its operations on a semi-annual basis.

Strengthened bank supervision (MEFP 125). The BoG will revise its supervisory model to increase its monitoring of key banks ratios and intensify onsite inspections. In support of this, banks will be asked to standardized their asset quality disclosure (current accounting practices and reporting in Greece are not harmonized across banks in various areas, 
especially relating to NPLs). This should, inter alia, allow the BoG to follow more closely the implementation of the banks' business plans, and ensure that core institutions generate internal capital and increase loan provisioning as envisaged in the recapitalization program. To provide incentives to the BoG to further strengthen supervision, the authorities agreed to undertake a new assessment of compliance with the Basel Core Principles (BCPs) in 2014 (the last BCP assessment was completed in October 2006, but the banking environment in Greece has since changed, and the BCPs have been refined).

\section{Funds earmarked for bank recapitalization remain sufficient. The revised}

macroeconomic framework for the program implies a higher peak for nonperforming loans and lower asset valuations. These factors would tend to raise capital needs for recap and resolution.

Additional needs have also been identified in cooperative banks. However, a revision of CT1 targets from 10 to 9 percent (to align them with European Banking Authority recommendations on capital buffers), and capital injections by the owners of some banks have helped offset this impact. Overall, the envelope of about $€ 50$ billion was found adequate with sufficient buffers to deal with stress scenarios and to address potential needs in banks' foreign subsidiaries. The Bank of Greece's analysis indicates that banks could sustain a peak NPL level of 40 percent, assuming a coverage ratio of 50 percent (this is in line with peak NPLs in past sovereign debt crises, such as Argentina (20 percent), Ecuador (40 percent) and Uruguay (36 percent)). In addition, the core banks hold tax credits for about $€ 3.2$ billion associated with PSI losses that have not yet been incorporated into program capital estimates. While buffers appear sufficient for now under the present macro framework, an additional top-down verification of capital needs will be conducted by end-December 2013. This stress test will assess whether the banking sector will remain well capitalized through 2014, under updated macro assumptions and given performance through June 2013.

\section{Updated Capital Needs of the Banking Sector, 2012}

\begin{tabular}{lr}
\hline Banking sector envelope, EFF & $\mathbf{5 0 . 0}$ \\
Factors increasing the envelope & $\mathbf{2 . 4}$ \\
Higher recap. costs for the core banks & 0.6 \\
Resolution of cooperatives & 1.3 \\
Higher ATE resolution costs & 0.5 \\
Factors decreasing the envelope & $\mathbf{3 . 0}$ \\
Lower resolution cost for banks other than ATE & 1.0 \\
Capital from acquisition of foreign subs & 0.7 \\
Conversion of hybrids into equity & 0.6 \\
Reduction of CT1 target to 9 percent & 0.7 \\
Debt buy-back 1/ & 0.0 \\
Banking sector envelope, Dec. 2012 & $\mathbf{4 9 . 4}$ \\
Memorandum item: & \\
Deferred tax credit 2/ & $0-4.4$ \\
\hline
\end{tabular}

$1 /$ The effect of debt buy-back is estimated to be neutral as the loss of income to the banks is expected to be offset by a purchase price slightly higher than the book value

2/ This item is under consideration by the authorities and as such cannot count towards buffers in the envelope yet.

\section{Fiscal policy}

\section{The review focused on recalibrating the fiscal adjustment timeline and fully defining}

the adjustment strategy. The government acknowledged a need to adjust quickly to help address concerns about Greek solvency, but also agreed that the pace of adjustment had to reflect macro considerations. The discussions revolved around ensuring a credible, efficient and well-targeted 
package of measures that would protect the most vulnerable, and distribute the adjustment burden equitably (to enhance its political sustainability).

\section{The authorities and their European partners agreed to extend the timeline for fiscal adjustment, allowing a recalibration of required fiscal measures (Table 14, MEFP \27-28):} A more feasible adjustment path. The new path reaches the targeted primary surplus of $4 \frac{1}{2}$ percent of GDP two years later, in 2016. The benefit of the expanded timeline is immediate: in 2013 the targeted adjustment in the primary balance drops from 3 percent to $1 \frac{1}{2}$ percent of GDP ( $41 / 2$ to 3 percent of GDP, when measured by the structural primary balance). The end-point target of a $4 \frac{1}{2}$ percent primary surplus remains ambitious, but it is in line with projected structural positions in other European countries. Greece has also achieved and maintained this level for a few years before euro accession.
E.U.: Large Primary Surpluses

(Longest periods of average primary surplus above 4.0 percent of GDP)

\begin{tabular}{lr}
\hline Belgium & $1987-2008$ \\
Denmark & $2003-08$ \\
Finland & $1998-2004$ \\
Greece & $1994-00$ \\
Hungary & $1995-2001$ \\
Ireland & $1988-2004$ \\
Italy & $1994-2001$ \\
\hline
\end{tabular}

Source: IMF staff estimates.

- $\quad$ Fewer required measures. The longer timeline reduced the amount of fiscal measures necessary to complete the fiscal adjustment. Had the original program targets been maintained, Greece would have had to implement 11/1/2 percent of GDP in measures in 201314. Moreover, the country would have been forced to over-adjust, given the still-large projected output gap in 2014. Extending the timeline helps Greece benefit from a mild expected cyclical recovery of revenues, reducing the total needed measures to $73 / 4-83 / 4$ percent of GDP ( $€ 131 / 2$ billion through 2014 , and a further $€ 2-4$ billion in 2015-16, depending on the strength of the cyclical recovery). Moreover, from a macro perspective the amount of gross new measures overstates the impact, since some 21/4 percent of GDP of the new measures is just required to offset expected declines in the yields from past measures.

36. The authorities' adjustment strategy focuses on reducing expenditures (MEFP 130 ). The authorities recognized that they needed to refocus on spending cuts, after relying heavily on tax rate increases during 2010-12. Given the size of needed cutbacks, they also recognized that cuts would inevitably fall on pensions, wages, and social benefits, where spending exploded after Greece's euro accession and which together constitute almost three-quarters of spending. To make this more acceptable to the public and to reassure that the adjustment burden would be more fairly distributed, the government decided on a two-pronged approach: (i) carefully targeting the cuts to protect the most vulnerable; and (ii) matching the cuts with immediate efforts to reduce waste in government operations and to improve tax compliance. However, the authorities recognized that the reforms to address waste and tax fraud-which involved complex and time-consuming administrative changes-would only make a modest contribution to fiscal adjustment in the near term. 


\section{Against this backdrop, the authorities have defined and approved a very ambitious}

package of reforms. The authorities have identified measures amounting to 5 percent of GDP in 2013 and an additional 21/4 percent of GDP in 2014. They have specified a total of about 80 measures cutting across every category of spending and taxation, with the package approved as a prior action for the review (Table 15, MEFP, and Annex V). Importantly, the bulk of the measures have been implemented upfront as prior actionssome three-quarters of the package-and are parametric reforms that require minimal further implementation action (that is, the wage, pension, and social benefit measures). The package resembles the pattern of large consolidation plans currently implemented in other European countries, but with a greater share of savings from wages and social spending in the total spending cuts (around 80 percent vs. 60 percent), and a greater contribution of direct taxes to the total revenue increase (54 percent vs. 46 percent) compared to other countries with large adjustments.

\section{The package brings Greece's public finances closer to European fiscal trends. Total} primary spending is expected to fall to 41 percent of GDP by 2014 (Figure 11), while revenues are expected to remain around 43 percent of GDP (compared to projected euro area averages of 45 and 46 percent, respectively). On the spending side of the budget, cuts in special wage regimes, the elimination of various bonuses and planned cuts in public employment will bring the ratio of wages to GDP closer to the European average of about 101/2 percent. The pension measures, which bring forward the impact of the 2010 reform, will reduce pension spending from 17 percent to about 14 percent of GDP in 2013, still above, but much closer to the euro area average of 12 percent. On the revenue side, tax reform is expected to keep direct tax revenues close to 10 percent of GDP (still 2 percentage points behind the euro area average, reflecting conservative assumptions about tax administration gains).

\section{The spending and tax reforms specified should help to preserve social protection and strengthen tax system simplicity (MEFP $\llbracket 30-31$ ):}

- The package has been designed to protect the most vulnerable (MEFP आ31). On the spending side, cuts in both public wages and in pensions are progressive in nature (for pensions, those below $€ 1000$ per month have been exempted, while the cuts increase to 20 percent for those exceeding $€ 3000$ per month). On the revenue side, the income tax 
reform raises effective tax free income to $€ 10,000$ - from the current $€ 5,000$-through an overall tax credit that transfers a greater share of the tax burden toward higher incomes. Finally, the authorities have expanded unemployment insurance benefits (currently on a pilot basis). They are also in discussions with the EC to leverage available structural funds to expand their job training programs.

The tax reforms will not only deliver substantial revenue, but also represent a major simplification of the tax system (Table 16). For the PIT the number of tax brackets has been reduced from 8 to 3 and allowances, tax credits, and deductions have all been curtailed. The self-employed have been better brought into the tax net. Finally, many types of capital income will now share the same moderate flat rate (20 percent). These changes will reduce taxpayer compliance costs, better distribute the tax burden, and eliminate distortions in capital allocation. Looking forward, the authorities still need to replace the present discretionary and opaque process for granting tax incentives with a more rulesbased approach (e.g., an allowance for corporate equity), and address sources of potential erosion of the corporate tax base (e.g., thin capitalization rules).

\section{There are two areas where work will continue:}

The strategy for fiscal adjustment in 2015-16 has not yet been fully identified (MEFP 132). To close the estimated gap of up to $€ 4$ billion, the authorities intend to focus on retaining expiring measures, base broadening, and gaining efficiency savings from longterm structural reforms. The largest gains would come from extending expiring revenue measures (i.e., the solidarity surcharge on PIT). Staff also sees potential in continuing the reforms of the social benefits system, which remains complicated and inefficient. The fifth review, coinciding with the preparation of the 2014 budget and MTFS update, will aim to define specific measures.

The authorities have not yet finalized plans for staff reductions. They committed to transfer 27,000 staff to a new mobility scheme, a successor to the seldom-used labor reserve. They have initiated this scheme by transferring 2,000 staff as a prior action (still well short of the 15,000 they should have transferred to the labor reserve by end-2012). Targets for dismissals are now needed (the framework would be expected to follow the model laid out for the labor reserve, where the reduced pay received for a one-year period prior to dismissal would count against severance). Targeted dismissals are essential to help the authorities realize their operational savings goals, and will help to ensure the right mix of skills in the future public sector labor force (reliance on attrition alone provides no guarantees). The authorities agreed to set targets at the time of the next review, once staffing plans are known (these are being developed with French TA).

41. Contingency plans have been updated and formalized (MEFP \33). Slippages would, as envisioned at the time of approval of the Extended Arrangement, be corrected through expenditure cuts. At the request of Greece's European partners, the procedure has been formalized. The 
authorities will introduce binding sectoral expenditure ceilings, and new correction mechanisms for state enterprises and local governments (below). Ex-ante buffers within-year buffers will also be set via appropriations that will only be released if targets are met. In case of over-performance deemed sustainable, the proportion devoted to debt reduction has been set at 30 percent, while the authorities can use up to 70 percent of any (permanent) windfall revenue on programs targeting the most vulnerable or to support the recovery (e.g. by easing the labor tax wedge). This shift to stronger fiscal contingency rules can help control risks going forward, but successful implementation will depend critically on strengthening fiscal transparency and the institutions for public financial management.

\section{Fiscal institutional reforms}

42. Discussions focused on restarting the stalled institutional reform process (MEFP $\llbracket 34$ ).

The government recognized that success in this area would be crucial to support their fiscal adjustment strategy. In the revenue administration area, political and bureaucratic resistance to reform motivated a change in strategy. Concerning broader public financial management issues, the focus shifted to elaborating fiscal rules and enhanced monitoring mechanisms, although next steps were defined for the expenditure control agenda.

\section{The authorities agreed to strengthen the framework for revenue administration}

(MEFP 135). To address deep institutional resistance to change and prevent political interference, the management and independence of the administration will be reinforced, and the staff will be made more accountable. By early 2014, the authorities will transform the administration into a semi autonomous agency. Concerning staff accountability, strong criteria for hiring, dismissal, and rotation for auditors and other key managers will be established as well as performance targets. The fourth program review-in Q3 2013-will have a focus on revenue administration, to ensure that the agreed changes are indeed being implemented, including removal of underperforming staff. To help inform this mid-year review and subsequent work, new quantitative targets were set for 2013 (TMU, Table 1).

\section{Alongside measures to strengthen independence, a number of steps are being taken to} strengthen operations (MEFP $\llbracket 35$ and $\llbracket 36$ ). With tax administration afflicted with problems at every stage of the collection process, the agenda remains wide (Table 17). The review focused on three key issues, and next steps in other areas:

- Securing adequate staffing in key functions. The authorities agreed to address the significant shortage of auditors covering large taxpayers, high-wealth individuals and debt collection. They have started with the immediate transfer of 150 staff from local tax offices, and this is to be followed by new hiring and further internal transfers during $\mathrm{H} 12013$. Since there have been significant concerns in Greece about the competence and integrity of existing auditors, those transferred will be subject to audits of their wealth. 
- Reforming accounting requirements. The authorities replaced the Code of Books and Records (CBR) with the Tax Recording of Transactions and Reconciliations Code. The new code involves many simplifications, and places all business taxpayers on an equal footing by requiring them to keep standardized records. Moreover, it reduces the power of local tax office managers to override provisions in the code. While some provisions will only come into effect by end-2013, the change should, overall, reduce opportunities for tax evasion and corruption.

- Defining a plan for improving social security collection. The newly adopted action plan (meeting a program structural benchmark) envisages: (i) strengthening the recovery of arrears; (ii) enforcing filing; (iii) a new framework for settlement and penalty arrangements; and (iv) full integration of collection activities into the tax administration in the long run (beginning with consolidation into the largest social security fund). The plan should help the authorities address continuing shortfalls in social security fund revenue and the accumulation of contribution debt (31/2 percent of GDP at end 2011).

- $\quad$ Next steps. The revenue administration needs to gear up its anti-corruption efforts. To this end, a new code of conduct will be adopted by end 2012; a framework to facilitate the reporting of misconduct, protect whistle blowers and centralize decisions on disciplinary actions in the internal affairs unit will be implemented; and the Internal Affairs Directorate of the MoF will commence annual audits of assets of managers and tax auditors. Actions are also needed to support the income tax reform. Thus, by mid-2013 the income tax law will be further simplified and a new Tax Procedure Code will be implemented to tighten installment schemes, introduce modern audit and debt collection methods, adopt more efficient enforcement methods, and reform the penalty and interest regime. Measures will also be introduced to more effectively utilize third party information (e.g., on bank accounts, utility bills, and insurance contributions) to support presumptive taxation under the new tax regime for the self-employed.

\section{Concerning public financial management, the government agreed to strengthen}

Greece's fiscal framework. In addition to a more formal fiscal contingency framework ( $\$ 41$ above), Greece's European partners insisted that Greece adopt stronger fiscal rules as an upfront priority. Thus the government will set quarterly fiscal targets for line ministries, state enterprises, and local governments, and establish mechanisms for sanctions. From a cross-country perspective, annual and medium-term sectoral expenditure ceilings are used in some member states (Austria, France, Italy, and Portugal) and have performed well. The targets should over time create pressures for more careful and realistic budget planning and execution, but, in the short run they are likely to present very significant budgeting and cash management challenges.

\section{The authorities and staff agreed on the next steps towards strengthening expenditure} controls (Table 18, MEFP आ38). This work has become more challenging, given limited capacity and a diversion of resources necessary to set up the new fiscal framework and fiscal rules in the country. 
The expenditure control work is focused on two complementary reforms, which together are key measures to help staunch the flow of arrears:

- $\quad$ Establishing General Directorates of Financial Services (GDFS). These directorates will become the center of financial management in line ministries, replacing multiple units responsible for financial services and consolidating the control of expenditure commitments. They should be in place by end-2012, but to make them fully effective the authorities will need to provide guidance and support, build capacity in the units, and monitor progress. New targets were set for 2013 to facilitate monitoring (TMU, Table 2).

- $\quad$ Extending the use of commitment registers. As a prior action, the authorities brought the national health service-one of the largest spending units in the government-into e-portal reporting. Looking forward, they aim to integrate almost 720 small general government entities into the system of reporting and use of commitment registers (the structural benchmark targets for mid-2013 have been adjusted to reflect their integration).

47. The strategy for clearing domestic spending arrears was updated (MEFP आ39). As envisioned in the Extended Arrangement, the funds allocated in the program to clear arrears will be made available if the claims have been verified, and if agencies comply with basic financial management reforms and report consistent data from commitment registers. However, given the role that delays in the review has played in tightening liquidity, line ministries will not be required to have run no arrears in order to access funds to clear their outstanding stocks. The timeline for arrears clearance was also revised-to run through end-2013, to account for the larger stock of arrears (€9.4 billion as of end-October 2012). An audit, to be completed by end-2013, will help establish whether the clearance program has been successful, and what if any follow-up actions are needed.

\section{DEBT SUSTAINABILITY AND FINANCING}

48. Given the revised policy framework and deeper recession, and assuming no actions to reduce debt, Greece's debt would be unsustainable. Greece's debt would be considerably higher than in the original EFF-supported program, peaking at close to 200 percent of GDP and only slowly declining to 150-160 percent of GDP in 2020. Such a level would be too high to facilitate a return to the market, particularly given the high required amortization of EFSF and GLF loans, and new Greek government bonds. But even assuming that market access could be temporarily restored, high borrowing costs would place debt on a sharply increasing trajectory. A sudden stop

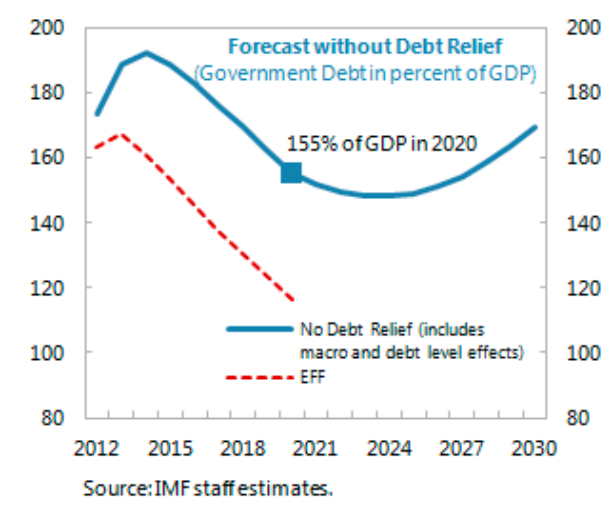
in borrowing would almost certainly occur. 
49. Debt relief was deemed necessary in significant volume, implying a need for European official sector involvement (OSI). Taking into account the potential macro benefits of the relief, the necessary magnitude of measures was estimated to equal about 24 percent of GDP (i.e., to achieve the target of a 120 percent debt-to-GDP ratio by 2020). Given the structure of outstanding debt, it was inevitable that the European official sector would need to play a key role to generate the needed relief.

\section{Greece and its European partners agreed to} a package of upfront debt relief measures and a maturity extension on existing loans (Box 4). The measures adopted include an interest rate reduction on existing GLF loans, and fiscal transfers by member states (of ECB profits related to Greek bond holdings). The Greek authorities also completed, on December

General Government Debt, 2012

(Billions of euros)

\begin{tabular}{lr}
\hline & Proj. \\
\hline General government & 307.2 \\
State & 312.2 \\
Tbills & 18.4 \\
Official sector & 183.6 \\
IMF & 22.3 \\
European Commission & 161.3 \\
Exchanged bonds & 29.9 \\
Non-exchanged bonds & 56.3 \\
Loans & 18.0 \\
Other & 6.0 \\
Subsectors 1/ & -5.0 \\
\hline
\end{tabular}

Source: IMF staff estimates.

$1 /$ Subsector debt is net of intra-government holdings. 18,2012 , a voluntary buyback of bonds from the private sector. Greece's European partners also agreed to a 15-year extension of the maturities on official loans. Together, the latter two measures dramatically reduce rollover requirements post-2020. All told, these measures are projected to deliver some 16 percent of GDP in debt reduction by 2020.

\section{Since this was not enough to restore debt sustainability, Greece's European partners} gave supplementary assurances of additional conditional debt relief. Through the Eurogroup statements on November 29 and December 13, and via separate interactions with the Managing Director, they have made clear that they will: (i) provide 1.4 percent of GDP in debt relief in early 2014 (provided the primary balance target for 2013 is met); and (ii) take measures in 2015 to ensure that debt drops to 124 percent of GDP by 2020 and substantially below 110 percent of GDP by 2022 (provided Greece's primary balance swings to a surplus as programmed in 2014). Time will tell what the full commitment will require, but staff's assessment at this point is that some combination of haircuts on outstanding GLF loans, close to zero interest rates on GLF loans and lower rates on EFSF loans, or long-term transfers will be necessary. 
52. Under the new financing package and provided the program is implemented, debt would be sustainable in the medium term, albeit still with risks (Appendix I).

- Public debt. The debt would decline to 124 percent of GDP in 2020, somewhat higher than the targeted 120 percent of GDP level. Debt dynamics before 2020 are also less favorable, with debt that peaks in 2014 about 10 percent of GDP higher than previously. However, the European commitment to further contingent debt relief point to an eventual recovery of the program debt

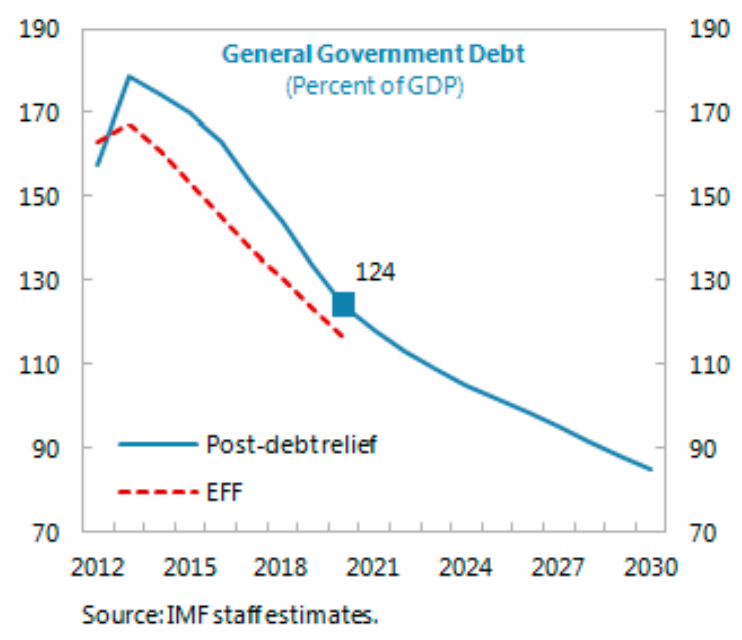
trajectory in 2022 (the original program foresaw debt of 107 percent of GDP in 2022). Nonetheless, risks remain significant. Stress tests single out risks from delays in reforms, more adverse macro dynamics, and non-implementation (leading to euro exit).

- External debt. Net external debt would fall significantly from 109 percent of GDP at end2012 to 59 percent by 2020 according to baseline projections. This is lower than projected at the time of program inception due in large part to the improved path for the current account and the debt buy-back. It remains the case that macroeconomic shocks and policy slippages could result in considerably more adverse debt dynamics.

\section{Taking into full account the maturity} extension on loans, Greece's debt in fact now

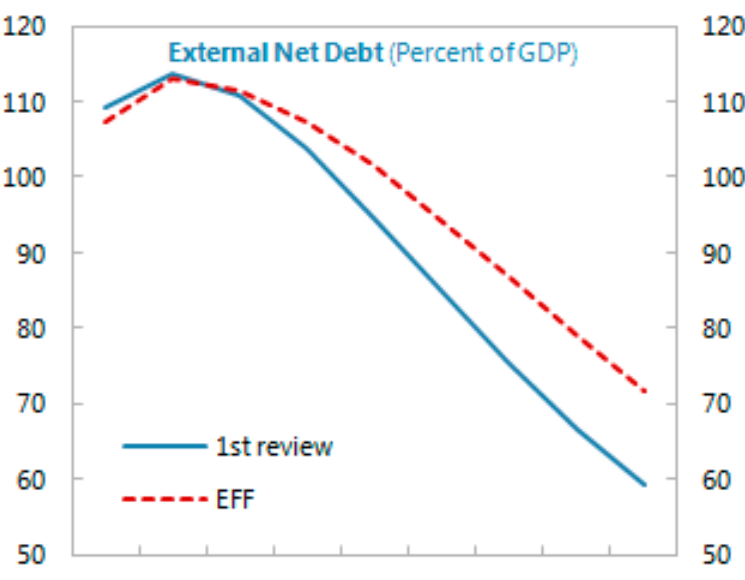

201220132014201520162017201820192020 Source: IMF staff projections. appears to be considerably more manageable. Compared to the Extended Arrangement, the debt service path is now significantly more benign. Interest payments in 2013-20 have been reduced by almost 3 percent of GDP per year in cash terms (falling below the euro area average), and principal repayments on debt falling due from 2020 to 2030 are reduced by an average of about 41/2 percent of GDP per year. The sharp decline in the debt service burden means that Greece's headline debtpeaking at about 180 percent of GDP in 2013-overstates the debt burden. To lend some perspective to this, the NPV of end-2012 debt evaluated at Greece's long-run projected nominal growth rate (around 4 percent) would be significantly lower, at 148 percent of GDP. This is also lower than the debt NPV at the time of EFF program approval (when it stood about 3 percentage 
points higher). Using a higher discount rate-in line with the range of borrowing rates within the euro area-would produce even lower debt NPVs.
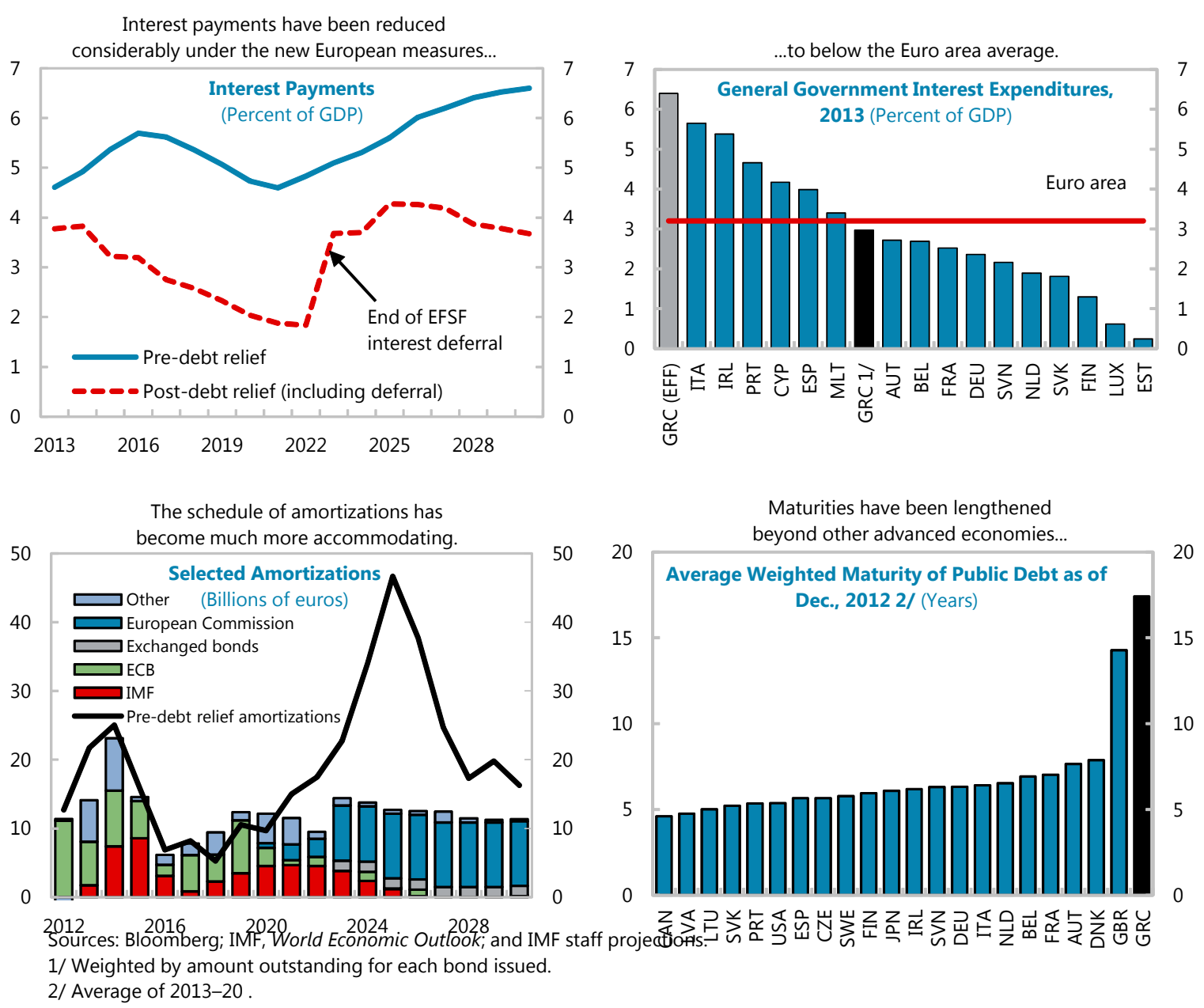

54. Greece also reached understandings with its European partners on additional financing for the program period (Tables 19 and 20). Greece's additional balance of payments financing needs over the period 2012-16 were estimated to be $€ 32$ billion. The debt buyback added about $€ 9.8$ billion to needs during $2012-16$ (with $€ 10.8$ billion upfront) while other debt-related measures (see $\llbracket 50$ ) reduced financing needs by about $€ 10$ billion over the same period. As outlined below, a total of roughly $€ 26$ billion in new financing was agreed, leaving a gap of €5.5-9.5 billion (depending on how transitory movements in deposits are accounted for), falling entirely in 2015-16:

- Greece's European partners agreed to a deferral and capitalization of EFSF interest payments falling due for one decade (providing an estimated $€ 11.5$ billion in financing through 2016).

- The ECB agreed, on a preliminary basis, to a rollover of maturing Greek bonds held by the national central banks (ANFA) (generating about $€ 5.6$ billion in further financing through 
2016). To the degree this rollover is not confirmed by March, the euro member states agreed to provide equivalent relief via other means.

At the request of its euro area partners, Greece agreed to delay the programmed $€ 9$ billion net redemption of Treasury bills now held by banks. With only a small share of the stock of Treasury bills expected to be eligible for use as collateral for BoG funding beyond Q1 2013, Greece's banks will need to make room for this through other adjustments in their balance sheets (likely involving higher ELA and higher access to ECB instruments which should be facilitated by the ECB's decision in mid-December 2012 to expand the list of eligible collateral).

55. Financing from Greece's European partners will be re-phased. Amounts have been brought forward to fill financing gaps in 2013. Disbursements will in general be tranched within quarters, linked to financing needs and the completion of objective milestones (defined in the program). Against this backdrop, following the $€ 34.3$ billion disbursed in December 2012, a further $€ 14.8$ billion will be disbursed in early January, early February, and early March 2013 (as Greece meets milestones related to, e.g., passage of the tax reform, and establishment of public sector staff reduction targets). The funds to be disbursed in the January-March period would include amounts for bank recap, which were delayed to provide resources to fund the debt buyback. It is important that these disbursements be frontloaded to January in a sufficient amount to minimize delays with bank restructuring and prevent tighter-thanprogrammed liquidity.

\begin{tabular}{lr}
\multicolumn{2}{c}{$\begin{array}{c}\text { E.U. Disbursements, 2012-14 } \\
\text { (Billions of euros) }\end{array}$} \\
\hline \multicolumn{2}{c}{ Amount } \\
\hline Disbursement & 49.1 \\
1st disbursement, 2012Q4 & 34.3 \\
Of which: & 14.8 \\
Dec., 2012 & 4.2 \\
Jan.-Mar., 2013 & 3.2 \\
2nd disbursement, 2013Q1 & 0.7 \\
3rd disbursement, 2013Q2 & 2.4 \\
4th disbursement, 2013Q3 & 6.3 \\
5th disbursement, 2013Q4 & 4.8 \\
6th disbursement, 2014Q1 & 0.0 \\
7th disbursement, 2014Q2 & 0.0 \\
8th disbursement, 2014Q3 & \\
9th disbursement, 2014Q4 &
\end{tabular}

Source: European Commission

56. IMF resources will also be re-phased (Table 21). The Greek authorities requested the purchases related to the first and second reviews, and a re-phasing of the undrawn third purchase, with the remaining outstanding access to be spread equally over all remaining reviews (on account of delays in program implementation). Each of the remaining purchases would equal SDR 1,506.7 million under the proposed re-phasing.

57. Financing assurances are in place for the review. The successful conclusion of the debt buyback, the various measures taken by Greece's European partners to reduce debt and provide financing, and the decisions taken by Europe to advance the EFSF disbursement schedule, ensure that the first year of the program remains fully financed and that there are firm commitments in place for financing for the second year. There also remain good prospects that financing will be available beyond the second program year, in particular to cover the $€ 91 / 2$ billion gap for 2015-16. Euro area members confirmed their previous commitment to provide adequate support to Greece during the period of the program and beyond, provided that Greece fully complies with the requirements and objectives of the program. 


\section{PROGRAM MODALITIES}

\section{Program Monitoring}

58. The authorities implemented ten prior actions to set the stage for Board consideration of the reviews (MEFP Table 3). These measures were geared to ensure that the program remained on track towards achieving its objectives:

- Recovering competitiveness and protecting real incomes. Prior actions in this area cover: (i) a package of liberalization measures in retail, fuel, transportation services, and regulated professions; (ii) a package of measures to reduce severance and other nonwage costs; and (iii) a package of reforms to eliminate legal obstacles to the privatization process. The authorities have developed many of the specific actions in cooperation with the European Commission staff. Staff views the collective package the authorities have assembled and passed in each area as macro critical (and the MEFP provides full information about the packages in Annexes I-III). Overall, these three actions will help recover competitiveness (by lowering input prices for businesses) and protect real incomes (by lowering final prices of consumer goods).

- $\quad$ Preserving financial stability. Prior actions cover: (i) steps to finalize the program for bank recapitalization; and (ii) measures to strengthen the governance of the financial system. These actions will help preserve the core of the financial sector in Greece, and ensure that proper checks and balances are in place to safeguard the significant resources provided under the program to recapitalize Greek banks.

- Restoring fiscal sustainability. Prior actions cover: (i) adoption of a package of revenue and expenditure measures to achieve the fiscal consolidation during 2012-14; (ii) steps to improve revenue administration; and (iii) improvement of commitment controls and arrears reporting. The authorities have developed many of the specific revenue and spending measures in cooperation with their technical assistance advisors and European Commission staff. Staff views the collective package the authorities have assembled and passed as macro critical (and the MEFP provides full information about the package in Annex V). The fiscal and fiscal institutional measures will indeed help ensure that medium-term fiscal targets are achieved, contributing to the restoration of fiscal sustainability.

59. Program quantitative targets are proposed for revision (MEFP Table 1b):

Fiscal quarterly performance criteria (QPCs). The floor on the modified general government primary cash balance, the ceiling on state budget primary spending, and the ceiling on the stock of domestic government arrears for end-December 2012 are all proposed to be adjusted to reflect understandings reached. These new targets are expected to be met, and the authorities are requesting waivers of applicability for the present reviews 
for these QPCs (data on these aggregates will not be available at the time of the Board meeting). The fiscal QPCs through end-June 2013 are also being established.

Indicative targets. The end-2012 indicative target on domestic government arrears is proposed to be adjusted to reflect the understandings reached with the authorities on arrears clearance. New indicative targets on domestic arrears and privatization proceeds are proposed for 2013, with one exception: for end-September 2013 the floor on privatization proceeds is proposed to be a QPC (to reflect the macro-criticality of progress in this area, and the need to allow some time for progress to be made).

Definitions. Three revisions are proposed: (i) to ensure a correct measurement of underlying fiscal progress, cash payments from loss-making banks will be excluded from central government primary revenue (TMU आ5); (ii) to facilitate a more comprehensive measure of arrears, verified tax refunds that have not been paid will be added to the program definition of domestic arrears (TMU I10); and (iii) to simplify the treatment of arrears, ceilings have been set on the stock, consistent with the programmed arrears clearance schedule, and arrears already subject to a QPC have been excluded from the primary balance QPC definition (TMU $\llbracket 6$ and $\llbracket 4$ ). Concerning arrears, while the change will help with monitoring, there will remain a risk of misreporting given weak Greek institutions.

60. The program incorporates a number of new structural benchmarks (MEFP Table 4):

To help restore competitiveness: (i) a strategy to reduce social security contribution rates will be adopted by end-November 2013 (reset benchmark from the Extended Arrangement); and (ii) the authorities will eliminate various small fees and taxes ("nuisance taxes") in the context of the 2014 budget (in September 2013). High and administratively burdensome taxation is a key impediment to competitiveness in Greece, motivating these benchmarks.

To preserve financial stability: (i) by end-January 2013, Hellenic Postbank will be resolved; (ii) by end-April 2013, all four core banks will be required to meet the capital adequacy requirements; (iii) by end-June 2013, all noncore banks that are undercapitalized or insolvent will be resolved; (iv) by end-July 2013, all banks should submit restructuring plans for validation to DG-Competition; and (v) by end-2013, the Bank of Greece will complete a follow-up stress test for all banks (to ensure that the recapitalization strategy has delivered the expected outcome). These benchmarks support the key forthcoming stages in the financial system restructuring process.

To secure fiscal sustainability: (i) by end-February 2013, a law to establish a semiautonomous tax agency will be adopted; (ii) by end-February 2013, the authorities will complete their staffing plans and specify targets for mandatory exits from the public sector; (iii) by end-June 2013, a new Tax Procedure Code will be adopted and the authorities will further simplify the text of the income tax law; and (iv) by end-2013, the Ministry of Finance will complete an audit of accounts payable to ensure compliance with arrears clearance 
targets and modalities. These benchmarks support structural changes necessary to secure the fiscal adjustment.

61. Program reviews will continue on a quarterly basis. The third and fourth reviews under the program are proposed to take place, respectively, on or after February 28 and May 31, 2013. The fifth review, in addition to the various applicable program conditions, will have a focus on overall progress with tax administration reforms, while the sixth review will include a focus on overall progress with privatization (again in addition to various other applicable program conditions).

\section{Exceptional access criteria}

\section{The program continues to satisfy the substantive criteria for exceptional access but} with little to no margin. Developments in the last six months raised issues about debt sustainability and the capacity of the authorities to implement the program in a difficult political environment. Nevertheless, the additional efforts undertaken by the authorities and their European partners in the form of strong prior actions, modified program commitments, and the debt relief allow the program to satisfy the criteria for exceptional access, although still with little to no margin. The continued commitment of euro area member states to support Greece and to undertake further debt relief as necessary is an essential part of the argument in favor of meeting the criteria:

Criterion 1 The member is experiencing or has the potential to experience exceptional balance of payments pressures on the current or capital account, resulting in a need for Fund financing that cannot be met within the normal limits. Financial conditions are projected to remain unfavorable in the foreseeable future as deleveraging proceeds; and sovereign spreads are expected to remain elevated for a long period until Greece establishes a track record and decisively reduces debt. Against this background, balance of payments support beyond normal access limits continues to be required to meet Greece's large external financing needs.

Criterion 2 A rigorous and systematic analysis indicates that there is a high probability that the member's public debt is sustainable in the medium term. However, in instances where there are significant uncertainties that make it difficult to state categorically that there is a high probability that the debt is sustainable over this period, exceptional access would be justified if there is a high risk of international systemic spillover. The baseline debt trajectory built into the program is sustainable in the medium term but still subject to significant downside risks. Strict program implementation and, crucially, additional official debt relief will eventually deliver significant debt reduction and a significant reduction in debt service costs placing debt on a firmly declining trajectory. However, near term uncertaintiescaptured in a debt peak of near 180 percent of GDP_remain high enough to make it difficult to categorically affirm that debt is sustainable with high probability. At the current juncture, however, it is not possible to establish that contagion risk from Greece would be limited and manageable in the event the program review with Greece did not proceed (likely precipitating a euro exit). Notwithstanding falling exposure of the private sector to Greece 
and the stronger European firewall, models suggest that Greek exit could result in large and persistent output losses in Europe (Box 2). The rally in European peripheral markets after the agreement on Greece was reached and the debt buyback was concluded does indeed suggest that markets see a strong link to Greece. Exceptional access is thus justified given the still-high risk of international systemic spillovers.

Criterion 3 The member has prospects for gaining or regaining access to private capital markets within the timeframe when Fund resources are outstanding. Greece does not have market access, but conditional on successful program implementation and adequate support from its European partners, there are adequate prospects for regaining market access. Projecting the scale and timing of Greece's return to private capital markets is difficult given the unprecedented levels of official debt compared to the standards of market access countries and the inherent complications of estimating the economy's response to reforms. Consequently, market access assumptions in the post-program period are conservative and based on initially small issuances at short maturities and high spreads. This approach to restoring market access is expected to require protracted and significant official support to avoid the adverse debt dynamics and risk of market access loss that would result from exclusive reliance on market financing. Euro area member states have in fact agreed to extend the maturities of their GLF and EFSF loans, which vastly reduces Greece's debt service costs and rollover requirements, considerably enhancing the prospect for Greece to gradually return to markets. They have also reiterated their commitment to provide financing during the program period and beyond until Greece restores market access, provided that Greece implements the program in full. Thus, this financing strategy ensures that Greece will have financing in a scale and timing adequate to secure repayment of Fund resources.

Criterion 4 The policy program of the member provides reasonably strong prospect of success, including not only the member's adjustment plans but also its institutional and political capacity to deliver that adjustment. Political instability, persistent social pressures, and administrative capacity constraints tested the authorities at the outset of the Extended Arrangement, and implementation of the authorities' extremely challenging policy program fell well behind schedule. However, the authorities have since demonstrated program ownership and policy resolve through the implementation of a set of prior actions that delivered strong results in key areas. The program is also being substantially revised in the present reviews to reduce administrative capacity challenges-for instance by extending timelines for implementation-and continued technical assistance from the EC and IMF will also support the authorities' efforts to improve their institutional and technical capacity. On this basis, and although implementation risks remain, staff assesses there to be a reasonably strong prospect of success of the program. 


\section{Risks and mitigation}

63. Risks to the program are still on the downside. Greece is attempting to achieve an unprecedented amount of fiscal and current account adjustment under a fixed exchange rate, with a massive debt overhang, and weak confidence. Key risks include:

- $\quad$ Political failure. A key risk for the program is diminishing support for reforms, particularly as Greece endures another year of deep recession. The latest opinion polls show dwindling support for the coalition parties and growing support for Syriza and other anti-program parties. This could go beyond implementation delays, and lead to a political crisis, triggering debt default and/or euro exit.

- Delayed recovery of confidence. Even without a complete political failure, political instability and the risk of disorderly default/euro exit may have a stronger effect on confidence and investment than now projected. In this instance, growth would undershoot projections, and fiscal adjustment would struggle against these headwinds. These impacts could make the process self-reinforcing, leaving Greece in a high-debt low-growth trap.

- Transmission mechanism. The program now takes a more conservative view on fiscal multipliers, but they could yet be higher than currently projected, especially if the external environment is weaker, or if bank deleveraging proceeds at a more rapid than expected pace (e.g., due to crowding out, as treasury bills are retained on banks' balance sheets). Separately, gains from structural reforms remain uncertain, as it could take longer for wage adjustment to translate into price adjustment, given still strong vested interests, and it could take longer for stronger competitiveness to translate into higher net exports.

\section{While the program aims to mitigate these risks, it is clear that if they do play out,} additional debt relief and financing would be needed from Greece's European partners. The program aims to limits risks via upfront policy implementation (to help put many difficult actions behind the government), tighter fiscal rules (to help prevent future deviations), protection for the most vulnerable (to help contain political stresses), and reinforcement of structural reform efforts (to lay a basis for stronger growth). If macro risks play out-particularly the confidence and transmission mechanism concerns detailed above-then Greece would need more time to adjust, supported by additional debt relief and financing from Greece's European partners. The alternative scenario considered in the DSA gives some indication of the potential needs. In this scenario, to return Greece to the program debt trajectory would require about 3 percent of Greek GDP per year (€6 billion) in fiscal transfers between 2013 and 2020 (which could be delivered through a variety of channels, including lower interest rates on GLF and EFSF loans). Alternatively, it would require an upfront haircut of about 25 percent on EFSF loans, GLF loans, and ECB SMP bond holdings. 


\section{Capacity to repay and safeguards}

\section{Under the baseline, the authorities have the capacity to repay the Fund, but this would not be the case if the program went irretrievably off-track (Table 22):}

- Under the revised program baseline, the macroeconomic outlook is projected to be weaker, but an official support package from euro area member states that provides debt relief and support to restore market access will help to keep debt on a sustained downward path. With lower GDP, total debt service to the Fund will peak at about 5 percent of GDP in 2015, compared to $4 \frac{1}{2}$ percent under the EFF-supported program. Peak access projections remain broadly unchanged from the program request (2,501 percent of quota). Still, capacity to repay the Fund critically depends on euro area member states commitment to provide support to Greece during the program and beyond. And since this is conditional on Greece's adherence to all program policies, capacity to repay the Fund will also depend on the authorities' ability to implement the ambitious program in full.

- If the program were to go irretrievably off-track, and the euro area member states voided their commitment to continue to support Greece, the country would likely not have the capacity to repay the Fund. As noted, long-term European support is critical to help Greece restore market access. The Fund's longstanding de facto preferred creditor status would continue to provide some protection against financial risks. Still, the Fund's capacity to absorb any resulting losses would be limited since credit outstanding to Greece would surpass 200 percent of projected Fund precautionary balances in 2013, and since low SDR interest rates exacerbate the limitations of burden sharing capacity.

\section{An updated safeguards assessment of the Bank of Greece shows that some key} safeguards functions are still under development. Emergency lending operations are subject to adequate regulatory and control frameworks but an internal compliance review by the BoG is warranted. In light of significant ELA exposure, the assessment recommended that the BoG conduct an analysis of the legal aspects that would arise in cases of a need for recapitalization or indemnification of losses by the State. Greece has also, since the time of the safeguards assessment, put into place legal arrangements to channel targeted primary surpluses (plus 30 percent of primary surpluses in excess of targets) and privatization revenues into the segregated account, which may be used only for payment of debt service (to all creditors). This action, which is intended to help ensure timely debt service, does not pose any safeguards issues for the Fund. 


\section{STAFF APPRAISAL}

67. The Greek program continues to present a mixed picture with stark contrasts. The fiscal adjustment has been extraordinary by any measure and labor market reforms have produced significant wage adjustment. There should be no doubt on part of Greece's European partners that it is enduring exceptionally painful adjustment in order to stay within the euro area. However, the manner of the adjustment leaves much to be desired. The fiscal adjustment has relied far too much on cuts in discretionary spending and increased taxation of wage earners, while the rich and selfemployed have continued to evade taxes on an astonishing scale and bloated and unproductive state sectors have seen only limited cuts. Moreover, labor has shouldered too much of the burden as lower wages have not resulted in lower prices, because of failure to liberalize closed professions and dismantle barriers to competition. While the economy is now re-balancing apace, this is happening mainly through recessionary channels, rather than through productivity boosting reforms. Meanwhile, the mounting sense of social unfairness is undermining support for the program.

\section{The new government acknowledges that the program will fail unless it overcomes} these entrenched vested interests. In all key policy areas, its main objectives and plans are generally aligned with those advocated by staff. But while it has already delivered important upfront changes in some areas, it still needs to demonstrate political resolve in some of the most difficult ones.

69. Fiscal efforts remain very impressive. The scale of the frontloaded adjustment is evidence of the government's strong determination to bring the program back on track. The refocusing on cuts in wages and social transfers has no doubt been socially and politically painful, but this was overdue as the surge in wages and, in particular, pensions was the main reason for the rise in the fiscal deficit following euro adoption. In order for the government to deliver on its promise to avoid further wage and pension cuts, it is critical that it soon achieve efficiency savings via significant mandatory redundancies, and that it move to radically overhaul the corrupt and ineffective tax administration. The government's achievements in these two areas have so far not been commensurate with its ambitious goals. Going forward, tackling tax evasion will be a litmus test for the support of the program, both within Greece and among Greece's European partners.

70. Progress is being made in reforming labor and product markets. As the impact of labor market reforms to date has been considerable, staff accepts delaying agreed reforms of the minimum wage regime, although it continues to consider such reforms to be essential. Meanwhile, the cut in severance pay is an important step towards reducing nonwage costs. Important upfront actions have also been taken to open up closed professions and promote product market reforms, but Greece is still well away from the critical mass of reforms needed to unlock sustained productivity gains, lower prices and protect real incomes. This will be another key area where the government's resolve will be tested as most of the difficult reforms that still lie ahead will challenge constituencies traditionally aligned with the main governing party. 
71. Privatization rhetoric needs to be matched with results. Greece needs to move decisively away from its previous state-led growth model, and privatization is key to this. Results to date have been extremely disappointing. Staff welcomes the government's stated intention to accelerate privatization, and while there is no significant evidence of this so far it accepts that it will take time to restart the stalled process and that market conditions are unfavorable. Still, staff remains concerned about the robustness of the privatization process to political interference-the agreement to require the privatization agency to consult with parliament have heightened such concerns-and about its ability to resist giving preference to insiders. Should the planned mid-year review of the privatization process show that the problems persist, the government should consider radical governance changes, including the replacement of current managers by foreign experts.

72. Good progress is being made in stabilizing the financial sector. Staff welcomes the finalization of the recapitalization framework. Looking ahead, the almost complete removal of direct sovereign exposure from banks' balance sheets has significantly reduced risks. However, with the state set to take a major stake in all key banks, it is critical that the Government refrains from interfering in day-to-day management, while the HFSF takes steps to protect public interests. In this regard, staff welcomes the establishment of an enhanced monitoring and supervisory framework. With so much in public resources on the line, it will be important for the EC and ECB to use the powers made available to them to exert effective oversight, alongside Greek supervision agencies.

\section{Greece's European partners are taking politically difficult steps to ease its debt burden.}

The agreed upfront measures are an important first step, but staff projections suggest that they will be insufficient to keep debt close to the targeted trajectory. Staff, therefore, welcomes the assurances to take additional measures in 2014 and 2015-provided that Greece adheres to its primary deficit targets-to reduce debt to 124 percent of GDP by 2020 and substantially below 110 percent by 2022. Upfront measures to achieve a reduction to the original target of 120 percent by 2020 would have been preferable, as would a specification of the exact modalities for future relief. Still, staff recognizes that the assurances concerning future conditional relief given in the context of the present review reflects an explicit acknowledgement, for the first time since the onset of the crisis, that Greece's debt burden is unsustainable without long-term transfers. From this perspective, Greece and its European partners have turned an important corner.

\section{The extension of repayment periods on GLF and EFSF loans has significant long-term} benefits. In combination with the buyback of much of the privately held bonds, the extension has sharply reduced what would otherwise have been a surge in refinancing needs starting in 2022 . This has an important impact on debt-to-GDP over the very long run, as Greece will carry low-interestrate debt to its European partners for much longer than previously assumed. This positive long-term impact on refinancing needs and debt, which should facilitate Greece's return to markets, is further evidence of European partners putting in place a credible framework for a lasting solution to Greece's debt problem.

75. Financing constraints remain a concern. The program is fully financed on a 12-month forward looking basis, and the proposal to roll-over maturing bonds held by Eurosystem Central 
Banks could provide sufficient financing assurances through end-2013. Greece's European partners would have to commit to new funding at this point by the latest. On a related matter, financing constraints mean that the government is no longer planning to reduce its T-bill exposure by $€ 9$ billion in 2013, contrary to what was assumed in the program. With no accommodation by the $E C B$, this suggests that liquidity conditions will be notably tighter. If this is judged to pose a significant risk to the macro framework, staff would expect euro area member states to provide additional financing in 2013 to allow Greece to reduce its T-bills exposures as originally programmed, or for the ECB to raise its limit on repo-eligible T-bills.

76. The gradual and contingent approach to debt relief will need to be revisited if the high debt burden continues to weigh on investments. The assumption that GDP will start to recover in 2014 — when fiscal consolidation is still exerting a notable drag —is based on the expectation that a gradual recovery in confidence and in investments will begin to take hold by then. Important in this regard, Staff believes that recent evidence that the authorities are determined to persevere with the program, despite a difficult political situation, and that European partners are continuing to provide unprecedented support, will gradually convince investors that the risk of euro exit is dissipating, despite the high debt levels entailed by the gradual approach to debt relief. Should it become evident, however, that investors' confidence remains subdued because of concerns about the debt burden-despite strong program implementation by the authorities-a more frontloaded approach to debt relief would need to be considered. The macro and debt outlook will therefore need to be assessed carefully at each future review.

\section{Overall, the program is moving in the right direction, but the challenges ahead remain} enormous. The program continues to be the best opportunity for Greece to successfully complete its adjustment within the euro area. In this regard, even with generous debt relief, Greece will not successfully restore robust growth while remaining in the euro area without deep structural reforms. On the basis of the government's policy commitments, and the commitment of Greece's European partners to support Greece during the program and beyond, including with deep relief to achieve debt sustainability, staff can support the completion of the first and second reviews and the authorities' request for modification and waivers of applicability of the end-December 2012 PCs, modification of the PCs and re-phasing of purchases under the arrangement. 


\begin{tabular}{|c|c|c|c|c|c|c|c|c|}
\hline \multicolumn{9}{|c|}{ Table 1. Greece: Selected Economic Indicators, 2007-13 } \\
\hline & \multirow[t]{2}{*}{2007} & \multirow[t]{2}{*}{2008} & \multirow[t]{2}{*}{2009} & \multirow[t]{2}{*}{2010} & \multirow[t]{2}{*}{2011} & \multicolumn{2}{|c|}{2012} & \multirow{2}{*}{$\begin{array}{l}2013 \\
\text { Proj. }\end{array}$} \\
\hline & & & & & & Prog. & Proj. & \\
\hline & \multicolumn{8}{|c|}{ (Percentage change, unless otherwise indicated) } \\
\hline \multicolumn{9}{|l|}{ Domestic economy } \\
\hline Real GDP & 3.5 & -0.2 & -3.1 & -4.9 & -7.1 & -4.8 & -6.0 & -4.2 \\
\hline Output gap (percent of pot. output) & 10.0 & 9.9 & 7.3 & 3.3 & -2.6 & -6.7 & -7.3 & -10.2 \\
\hline Total domestic demand & 6.2 & -0.2 & -5.5 & -7.0 & -8.7 & -6.7 & -8.7 & -6.5 \\
\hline Private consumption & 3.6 & 4.3 & -1.6 & -6.2 & -7.7 & -5.7 & -7.7 & -6.9 \\
\hline Public consumption & 7.1 & -2.6 & 4.9 & -8.7 & -5.2 & -11.0 & -6.2 & -7.2 \\
\hline Gross fixed capital formation & 22.8 & -14.3 & -13.7 & -15.0 & -19.6 & -6.6 & -14.4 & -3.3 \\
\hline Change in stocks (contribution) & -1.9 & 0.6 & -1.2 & 0.7 & -0.4 & 0.0 & 0.4 & 0.0 \\
\hline Foreign balance (contribution) & -3.3 & 0.1 & 3.0 & 3.0 & 2.4 & 2.5 & 3.3 & 2.5 \\
\hline Exports of goods and services & 7.1 & 1.7 & -19.4 & 5.2 & 0.3 & 3.2 & -1.2 & 2.7 \\
\hline Imports of goods and services & 14.5 & 0.9 & -20.2 & -6.2 & -7.3 & -5.1 & -11.5 & -6.2 \\
\hline Unemployment rate (percent) $1 /$ & 8.3 & 7.7 & 9.4 & 12.5 & 17.5 & 19.1 & 24.4 & 26.6 \\
\hline Employment 1/ & 1.2 & 1.1 & -1.0 & -2.7 & -6.6 & -4.8 & -9.2 & -3.9 \\
\hline Unit labor costs & 1.3 & 0.4 & 4.2 & -2.6 & -3.4 & -8.6 & -8.1 & -6.6 \\
\hline Consumer prices (HICP), end of period & 4.0 & 2.0 & 2.6 & 5.2 & 2.4 & 0.8 & 1.3 & -0.6 \\
\hline Consumer prices (HICP), period average & 2.9 & 4.2 & 1.2 & 4.7 & 3.3 & -0.5 & 1.2 & -0.8 \\
\hline Core prices, period average $2 /$ & 3.1 & 3.1 & 2.3 & 2.6 & 1.1 & $\ldots$ & $\ldots$ & $\ldots$ \\
\hline \multirow[t]{2}{*}{ GDP deflator } & 3.3 & 4.7 & 2.3 & 1.1 & 1.0 & -0.7 & -0.5 & -1.2 \\
\hline & \multicolumn{8}{|c|}{ (Percent of GDP, unless otherwise indicated) } \\
\hline \multicolumn{9}{|l|}{ Balance of payments } \\
\hline Current account & -14.6 & -14.9 & -11.2 & -10.1 & -9.9 & -7.5 & -4.2 & -1.2 \\
\hline Trade balance & -11.2 & -11.5 & -7.8 & -6.8 & -6.0 & -4.2 & -2.5 & -0.3 \\
\hline Export of goods and services & 21.9 & 23.1 & 18.3 & 20.5 & 23.4 & 24.8 & 25.3 & 27.5 \\
\hline Imports of goods and services & -33.0 & -34.6 & -26.2 & -27.3 & -29.5 & -29.1 & -27.8 & -27.7 \\
\hline Total transfers & 0.7 & 1.2 & 0.6 & 0.1 & 0.3 & 0.3 & 0.3 & 1.8 \\
\hline Net income receipts & -4.2 & -4.6 & -3.9 & -3.5 & -4.1 & -3.6 & -2.0 & -2.7 \\
\hline Net international investment position & -96.1 & -76.8 & -89.6 & -98.4 & -86.1 & -125.0 & -94.2 & -97.6 \\
\hline \multicolumn{9}{|l|}{ Public finances (general government) } \\
\hline Total revenues & 40.7 & 40.6 & 38.3 & 40.6 & 42.2 & 42.2 & 43.6 & 42.9 \\
\hline Total expenditures & 47.5 & 50.5 & 53.9 & 51.4 & 51.7 & 49.5 & 50.3 & 47.4 \\
\hline Primary expenditures & 42.7 & 45.4 & 48.8 & 45.4 & 44.5 & 43.2 & 45.1 & 42.9 \\
\hline Overall balance & -6.8 & -9.9 & -15.6 & -10.7 & -9.4 & -7.3 & -6.7 & -4.5 \\
\hline Primary balance & -2.0 & -4.8 & -10.4 & -4.8 & -2.2 & -1.0 & -1.5 & 0.0 \\
\hline Gross debt & 107 & 112 & 129 & 148 & 171 & 163 & 158 & 178 \\
\hline \multicolumn{9}{|l|}{ Interest rates and credit } \\
\hline Lending interest rate (percent) 3/ & 6.7 & 6.7 & 5.1 & 6.1 & 6.8 & $\ldots$ & 5.8 & $\ldots$ \\
\hline Private credit growth (percent change) 4 / & 21.5 & 15.9 & 4.2 & 0.0 & -3.1 & -4.0 & -7.2 & -3.1 \\
\hline \multicolumn{9}{|l|}{ Exchange rates (percent change) } \\
\hline Nominal effective exchange rate $3 /$ & 1.9 & 2.1 & 0.4 & -3.7 & 0.0 & $\ldots$ & -2.1 & $\ldots$ \\
\hline Real effective exchange rate (CPI-based) $3 /$ & 2.1 & 2.2 & 1.7 & -1.2 & -0.6 & $\ldots$ & -3.0 & $\ldots$ \\
\hline Real effective exchange rate (man. ULC-based) & 2.5 & -1.5 & 0.2 & -1.1 & -0.3 & $\ldots$ & $\ldots$ & $\ldots$ \\
\hline \multicolumn{9}{|l|}{ Memorandum items: } \\
\hline Nominal GDP (billions of euros) & 223 & 233 & 231 & 222 & 209 & 204 & 195 & 185 \\
\hline Nominal GDP (percent change) & 7.0 & 4.5 & -0.9 & -3.9 & -6.1 & -5.4 & -6.5 & -5.4 \\
\hline \multicolumn{9}{|c|}{ Sources: National Statistical Service; Ministry of Economy and Finance; Bank of Greece; and IMF staff projections. } \\
\hline 1/ Based on Labor Force Survey. & & & & & & & & \\
\hline 2/ Core prices exclude energy, food, alcohol, an & & & & & & & & \\
\hline 3/ Data for 2012 as of October. & & & & & & & & \\
\hline $\begin{array}{l}\text { 4/ Includes securitized or otherwise transferred } \\
\text { reclassifications. Data for } 2012 \text { as of March. }\end{array}$ & 2010 & ard. Pro & ons de & ake & our & -offs, v & tion ch & $s$, or \\
\hline
\end{tabular}


Table 2. Greece: General Government: Statement of Operations

(GFSM 2001, flows), 2008-11

(Millions of euros)

\begin{tabular}{|c|c|c|c|c|}
\hline & 2008 & 2009 & 2010 & 2011 \\
\hline Revenue & 94,848 & 88,602 & 90,232 & 88,188 \\
\hline Taxes & 48,297 & 45,823 & 45,109 & 44,916 \\
\hline Social contributions & 30,749 & 29,457 & 29,764 & 27,492 \\
\hline Grants & 939 & 659 & 614 & 493 \\
\hline Other revenue & 14,863 & 12,663 & 14,745 & 15,287 \\
\hline Expenditure & 117991 & 124669 & 114302 & 108003 \\
\hline Expense & 113,648 & 122,070 & 114,485 & 111,186 \\
\hline Compensation of employees & 28,000 & 31,010 & 27,773 & 25,938 \\
\hline Use of goods and services & 15,046 & 17,111 & 13,679 & 9,881 \\
\hline Consumption of fixed capital & 4,613 & 5,017 & 5,386 & 5,782 \\
\hline Interest & 11,937 & 11,917 & 13,193 & 15,024 \\
\hline Subsidies & 70 & 123 & 129 & 119 \\
\hline Grants & 2,209 & 2,155 & 2,020 & 1,866 \\
\hline Social benefits & 45,758 & 48,972 & 47,220 & 47,210 \\
\hline Other expense & 6,015 & 5,765 & 5,085 & 5,366 \\
\hline Net acquisition of nonfinancial assets & 4,343 & 2,599 & -183 & $-3,183$ \\
\hline Acquisitions of nonfinancial assets & $\ldots$ & $\ldots$ & $\ldots$ & $\ldots$ \\
\hline Disposals of nonfinancial assets & $\ldots$ & $\ldots$ & $\ldots$ & $\ldots$ \\
\hline Consumption of fixed capital & 4,613 & 5,017 & 5,386 & 5,782 \\
\hline Gross operating balance $\mathbf{1 /}$ & $-14,187$ & $-28,451$ & $-18,867$ & $-17,216$ \\
\hline Net operating balance 2/ & $-18,800$ & $-33,468$ & $-24,253$ & $-22,998$ \\
\hline Net lending (+)/borrowing (-) 3/ & $-23,143$ & $-36,067$ & $-24,070$ & $-19,815$ \\
\hline Net acquisition of financial assets & 8,687 & 2,280 & 4,864 & 330 \\
\hline Monetary gold and SDRs & 0 & 0 & 0 & 0 \\
\hline Currency and deposits & 4,093 & $-1,384$ & 5,101 & $-2,010$ \\
\hline Debt securities & 4,406 & 40 & 0 & -48 \\
\hline Loans & -361 & 3,670 & 146 & 827 \\
\hline Equity and investment fund shares & -278 & -120 & 362 & 646 \\
\hline Insurance, pensions, and standardized guarantee schemes & -1 & 3 & 3 & 2 \\
\hline Financial derivatives and employee stock options & 0 & 0 & 0 & 0 \\
\hline Other accounts receivable & 828 & 71 & -748 & 913 \\
\hline Net incurrence of liabilities & 31,334 & 38,595 & 28,735 & 20,389 \\
\hline SDRs & 0 & 0 & 0 & 0 \\
\hline Currency and deposits & 38 & 722 & -503 & -185 \\
\hline Debt securities & 25,015 & 37,920 & -310 & $-11,192$ \\
\hline Loans & 1,099 & $-1,108$ & 30,222 & 35,593 \\
\hline Equity and investment fund shares & 0 & 0 & 0 & 0 \\
\hline Insurance, pensions, and standardized guarantee schemes & 0 & 0 & 0 & 0 \\
\hline Financial derivatives and employee stock options & 207 & -522 & 951 & 166 \\
\hline Other accounts payable & 4,975 & 1,583 & $-1,625$ & $-3,993$ \\
\hline
\end{tabular}

Source: IMF, Government Finance Statistics.

1 / Revenue minus expense (excluding consumption of fixed capital).

2/ Revenue minus expense (including consumption of fixed capital).

3/ Revenue minus expenditure. 
Table 3. Greece: Financial Balance Sheet (GFSM 2001, stocks), 2008-11

(Millions of euros)

\begin{tabular}{|c|c|c|c|c|}
\hline & 2008 & 2009 & 2010 & 2011 \\
\hline \multicolumn{5}{|l|}{ Stock positions } \\
\hline Net worth & $\ldots$ & $\ldots$ & $\ldots$ & $\ldots$ \\
\hline Nonfinancial assets & $\ldots$ & $\ldots$ & $\ldots$ & $\ldots$ \\
\hline Net financial worth & $-221,098$ & $-242,315$ & $-211,031$ & $-149,654$ \\
\hline Financial assets & 64,470 & 77,248 & 79,571 & 75,838 \\
\hline Monetary gold and SDRs & 0 & 0 & 0 & 0 \\
\hline Currency and deposits & 13,271 & 11,762 & 16,905 & 14,741 \\
\hline Debt securities & 702 & 741 & 741 & 692 \\
\hline Loans & 1,591 & 5,261 & 5,408 & 6,234 \\
\hline Equity and investment fund shares & 29,256 & 39,757 & 37,533 & 34,270 \\
\hline Insurance, pensions, and standardized guarantee schemes & 38 & 42 & 47 & 48 \\
\hline Financial derivatives and employee stock options & 0 & 0 & 0 & 0 \\
\hline Other accounts receivable & 19,612 & 19,685 & 18,937 & 19,853 \\
\hline Liabilities & 285,568 & 319,563 & 290,602 & 225,492 \\
\hline Monetary gold and SDRs & 0 & 0 & 0 & 0 \\
\hline Currency and deposits & 786 & 1,508 & 1,005 & 820 \\
\hline Debt securities & 213,616 & 248,183 & 190,633 & 97,285 \\
\hline Loans & 45,912 & 44,791 & 75,177 & 106,770 \\
\hline Equity and investment fund shares & 0 & 0 & 0 & 0 \\
\hline Insurance, pensions, and standardized guarantee schemes & 0 & 0 & 0 & 0 \\
\hline Financial derivatives and employee stock options & 2,736 & 980 & 1,311 & 2,134 \\
\hline Other accounts payable & 22,518 & 24,101 & 22,476 & 18,483 \\
\hline \multicolumn{5}{|l|}{ Memorandum items: } \\
\hline Publicly guaranteed debt & $\ldots$ & $\ldots$ & $\ldots$ & $\ldots$ \\
\hline Debt (at market value) & 282,832 & 318,583 & 289,291 & 223,358 \\
\hline Debt at face value & 277,209 & 314,674 & 342,908 & 365,186 \\
\hline Maastricht debt & 263,284 & 299,682 & 329,513 & 355,658 \\
\hline Debt (at nominal value) & $\ldots$ & $\ldots$ & $\ldots$ & $\ldots$ \\
\hline \multicolumn{5}{|l|}{ Other economic flows } \\
\hline Change in net worth from other economic flows & $\ldots$ & $\ldots$ & $\ldots$ & $\ldots$ \\
\hline Nonfinancial assets & $\ldots$ & $\ldots$ & $\ldots$ & $\ldots$ \\
\hline Change in net financial worth from other economic flows & $-5,401$ & 15,098 & 55,156 & 81,437 \\
\hline Financial assets & $-17,440$ & 10,498 & $-2,540$ & $-3,562$ \\
\hline Monetary gold and SDRs & 0 & 0 & 0 & 0 \\
\hline Currency and deposits & 49 & -125 & 42 & -153 \\
\hline Debt securities & $-4,026$ & -1 & 0 & 499 \\
\hline Loans & 0 & 0 & 1 & 0 \\
\hline Equity and investment fund shares & $-13,462$ & 10,621 & $-2,586$ & $-3,910$ \\
\hline Insurance, pensions, and standardized guarantee schemes & 0 & 1 & 1 & -1 \\
\hline Financial derivatives and employee stock options & 0 & 0 & 0 & 0 \\
\hline Other accounts receivable & -1 & 2 & 2 & 3 \\
\hline Liabilities & $-12,039$ & $-4,600$ & $-57,696$ & $-84,999$ \\
\hline Monetary gold and SDRs & 0 & 0 & 0 & 0 \\
\hline Currency and deposits & 0 & 0 & 0 & 0 \\
\hline Debt securities & $-11,095$ & $-3,353$ & $-57,240$ & $-81,656$ \\
\hline Loans & 6 & -13 & 164 & $-4,000$ \\
\hline Equity and investment fund shares & 0 & 0 & 0 & 0 \\
\hline Insurance, pensions, and standardized guarantee schemes & 0 & 0 & 0 & 0 \\
\hline Financial derivatives and employee stock options & -950 & $-1,234$ & -620 & 657 \\
\hline Other accounts payable & 0 & 0 & 0 & 0 \\
\hline
\end{tabular}

Source: IMF, Government Finance Statistics. 
Table 4. Greece: Modified General Government Cash Balance, 2012-16

\begin{tabular}{|c|c|c|c|c|c|c|c|c|}
\hline & \multirow[t]{3}{*}{2012} & \multicolumn{4}{|c|}{2013 (cumulative) } & \multirow[t]{3}{*}{2014} & \multirow[t]{3}{*}{2015} & \multirow{3}{*}{$\begin{array}{l}2016 \\
\text { Proj. }\end{array}$} \\
\hline & & \multirow[t]{2}{*}{ Mar-13 } & \multirow[t]{2}{*}{ Jun-13 } & \multirow{2}{*}{\multicolumn{2}{|c|}{$\begin{array}{l}\text { Sep-13 Dec-13 } \\
\text { Prog. }\end{array}$}} & & & \\
\hline & & & & & & & & \\
\hline \multicolumn{9}{|l|}{ I. State budget } \\
\hline Revenue & 52.3 & 11.4 & 22.2 & 35.5 & 51.3 & 50.4 & 50.6 & 51.9 \\
\hline Ordinary budget $(A+B+C-D)$ & 47.6 & 10.4 & 20.8 & 32.6 & 46.3 & 43.9 & 44.6 & 46.3 \\
\hline A. Recurrent revenue & 49.6 & 10.8 & 21.6 & 33.8 & 47.8 & 45.7 & 46.6 & 48.4 \\
\hline 1. Direct taxes & 20.8 & 4.1 & 8.6 & 14.3 & 20.6 & 18.7 & 18.8 & 19.3 \\
\hline Income taxes & 13.0 & 2.2 & 4.8 & 8.4 & 11.7 & 11.9 & 12.3 & 13.3 \\
\hline PIT & 9.8 & 1.9 & 3.7 & 6.2 & 8.7 & 8.8 & 9.0 & 9.6 \\
\hline CIT & 1.6 & 0.0 & 0.5 & 1.1 & 1.6 & 1.7 & 1.8 & 2.2 \\
\hline Other & 1.5 & 0.3 & 0.6 & 1.1 & 1.4 & 1.4 & 1.5 & 1.5 \\
\hline Property taxes & 2.8 & 0.2 & 0.9 & 1.9 & 3.2 & 2.9 & 2.9 & 2.9 \\
\hline Tax arrears collection & 2.1 & 1.0 & 1.7 & 2.2 & 3.2 & 1.5 & 1.5 & 1.7 \\
\hline Other direct taxes & 2.9 & 0.7 & 1.1 & 1.8 & 2.5 & 2.5 & 2.1 & 1.4 \\
\hline 2. Indirect taxes & 26.2 & 6.3 & 11.9 & 17.9 & 25.0 & 24.6 & 25.2 & 26.4 \\
\hline Transaction taxes & 15.7 & 3.8 & 7.3 & 10.9 & 14.6 & 14.5 & 14.8 & 15.6 \\
\hline VAT & 15.1 & 3.7 & 7.0 & 10.5 & 14.0 & 13.8 & 14.1 & 14.9 \\
\hline Other & 0.7 & 0.1 & 0.3 & 0.4 & 0.6 & 0.7 & 0.7 & 0.7 \\
\hline Consumption taxes & 9.7 & 2.4 & 4.3 & 6.5 & 9.6 & 9.4 & 9.6 & 10.0 \\
\hline Tax arrears collections & 0.5 & 0.1 & 0.2 & 0.3 & 0.4 & 0.4 & 0.4 & 0.5 \\
\hline Other indirect taxes & 0.3 & 0.1 & 0.1 & 0.2 & 0.3 & 0.3 & 0.3 & 0.3 \\
\hline 3. Transfers EU & 0.2 & 0.0 & 0.1 & 0.1 & 0.2 & 0.3 & 0.3 & 0.3 \\
\hline 4. Nontax revenue & 2.4 & 0.4 & 1.0 & 1.5 & 2.0 & 2.2 & 2.4 & 2.5 \\
\hline B. One-off revenue & 1.7 & 0.2 & 0.5 & 0.8 & 1.1 & 0.9 & 0.9 & 1.0 \\
\hline C. Revenue from concession and rights & 0.0 & 0.0 & 0.0 & 0.0 & 0.1 & 0.2 & 0.0 & 0.0 \\
\hline D. Tax refunds & 3.7 & 0.6 & 1.4 & 2.0 & 2.7 & 2.9 & 3.0 & 3.1 \\
\hline Investment budget & 4.8 & 1.0 & 1.5 & 3.0 & 5.1 & 4.8 & 3.3 & 1.8 \\
\hline A. EU flows & 4.6 & 1.0 & 1.4 & 2.8 & 4.9 & 4.6 & 3.1 & 1.6 \\
\hline B. Own revenues & 0.2 & 0.0 & 0.1 & 0.1 & 0.2 & 0.2 & 0.2 & 0.2 \\
\hline Gap filling measures & 0.0 & 0.0 & 0.0 & -0.1 & -0.1 & 1.7 & 2.8 & 3.9 \\
\hline Expenditure & 67.7 & 13.6 & 30.2 & 46.3 & 64.4 & 61.3 & 60.1 & 62.5 \\
\hline Ordinary spending & 59.7 & 12.8 & 27.6 & 42.5 & 57.7 & 56.1 & 56.0 & 58.4 \\
\hline Ordinary primary spending & 48.0 & 11.1 & 23.2 & 34.5 & 48.6 & 46.4 & 45.6 & 46.0 \\
\hline A. Remuneration and pensions & 20.4 & 4.6 & 9.8 & 14.9 & 20.7 & 20.4 & 20.4 & 20.3 \\
\hline B. Insurance and healthcare & 15.7 & 3.5 & 7.4 & 10.8 & 15.7 & 13.9 & 13.1 & 13.3 \\
\hline C. Operating and other expenditure & 6.9 & 1.4 & 3.0 & 4.7 & 6.7 & 6.4 & 6.3 & 6.4 \\
\hline D. Earmarked revenue & 4.0 & 1.2 & 2.3 & 3.1 & 4.3 & 4.4 & 4.6 & 4.8 \\
\hline E. Reserve & 0.4 & 0.3 & 0.6 & 0.9 & 1.2 & 1.2 & 1.2 & 1.3 \\
\hline F. EFSF commitment fee & 0.6 & 0.0 & 0.0 & 0.0 & 0.0 & 0.0 & 0.0 & 0.0 \\
\hline Interest & 11.7 & 1.7 & 4.4 & 8.0 & 9.1 & 9.7 & 10.3 & 12.4 \\
\hline Transfers to hospitals for the settlement of past debt & 0.4 & 0.1 & 0.1 & 0.1 & 0.4 & 0.4 & 0.4 & 0.4 \\
\hline Investment & 6.7 & 1.2 & 3.3 & 4.8 & 7.0 & 7.0 & 7.0 & 7.0 \\
\hline Spending on military procurement & 0.7 & 0.0 & 0.3 & 0.6 & 1.1 & 0.8 & 0.9 & 0.9 \\
\hline Guarantees on entities outside the general government & 0.2 & 0.0 & 0.1 & 0.1 & 0.5 & 0.3 & 0.2 & 0.2 \\
\hline Clearance of arrears & 0.0 & 0.0 & 0.0 & 0.0 & 0.0 & 0.0 & 0.0 & 0.0 \\
\hline Gap filling measures & 0.0 & -0.6 & -1.1 & -1.7 & -2.3 & -3.3 & -4.3 & -4.4 \\
\hline Balance & -15.3 & -2.1 & -7.9 & -10.8 & -13.1 & -10.9 & -9.5 & -10.6 \\
\hline Primary balance & -3.6 & -0.4 & -3.5 & -2.8 & -4.0 & -1.2 & 0.8 & 1.8 \\
\hline II. Balance local governments & 0.5 & 0.2 & 0.3 & 0.5 & 0.6 & 1.2 & 1.4 & 1.9 \\
\hline III. Balance social security funds & -0.4 & 2.9 & 3.8 & 3.5 & 3.8 & 3.4 & 3.2 & 4.0 \\
\hline IV. Balance of extra-budgetary funds (ETERPS) & 0.6 & 0.1 & 0.2 & 0.2 & 0.3 & 0.3 & 0.3 & 0.3 \\
\hline VIII. Balance of reclassified public enterprises & -0.8 & -0.1 & -0.3 & -0.4 & -0.5 & -0.5 & -0.6 & -0.6 \\
\hline X. Modified general government cash balance & -15.6 & 0.8 & -3.9 & -7.0 & -8.9 & -6.5 & -5.3 & -5.1 \\
\hline Modified general government primary cash balance & -3.8 & 2.5 & 0.5 & 1.0 & 0.2 & 3.2 & 5.1 & 7.3 \\
\hline Primary spending & 56.8 & 12.9 & 26.0 & 34.4 & 55.8 & 52.1 & 50.2 & 50.6 \\
\hline \multicolumn{9}{|l|}{ Memorandum items: } \\
\hline Floor on the modified general government primary cash balance & -3.8 & 1.5 & 0.5 & 1.0 & 0.2 & 3.2 & 5.1 & 7.3 \\
\hline Ceiling on primary spending & 56.8 & 14.9 & 26.0 & 38.8 & 55.8 & 52.1 & 50.2 & 50.6 \\
\hline
\end{tabular}

Sources: Ministry of Finance; and IMF staff projections. 
Table 5. Greece: Monetary Survey, 2007-12

\begin{tabular}{|c|c|c|c|c|c|c|c|c|c|c|c|c|}
\hline & \multirow[t]{3}{*}{2007} & \multirow[t]{3}{*}{2008} & \multirow[t]{3}{*}{2009} & \multirow[t]{3}{*}{2010} & \multicolumn{4}{|c|}{2011} & \multicolumn{4}{|c|}{2012} \\
\hline & & & & & \multirow[t]{2}{*}{ Mar-11 } & \multirow[t]{2}{*}{ Jun-11 } & \multirow[t]{2}{*}{ Sep-11 } & \multirow[t]{2}{*}{ Dec-11 } & \multirow[t]{2}{*}{ Mar-12 } & Jun-12 & Sep-12 & Dec-12 \\
\hline & & & & & & & & & & \multicolumn{3}{|c|}{ Proj. } \\
\hline & \multicolumn{12}{|c|}{ (Billions of euros) } \\
\hline \multicolumn{13}{|c|}{ Aggregated balance sheet of Monetary Financial Institutions (MFIs) } \\
\hline Total assets & 434.6 & 536.1 & 579.2 & 654.6 & 629.0 & 660.0 & 649.6 & 646.1 & 600.3 & 611.6 & 606.9 & 589.0 \\
\hline Cash (held by credit institutions) & 2.7 & 2.7 & 2.5 & 2.1 & 2.1 & 2.2 & 2.2 & 2.4 & 2.2 & 2.4 & 2.2 & 2.1 \\
\hline Claims on Bank of Greece & 7.2 & 7.9 & 8.2 & 10.6 & 9.9 & 10.8 & 6.0 & 5.1 & 1.8 & 3.5 & 3.4 & 3.4 \\
\hline Claims on other MFIs & 76.8 & 130.3 & 163.2 & 186.0 & 167.8 & 186.0 & 157.1 & 146.8 & 135.6 & 186.0 & 179.3 & 169.1 \\
\hline Claims (Loans) on non MFIs & 213.7 & 234.6 & 222.7 & 289.0 & 281.1 & 279.7 & 279.5 & 275.9 & 269.4 & 260.8 & 252.7 & 251.1 \\
\hline Domestic & 205.6 & 222.0 & 209.7 & 282.5 & 274.9 & 273.2 & 272.8 & 269.5 & 263.1 & 254.6 & 247.0 & 245.4 \\
\hline General government & 21.8 & 21.6 & 20.3 & 26.3 & 21.1 & 21.2 & 21.2 & 22.3 & 19.3 & 15.7 & 15.5 & 15.4 \\
\hline Other sectors $1 /$ & 183.7 & 200.4 & 189.3 & 256.2 & 253.8 & 252.0 & 251.5 & 247.2 & 243.8 & 238.9 & 231.5 & 230.0 \\
\hline Other countries & 8.2 & 12.6 & 13.0 & 6.5 & 6.2 & 6.4 & 6.7 & 6.4 & 6.3 & 6.2 & 5.6 & 5.8 \\
\hline Securities 2/ & 83.6 & 95.9 & 116.0 & 101.2 & 101.3 & 113.2 & 94.2 & 93.0 & 73.6 & 86.1 & 94.4 & 90.8 \\
\hline Other assets & 45.3 & 59.5 & 61.2 & 60.7 & 62.0 & 63.2 & 105.6 & 117.9 & 112.9 & 67.8 & 70.1 & 67.8 \\
\hline Fixed assets & 5.2 & 5.2 & 5.4 & 5.0 & 5.0 & 4.9 & 4.9 & 5.0 & 4.8 & 4.9 & 4.9 & 4.6 \\
\hline Total Liabilities & 434.6 & 536.1 & 579.2 & 654.6 & 629.0 & 660.0 & 649.6 & 646.1 & 600.3 & 611.6 & 606.9 & 589.0 \\
\hline Liabilities to Bank of Greece & 8.8 & 40.6 & 49.7 & 97.8 & 88.0 & 103.1 & 78.2 & 76.9 & 78.9 & 135.8 & 131.0 & 122.5 \\
\hline Liabilities to other MFIs & 88.0 & 121.9 & 149.4 & 164.7 & 148.8 & 173.6 & 163.4 & 155.4 & 149.3 & 147.8 & 144.9 & 136.1 \\
\hline Deposits and repos of non MFIs & 249.7 & 281.7 & 280.1 & 282.5 & 279.5 & 251.9 & 251.5 & 237.5 & 226.8 & 211.2 & 214.8 & 220.4 \\
\hline Domestic & 206.1 & 237.4 & 246.7 & 225.1 & 221.9 & 199.7 & 195.6 & 187.7 & 179.6 & 163.5 & 167.5 & 173.6 \\
\hline Other countries & 43.6 & 44.3 & 33.4 & 57.4 & 57.6 & 52.2 & 55.8 & 49.8 & 47.3 & 47.7 & 47.3 & 46.8 \\
\hline Capital and reserves & 30.6 & 30.0 & 41.6 & 46.7 & 47.6 & 48.5 & 48.9 & 54.8 & 24.0 & 42.6 & 50.1 & 50.2 \\
\hline Banknotes and coins in circulation & 16.9 & 19.0 & 21.6 & 22.5 & 22.1 & 22.6 & 22.9 & 23.7 & 23.2 & 23.8 & 23.8 & 23.4 \\
\hline Other liabilities & 40.5 & 42.8 & 36.7 & 40.3 & 43.0 & 60.2 & 84.7 & 97.8 & 97.9 & 50.5 & 42.3 & 36.2 \\
\hline \multicolumn{13}{|l|}{ Money and credit } \\
\hline Broad money & 219.9 & 251.4 & 261.1 & 232.9 & 223.2 & 212.8 & 207.7 & 199.2 & 190.1 & 178.3 & 181.7 & 183.1 \\
\hline Credit to the private sector $3 / 4$ / & 215.1 & 249.3 & 249.3 & 257.5 & 255.0 & 253.1 & 252.6 & 248.1 & 244.7 & 239.8 & 231.4 & 230.0 \\
\hline \multirow[t]{2}{*}{ Credit to government 3 / } & 36.6 & 37.1 & 51.0 & 63.0 & 58.8 & 62.5 & 58.0 & 59.1 & 34.8 & 26.9 & 29.6 & 26.9 \\
\hline & \multicolumn{12}{|c|}{ (Annual percent change) $5 /$} \\
\hline Broad money & 13.8 & 12.4 & 4.1 & -11.2 & -9.8 & -10.2 & -12.0 & -14.6 & -15.1 & -17.9 & -13.2 & -8.1 \\
\hline Domestic private sector deposits & 14.3 & 13.3 & 4.9 & -12.4 & -12.3 & -12.0 & -14.1 & -17.0 & -17.4 & -20.0 & -15.5 & -10.2 \\
\hline Credit to the private sector $3 / 4$ / & 21.5 & 15.9 & 4.2 & 0.0 & -0.4 & -1.2 & -2.2 & -3.1 & -4.0 & -4.3 & -4.5 & -7.2 \\
\hline \multirow[t]{2}{*}{ Credit to government 3 / } & -8.8 & 1.3 & 19.4 & 28.3 & 8.7 & 8.9 & 3.3 & 2.0 & 3.6 & -3.1 & 0.0 & -54.6 \\
\hline & \multicolumn{12}{|c|}{ (Percent of GDP) } \\
\hline Broad money & 98.6 & 107.8 & 113.0 & 104.8 & 102.4 & 99.3 & 97.9 & 95.5 & 92.5 & 88.4 & 92.2 & 93.9 \\
\hline Domestic deposits & 92.4 & 101.8 & 106.8 & 101.3 & 101.8 & 93.2 & 92.2 & 90.0 & 87.4 & 81.1 & 85.0 & 89.1 \\
\hline Credit to the private sector 4 / & 96.4 & 106.9 & 107.9 & 115.9 & 117.0 & 118.1 & 119.1 & 119.0 & 119.1 & 118.9 & 117.5 & 118.0 \\
\hline Credit to government & 16.4 & 15.9 & 22.1 & 28.4 & 27.0 & 29.1 & 27.3 & 28.4 & 16.9 & 13.3 & 15.0 & 13.8 \\
\hline Memorandum items: & \multicolumn{12}{|c|}{ (Percent) } \\
\hline Capital to assets & 5.8 & 4.7 & 6.4 & 5.8 & 5.7 & 5.9 & 5.9 & 6.9 & -1.0 & 3.4 & 4.8 & 4.7 \\
\hline Loans to customer deposits & 84.8 & 83.2 & 79.2 & 103.5 & 102.1 & 112.8 & 111.7 & 117.0 & 118.7 & 124.2 & 117.6 & 113.3 \\
\hline Velocity & 1.0 & 0.9 & 0.9 & 1.0 & 1.0 & 1.0 & 1.0 & 1.0 & 1.1 & 1.1 & 1.1 & 1.1 \\
\hline
\end{tabular}

Sources: Bank of Greece; and IMF staff estimates and projections.

1/ As of June 2010, securitised assets are no longer derecognised from the balance sheet of banks that have adopted the International Accounting Standards. The counterpart of these assets is recorded on the liabilities side as deposit liabilities to non-euro area residents.

2/ Holdings of securities other than shares and derivatives.

3/ Projections do not take into account write-offs, valuation changes, or reclassifications.

4/ Credit to domestic non-MFI residents by domestic MFIs excluding the Bank of Greece, including securitized loans and corporate bonds.

5/ Growth rates are calculated from differences in outstanding amounts adjusted for revaluations, exchange rate valuation differences, reclassifications and any other changes which do not arise from transactions. 
Table 6. Greece: Core Set of Financial Soundness Indicators for Deposit-Taking Institutions, 2008-12

\begin{tabular}{|c|c|c|c|c|c|c|c|c|c|c|c|}
\hline & \multirow[t]{2}{*}{2008} & \multirow[t]{2}{*}{2009} & \multicolumn{4}{|c|}{2010} & \multicolumn{4}{|c|}{2011} & \multirow{2}{*}{2012} \\
\hline & & & Mar. & Jun. & Sep. & Dec. & Mar. & Jun. & Sep. & Dec. & \\
\hline \multicolumn{12}{|l|}{ Core set } \\
\hline Regulatory capital to risk-weighted assets $1 /$ & 9.6 & 11.9 & 11.7 & 11.2 & 11.4 & 12.3 & 12.3 & 10.6 & 10.1 & 7.0 & 5.7 \\
\hline Excluding bridge capital & $\ldots$ & $\ldots$ & $\ldots$ & $\ldots$ & $\ldots$ & $\ldots$ & $\ldots$ & $\ldots$ & $\ldots$ & -1.7 & -2.9 \\
\hline Regulatory tier I capital to risk-weighted assets 1 / & 8.1 & 10.9 & 10.6 & 10.1 & 10.1 & 11.2 & 11.1 & 9.6 & 9.2 & 5.9 & 4.8 \\
\hline Excluding bridge capital & $\ldots$ & $\ldots$ & $\ldots$ & $\ldots$ & $\ldots$ & $\ldots$ & $\ldots$ & $\ldots$ & $\ldots$ & -2.6 & -3.6 \\
\hline Nonperforming loans net of provisions to capital & 24.7 & 37.9 & 40.6 & 45.4 & 49.9 & 46.9 & 51.2 & 66.3 & 78.6 & $\ldots$ & $\ldots$ \\
\hline Nonperforming loans to total gross loans & 5.1 & 7.8 & 8.2 & 9.0 & 10.0 & 10.5 & 11.5 & 12.8 & 14.7 & 16.0 & 18.7 \\
\hline Bank provisions to nonperforming loans & 49.4 & 42.0 & $\ldots$ & $\ldots$ & $\ldots$ & 46.2 & $\ldots$ & $\ldots$ & $\ldots$ & 62.9 & 57.1 \\
\hline Return on assets (after taxes) 2/ & 0.2 & -0.1 & -0.6 & -0.6 & -0.5 & -0.6 & -0.3 & -1.7 & -2.1 & $\ldots$ & $\ldots$ \\
\hline Return on equity (after taxes) 2/ & 3.2 & -1.7 & -8.7 & -9.7 & -8.4 & -8.8 & -3.9 & -27.3 & -34.2 & $\ldots$ & $\ldots$ \\
\hline Interest margin to gross income $2 /$ & 83.2 & 75.8 & 98.1 & 97.4 & 93.9 & 91.0 & 82.5 & 87.4 & 91.7 & 93.3 & 98.8 \\
\hline Non-interest expenses to gross income 2/ & 59.5 & 57.4 & 65.8 & 66.0 & 63.9 & 62.1 & 54.1 & 56.9 & 58.6 & 62.0 & 68.3 \\
\hline Liquid assets to total assets 3 / & 38.7 & 45.2 & 45.9 & 39.6 & 36.5 & 35.2 & 34.5 & 35.2 & 33.2 & 32.3 & 27.3 \\
\hline Liquid assets to short-term liabilities 3 / & 50.0 & 56.9 & 57.4 & 55.0 & 49.6 & 46.9 & 46.4 & 48.2 & 50.2 & 43.1 & 34.0 \\
\hline Net open position in foreign exchange to capital $1 / 4$ / & 7.8 & 11.6 & 10.8 & 10.7 & 8.5 & 11.3 & 12.0 & 15.4 & 14.5 & $\ldots$ & $\ldots$ \\
\hline \multicolumn{12}{|l|}{ Encouraged set } \\
\hline $\begin{array}{l}\text { Spread between reference lending and deposit rates (end-of- } \\
\text { period, basis points) } 5 \text { / }\end{array}$ & 5.8 & 5.2 & $\ldots$ & $\ldots$ & $\ldots$ & 6.4 & $\ldots$ & $\ldots$ & $\ldots$ & 7.5 & 7.4 \\
\hline Customer deposits to total (noninterbank) loans $3 /$ & 127.4 & 125.7 & $\ldots$ & $\ldots$ & $\ldots$ & 96.6 & $\ldots$ & $\ldots$ & $\ldots$ & 85.5 & 84.2 \\
\hline $\begin{array}{l}\text { Foreign currency-denominated liabilities to total liabilities } 3 \text { / } \\
\text { Market liquidity }\end{array}$ & 12.7 & 10.8 & $\ldots$ & $\ldots$ & $\ldots$ & 9.0 & $\ldots$ & $\ldots$ & $\ldots$ & 7.7 & 7.1 \\
\hline $\begin{array}{l}\text { Average bid-ask spread in the securities market (basis } \\
\text { points) }\end{array}$ & 55.9 & 60.6 & $\ldots$ & $\ldots$ & $\ldots$ & 126.0 & $\ldots$ & $\ldots$ & $\ldots$ & 87.2 & 177.0 \\
\hline \multicolumn{12}{|l|}{ Households } \\
\hline Household debt to GDP & 50.3 & 52.1 & $\ldots$ & $\ldots$ & $\ldots$ & 60.0 & $\ldots$ & $\ldots$ & $\ldots$ & 61.3 & 63.8 \\
\hline \multicolumn{12}{|l|}{ Real estate markets } \\
\hline Residential real estate loans to total loans $3 /$ & 20.7 & 20.3 & $\ldots$ & $\ldots$ & $\ldots$ & 21.1 & $\ldots$ & $\ldots$ & $\ldots$ & 22.8 & 24.2 \\
\hline \multicolumn{12}{|l|}{ Memorandum items: } \\
\hline \multicolumn{12}{|l|}{ Assets (billions of euros) } \\
\hline Banks & 412.1 & 440.3 & $\ldots$ & $\ldots$ & $\ldots$ & 465.8 & $\ldots$ & $\ldots$ & $\ldots$ & 850.3 & 773.1 \\
\hline Branches of foreign banks & 39.4 & 38.3 & $\ldots$ & $\ldots$ & $\ldots$ & 36.9 & $\ldots$ & $\ldots$ & $\ldots$ & 52.0 & 47.6 \\
\hline General insurance companies 6/ & 15.1 & 15.5 & $\ldots$ & $\ldots$ & $\ldots$ & 15.7 & $\ldots$ & $\ldots$ & $\ldots$ & 15.8 & 15.6 \\
\hline Other credit institutions & 10.5 & 11.6 & $\ldots$ & $\ldots$ & $\ldots$ & 11.4 & $\ldots$ & $\ldots$ & $\ldots$ & 11.5 & 10.6 \\
\hline \multicolumn{12}{|l|}{ Deposits (billions of euros) } \\
\hline Banks & 251.5 & 248.6 & $\ldots$ & $\ldots$ & $\ldots$ & 220.3 & $\ldots$ & $\ldots$ & $\ldots$ & 71.5 & 69.1 \\
\hline Branches of foreign banks & 21.2 & 21.9 & $\ldots$ & $\ldots$ & $\ldots$ & 18.7 & $\ldots$ & $\ldots$ & $\ldots$ & 20.7 & 19.9 \\
\hline
\end{tabular}

Source: Bank of Greece.

1/ Data on a consolidated basis. For end-2011 and 2012Q1, C.A.R. ratios are affected by the PSI and include only the first tranche of $€ 18$ billion HFSF recapitalization. In addittion, C.A.R. ratios are affected by the negative supervisory own funds of two banks (ATEbank and TT Hellenic Post Bank).

2/ From 2004 in accordance with IFRS.

3/ On an aggregate resident-based approach (i.e. commercial banks, cooperative banks, and foreign branches).

4/ Based on revised figures from 2002 onwards.

5/ Spread between rate on credit lines and savings deposit rate.

6/ There are no specialised life insurance companies in Greece. General insurance companies offer general insurance and life insurance products. 


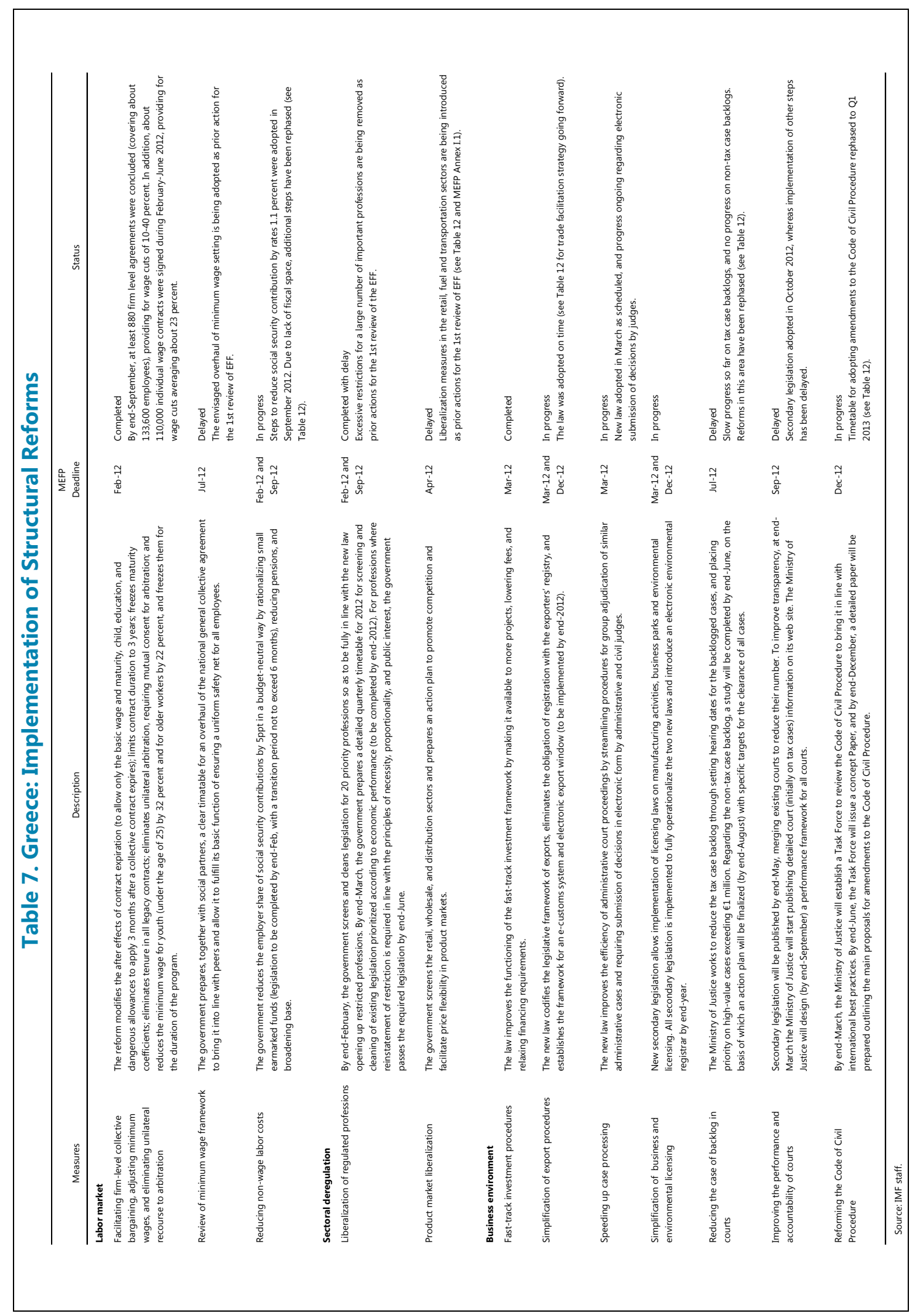




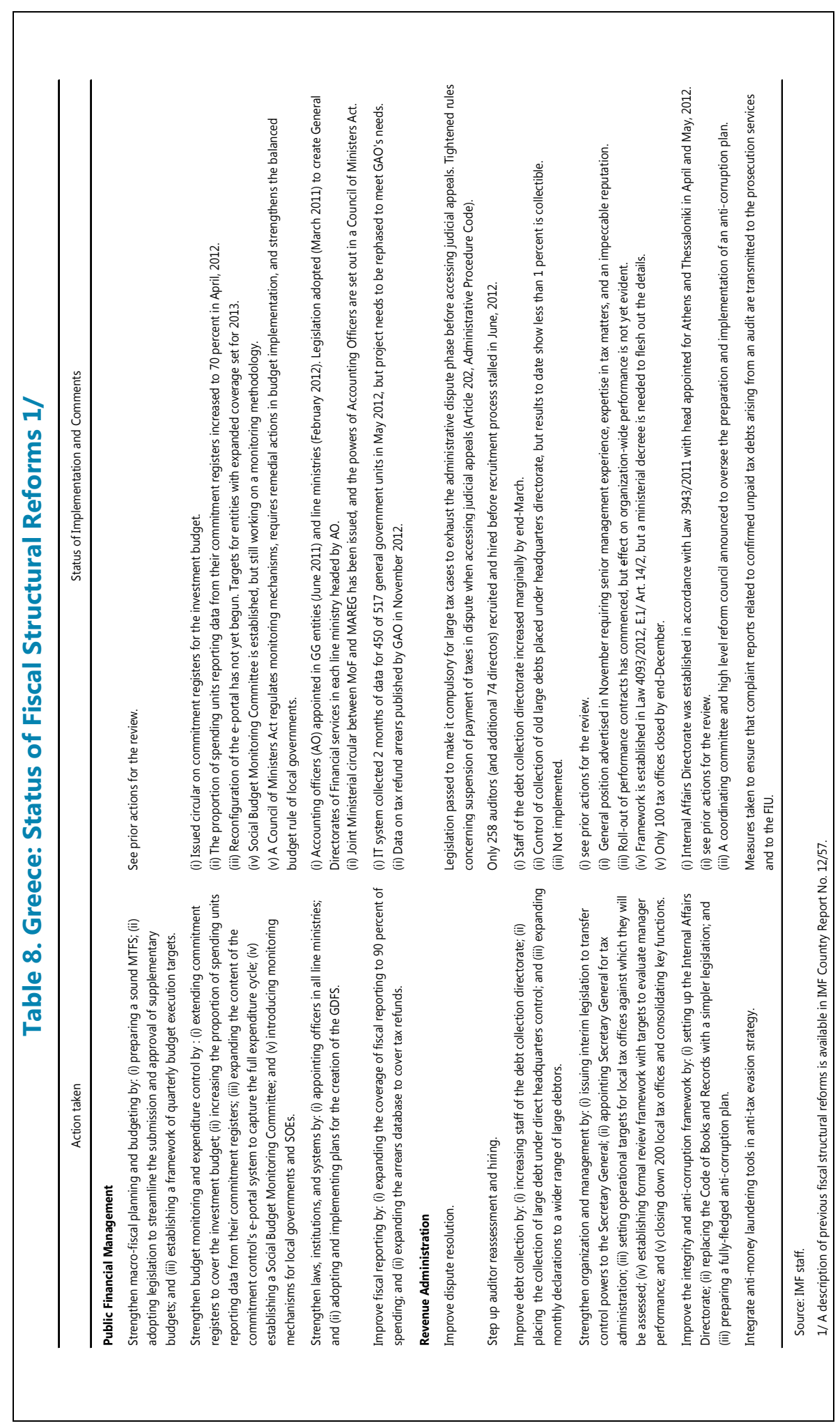

INTERNATIONAL MONETARY FUND 
Table 9. Greece: Medium-Term Macro Framework, 2012-20

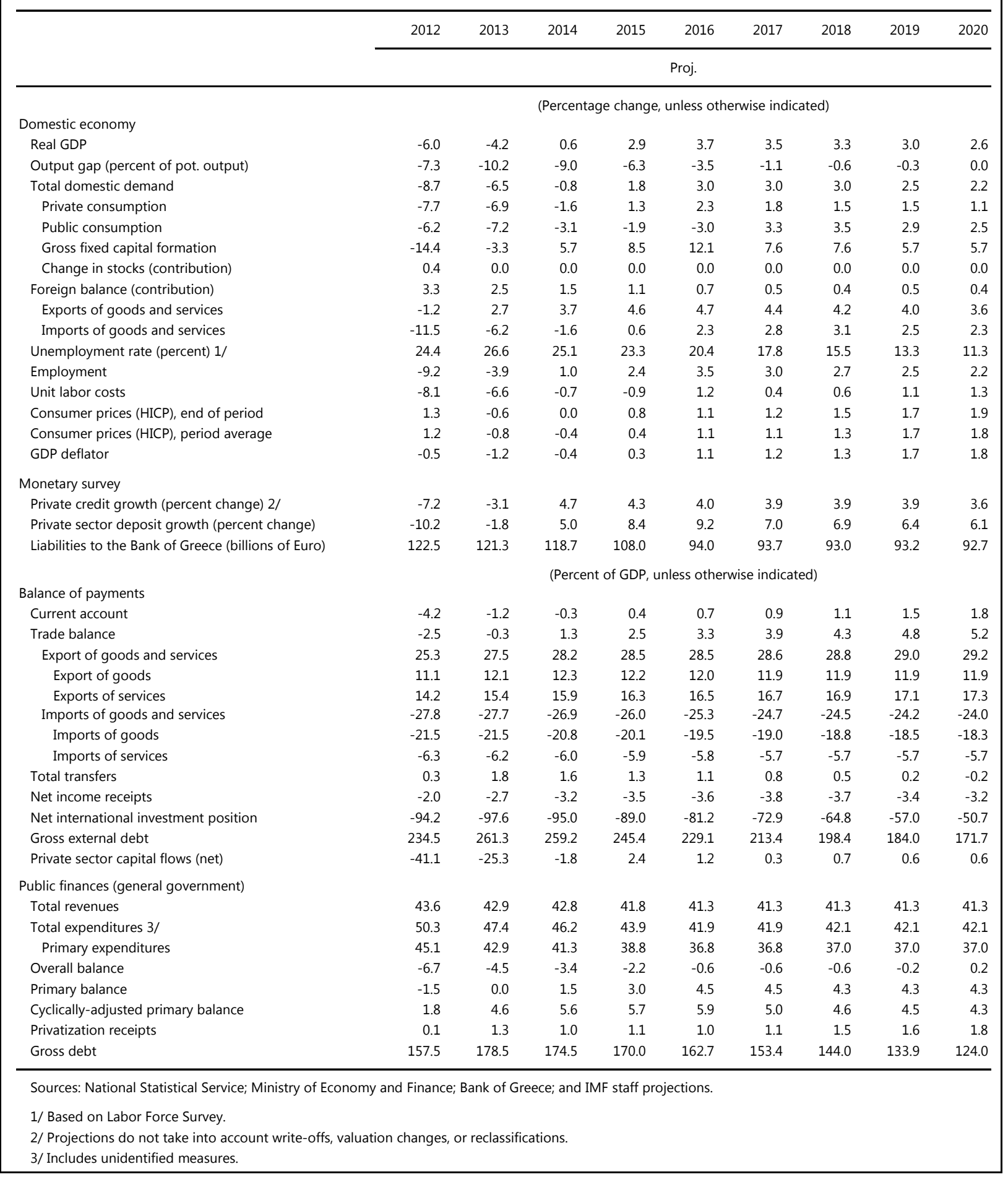


Table 10. Greece: Summary of Balance of Payments, 2010-17

\begin{tabular}{|c|c|c|c|c|c|c|c|c|}
\hline & 2010 & 2011 & 2012 & 2013 & 2014 & 2015 & 2016 & 2017 \\
\hline & & & \multicolumn{6}{|c|}{ Proj. } \\
\hline & \multicolumn{8}{|c|}{ (Billions of euro) } \\
\hline Current account balance & -22.5 & -20.6 & -8.1 & -2.3 & -0.5 & 0.8 & 1.4 & 1.8 \\
\hline Balance of goods and services & -15.0 & -12.6 & -4.8 & -0.5 & 2.4 & 4.8 & 6.5 & 8.2 \\
\hline Goods balance & -28.3 & -27.2 & -20.3 & -17.5 & -15.9 & -15.1 & -14.9 & -14.9 \\
\hline Exports & 17.1 & 20.2 & 21.7 & 22.2 & 22.7 & 23.3 & 24.0 & 24.9 \\
\hline Imports & 45.4 & 47.5 & 41.9 & 39.7 & 38.5 & 38.4 & 38.9 & 39.8 \\
\hline Services balance & 13.2 & 14.6 & 15.5 & 17.0 & 18.3 & 19.9 & 21.4 & 23.0 \\
\hline Credit & 28.5 & 28.6 & 27.7 & 28.4 & 29.5 & 31.2 & 33.1 & 35.1 \\
\hline Debit & 15.2 & 14.0 & 12.3 & 11.5 & 11.2 & 11.3 & 11.6 & 12.0 \\
\hline Income balance & -7.7 & -8.6 & -3.8 & -5.0 & -5.9 & -6.6 & -7.3 & -8.0 \\
\hline Credit & 4.0 & 3.3 & 3.3 & 4.2 & 4.9 & 6.0 & 7.0 & 7.9 \\
\hline Debit & 11.7 & 11.9 & 7.1 & 9.2 & 10.9 & 12.6 & 14.3 & 15.9 \\
\hline Current transfers (net) & 0.2 & 0.6 & 0.5 & 3.3 & 3.0 & 2.6 & 2.1 & 1.7 \\
\hline Capital and financial account balance & -8.6 & -21.0 & -101.8 & -37.0 & -17.2 & -18.8 & -10.3 & -7.3 \\
\hline Capital account balance & 2.1 & 2.7 & 3.9 & 3.9 & 3.5 & 3.3 & 3.3 & 3.3 \\
\hline Financial account & -10.6 & -23.7 & -105.6 & -40.9 & -20.7 & -22.1 & -13.6 & -10.6 \\
\hline Direct investment & -0.9 & -0.5 & 1.1 & 1.7 & 1.8 & 1.9 & 1.8 & 2.1 \\
\hline Portfolio investment & -20.9 & -19.8 & -12.9 & -29.7 & -9.0 & -5.2 & -2.1 & -3.5 \\
\hline Of which: government & -26.8 & -23.3 & -10.6 & -9.0 & -9.1 & -6.1 & -2.7 & -4.7 \\
\hline Other investment (excl. program financing) & 11.0 & -3.4 & -93.8 & -12.8 & -13.4 & -18.8 & -13.3 & -9.2 \\
\hline Reserve assets (increase $=-$ ) & 0.1 & 0.0 & 0.0 & 0.0 & 0.0 & 0.0 & 0.0 & 0.0 \\
\hline Net errors and omissions & -0.4 & 0.1 & 0.0 & 0.0 & 0.0 & 0.0 & 0.0 & 0.0 \\
\hline Program financing & 31.5 & 41.5 & 109.9 & 39.2 & 17.6 & 10.9 & 6.4 & 5.4 \\
\hline \multirow[t]{2}{*}{ Unidentified financing } & 0.0 & 0.0 & 0.0 & 0.0 & 0.0 & 7.1 & 2.5 & 0.0 \\
\hline & \multicolumn{8}{|c|}{ (Percent of GDP) } \\
\hline Current account balance & -10.1 & -9.9 & -4.2 & -1.2 & -0.3 & 0.4 & 0.7 & 0.9 \\
\hline Balance on goods and services & -6.8 & -6.0 & -2.5 & -0.3 & 1.3 & 2.5 & 3.3 & 3.9 \\
\hline Goods balance & -12.7 & -13.1 & -10.4 & -9.5 & -8.6 & -7.9 & -7.4 & -7.1 \\
\hline Services balance & 6.0 & 7.0 & 7.9 & 9.2 & 9.9 & 10.4 & 10.7 & 11.0 \\
\hline Income balance & -3.5 & -4.1 & -2.0 & -2.7 & -3.2 & -3.5 & -3.6 & -3.8 \\
\hline Current transfers & 0.1 & 0.3 & 0.3 & 1.8 & 1.6 & 1.3 & 1.1 & 0.8 \\
\hline Capital and financial account balance & -3.9 & -10.1 & -52.2 & -20.0 & -9.3 & -9.8 & -5.1 & -3.5 \\
\hline Capital account balance & 0.9 & 1.3 & 2.0 & 2.1 & 1.9 & 1.7 & 1.7 & 1.6 \\
\hline Financial account & -4.8 & -11.3 & -54.2 & -22.1 & -11.2 & -11.6 & -6.8 & -5.1 \\
\hline Direct investment & -0.4 & -0.2 & 0.6 & 0.9 & 0.9 & 1.0 & 0.9 & 1.0 \\
\hline Portfolio investment & -9.4 & -9.5 & -6.6 & -16.1 & -4.9 & -2.7 & -1.1 & -1.7 \\
\hline Of which: government & -12.1 & -11.2 & -5.4 & -4.9 & -4.9 & -3.2 & -1.4 & -2.2 \\
\hline Other investment & 5.0 & -1.7 & -48.1 & -7.0 & -7.3 & -9.8 & -6.7 & -4.4 \\
\hline Reserve assets (increase $=-$ ) & 0.0 & 0.0 & 0.0 & 0.0 & 0.0 & 0.0 & 0.0 & 0.0 \\
\hline Net errors and omissions & -0.2 & 0.1 & 0.0 & 0.0 & 0.0 & 0.0 & 0.0 & 0.0 \\
\hline Program financing & 14.2 & 19.9 & 56.4 & 21.3 & 9.5 & 5.7 & 3.2 & 2.6 \\
\hline Unidentified official financing / market access & 0.0 & 0.0 & 0.0 & 0.0 & 0.0 & 3.7 & 1.3 & 0.0 \\
\hline Gross external debt & 213.1 & 225.4 & 234.5 & 261.3 & 259.2 & 245.4 & 229.1 & 213.4 \\
\hline Public sector & 111.7 & 123.3 & 131.7 & 154.6 & 154.9 & 151.7 & 146.3 & 139.7 \\
\hline Private sector & 101.4 & 102.1 & 102.8 & 106.7 & 104.3 & 93.6 & 82.8 & 73.8 \\
\hline \multicolumn{9}{|l|}{ Memorandum item: } \\
\hline Current account balance in cash terms & -10.1 & -9.9 & -4.2 & -0.6 & 0.8 & 2.4 & 3.0 & 3.4 \\
\hline
\end{tabular}


Table 11. Greece: Monetary Financial Institutions (excl. BoG)—Uses and Sources of Funds, 2006-16

\begin{tabular}{|c|c|c|c|c|c|c|c|c|c|c|c|}
\hline & 2006 & 2007 & 2008 & 2009 & 2010 & 2011 & 2012 & 2013 & 2014 & 2015 & 2016 \\
\hline & & & & & & & \multicolumn{5}{|c|}{ Proj. } \\
\hline & \multicolumn{11}{|c|}{ (Billions of euros) } \\
\hline Assets & 321.0 & 391.3 & 464.5 & 491.9 & 515.3 & 476.9 & 433.7 & 447.0 & 453.5 & 465.7 & 481.3 \\
\hline Cash & 2.6 & 2.7 & 2.7 & 2.5 & 2.1 & 2.4 & 2.1 & 2.0 & 2.0 & 2.1 & 2.2 \\
\hline Claims on other MFIs & 43.6 & 66.3 & 88.5 & 112.2 & 87.0 & 68.7 & 46.5 & 45.4 & 43.8 & 43.7 & 44.2 \\
\hline Claims on non-MFIs & 227.5 & 264.8 & 301.1 & 309.3 & 357.8 & 339.2 & 315.6 & 330.5 & 338.6 & 348.6 & 360.2 \\
\hline General government & 35.7 & 31.5 & 31.6 & 41.8 & 63.0 & 59.1 & 26.9 & 26.4 & 26.4 & 27.4 & 28.7 \\
\hline Private sector $1 /$ & 170.7 & 202.4 & 221.3 & 211.7 & 256.9 & 247.6 & 230.6 & 223.4 & 233.9 & 244.0 & 253.7 \\
\hline Corporate & 85.6 & 102.2 & 124.1 & 123.8 & 116.5 & 113.0 & 103.1 & 101.9 & 109.2 & 116.4 & 123.8 \\
\hline Households & 85.1 & 100.2 & 97.2 & 87.9 & 140.4 & 134.6 & 127.5 & 121.5 & 124.7 & 127.5 & 130.0 \\
\hline Other countries & 21.1 & 31.0 & 48.1 & 55.7 & 37.9 & 32.4 & 58.1 & 80.8 & 78.2 & 77.3 & 77.8 \\
\hline Other assets & 47.4 & 57.6 & 72.2 & 67.9 & 68.4 & 66.6 & 69.5 & 69.1 & 69.0 & 71.4 & 74.8 \\
\hline Liabilities & 321.0 & 391.3 & 464.5 & 491.9 & 515.0 & 476.9 & 433.7 & 447.0 & 453.5 & 465.7 & 481.3 \\
\hline Liabilities to other MFIs & 45.1 & 69.1 & 77.8 & 91.5 & 66.3 & 44.5 & 32.3 & 30.8 & 30.9 & 31.8 & 33.3 \\
\hline Deposits of non-MFIs & 211.1 & 248.5 & 280.2 & 278.8 & 280.2 & 232.3 & 215.6 & 208.8 & 215.6 & 232.3 & 253.4 \\
\hline Central government & 3.7 & 4.0 & 4.2 & 3.1 & 9.0 & 4.1 & 8.9 & 7.5 & 6.4 & 6.4 & 7.2 \\
\hline Private sector & 177.2 & 201.0 & 231.6 & 242.4 & 213.9 & 178.7 & 160.4 & 157.5 & 165.3 & 179.2 & 195.8 \\
\hline Other countries 2/ & 30.1 & 43.6 & 44.3 & 33.4 & 57.4 & 49.5 & 46.3 & 43.8 & 43.9 & 46.8 & 50.5 \\
\hline Other liabilities & 35.2 & 36.0 & 37.9 & 32.6 & 26.3 & 70.1 & 15.8 & 14.9 & 15.0 & 15.5 & 16.2 \\
\hline Capital and reserves & 24.8 & 28.9 & 28.0 & 39.3 & 44.4 & 53.1 & 47.5 & 71.1 & 73.4 & 78.1 & 84.4 \\
\hline \multirow[t]{2}{*}{ Eurosystem liquidity support } & 4.9 & 8.8 & 40.6 & 49.7 & 97.8 & 76.9 & 122.5 & 121.3 & 118.7 & 108.0 & 94.0 \\
\hline & \multicolumn{11}{|c|}{ (Percent of GDP) } \\
\hline Assets & 153.9 & 175.4 & 199.2 & 212.9 & 232.0 & 228.7 & 222.4 & 242.3 & 245.2 & 243.9 & 240.4 \\
\hline Cash & 1.2 & 1.2 & 1.2 & 1.1 & 1.0 & 1.1 & 1.1 & 1.1 & 1.1 & 1.1 & 1.1 \\
\hline Claims on other MFIs & 20.9 & 29.7 & 38.0 & 48.6 & 39.2 & 33.0 & 23.9 & 24.6 & 23.7 & 22.9 & 22.1 \\
\hline Claims on non-MFIs & 109.0 & 118.6 & 129.1 & 133.8 & 161.1 & 162.7 & 161.9 & 179.1 & 183.1 & 182.6 & 179.9 \\
\hline General government & 17.1 & 14.1 & 13.6 & 18.1 & 28.4 & 28.4 & 13.8 & 14.3 & 14.3 & 14.3 & 14.3 \\
\hline Private sector $1 /$ & 81.8 & 90.7 & 94.9 & 91.6 & 115.6 & 118.8 & 118.3 & 121.1 & 126.5 & 127.7 & 126.7 \\
\hline Corporate & 41.0 & 45.8 & 53.2 & 53.6 & 52.4 & 54.2 & 52.9 & 55.2 & 59.0 & 61.0 & 61.8 \\
\hline Households & 40.8 & 44.9 & 41.7 & 38.0 & 63.2 & 64.5 & 65.4 & 65.8 & 67.4 & 66.8 & 64.9 \\
\hline Other countries & 10.1 & 13.9 & 20.6 & 24.1 & 17.1 & 15.6 & 29.8 & 43.8 & 42.3 & 40.5 & 38.8 \\
\hline Other assets & 22.7 & 25.8 & 31.0 & 29.4 & 30.8 & 31.9 & 35.6 & 37.5 & 37.3 & 37.4 & 37.4 \\
\hline Liabilities & 153.9 & 175.4 & 199.2 & 212.9 & 231.8 & 228.7 & 222.4 & 242.3 & 245.2 & 243.9 & 240.4 \\
\hline Liabilities to other MFIs & 21.6 & 31.0 & 33.4 & 39.6 & 29.8 & 21.4 & 16.6 & 16.7 & 16.7 & 16.7 & 16.6 \\
\hline Deposits of non-MFIs & 101.2 & 111.4 & 120.1 & 120.7 & 126.1 & 111.4 & 110.6 & 113.2 & 116.6 & 121.7 & 126.6 \\
\hline Central government & 1.8 & 1.8 & 1.8 & 1.3 & 4.0 & 2.0 & 4.6 & 4.1 & 3.5 & 3.4 & 3.6 \\
\hline Private sector & 84.9 & 90.1 & 99.3 & 104.9 & 96.3 & 85.7 & 82.3 & 85.3 & 89.4 & 93.8 & 97.8 \\
\hline Other countries 2/ & 14.5 & 19.5 & 19.0 & 14.4 & 25.8 & 23.7 & 23.7 & 23.8 & 23.8 & 24.5 & 25.2 \\
\hline Other liabilities & 16.9 & 16.1 & 16.3 & 14.1 & 11.8 & 33.6 & 8.1 & 8.1 & 8.1 & 8.1 & 8.1 \\
\hline Capital and reserves & 11.9 & 12.9 & 12.0 & 17.0 & 20.0 & 25.4 & 24.4 & 38.5 & 39.7 & 40.9 & 42.1 \\
\hline Eurosystem liquidity support & 2.3 & 3.9 & 17.4 & 21.5 & 44.0 & 36.9 & 62.8 & 65.8 & 64.2 & 56.6 & 47.0 \\
\hline \multicolumn{12}{|l|}{ Memorandum items: } \\
\hline Domestic private sector deposit growth (percent) & 10.9 & 14.3 & 13.3 & 4.9 & -12.4 & -17.0 & -10.2 & -1.8 & 5.0 & 8.4 & 9.2 \\
\hline Private sector credit growth (percent) 3/ & 21.1 & 21.5 & 15.9 & 4.2 & 0.0 & -3.1 & -7.2 & -3.1 & 4.7 & 4.3 & 4.0 \\
\hline Eurosystem liquidity support (percent of total assets) & 1.5 & 2.2 & 8.7 & 10.1 & 19.0 & 16.1 & 28.2 & 27.1 & 26.2 & 23.2 & 19.5 \\
\hline
\end{tabular}

Sources: Bank of Greece; and IMF staff estimates and projections.

1/ As of June 2010, securitised assets are no longer derecognised from the balance sheet of banks that have adopted the International Accounting Standards. The counterpart of these assets is recorded on the liabilities side as deposit liabilities to non-euro area residents.

2/ June 2010 reclassification related to liabilities associated with assets disposed of in a securitisation but still recognised on the statistical balance sheet.

3/ Projections do not take into account write-offs, valuation changes, or reclassifications. 


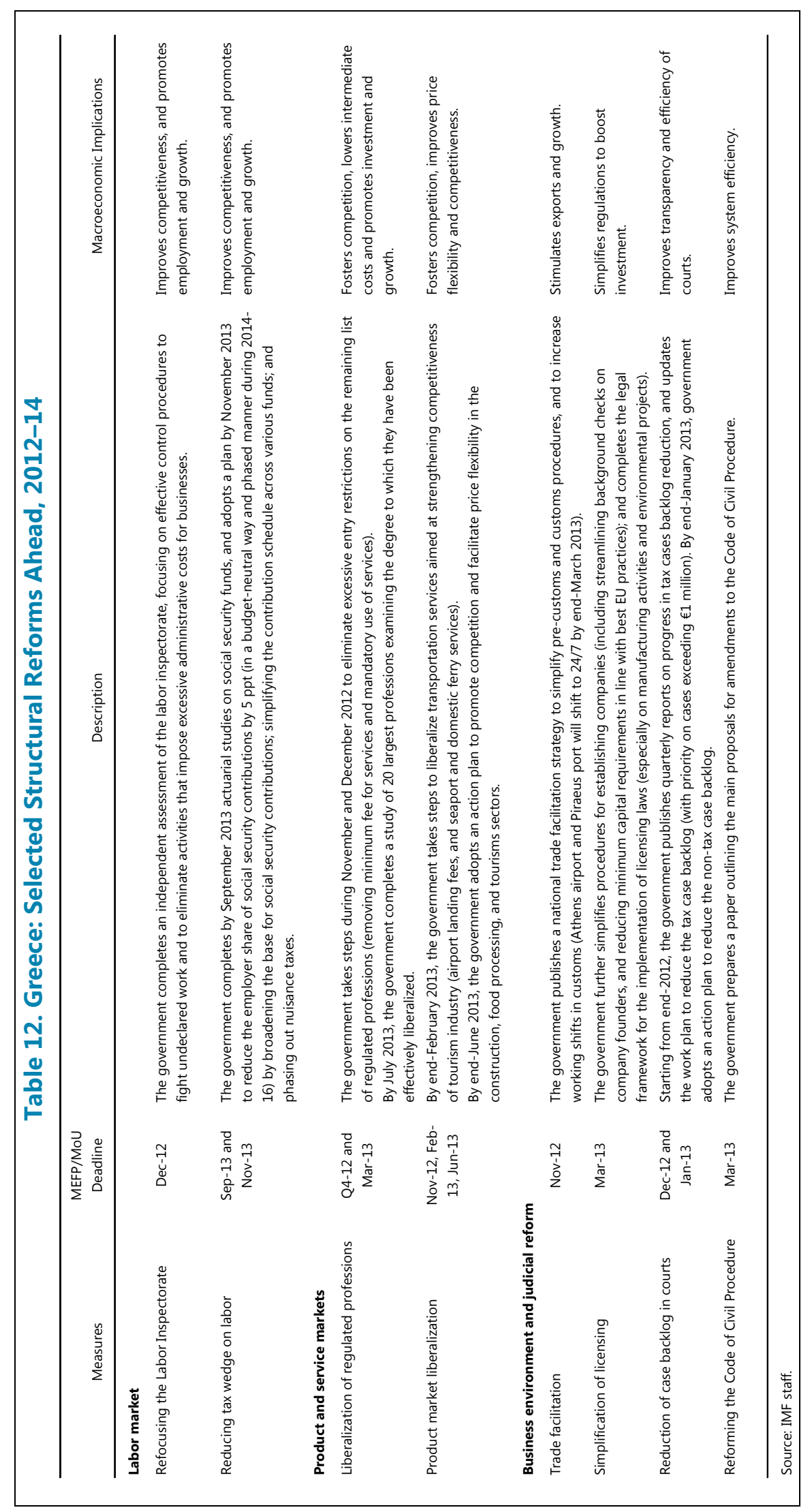




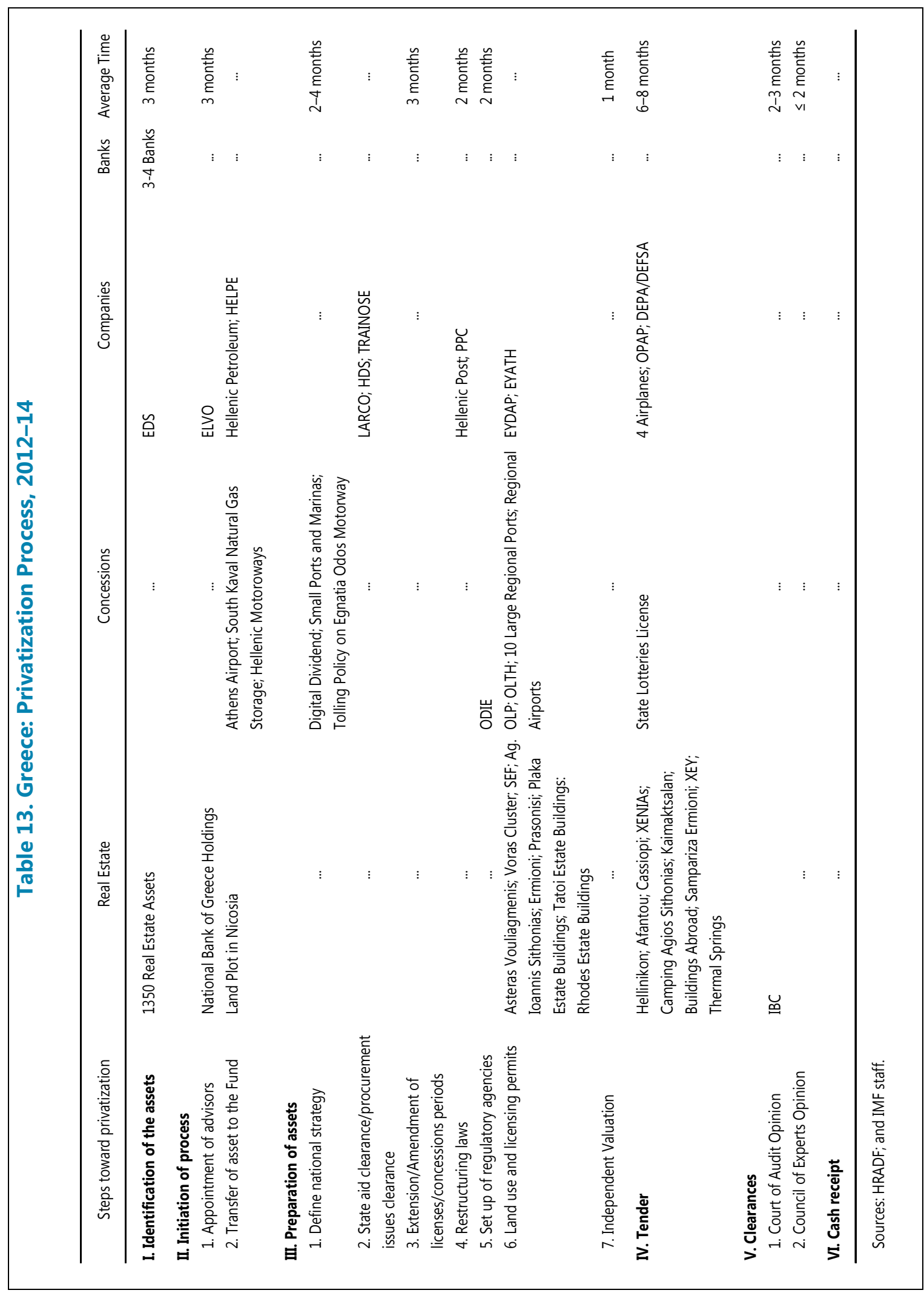


Table 14. Greece: General Government Operations, 2012-16

\begin{tabular}{|c|c|c|c|c|c|c|}
\hline & \multicolumn{2}{|c|}{2012} & 2013 & 2014 & 2015 & 2016 \\
\hline & Prog. & Proj. & \multicolumn{4}{|c|}{ Proj. } \\
\hline & \multicolumn{6}{|c|}{ (Billions of euros) } \\
\hline Revenue & 86.0 & 85.0 & 79.1 & 79.2 & 79.8 & 82.7 \\
\hline Taxes on production, and imports & 25.5 & 25.1 & 23.9 & 23.7 & 24.3 & 25.8 \\
\hline Taxes on income and property & 19.3 & 19.8 & 17.5 & 18.3 & 18.7 & 19.2 \\
\hline Social contributions & 25.5 & 24.1 & 23.2 & 23.2 & 23.7 & 24.8 \\
\hline Other & 9.9 & 11.0 & 9.8 & 9.7 & 9.9 & 10.2 \\
\hline Capital & 5.7 & 5.0 & 4.8 & 4.3 & 3.2 & 2.7 \\
\hline Primary expenditure & 88.0 & 87.9 & 79.1 & 76.4 & 76.8 & 77.6 \\
\hline Wages & 24.1 & 23.9 & 22.0 & 21.1 & 20.8 & 20.8 \\
\hline Social benefits & 43.0 & 45.1 & 39.2 & 38.7 & 39.3 & 40.1 \\
\hline Goods and services & 11.2 & 8.7 & 8.1 & 7.1 & 7.1 & 7.2 \\
\hline Subsidies & 0.1 & 0.1 & 0.1 & 0.1 & 0.1 & 0.1 \\
\hline Other current & 2.1 & 2.9 & 2.3 & 2.3 & 2.4 & 2.3 \\
\hline Capital & 7.4 & 7.3 & 7.3 & 7.1 & 7.0 & 7.1 \\
\hline Unidentified measures (cumulative) & 0.0 & 0.0 & 0.0 & 0.0 & 2.8 & 3.9 \\
\hline Primary balance & -2.0 & -2.9 & 0.0 & 2.8 & 5.7 & 9.0 \\
\hline Interest payments $1 /$ & 12.8 & 10.1 & 8.4 & 9.0 & 9.9 & 10.2 \\
\hline Overall balance & -14.8 & -13.0 & -8.4 & -6.2 & -4.1 & -1.2 \\
\hline \multirow[t]{2}{*}{ Gross debt (Maastricht) } & 332.4 & 307.2 & 329.3 & 322.8 & 324.6 & 325.8 \\
\hline & \multicolumn{6}{|c|}{ (Percent of GDP) } \\
\hline Revenue & 42.2 & 43.6 & 42.9 & 42.8 & 41.8 & 41.3 \\
\hline Taxes on production and imports & 12.5 & 12.9 & 12.9 & 12.8 & 12.7 & 12.9 \\
\hline Taxes on income, and property & 9.5 & 10.1 & 9.5 & 9.9 & 9.8 & 9.6 \\
\hline Social contributions & 12.5 & 12.4 & 12.6 & 12.6 & 12.4 & 12.4 \\
\hline Other & 4.9 & 5.7 & 5.3 & 5.2 & 5.2 & 5.1 \\
\hline Capital transfers & 2.8 & 2.6 & 2.6 & 2.3 & 1.7 & 1.3 \\
\hline Primary expenditure & 43.2 & 45.1 & 42.9 & 41.3 & 40.2 & 38.7 \\
\hline Wages & 11.9 & 12.2 & 11.9 & 11.4 & 10.9 & 10.4 \\
\hline Social benefits & 21.1 & 23.1 & 21.3 & 20.9 & 20.6 & 20.0 \\
\hline Goods and services & 5.5 & 4.5 & 4.4 & 3.9 & 3.7 & 3.6 \\
\hline Subsidies & 0.1 & 0.1 & 0.1 & 0.1 & 0.1 & 0.1 \\
\hline Other current & 1.0 & 1.5 & 1.3 & 1.2 & 1.3 & 1.1 \\
\hline Capital & 3.6 & 3.7 & 4.0 & 3.8 & 3.7 & 3.5 \\
\hline Unidentified measures (cumulative) & 0.0 & 0.0 & 0.0 & 0.0 & 1.5 & 2.0 \\
\hline Primary balance & -1.0 & -1.5 & 0.0 & 1.5 & 3.0 & 4.5 \\
\hline Interest payments $1 /$ & 6.3 & 5.2 & 4.5 & 4.9 & 5.2 & 5.1 \\
\hline Overall balance & -7.3 & -6.7 & -4.5 & -3.4 & -2.2 & -0.6 \\
\hline Gross debt (Maastricht) & 163.2 & 157.5 & 178.5 & 174.5 & 170.0 & 162.7 \\
\hline Nominal GDP (billions of euros) & 203.7 & 195.0 & 184.5 & 184.9 & 191.0 & 200.2 \\
\hline
\end{tabular}

Sources: Ministry of Finance; and IMF staff projections.

$1 /$ Reflects all debt relief measures that have accrual effects. 


\begin{tabular}{|c|c|c|c|c|}
\hline \multicolumn{5}{|c|}{$\begin{array}{c}\text { Table 15. Greece: Authorities" Measures to Meet the 2013-14 Targets } \\
\text { (Percent of GDP) }\end{array}$} \\
\hline \multicolumn{2}{|c|}{ Total net impact } & 2013 & 2014 & 2013-14 \\
\hline \multicolumn{2}{|c|}{ Expenditure measures } & 4.0 & 1.2 & 5.2 \\
\hline \multirow[t]{3}{*}{ I } & $\begin{array}{l}\text { Public administration restructuring } \\
\text { Of which: }\end{array}$ & 0.2 & 0.2 & 0.4 \\
\hline & Permanent cuts in PIB (excl. sub-national governments) & 0.1 & 0.1 & 0.2 \\
\hline & $25 \%$ reduction in discretionary non-wage spending & 0.1 & 0.0 & 0.2 \\
\hline \multirow[t]{5}{*}{ II } & $\begin{array}{l}\text { Public sector wage bill } \\
\text { Of which: }\end{array}$ & 0.6 & 0.1 & 0.7 \\
\hline & Rationalization of special wage regimes & 0.1 & 0.0 & 0.1 \\
\hline & Cuts in public sector seasonal bonus & 0.2 & 0.0 & 0.2 \\
\hline & Introduction of mobility scheme & 0.0 & 0.0 & 0.1 \\
\hline & Suspension of the performance and fiscal bonus in the public sector & 0.2 & 0.0 & 0.2 \\
\hline \multirow[t]{9}{*}{ III } & $\begin{array}{l}\text { Pensions } \\
\text { Of which: }\end{array}$ & 2.6 & 0.2 & 2.8 \\
\hline & Increase in retirement age by two years & 0.3 & 0.1 & 0.5 \\
\hline & Rationalization of lump-sum pensions of the public sector 2013 and 2014 & 0.1 & 0.0 & 0.1 \\
\hline & $\begin{array}{l}\text { Reduction in supplementary and main pensions (between } 1000-1500 \text { by } 5 \% \text {, } \\
1500-2000 \text { by } 10 \%, 2000-3000 \text { by } 15 \%, 3000-4000 \text { by } 20 \%,>4000 \text { by } 25 \%\end{array}$ & 0.6 & 0.0 & 0.6 \\
\hline & Elimination of seasonal bonuses of supplementary and main pensions, excl. OGA & 0.9 & 0.0 & 0.9 \\
\hline & Elimination of seasonal bonuses of main pensions, OGA & 0.4 & 0.0 & 0.4 \\
\hline & Application of census on pensioners & 0.0 & 0.0 & 0.0 \\
\hline & Cuts in pensions due to rationalization of special wage regimes & 0.1 & 0.0 & 0.1 \\
\hline & Cuts in pensions of armed forces due to new wage bill & 0.0 & 0.1 & 0.1 \\
\hline IV & Social benefits & 0.0 & 0.0 & 0.0 \\
\hline \multirow[t]{4}{*}{ v } & Health & 0.2 & 0.3 & 0.6 \\
\hline & Of which: & 0.0 & 0.0 & 0.0 \\
\hline & Reduction in pharmaceutical expenditure of social security funds & 0.2 & 0.2 & 0.4 \\
\hline & Increase in co-payments & 0.0 & 0.1 & 0.1 \\
\hline VI & Defense & 0.2 & 0.1 & 0.2 \\
\hline & Education & 0.0 & 0.0 & 0.1 \\
\hline VIII & SoEs rationalization & 0.1 & 0.1 & 0.2 \\
\hline & Local governments & 0.0 & 0.1 & 0.1 \\
\hline \multicolumn{2}{|c|}{ Revenue Measures } & 1.0 & 0.9 & 1.9 \\
\hline & Of which: & & & \\
\hline \multirow[t]{5}{*}{ I } & Income tax reform & 0.2 & 0.8 & 1.0 \\
\hline & Restructuring of PIT and CIT rates and new tax regime for self-employed & 0.1 & 0.4 & 0.6 \\
\hline & Reductions in individuals' income tax credits & 0.0 & 0.1 & 0.1 \\
\hline & Reductions in family allowances & 0.1 & 0.1 & 0.2 \\
\hline & Increase in tax on deposit interest from $10 \%$ to $15 \%$ & 0.0 & 0.1 & 0.1 \\
\hline II & Reduction of VAT refunds to farmers & 0.1 & 0.0 & 0.1 \\
\hline III & Excises & 0.2 & 0.0 & 0.2 \\
\hline IV & Taxation by tonnage of Greek owned merchant fleet & 0.0 & 0.0 & 0.1 \\
\hline V & Equalization of social security contributions ceiling & 0.3 & 0.0 & 0.3 \\
\hline VI & Taxes on lottery games and winners gains & 0.2 & 0.0 & 0.2 \\
\hline Total & & 5.0 & 2.1 & 7.2 \\
\hline
\end{tabular}


Table 16. Greece: Income Tax Reform

\begin{tabular}{|c|c|c|}
\hline & Pre-Reform & Post-Reform \\
\hline \multicolumn{3}{|l|}{ Personal income tax } \\
\hline Number of tax rates & 8 & 3 \\
\hline Tax credit & & $€ 2,100$ \\
\hline Standard deduction & $€ 5,000$ & $\ldots$ \\
\hline \multicolumn{3}{|l|}{ Other deductions } \\
\hline Mortgage interest & ○ & \\
\hline Rental payment & ( & \\
\hline Tuition fees & $\odot$ & \\
\hline Insurance premia & ( & \\
\hline Renewable energy & ( & \\
\hline Premium amount forfeit & $\odot$ & \\
\hline Child care expenses & 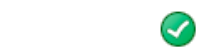 & \\
\hline Medical costs & ( & ( \\
\hline Donations & $\odot$ & ○ \\
\hline Sponsorships & 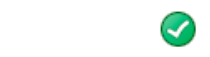 & 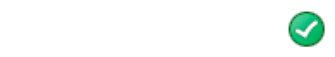 \\
\hline Alimony & 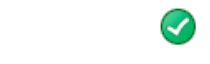 & 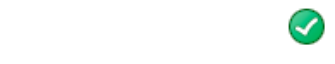 \\
\hline Child tax allowance & ○ & \\
\hline Farmers & & $13 \%$ \\
\hline Sailors, crew & $3 \%$ & $10 \%$ \\
\hline Sailors, officers & $6 \%$ & $15 \%$ \\
\hline \multicolumn{3}{|l|}{ Corporate income tax } \\
\hline Self-employed & Under PIT & \\
\hline Number of tax rates & 8 & 2 \\
\hline Standard deduction & $€ 5,000$ & $\ldots$ \\
\hline \multicolumn{3}{|l|}{ Incorporated enterprises } \\
\hline Corporate taxable income & $20 \%$ & $26 \%$ \\
\hline Distributed profits & $25 \%$ & $10 \%$ \\
\hline \multicolumn{3}{|l|}{ Capital income tax } \\
\hline \multirow[t]{2}{*}{ Rental income } & Under PIT & $10 \%$ up to $€ 12,000$ \\
\hline & & $33 \%$ above $€ 12,000$ \\
\hline Interest income on deposits & $10 \%$ & $15 \%$ \\
\hline
\end{tabular}




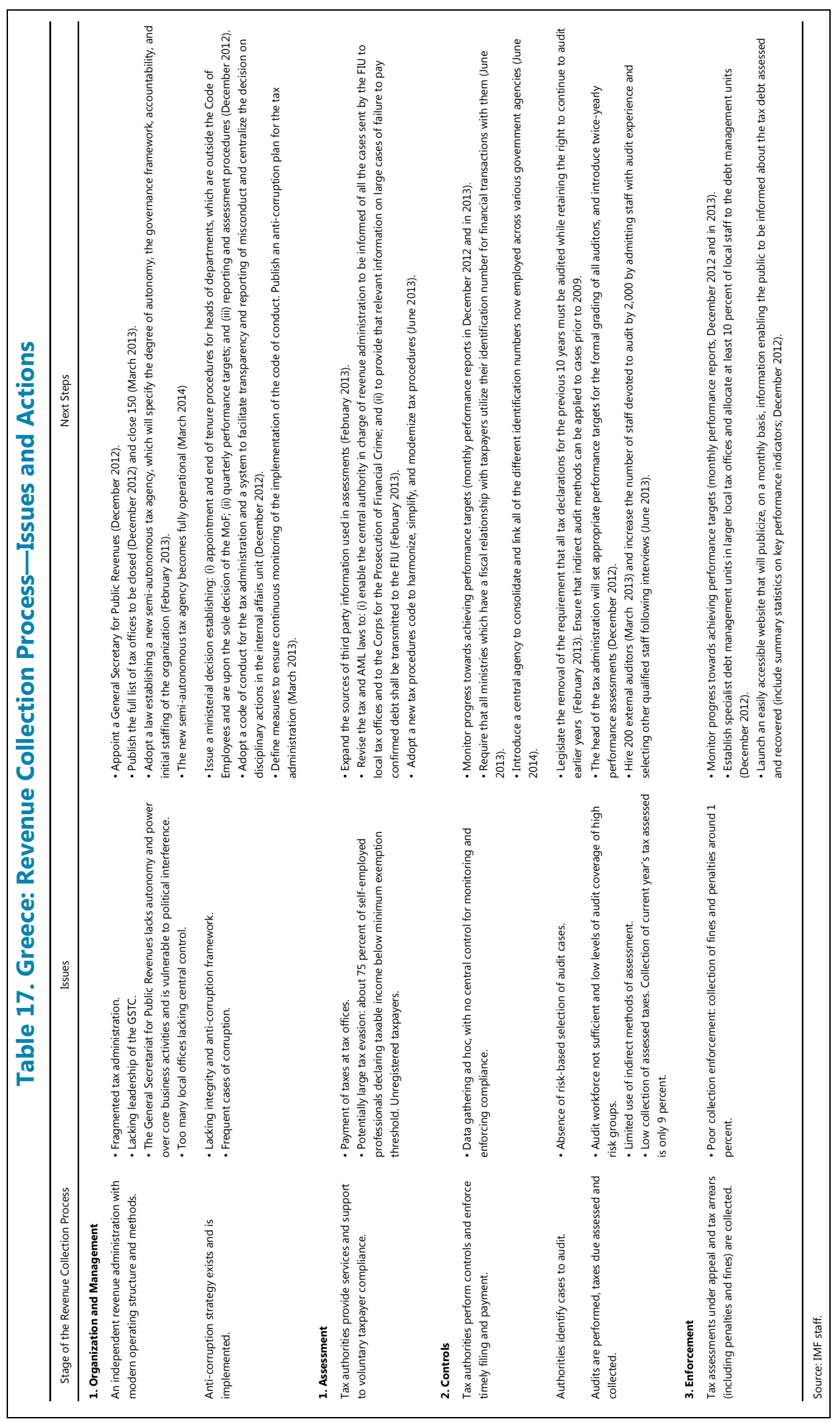




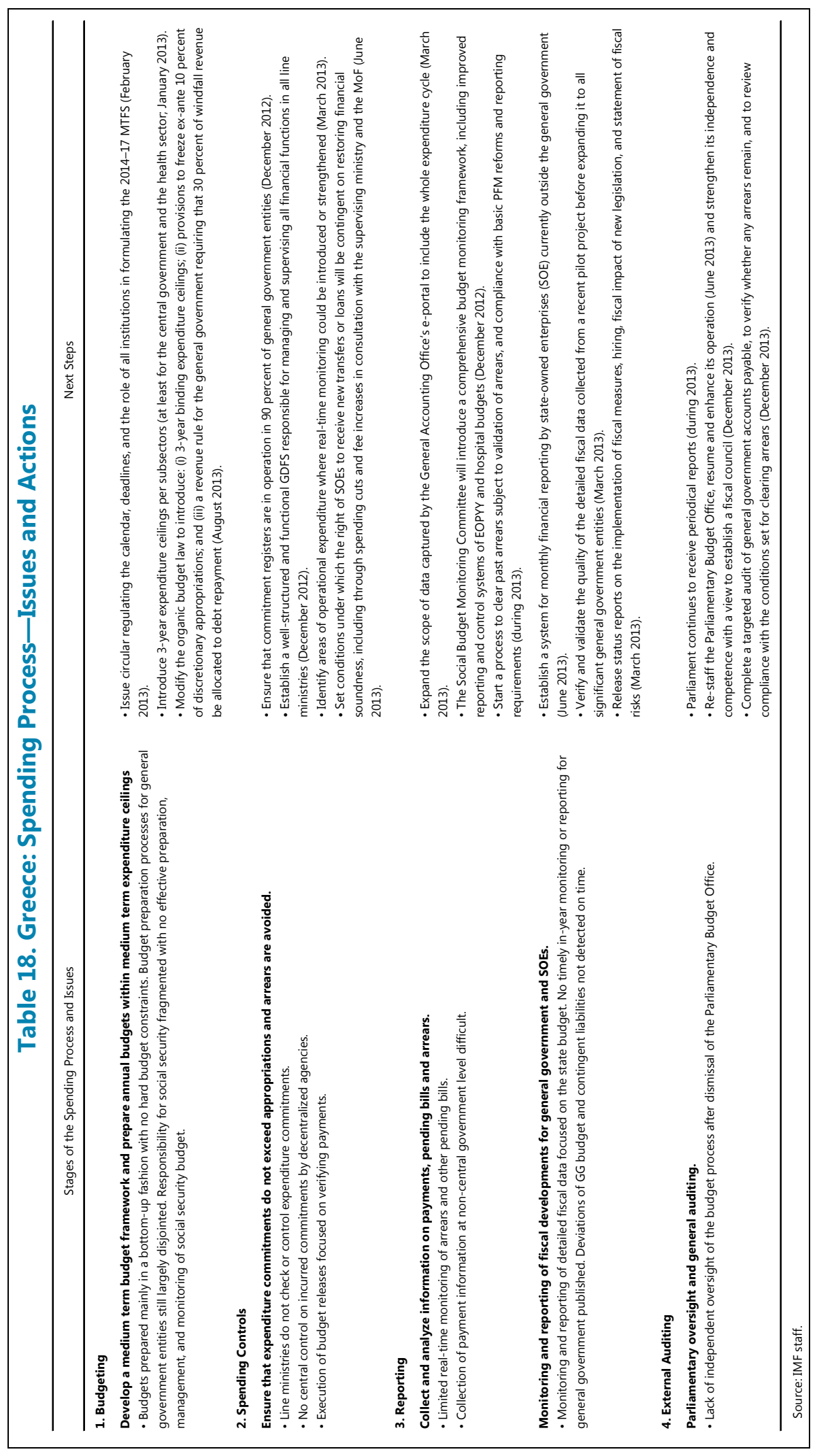

INTERNATIONAL MONETARY FUND 


\begin{tabular}{|c|c|c|c|c|c|c|c|}
\hline \multicolumn{8}{|c|}{$\begin{array}{r}\text { Table 19. Greece: General Government Financing R } \\
\text { (Billion of euros, unless otherwise indicated) }\end{array}$} \\
\hline & 2010 & 2011 & 2012 & 2013 & 2014 & 2015 & 2016 \\
\hline & & & \multicolumn{5}{|c|}{ Projections } \\
\hline Gross borrowing need & 60.8 & 59.2 & 127.1 & 56.7 & 33.3 & 33.3 & 23.9 \\
\hline Overall balance (accrual) & 23.9 & 20.0 & 13.0 & 8.4 & 6.2 & 4.1 & 1.2 \\
\hline Amortization & 28.5 & 37.3 & 24.4 & 27.4 & 35.4 & 25.8 & 17.4 \\
\hline Medium and long-term (non-official) & 19.6 & 28.1 & 12.7 & 9.0 & 15.7 & 6.0 & 3.1 \\
\hline Residents & 3.9 & 5.6 & 2.1 & 0.0 & 6.7 & -0.1 & 0.4 \\
\hline Non-residents & 15.6 & 22.5 & 10.6 & 9.0 & 9.1 & 6.1 & 2.7 \\
\hline Short-term & 8.9 & 9.2 & 11.8 & 16.8 & 12.3 & 11.3 & 11.3 \\
\hline Residents & 0.7 & 8.0 & 10.0 & 15.0 & 10.5 & 9.5 & 9.5 \\
\hline Non-residents & 8.2 & 1.2 & 1.8 & 1.8 & 1.8 & 1.8 & 1.8 \\
\hline Official creditors & 0.0 & 0.0 & 0.0 & 1.7 & 7.4 & 8.6 & 3.1 \\
\hline IMF & 0.0 & 0.0 & 0.0 & 1.7 & 7.4 & 8.6 & 3.1 \\
\hline $\mathrm{EC}$ & 0.0 & 0.0 & 0.0 & 0.0 & 0.0 & 0.0 & 0.0 \\
\hline Other & 8.4 & 1.9 & 89.6 & 20.9 & -8.3 & 3.3 & 5.3 \\
\hline Of which: bank recapitalization & $\ldots$ & $\ldots$ & 44.2 & 4.0 & 0.0 & 0.0 & 0.0 \\
\hline Of which: PSI-related costs & $\ldots$ & $\ldots$ & 34.5 & 0.0 & 0.0 & 0.0 & 0.0 \\
\hline Gross financing sources & 60.8 & 59.2 & 127.1 & 17.5 & 15.6 & 15.3 & 15.0 \\
\hline Privatization receipts & 0.0 & 1.0 & 0.1 & 2.5 & 1.9 & 2.0 & 2.0 \\
\hline Market access & 28.5 & 11.8 & 16.8 & 12.3 & 11.3 & 11.3 & 11.3 \\
\hline Medium and long-term & 19.3 & 0.0 & 0.0 & 0.0 & 0.0 & 0.0 & 0.0 \\
\hline Residents & 19.3 & 0.0 & 0.0 & 0.0 & 0.0 & 0.0 & 0.0 \\
\hline Non-residents & 0.0 & 0.0 & 0.0 & 0.0 & 0.0 & 0.0 & 0.0 \\
\hline Short-term & 9.2 & 11.8 & 16.8 & 12.3 & 11.3 & 11.3 & 11.3 \\
\hline Residents & 8.0 & 11.8 & 15.0 & 10.5 & 9.5 & 9.5 & 9.5 \\
\hline Non-residents & 1.2 & 0.0 & 1.8 & 1.8 & 1.8 & 1.8 & 1.8 \\
\hline Other & 0.9 & 4.5 & 0.3 & 2.7 & 2.5 & 2.0 & 1.7 \\
\hline Official financing already disbursed & 31.4 & 41.9 & 109.9 & 0.0 & 0.0 & 0.0 & 0.0 \\
\hline EC bilateral loans/EAMS & 21.1 & 32.0 & 108.3 & 0.0 & 0.0 & 0.0 & 0.0 \\
\hline IMF & 10.3 & 9.9 & 1.6 & 0.0 & 0.0 & 0.0 & 0.0 \\
\hline Financing gap (including not yet disbursed) & 0.0 & 0.0 & 0.0 & 39.3 & 17.7 & 18.0 & 8.9 \\
\hline Official financing $1 /$ & $\ldots$ & 0.0 & 0.0 & 39.2 & 17.6 & 10.9 & 6.4 \\
\hline EAMS & $\ldots$ & 0.0 & 0.0 & 28.8 & 10.5 & 3.8 & 4.6 \\
\hline Of which: interest deferral & & & & 1.2 & 1.9 & 3.8 & 4.6 \\
\hline IMF & $\ldots$ & 0.0 & 0.0 & 10.4 & 7.1 & 7.1 & 1.8 \\
\hline Other 2/ & $\ldots$ & 0.0 & 0.0 & 0.0 & 0.1 & 7.1 & 2.5 \\
\hline \multicolumn{8}{|l|}{ Memorandum items: } \\
\hline $\begin{array}{l}\text { Total Maastricht debt } \\
\text { Of which: }\end{array}$ & 329.0 & 355.8 & 307.2 & 329.3 & 322.8 & 324.6 & 325.8 \\
\hline Official creditors & 31.0 & 73.8 & 183.6 & 221.1 & 231.4 & 233.7 & 237.0 \\
\hline IMF & 10.0 & 20.7 & 22.3 & 31.0 & 30.7 & 29.2 & 27.9 \\
\hline (percent of quota) & 845 & 1592 & 1719 & 2386 & 2364 & 2248 & 2148 \\
\hline EAMS & 21.0 & 53.1 & 161.3 & 190.2 & 200.7 & 204.5 & 209.1 \\
\hline Private sector & 297.0 & 282.0 & 123.5 & 108.1 & 91.4 & 83.8 & 79.2 \\
\hline Unidentified official financing/market access & $\ldots$ & $\ldots$ & 0.0 & 0.0 & 0.0 & 7.1 & 9.6 \\
\hline Total Maastricht debt (percent of GDP) & 145.0 & 170.6 & 157.5 & 178.5 & 174.5 & 170.0 & 162.7 \\
\hline \multicolumn{8}{|l|}{ Of which: } \\
\hline Official creditors & 14.0 & 35.4 & 94.2 & 119.8 & 125.1 & 122.4 & 118.4 \\
\hline IMF & 5.0 & 9.9 & 11.4 & 16.8 & 16.6 & 15.3 & 13.9 \\
\hline EAMS & 9.0 & 25.5 & 82.7 & 103.1 & 108.5 & 107.1 & 104.4 \\
\hline Private sector & 131.0 & 135.2 & 63.4 & 58.6 & 49.4 & 43.9 & 39.5 \\
\hline Unidentified official financing/market access & $\ldots$ & $\ldots$ & 0.0 & 0.0 & 0.0 & 3.7 & 4.8 \\
\hline \multicolumn{8}{|l|}{ Sources: Ministry of Finance; and IMF staff projections. } \\
\hline $\begin{array}{l}\text { 1/ Official financing includes committed funds. Int } \\
2 \text { / Includes unidentified financing. }\end{array}$ & top & EUR & in $c$ & tted fund & the $\mathrm{E}$ & & \\
\hline
\end{tabular}




\begin{tabular}{|c|c|c|c|c|c|c|c|c|}
\hline \multicolumn{9}{|c|}{$\begin{array}{r}\text { Table 20. Greece: External Financing Requirements } \\
\text { (Billions of euros, unless otherwise indicated) }\end{array}$} \\
\hline & 2010 & 2011 & 2012 & 2013 & 2014 & 2015 & 2016 & 2017 \\
\hline & & & \multicolumn{6}{|c|}{ Proj. } \\
\hline Gross financing requirements & 216.2 & 239.0 & 202.8 & 185.8 & 187.5 & 181.9 & 159.5 & 148.7 \\
\hline Current account deficit & 22.5 & 20.6 & 8.1 & 2.3 & 0.5 & -0.8 & -1.4 & -1.8 \\
\hline Medium and long-term debt amortization & 22.7 & 35.5 & 13.1 & 13.3 & 19.0 & 17.2 & 8.3 & 8.7 \\
\hline Public sector & 15.6 & 22.5 & 10.6 & 10.7 & 16.5 & 14.7 & 5.8 & 6.2 \\
\hline Of which: EC/IMF & 0.0 & 0.0 & 0.0 & 1.7 & 7.4 & 8.6 & 3.1 & 0.8 \\
\hline Banks & 6.7 & 10.7 & 1.2 & 1.2 & 1.2 & 1.2 & 1.2 & 1.2 \\
\hline Other & 0.3 & 2.3 & 1.4 & 1.3 & 1.3 & 1.3 & 1.3 & 1.3 \\
\hline Short-term debt amortization & 171.1 & 182.9 & 181.5 & 170.3 & 168.1 & 165.4 & 152.6 & 141.8 \\
\hline Public sector and Bank of Greece & 57.2 & 88.2 & 106.5 & 106.5 & 105.3 & 102.7 & 88.8 & 76.7 \\
\hline Bank of Greece 1/ & 49.0 & 87.1 & 104.8 & 104.8 & 103.6 & 100.9 & 87.1 & 75.0 \\
\hline Public sector & 8.2 & 1.2 & 1.8 & 1.8 & 1.8 & 1.8 & 1.8 & 1.8 \\
\hline Banks 2/ & 113.2 & 93.4 & 74.4 & 63.3 & 62.3 & 62.4 & 63.3 & 64.7 \\
\hline Other & 0.6 & 1.3 & 0.6 & 0.5 & 0.4 & 0.4 & 0.4 & 0.4 \\
\hline Source of financing & 184.7 & 197.4 & 92.9 & 146.6 & 169.9 & 163.9 & 150.6 & 143.3 \\
\hline Capital account (net) & 2.1 & 2.7 & 3.9 & 3.9 & 3.5 & 3.3 & 3.3 & 3.3 \\
\hline Foreign direct investment (net) & -0.9 & -0.5 & 1.1 & 1.7 & 1.8 & 1.9 & 1.8 & 2.1 \\
\hline Equities (net) & -2.3 & -0.2 & 0.0 & 0.0 & 0.1 & 0.6 & 1.1 & 1.3 \\
\hline Assets drawdown (- increase) & 21.7 & 12.4 & -83.6 & -28.2 & -2.2 & 4.2 & 1.1 & 0.0 \\
\hline New borrowing and debt rollover & 164.1 & 183.5 & 171.6 & 169.3 & 166.7 & 153.8 & 143.2 & 136.6 \\
\hline Medium and long-term borrowing & -18.8 & 2.0 & 1.2 & 1.2 & 1.2 & 1.2 & 1.4 & 1.5 \\
\hline Public sector $3 /$ & -5.7 & -3.1 & 0.0 & 0.0 & 0.0 & 0.0 & 0.0 & 0.0 \\
\hline Banks & -12.3 & 3.8 & 0.0 & 0.0 & 0.0 & 0.0 & 0.1 & 0.3 \\
\hline Other & -0.8 & 1.2 & 1.2 & 1.2 & 1.2 & 1.2 & 1.2 & 1.3 \\
\hline Short-term borrowing & 182.9 & 181.5 & 170.3 & 168.1 & 165.4 & 152.6 & 141.8 & 135.1 \\
\hline Public sector and Bank of Greece & 88.2 & 106.5 & 106.5 & 105.3 & 102.7 & 88.8 & 76.7 & 69.7 \\
\hline Bank of Greece 1/ & 87.1 & 104.8 & 104.8 & 103.6 & 100.9 & 87.1 & 75.0 & 67.2 \\
\hline Public sector 4/ & 1.2 & 1.8 & 1.8 & 1.8 & 1.8 & 1.8 & 1.8 & 2.5 \\
\hline Banks 2/ & 93.4 & 74.4 & 63.3 & 62.3 & 62.4 & 63.3 & 64.7 & 64.9 \\
\hline Other & 1.3 & 0.6 & 0.5 & 0.4 & 0.4 & 0.4 & 0.4 & 0.5 \\
\hline Other & 0.1 & -0.6 & 0.0 & 0.0 & 0.0 & 0.0 & 0.0 & 0.0 \\
\hline Program financing & 31.5 & 41.5 & 109.9 & 39.2 & 17.6 & 10.9 & 6.4 & 5.4 \\
\hline Unidentified official financing / market access & 0.0 & 0.0 & 0.0 & 0.0 & 0.0 & 7.1 & 2.5 & 0.0 \\
\hline $\begin{array}{l}\text { Sources: Bank of Greece; Bloomberg; and IMF st } \\
\text { 1/ Includes liabilities to Eurosystem related to T } \\
\text { 2/ Includes currency and deposits and securitize } \\
\text { 3/ Actual figures on public sector medium and I } \\
\text { 4/ Includes government deposits' build-up (reg }\end{array}$ & $\begin{array}{l}\text { wing are b } \\
\text { ncy denomi }\end{array}$ & $\begin{array}{l}\text { ed on nor } \\
\text { ation for } p\end{array}$ & $\begin{array}{l}\text { residents } \\
\text { sentation }\end{array}$ & $\begin{array}{l}\text { nortizatic } \\
\text { purpose }\end{array}$ & figures $p r$ & ided by & OO and & \\
\hline
\end{tabular}




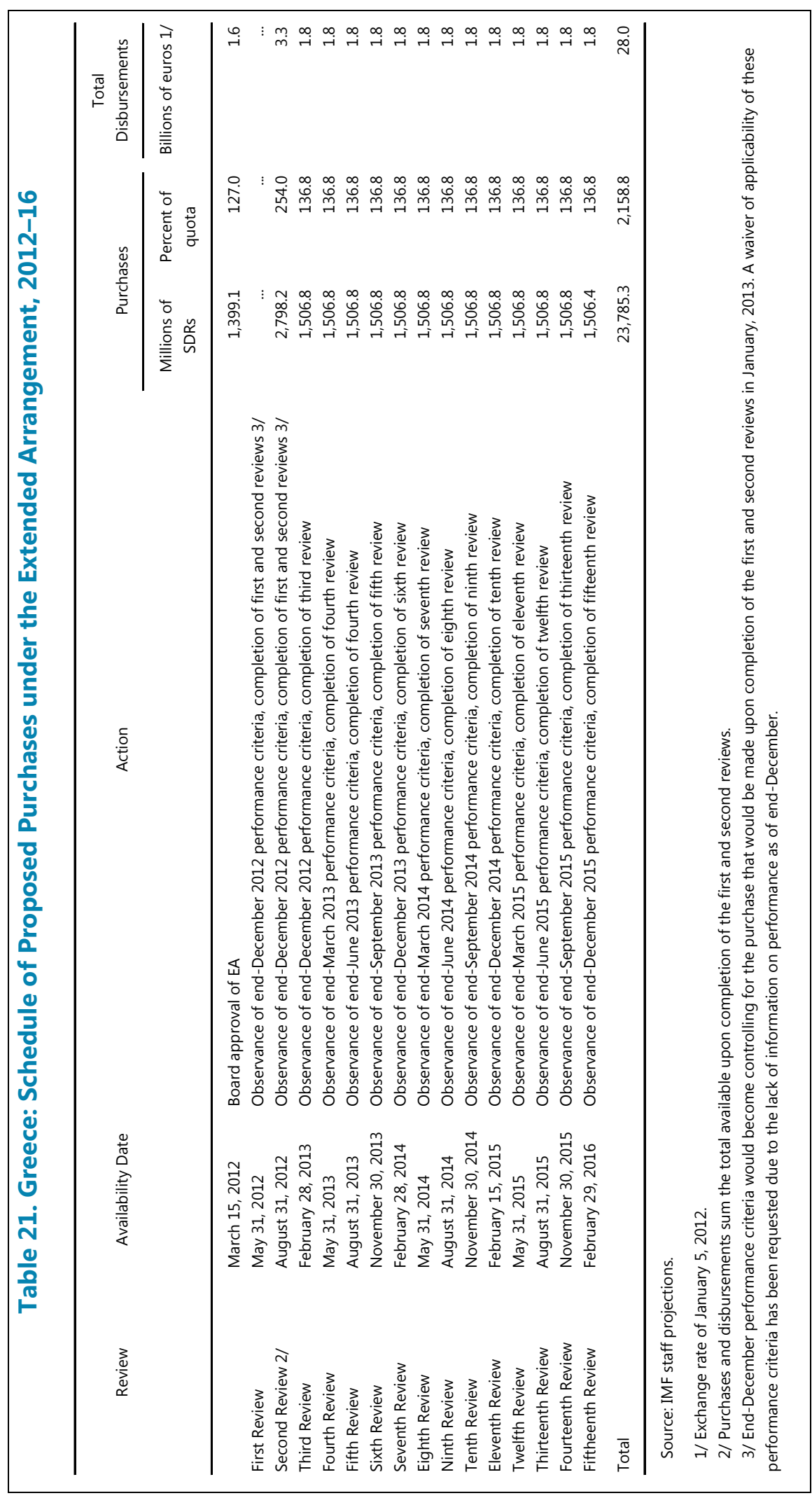




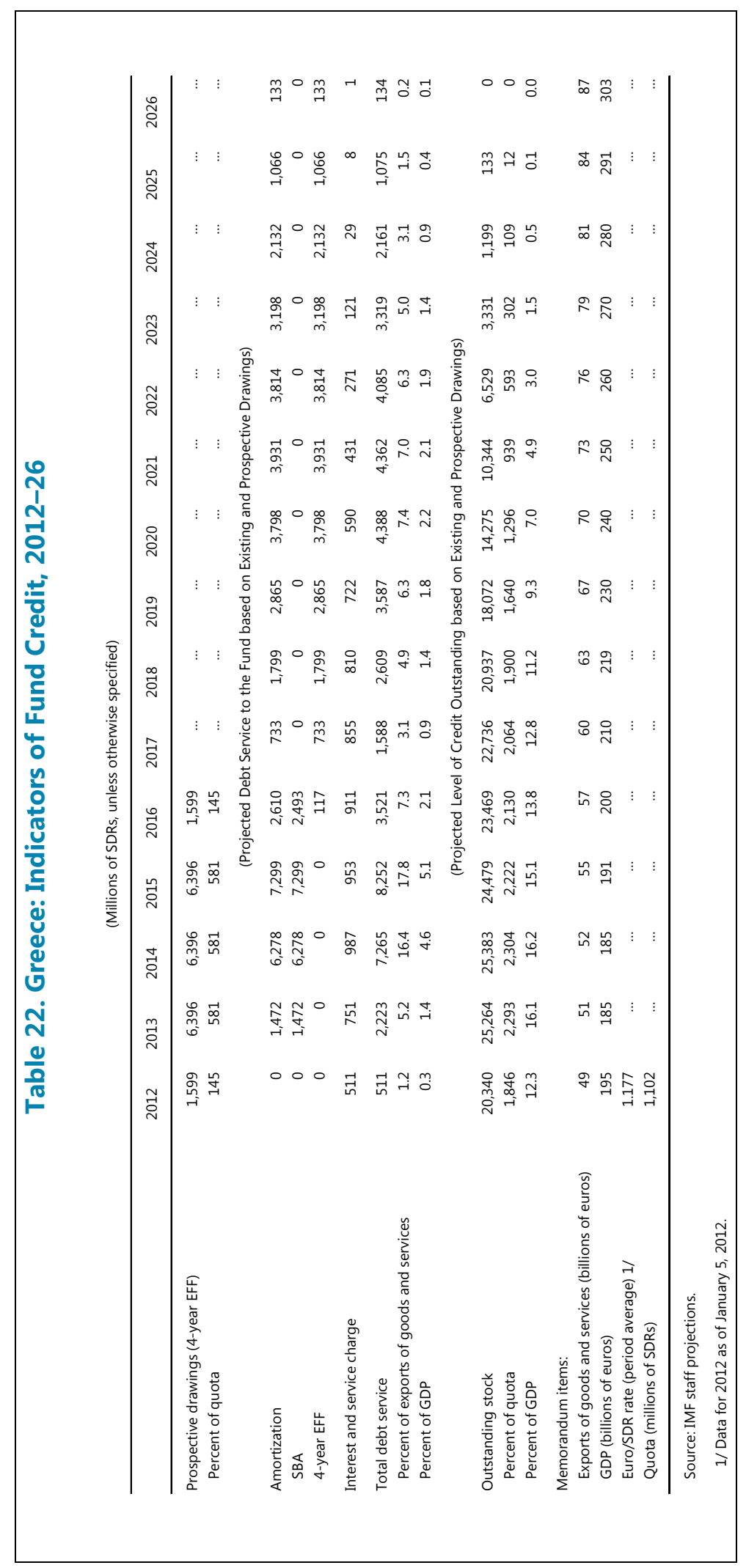


Figure 1. Greece: Selected Economic Indicators, 2005-12

(Year-on-year percent change, unless otherwise indicated)
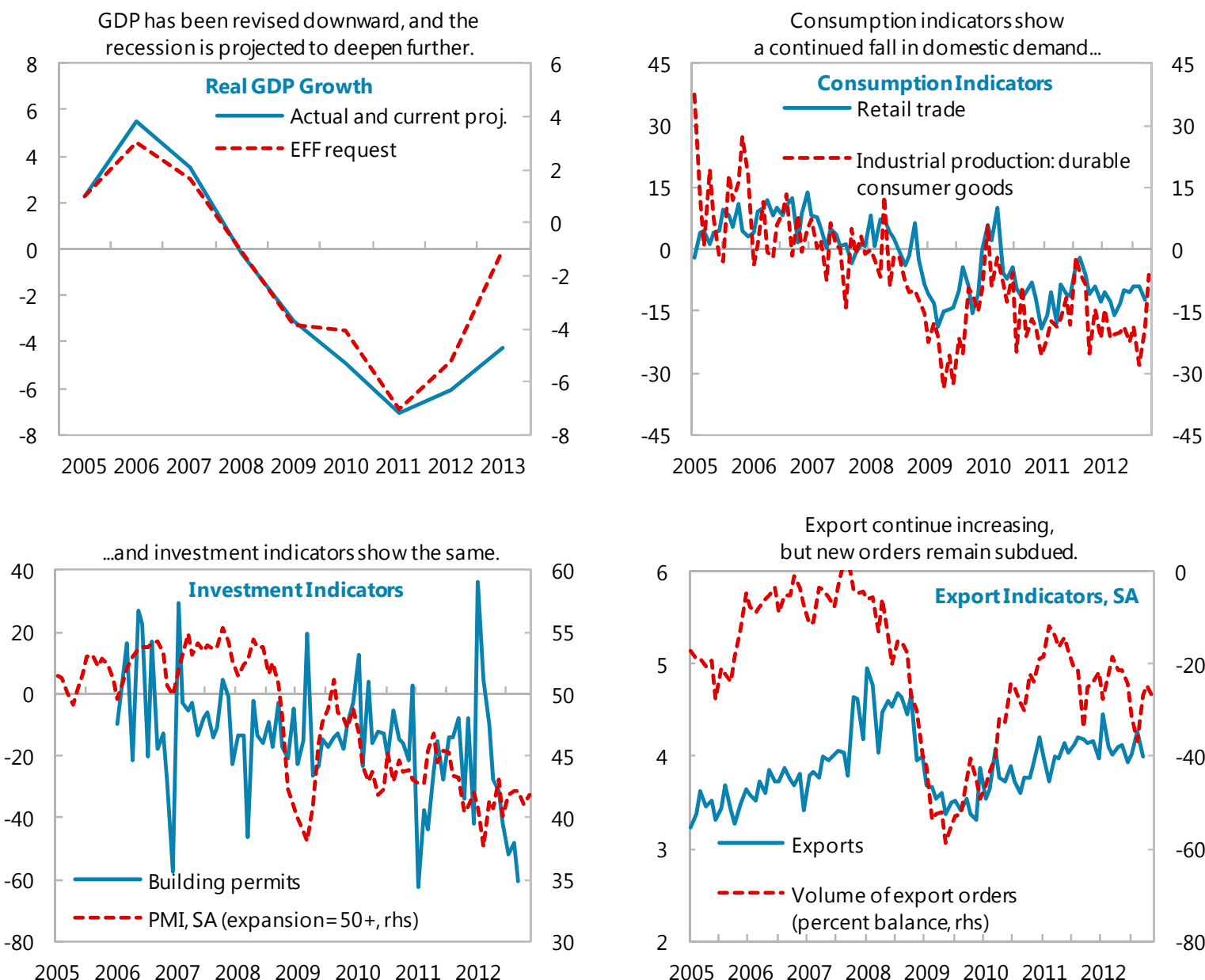

Export continue increasing,
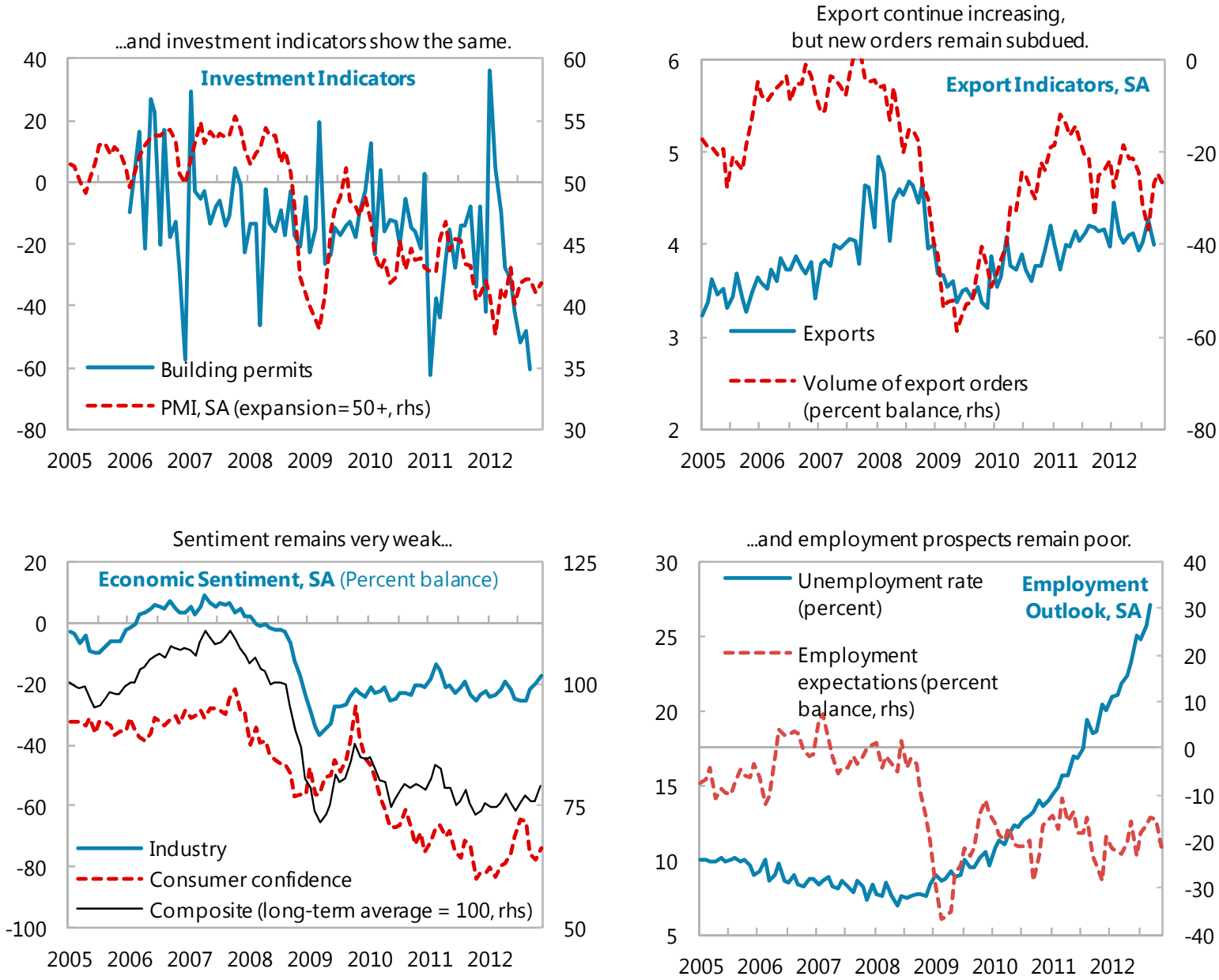

Sources: Elstat; Eurostat; European Commission; and IMF staff calculations. 
Figure 2. Greece: Inflation Developments, 2005-12

\section{(Year-on-year percent change)}
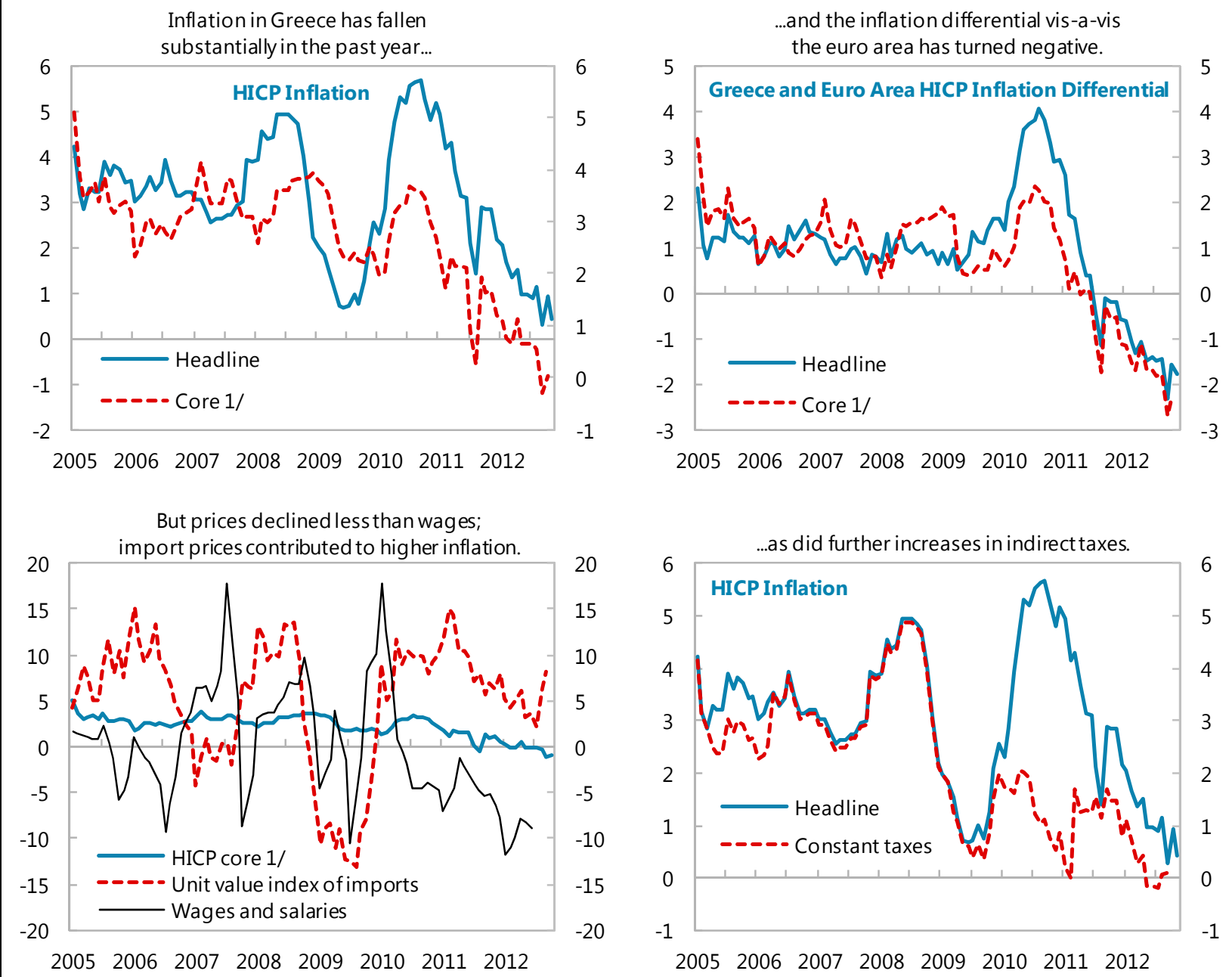

Sources: Elstat; Eurostat; Haver; and IMF staff calculations.

1/ Excludes food, alcohol, tobacco, and energy. 
Figure 3. Greece: Competitiveness Indicators, 2005-12
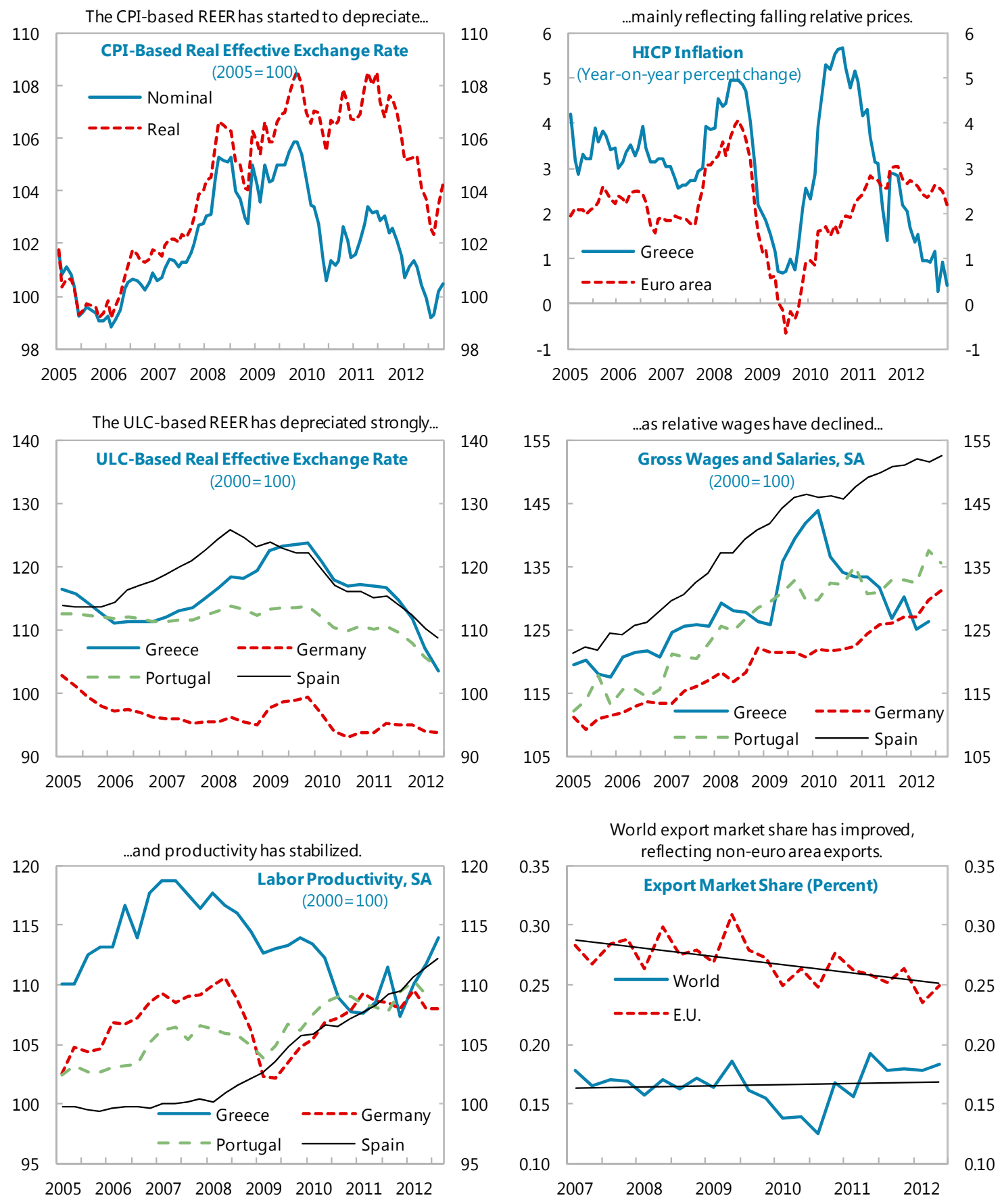

Sources: Haver; Eurostat; IMF, Direction of Trade Statistics; and IMF staff calculations. 


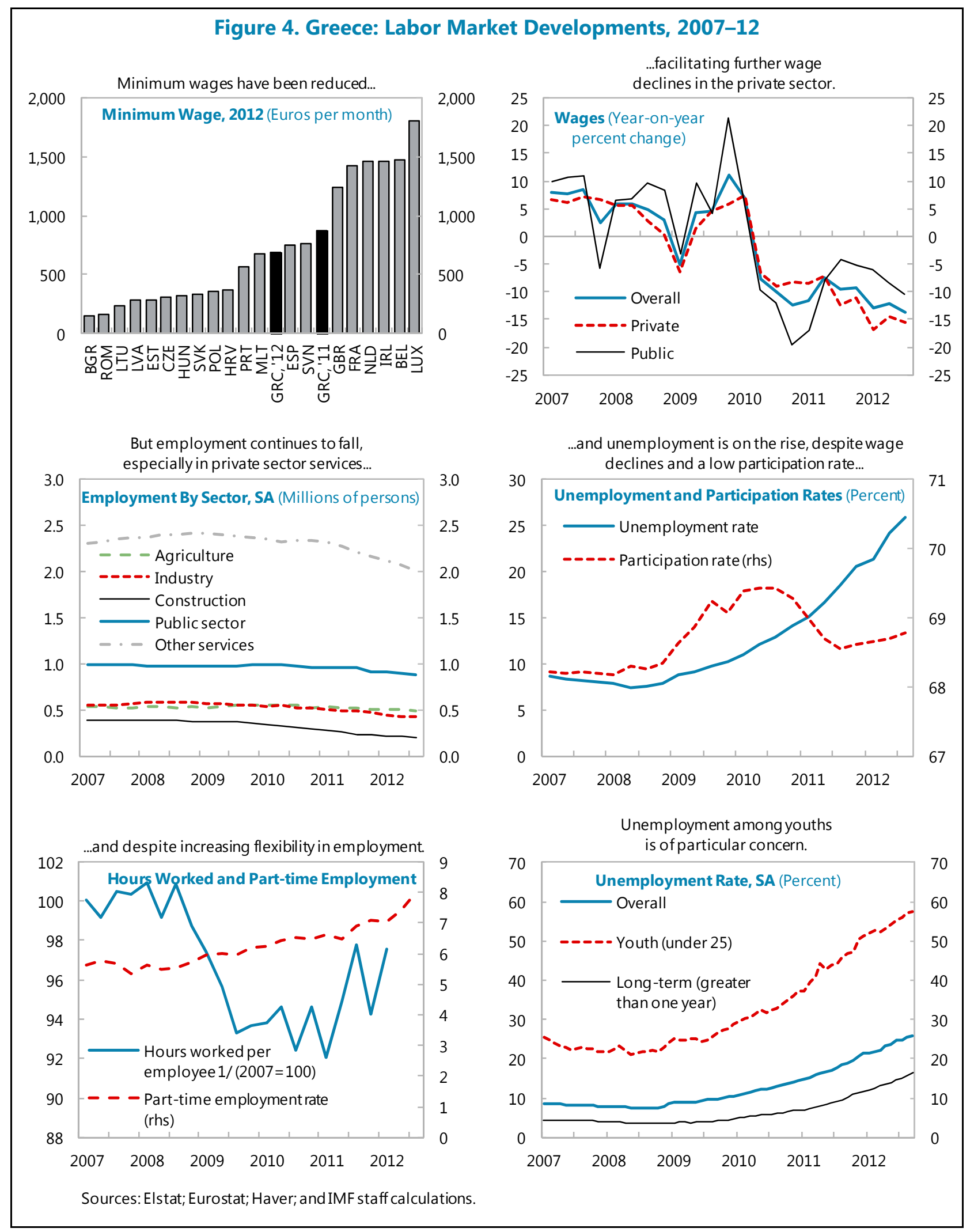




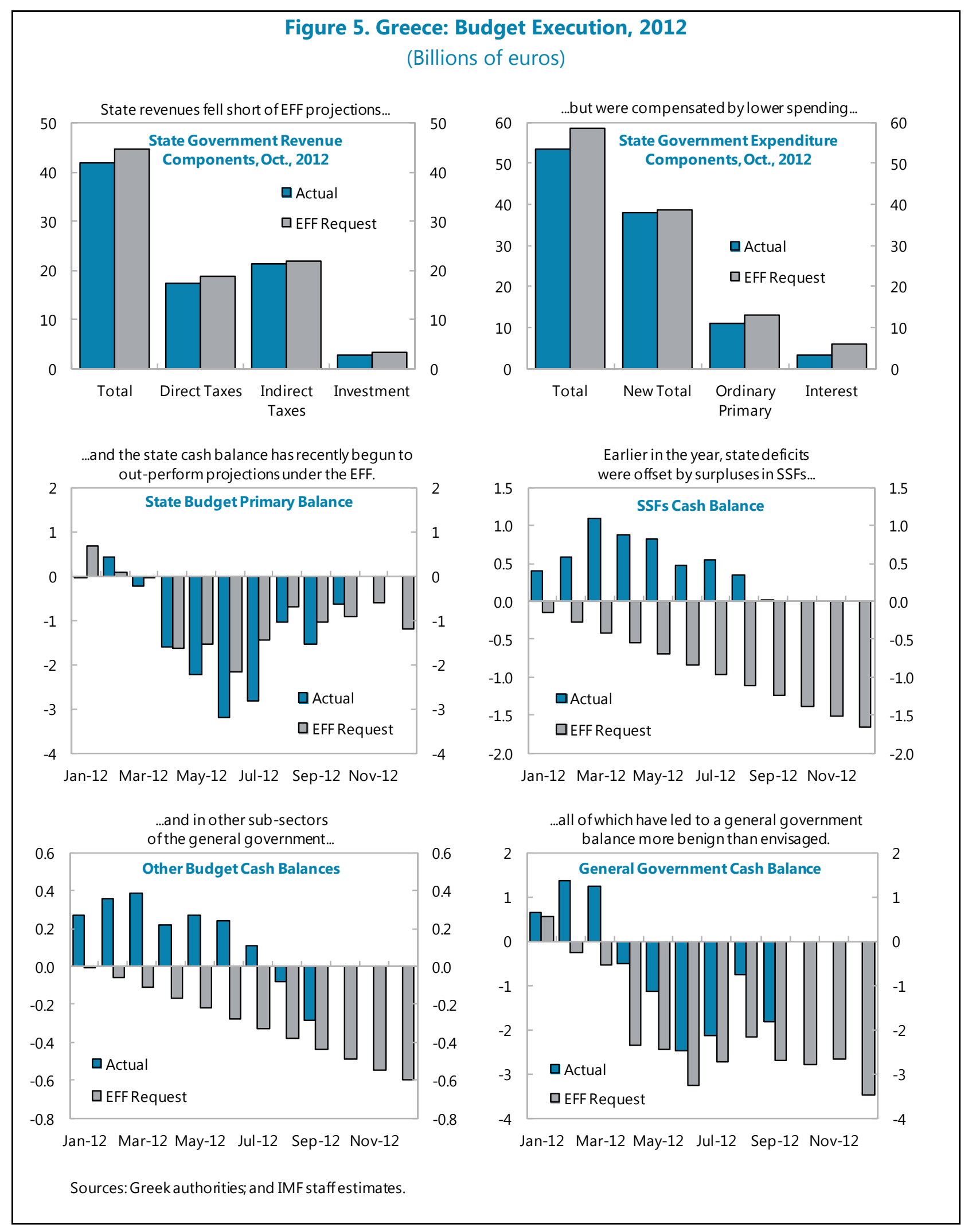


Figure 6. Greece: Balance of Payments Developments, 2010-12

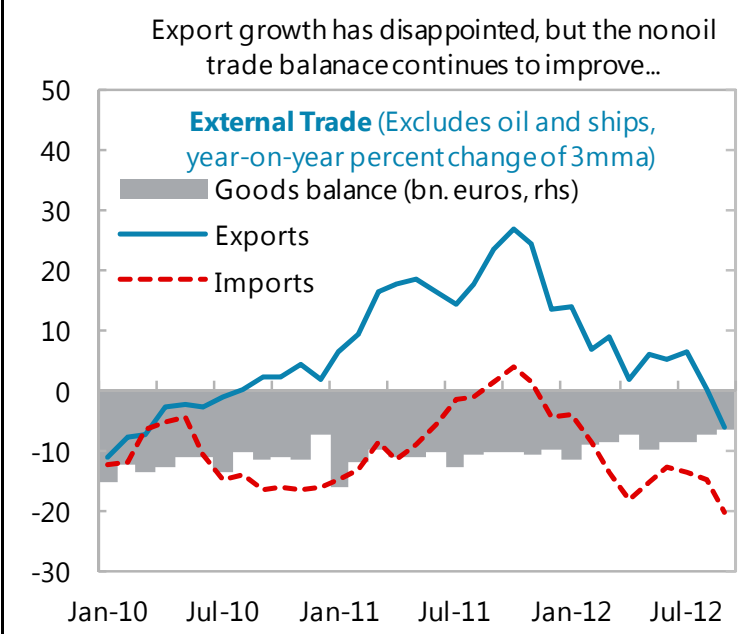

10
8
6
4
2
0
-2
-4
-6
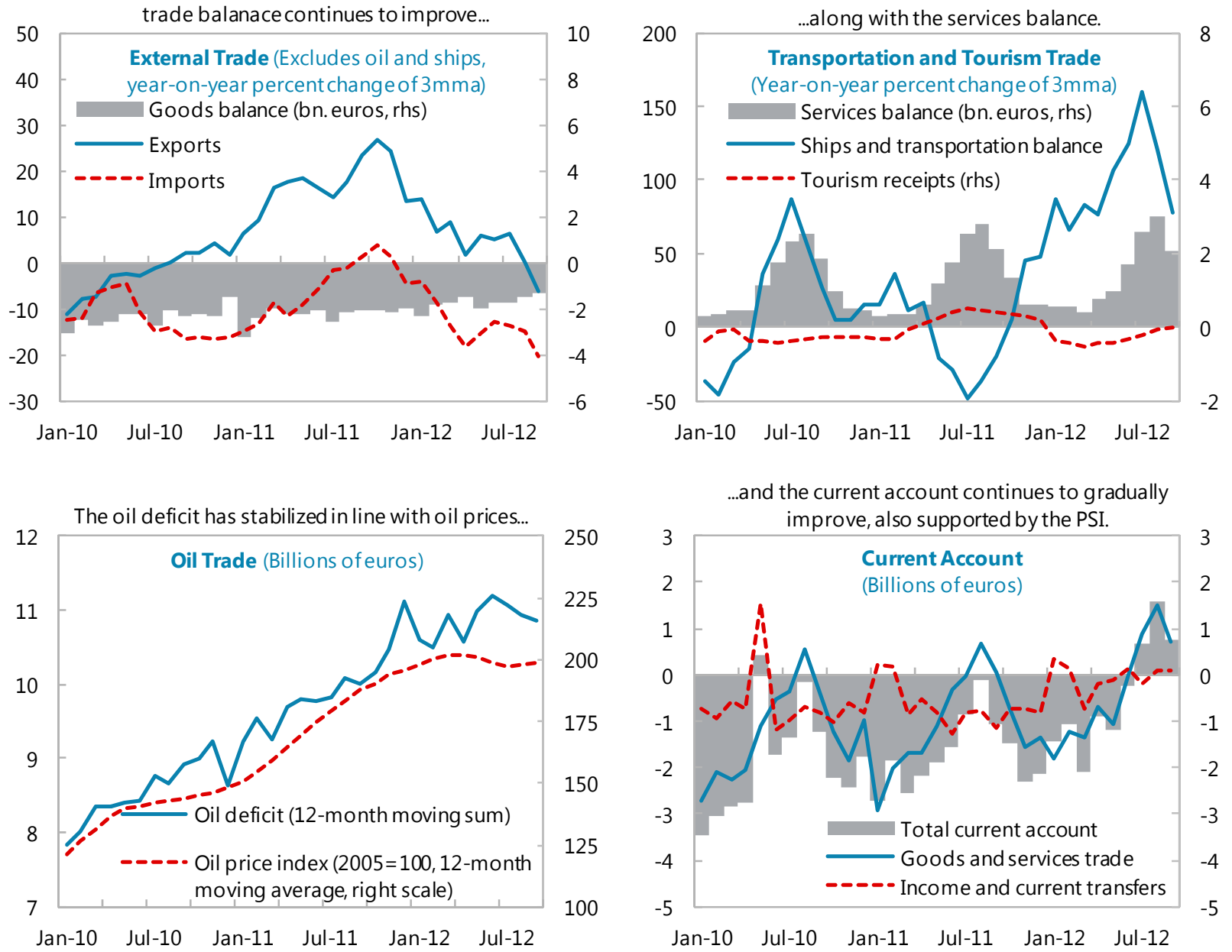

Sources: Bank of Greece; and IMF staff calculations. 


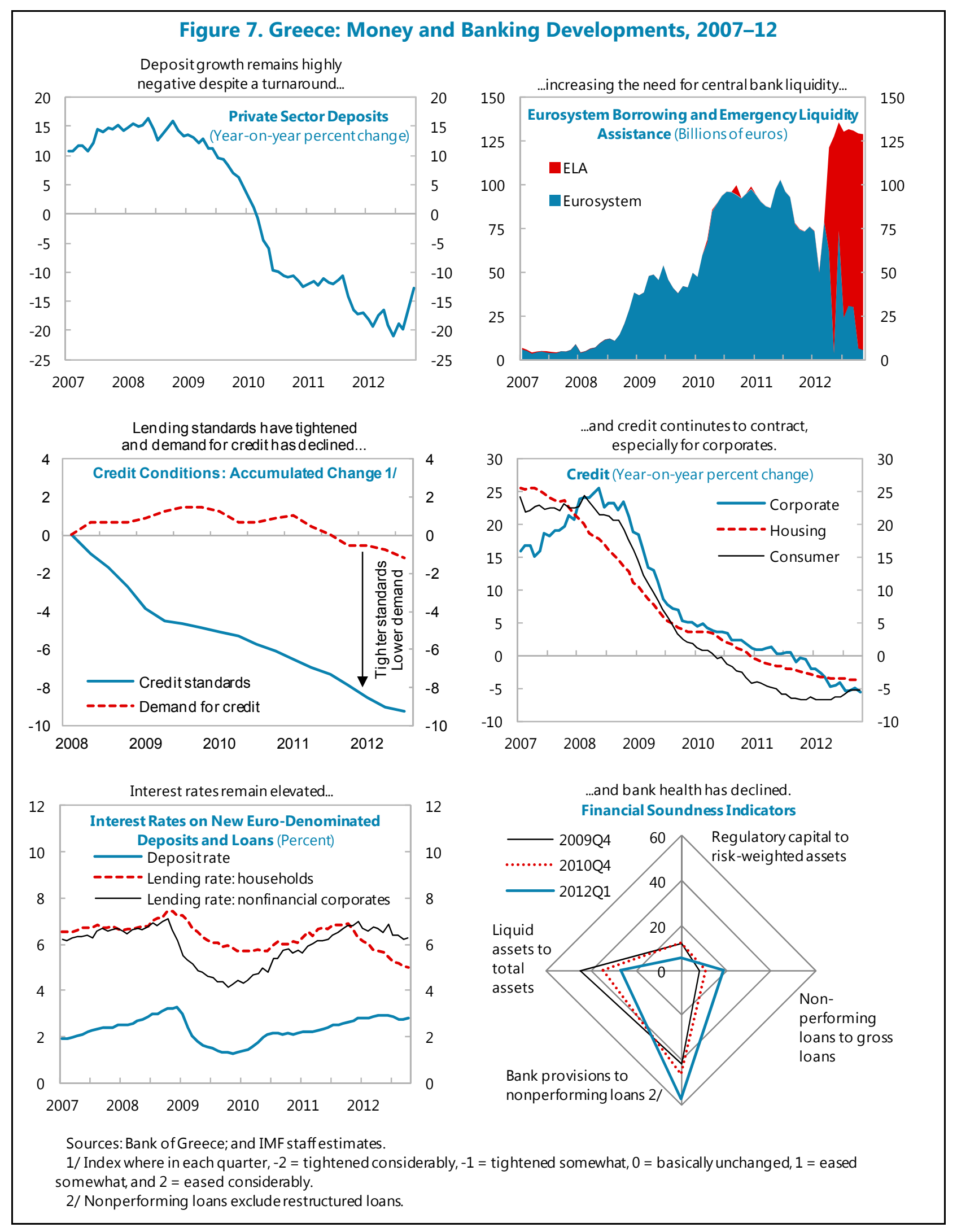




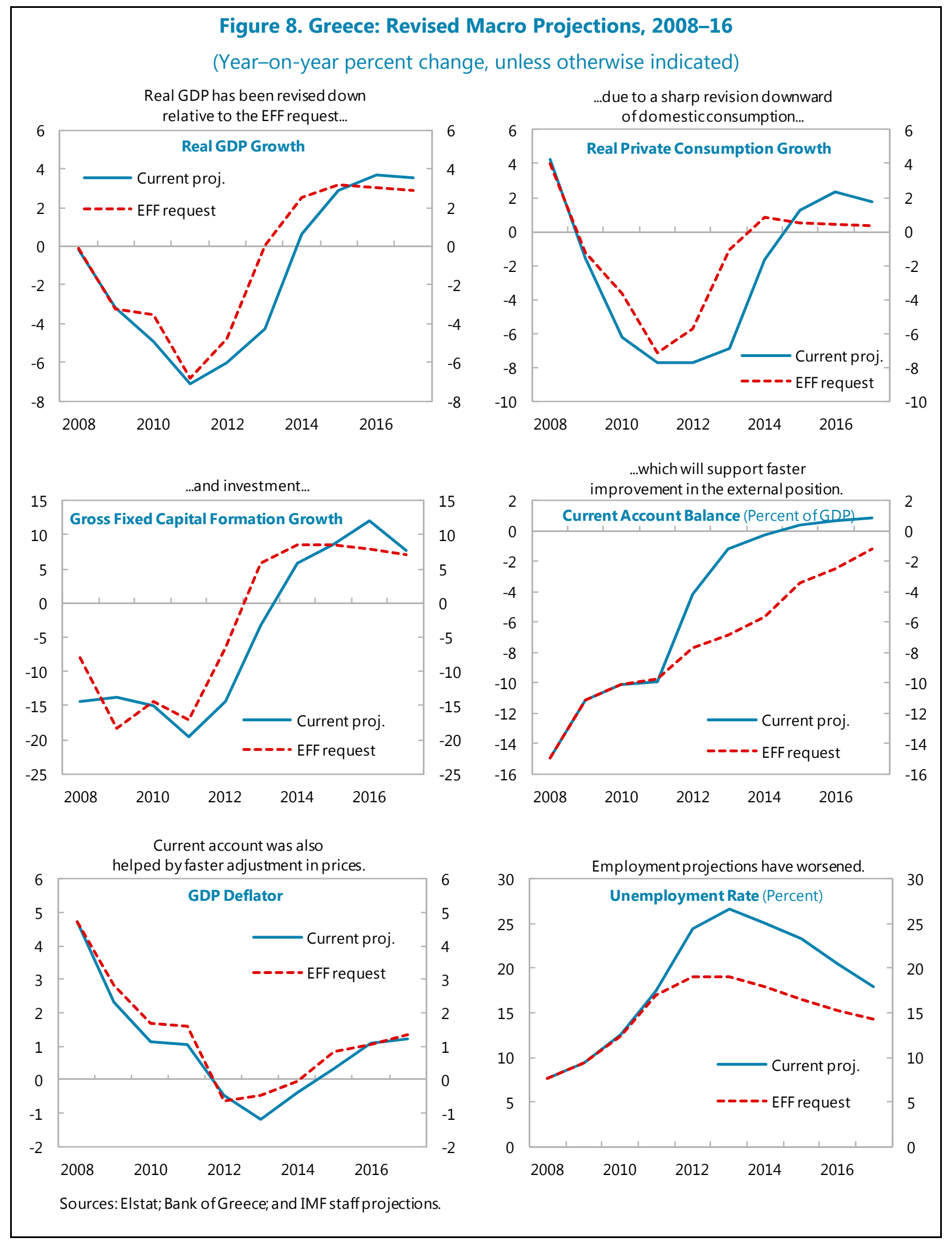




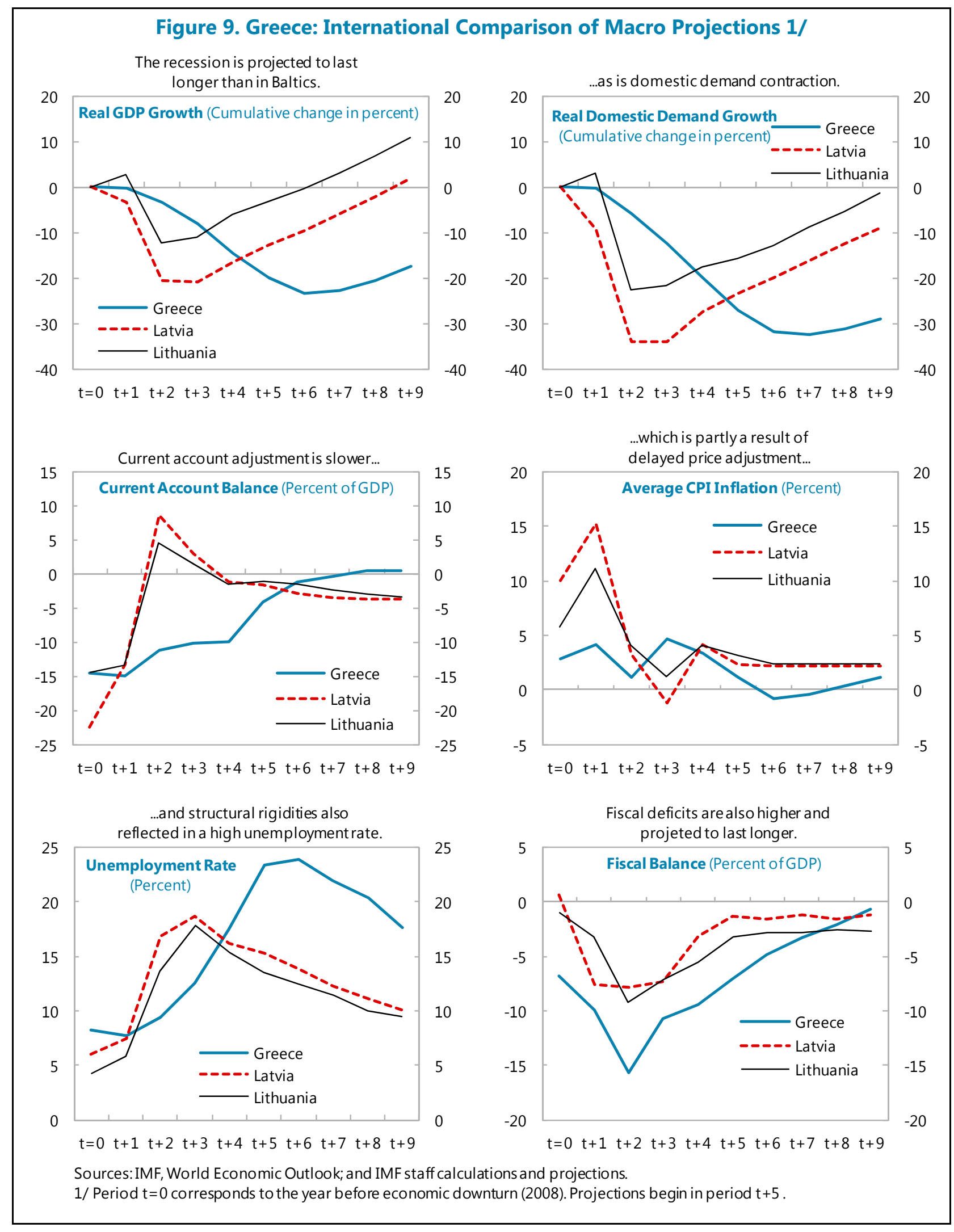


Figure 10. Greece: Financial Indicators, 2007-12
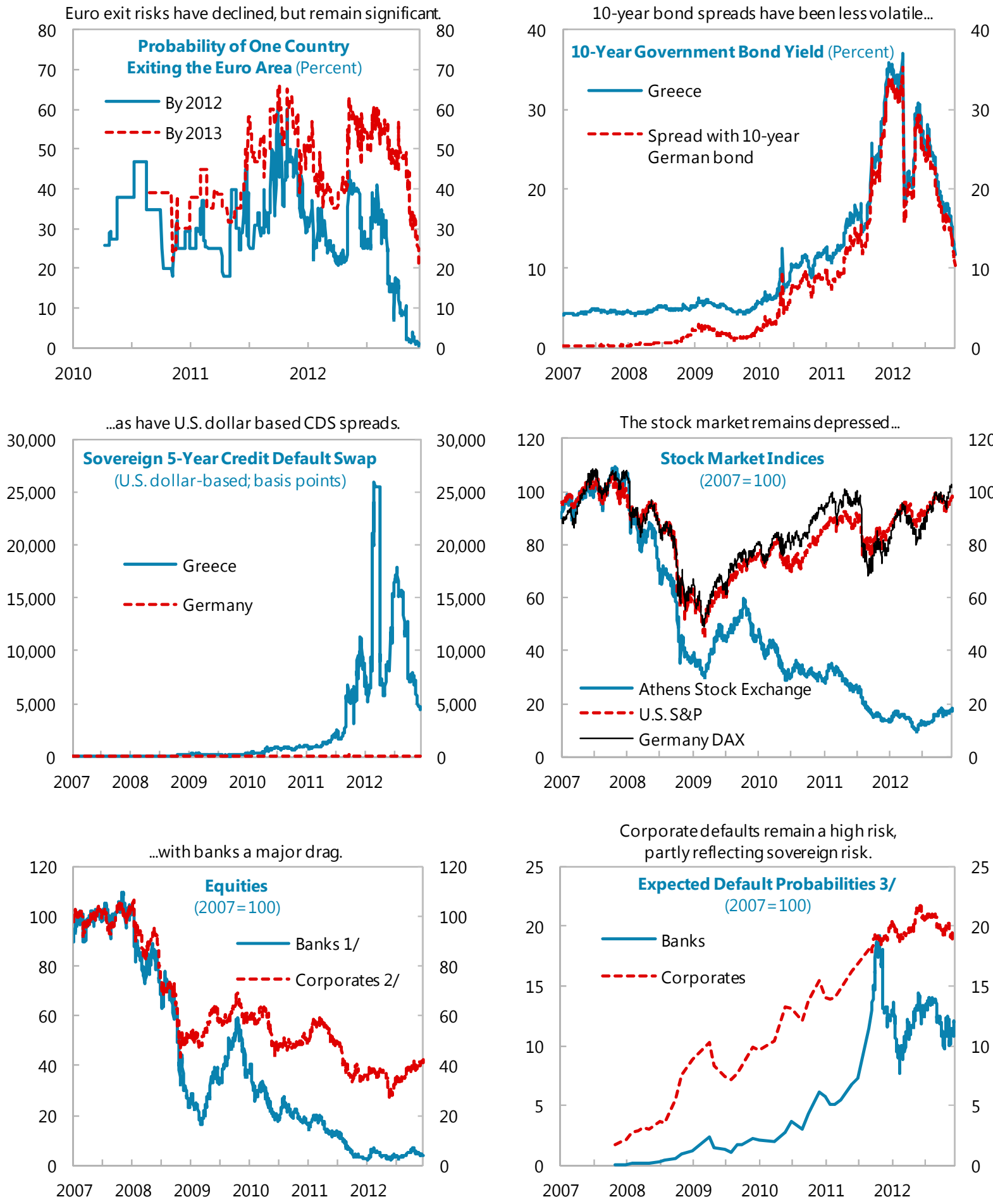

Sources: Bloomberg; InTrade; Moody's CreditEdge; and IMF staff calculations.

1/ Simple average of National Bank of Greece, Alpha Bank, EFG Eurobank, and Piraeus.

2/ Simple average of Coca-Cola Hellenic Bottling, Hellenic Petroleum, HellenicTelecom, OPAP, and Titan Cement.

3/ Current 1-year EDF calculated by Moody's Analytics. EDF is the probability of default where default is defined as the failure to make a scheduled debt payment. The EDF is driven by both asset volatility and market leverage. 
Figure 11. Greece: Revenue and Expenditure Trends, 2000-16

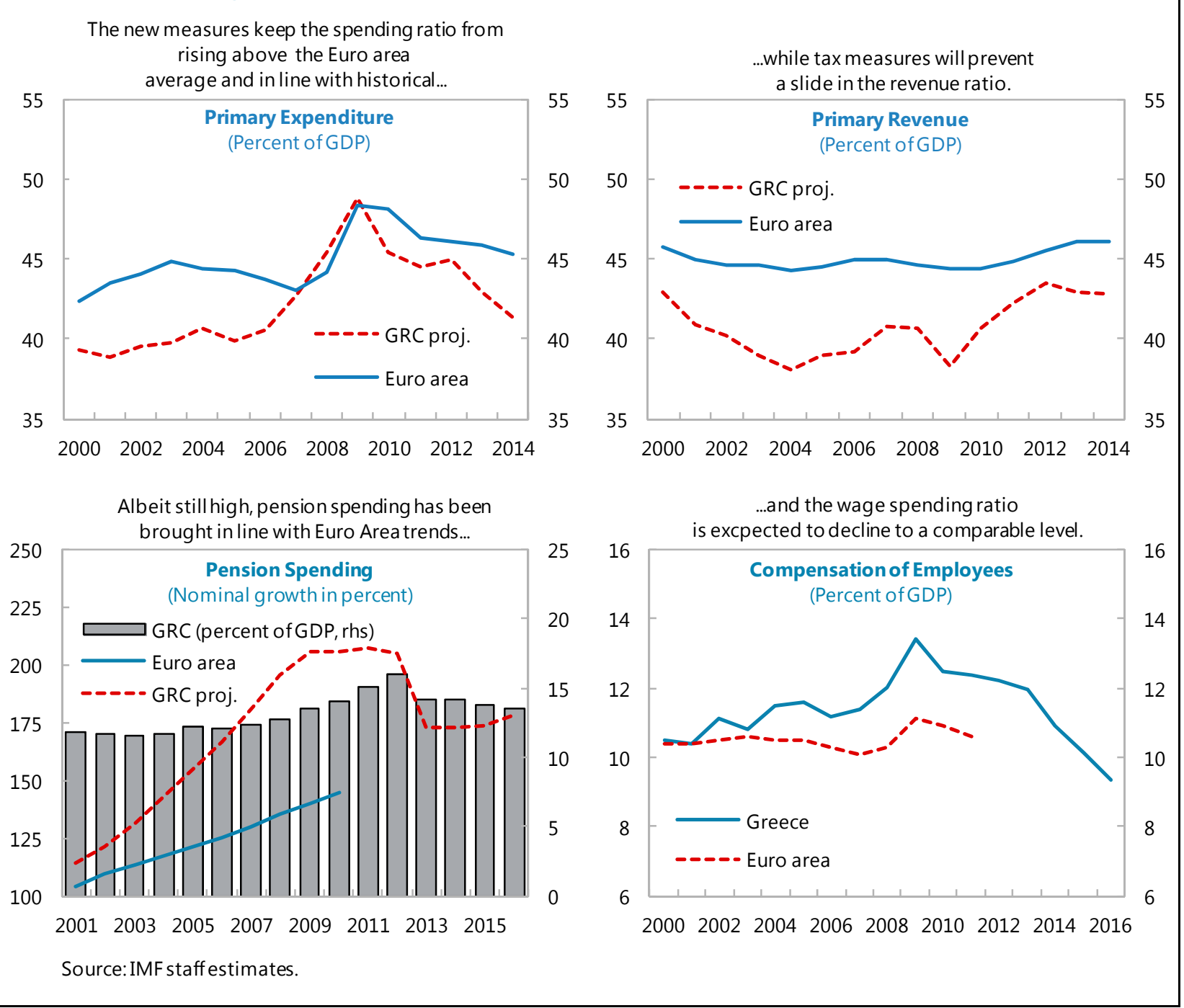




\section{Box 1. Increasing Revenue Collection: Progress and Bottlenecks}

Losses to the state from tax evasion are enormous. The shadow economy is estimated at 25 percent of GDP. Tax evasion is particularly common among the self-employed-doctors, lawyers, engineers, accountants. Empirical estimates suggest that the actual income of these taxpayers is between $1 \frac{1 / 4}{4}-2 \frac{1}{2}$ times their reported income (Artavanis et al, 2012), resulting in some 6 percent of GDP in unreported income and potential income tax losses of about $1-13 / 4$ percent of GDP in 2012. ${ }^{1}$ VAT fraud is also widespread, with 450,000 fictitious invoices detected since 2009. Tax debt reached $€ 53$ billion at end-September 2012.

Against this backdrop, the initial focus of reform efforts was on designing a strategy and a management approach. The focus was to be on five key areas: (i) large taxpayers; (ii) high-wealth individuals; (iii) tax arrears; (iv) filing enforcement; and (v) implementation of the government's new anti-evasion initiatives. Recognizing the limited implementation capacity of the tax administration, five task forces and a steering committee were established as a core project management arrangement.

In a second stage, work shifted to removing barriers to effective administration. A bill was enacted in late2010 to remove tax collection impediments, sanction tax fraud, establish a basis for addressing corruption and poor performance among staff, and streamline the judicial appeals process. The government also introduced ITbased risk analysis, an automated filing enforcement system, and centralized monitoring of high-risk taxpayers.

\section{However, implementation—the third stage—has been stalled for most of the last year:}

- Resources have not been redeployed. Task force plans were not translated into actions by local tax offices. For instance, despite annual uncollected net tax revenue at 86 percent of collections in Greece, against an OECD average of 12 percent, the administration does not devote sufficient staff to debt collection.

- New approaches have not been adopted. Managers - at headquarters and local offices-have not adopted new audit approaches and nor taken action on cases selected through risk analysis. Scarce resources have instead been devoted to low level verifications. The tax administration continues to focus on raising assessments-including fines with little chance of collection-but not on securing payments.

- Harmful practices have remained in place. The authorities have continued with generous amnesty-like installment arrangements, and have paid little attention to their design, increasing the workload in monitoring the schemes and frequently failing to do so past the first installment.

- New tools have not been adequately deployed. For instance, efforts to better use Greece's anti-money laundering framework have generated large numbers of suspicious transaction reports to the Financial Intelligence Unit, but this easily actionable information has received no priority in the tax administration. Also, in the judicial system, requirements for upfront payments to access an appeal have not been enforced.

\section{A critical reason for poor implementation has been inadequate organizational change:}

- Accountability has been lacking. Anti-corruption efforts have been minimal, and efforts to remove underperforming staff have met stiff resistance. Too little has been done to rein in powerful local offices. Legal frameworks that devolve all decision-making to local office heads have not been amended, and plans to reduce the network of local tax offices have been rephased twice. This has left the performance of core functions exposed to significant discretionary power by the heads of local tax offices.

- New recruitment has been inadequate. The authorities' commitment to recruit 1,000 audit staff by endApril 2012 was not met-only 140 auditors were hired. The plans for assessment and recruitment of 2,000 tax auditors over 2012 also collapsed. Existing employees have actively resisted new recruitment.

${ }^{1}$ Artavanis, Nikolaos T., Morse, Adair and Tsoutsoura, Margarita, Tax Evasion across Industries: Soft Credit Evidence from Greece (June 25, 2012). Chicago Booth Research Paper No. 12-25; Fama-Miller Working Paper. Available at SSRN: http://ssrn.com/abstract=2109500 or http://dx.doi.org/10.2139/ssrn.2109500. 


\section{Box 2. Greece as a Source of Contagion}

How strong are the contagion risks presented by Greece, and how have these evolved over time? Direct spillover risks have been receding, crisis preparedness in the euro area has improved greatly, and market proxies for contagion risk also suggest that risks have been falling. However, a key euro area crisis management measure- - the ECB's OMT facility (in conjunction with the ESM) - has not yet been tested. Moreover, market fears of default and euro exit remain (reflecting political uncertainties and difficult economic challenges), and the indirect spillover risks from a Greek exit remain substantial. This suggests that contagion remains an important concern.

\section{Direct spillover risks have been receding:}

- The exposure of the private sector to Greece is falling steeply, making direct financial losses from a potential default a less likely source of spillovers. The recovery rate on these claims would be low in case of exit and default since the output collapse combined with a sharp drop in the real exchange rate would weigh very heavily on Greece's capacity to service debt denominated in foreign currency. The impact of such losses would likely be small though: external liabilities to the private sector have fallen to less than one percent of euro area GDP. Similarly, trade linkages would likely play a minor role since Greece only accounts for 2 percent of euro area GDP, and most European countries' goods exports to Greece are less than one percent of their GDP (although it is in the 3-4 percent range in the cases of Bulgaria and Cyprus).

- The official sector's exposure to Greece through the GLF, the EFSF, and ECB and Eurosystem claims is increasing, but remains relatively small in percent of creditor countries' GDP. In case of exit, the write-down on GLF and EFSF loans would need to be deep to restore debt sustainability. Resolution of ECB and Eurosystem liabilities could impose significant losses on the Eurosystem and require member states to provide fiscal resources to recapitalize their national central banks. The impact of such contingencies on member states' public debt ratios would be relatively modest since their total exposure to Greece is around 2 percent of their GDP. However, the capacity of the EFSF/ESM to borrow could be affected or it could face higher borrowing costs.

- There are lingering risks in countries where Greek bank subsidiaries have significant market shares, but these are being managed. Greek subsidiaries account for 14 to 23 percent of the banking sectors in Albania, Bulgaria, FYROM, Romania, and Serbia, shares that have been broadly stable over time. Bulgarian and Romanian subsidiaries also rely significantly on parent funding (20 to 30 percent of their balance sheets). Policy actions have reduced the scope for spillovers, and the frequency of information sharing and coordination among national authorities has been increased to avoid the adoption of unproductive preemptive measures. However, banking relationships between Greece and Cyprus remain a notable potential source of spillovers. Branches of Cypriot banks in Greece are large (their loans to Greek residents amount to $€ 23$ billion or 130 percent of Cypriot GDP).

Market-based proxies of contagion risk suggest that the likelihood of spillovers in case of exit and default has fallen over time. The analysis focuses on market co-movement between Greece and a group of vulnerable euro area countries defined as those with CDS spreads above 500bps at some point since end2009 (Cyprus, Italy, Ireland, Portugal, Slovenia, and Spain). Rolling bond spread correlations fell sharply in 2011, although they have picked up moderately in 2012. Spikes in Greece CDS that do not coincide with CDS spikes in other vulnerable countries have become more common over time. A spike is defined as a change in daily CDS that is above the $90^{\text {th }}$ percentile of a country's distribution of changes in daily CDS. This suggests that it is increasingly common to see Greece-specific events that do not affect other countries. The simple statistics presented here should nevertheless be interpreted with caution: they do not imply causation, they are not robust to common shocks affecting both Greece and other vulnerable countries and, in the case of 
Box 2. Greece as a Source of Contagion (continued)

correlations, across-the-board spikes in volatility can bias the statistic upwards.
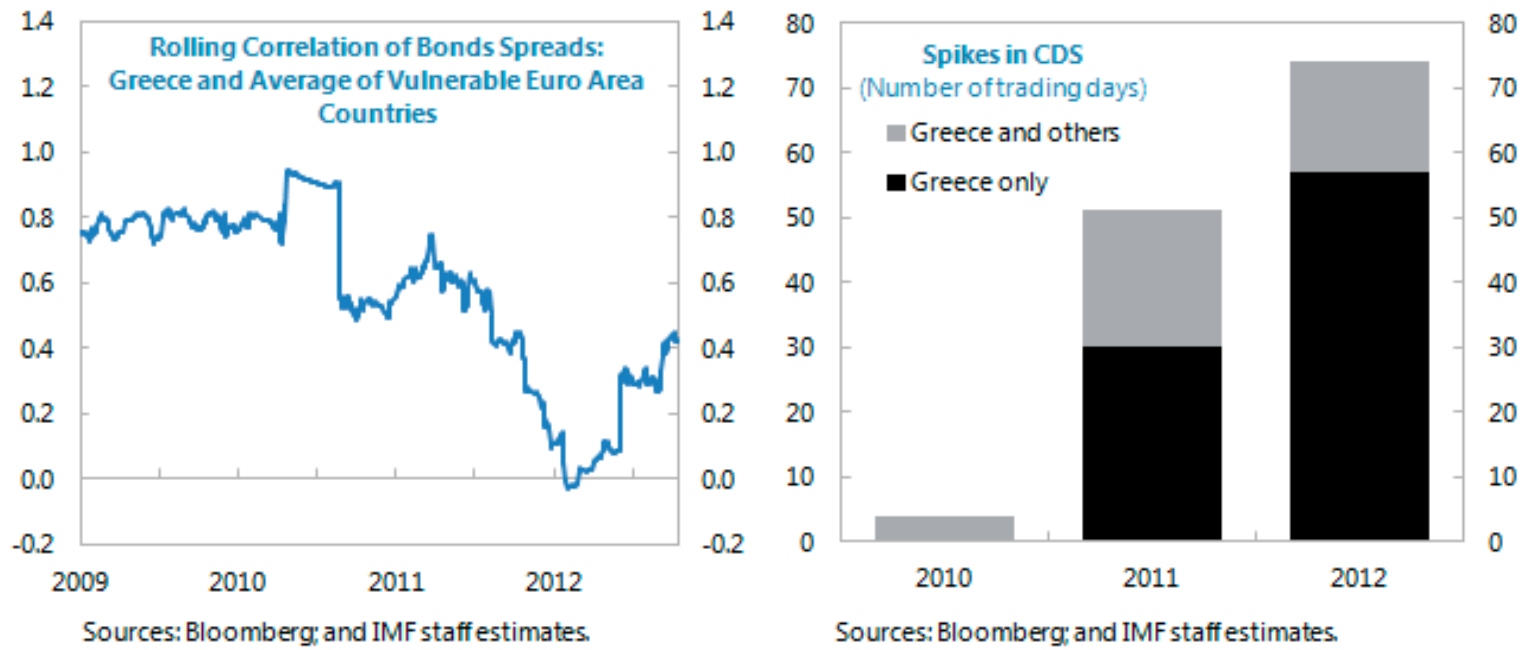

However, market fears of Greek exit from the euro remain elevated. The efforts of Greek and European leaders have helped, but have not taken this risk off the table. Indeed, until Greece completes its external adjustment and restores its competitiveness, it cannot be fully taken off the table.

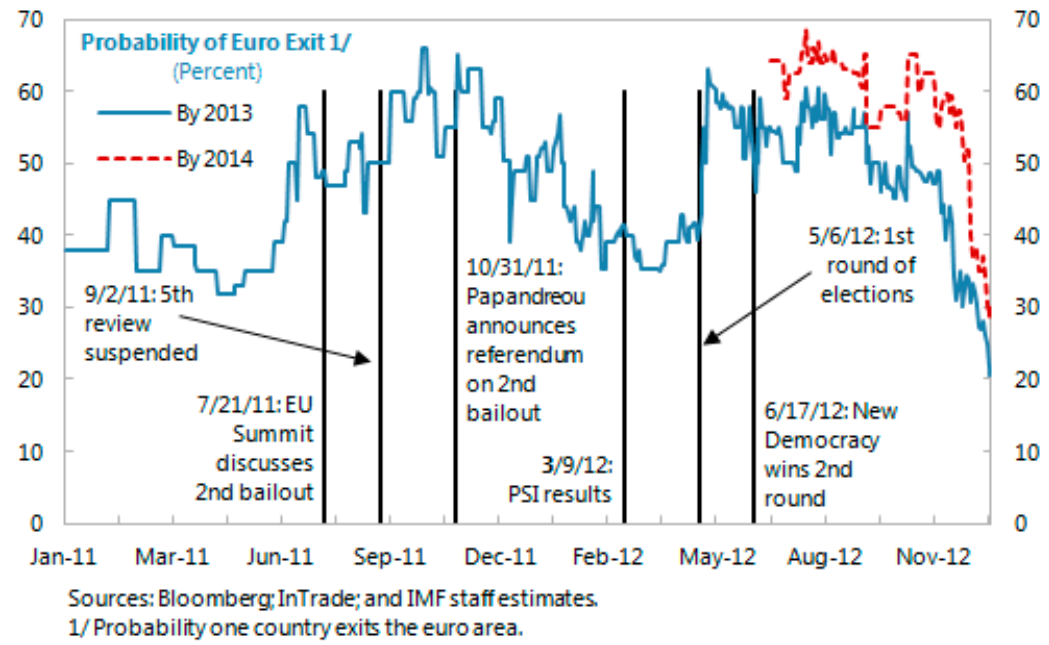




\section{Box 2. Greece as a Source of Contagion (continued)}

Despite the evidence of reduced scope for spillovers, the potential euro area output cost of a Greek exit is fundamentally uncertain and of a very large magnitude in certain scenarios. The historical relationship between financial stress and growth is a useful benchmark to guide a discussion of the potential real effects of euro exit. Financial distress is measured using the Financial Stress Index (FSI) in Cardarelli, Elekdag, and Lall (2011) augmented to explicitly capture sovereign risk. The relationship between the FSI and growth can be estimated for individual countries using a VAR model. The methodology does not model specific spillover channels, but it captures the average effect of all channels that have been at work in past distress episodes. The nature of the FSI shock that exit would trigger is key to derive the corresponding output implications and depends on factors such as the effectiveness of the European firewall and inherently unpredictable aspects like the possibility of bank and repo-market runs. If the FSI shock was similar to that

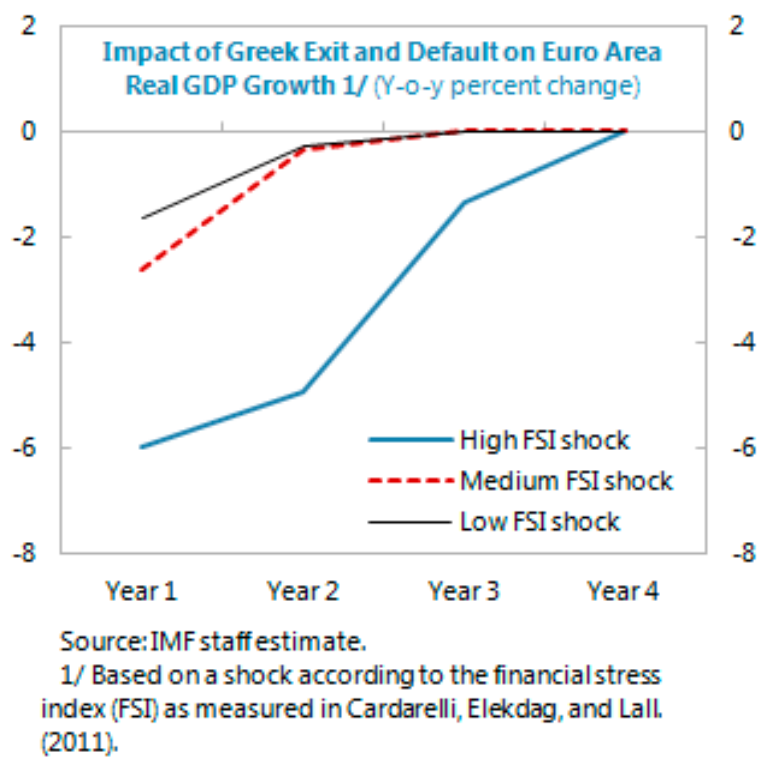
(2011). observed when LTCM collapsed in 1998, the methodology suggests that the euro area output loss could be around $1 \frac{1}{2}$ percent on impact. Modeling the shock based on the Scandinavian banking crisis of the early 1990s would yield a higher output loss of close to 3 percent. If Greek exit turned out to be a catastrophic event like the collapse of Lehman Brothers, the model indicates that the output losses could be very large and persistent. Falling exposure of the private sector to Greece and the stronger European firewall should make the latter scenario more unlikely, but it is not possible to establish that contagion risk from euro exit would be limited and manageable. 


\section{Box 3. The Macroeconomic Impact of a Debt Overhang}

Both theory and empirical evidence point to a strong link between debt levels and growth:

- A detrimental effect on investment and growth can in theory come through several channels:

$>$ Concerns about the government's ability to service the debt raises credit risk and interest rates, affecting investment, capital accumulation, and growth. The greater the uncertainty about prospects and policies is, the stronger is the effect on the risk premium. Extreme cases of a debt crisis can trigger a banking or currency crisis. Greece's public sector reliance on official financing reduces the impact of uncertainty, but private sector financing costs are affected, since corporate credit ratings have been strongly correlated with those of the sovereign (mainly because of the fear of repercussions from euro exit and default).

$>$ A high level of debt reduces incentives by borrowing countries to invest to

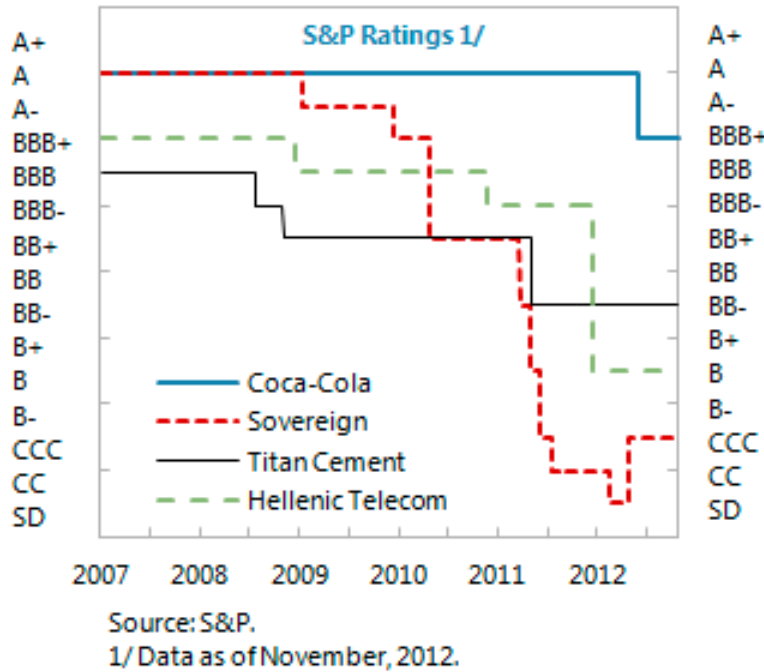
enhance growth prospects in the future. Growth can be affected by decisions at the micro level (firms reduce investment in anticipation of future increased taxation, expropriation through inflation, or dislocation costs related to sovereign default). It can also be affected at the macro level, through reduced incentives to implement growth-enhancing structural reforms when most of the benefits would accrue to creditors through debt service costs. In Greece, concerns about future taxation have been cited as affecting recent decisions by Greek companies to shift their headquarters out of the country.

$>\quad$ The necessary current account adjustment negatively affects growth in the short run. Large external liabilities ultimately require large trade surpluses in order to service them, and achieving these surpluses requires a more depreciated level of the real exchange rate. In a currency union the depreciation has to be achieved largely through deflation, which necessitates a larger negative output gap (though measures to increase flexibility of wages and prices can lessen this effect). For Greece, the reduction in the cost of debt servicing (through lower interest rates) and increasing the maturity of the debt will partly alleviate the short-term problem of resource transfers, but it is still there.

- There is a growing body of literature documenting the link between the level of debt and growth performance.

> The literature typically estimates a non-linear relationship between debt and growth and identifies thresholds beyond which debt and growth are negatively related. Among recent studies, Reinhart and Rogoff (2012) find that annual growth is over 1 percentage point lower when public debt ratio is above 90 percent in the long sample of advanced economies. Cechetti et al. (2011) estimate that growth is reduced by 0.13 percentage points for every 10 percentage points increase in the debt 


\section{Box 3. The Macroeconomic Impact of a Debt Overhang (continued)}

ratio beyond the 84 percent threshold in the sample of 18 OECD countries. Baum et al. (2012) show similar results for 12 euro area countries, showing a higher threshold (96 percent), but stronger negative effect on growth (-0.59 percent). Kumar and Woo (2010) report that a 10 percentage point increase in the debt-to-GDP ratio is associated with a slowdown in per capita growth of around 0.2 percentage points in the sample of emerging and advanced economies, with some evidence that higher debt has a larger negative effect on growth.

\begin{tabular}{|l|l|l|l|}
\hline Study & Sample & $\begin{array}{l}\text { Threshold beyond } \\
\text { which an increase } \\
\text { in public debt-to- } \\
\text { GDP reduces } \\
\text { growth }\end{array}$ & $\begin{array}{l}\text { Effect of a 10 percentage } \\
\text { points increase in public } \\
\text { debt-to-GDP on average } \\
\text { annual growth. }\end{array}$ \\
\hline $\begin{array}{l}\text { Cechetti, Mohanty, } \\
\text { and Zampolli (2011) }\end{array}$ & OECD economies & 84 percent & $\begin{array}{l}-0.13 \text { percentage points } \\
\text { (beyond threshold) }\end{array}$ \\
\hline $\begin{array}{l}\text { Baum, Checherita- } \\
\text { Westphal, and Rother } \\
(2012)\end{array}$ & $\begin{array}{l}\text { Euro area } \\
\text { economies }\end{array}$ & 96 percent & $\begin{array}{l}-0.59 \text { percentage points } \\
\text { (beyond threshold) }\end{array}$ \\
\hline $\begin{array}{l}\text { Kumar and Woo } \\
(2010)\end{array}$ & $\begin{array}{l}\text { Emerging and } \\
\text { advanced } \\
\text { economies }\end{array}$ & & -0.2 \\
\hline
\end{tabular}

There is also some evidence that growth tends to be higher after debt relief operations that make debt sustainable. Large restructurings (higher than the median reduction in the debt ratio in the sample from Das, Papaioannou and Trebesch (2011) and Cruces and Trebesch (2011) that eliminate the debt overhang are associated with an increase in median annual GDP growth by over 3 percentage points in the five years after the event.

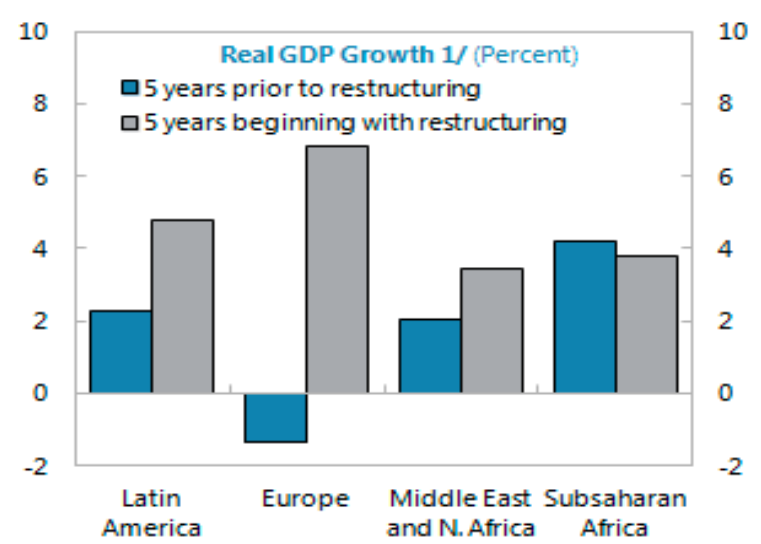

Sources:IMF, World Economic Outlook, and IMF staff estimates.

1/ Regional arithmetic average of annual geometric average. 


\section{Box 3. The Macroeconomic Impact of a Debt Overhang (continued)}

\section{References}

Baum, Anja, Checherita-Westphal, Christina and Philipp Rother, 2012, "Debt and Growth: New Evidence for the Euro area."

Cechetti Stephen, Mohanty, MS and Fabrizio Zampolli, 2012, "The Real Effects of Debt", mimeo Bank for International Settlements.

Kumar, Manmohan and Jaejoon Woo (2010), "Public Debt and Growth," IMF Working Papers, No. 10/174, International Monetary Fund.

Das, Udaibir, Papaioannou, Michael and Christoph Trebesch (2012), "Sovereign Debt Restructurings 19502010: Literature Survey, Data, and Stylized Facts," IMF Working Paper 12/203, International Monetary Fund.

Cruces, Juan J. and Trebesch, Christoph, "Sovereign Defaults: The Price of Haircuts" (October 13, 2011). CESifo Working Paper Series No. 3604 


\section{Box 4: Measures Taken to Address the Greek Debt Burden}

On November 26, euro area member states (EAMS) agreed on measures to reduce Greece's debt burden:

- Debt buyback: EFSF funding, within the existing program envelope, was made available for Greece to repurchase a portion of the $€ 61.8$ billion new Greek government bonds (GGBs) that were issued as part of the March 2012 PSI operation. These bonds were trading at a deep discount at mid-year but had recovered to 30 in the fall, on the back of improving prospects for concluding the first and second program reviews and public discussions about buybacks. The exchange was conducted via a Dutch auction, whereby maximum and minimum prices were set for each maturity (with a higher price range for the shorter maturities) and bidding took place in between. The buyback was completed in early December and retired $€ 31.8$ billion in exchanged bonds, including $€ 14.1$ billion from Greek banks. The average price reached 33.8 , with the tendered bonds spread about equally across the maturities. In all, the exchange is estimated to have reduced 2020 debt to GDP by $9 \frac{1}{2} 2$ percentage points.

- Lower interest rates on GLF and EFSF loans: The margin over 3-month Euribor on the $€ 53$ billion in debt to the Greece Loan Facility (GLF) was reduced from 150bps to 50ps, and the 10bps operating fee on EFSF lending was eliminated. The GLF reduction brings it below the market funding cost of some euro area member states, implying an effective fiscal transfer from countries facing larger effective sovereign borrowing spreads. The change in borrowing terms is estimated to alleviate the debt burden over time achieving a cumulative debt reduction by 2020 of approximately 2.6 percentage points of GDP.

- Eurosystem Profits: The EAMs agreed to remit profits on Greek government bonds purchased by the European Central Bank under the Securities Market Program (SMP). These profits resulted from the ECB buying the bonds at a discount to par and the lower cost of ECB funding relative to the interest rate on the bonds. Subject to approval by member country parliaments, Greece will receive about $€ 9.3$ billion, reducing 2020 debt by 4.6 percent of GDP with the proceeds accruing over time (starting in 2013). In some cases, this may require annual parliamentary approval.

Unspecified Measures: Member states agreed to take additional steps in 2014 and 2015-tied to Greece's progress with improving its primary budget balance-to reduce debt to Impact of Debt-Reducing Measures in 2020 (Percent of GDP)

\begin{tabular}{lr}
\hline Old baseline & 144.0 \\
Impact of: & -10.0 \\
Debt buyback & -2.0 \\
Interest rate reduction & -0.6 \\
Cancellation of EFSF fee & -4.6 \\
Remittance of SMP profits & -2.8 \\
To be specified & 124.0 \\
\hline
\end{tabular}

Source: IMF staff estimates. 124 percent of GDP in 2020 and to a level "substantially below" 110 percent of GDP by 2022. Options announced by member states include further GLF interest rate reductions and revisions to the framework for structural funds for Greece. On current estimates, measures delivering roughly 4.1 percent of GDP by 2020 will be needed to bring debt to 124 percent of GDP by 2020. Significantly more than that will be needed to deliver it substantially below 110 by 2022 (present projections show debt at 113 in 2022, even assuming that the 4.1 percent of additional relief is decided), which in all likelihood will necessitate either large reductions in EFSF interest rates or principal haircuts on the GLF. 


\section{APPENDIX I. DEBT SUSTAINABILITY ANALYSIS}

1. This appendix considers the sustainability of Greece's public and external debt. Section A provides an overview of the macro, policy, and financing inputs underpinning the revised program macro framework. Section B considers public debt sustainability, examining the debt trajectory under the program baseline, and under a variety of stress scenarios (to illuminate how the debt trajectory would evolve if macro, policy, and financing assumptions were to differ, either individually, or jointly). Section $C$ considers external debt sustainability, again under baseline and stress scenarios. Results show that the revised program can place Greek debt on a sustainable trajectory. There are significant risks that debt declines may be interrupted or even reversed by shocks, but maturity extension and lower interest rates from official creditors have improved robustness to shocks.

\section{A. Key Inputs for the DSA}

2. The macro and policy frameworks underlying the DSA have been updated. The key changes relative to the framework in the March 2012 program include:

- A lower output path. The macroeconomic outlook is projected to be less favorable than assumed at the outset of the program. Political uncertainty, implementation risks, and continued euro exit fears are expected to suppress investment for some time, affecting both demand and potential output. The external environment for Greece has also worsened, as the euro area has lurched towards a deeper recession. These two factors are expected to delay the recovery yet again. Meanwhile, the overall path for nominal income has also been affected by recent national accounts revisions (which removed almost [5] percent from nominal GDP).

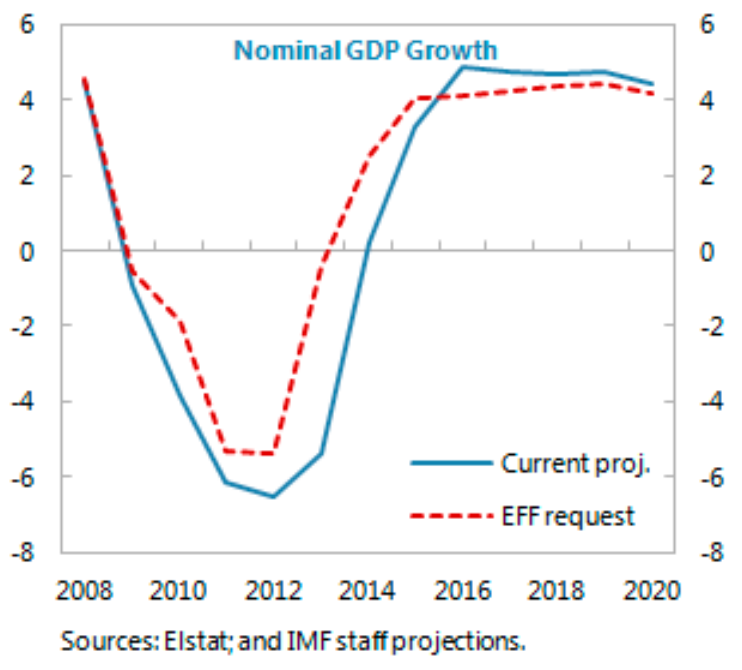

- Weaker external conditions. External demand by Greece's trading partners is expected to recover more slowly than projected in the program, 13/4 percentage points lower in 2013 and $3 / 4$ percentage points lower in 2014. It would then gradually converge to the previous forecast. Regarding the terms of trade, these are forecast to be close to program projections for 2013, and improve in the medium term compared to the program on the back of lower energy prices. Finally, measured in unit labor cost terms, the real effective exchange rate overvaluation now is expected to disappear sooner than projected in the program (2014 vs. 2015). 
- Slower fiscal adjustment. The fiscal adjustment period has been extended by two years, with a primary surplus of $4 \frac{1}{2}$ percent of GDP now reached in 2016. The assumed long-term primary surplus of 4 percent of GDP remains the same. As noted in the DSA for the March 2012 program, this is ambitious but feasible when taking into account the experience of other countries. In the euro area, Italy managed to run an average primary surplus of about 4 percent of GDP over a period of a little longer than a decade. Belgium has managed to post average 10-year primary surpluses as high as $5 \frac{1}{2} 2$ percent of GDP.

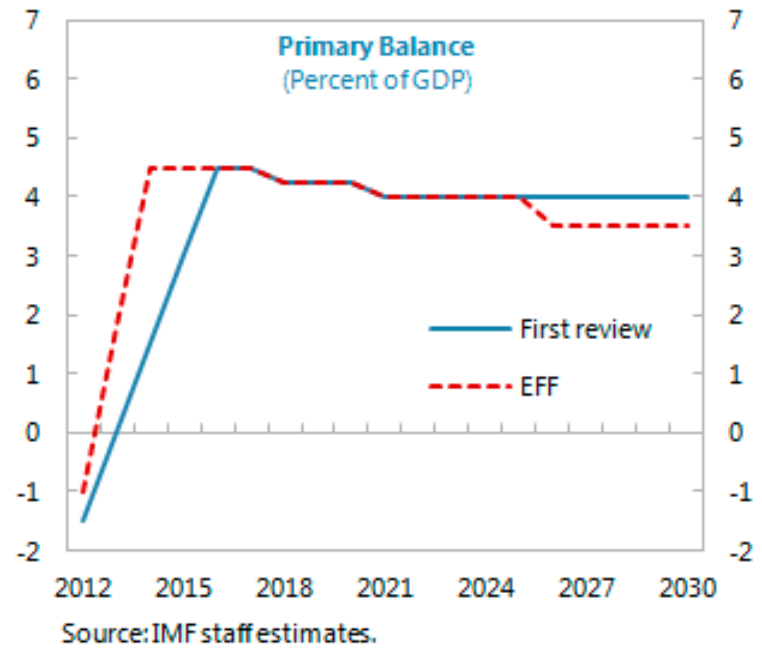

Lower privatization proceeds. Expected proceeds from privatization for $2012-20$ have been revised downwards from $€ 45$ billion to $€ 22$ billion. With annual proceeds still sustained in the $1-1 \frac{1}{2}$ percent of GDP range for several years, this target will also be challenging, but remains within the range of international experience. There are three key reasons behind the revision: (i) heightened implementation risks and more notable euro exit fears are expected to depress demand for privatized assets, weighing on sales volumes and prices; (ii) an updated bottom-up assessment suggests that fewer real estate assets are

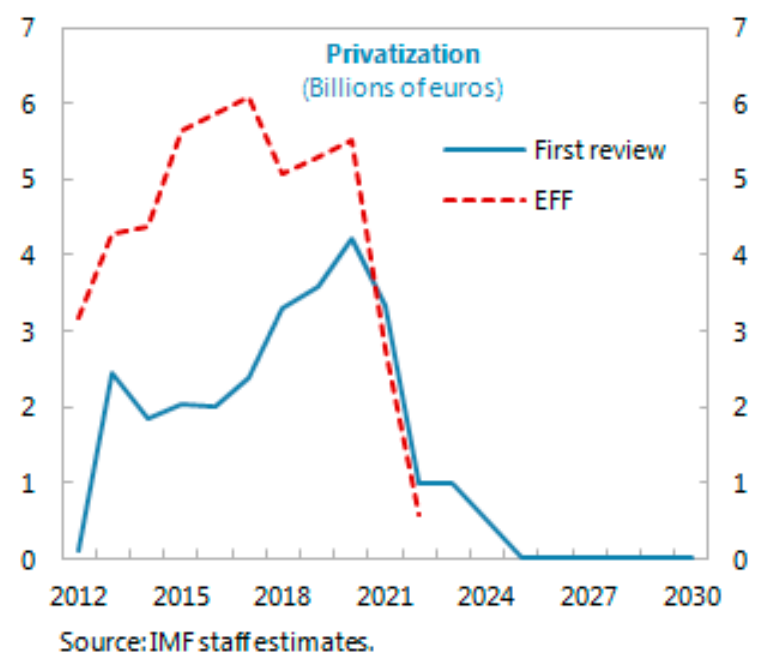
good prospects for sales (reflected in the authorities' own forecasts for proceeds); and (iii) in light of more evident political constraints, which come in addition to now more-visible regulatory and judicial barriers, the process for bringing assets to the point of sale are likely to be longer and more drawn out.

Other adjustments. A total amount of $€ 8$ billion in arrears is assumed to be cleared in 2012-13, $€ 2$ billion higher than projected at the outset of the program. Meanwhile, the pace of government deposit accumulation has been reduced relative to the EFF ( $€ 1.5$ billion in deposit accumulation will be targeted by 2014 as opposed to $€ 5$ billion; with deposits returning to the program path thereafter). Also, the path for the stock of T-bills has been modified to allow the authorities to maintain a stock of $€ 15$ billion over the program period rather than reducing this stock to $€ 6$ billion as foreseen in the EFF. Finally, the estimated 
cost of financial system support has been reduced from $€ 50$ billion to $€ 49.4$ billion (see $\llbracket 31$ in the staff report).

3. The framework for program financing has also been updated. At the Eurogroup meeting on November 27, 2012, euro area member states adopted a number of concrete measures to assist Greece, and promised further conditional assistance once Greece reaches an annual primary surplus, to help Greece reach a debt to GDP level of 124 percent in 2020 and substantially below 110 percent by 2022 . The DSA does not attempt to model the commitment to bring debt below 110 percent, but does reflect the to-be-specified measures to achieve 124 percent of GDP in 2020:

- Revised terms and conditions for official financing. In line with decisions taken, the DSA assumes: (i) a reduced interest rate on the Greek Loan Facility (from a margin of 150 bps to a margin of 50 bps over Euribor); (ii) cancellation of the 10 bps administrative fee on EFSF loans; (iii) receipt of profits on the ECB's SMP portfolio (a projected flow of approximately $€ 9.3$ billion through 2020); and (iv) a deferral (and capitalization) of interest payments on EFSF and future ESM loans until 2022. The Eurogroup committed to adopt an additional 4 percent of GDP in debt reduction measures after 2014, conditional on Greece achieving a primary surplus. This is modeled as a constant fiscal transfer after the program period.

- An upfront reduction in debt through a buy-back of Greek government bonds. A voluntary debt buyback from the private sector was completed by the authorities on December 18 , 2012. In total, $€ 31.8$ billion of bonds were retired at the cost of $€ 10.8$ billion. Immediate financing to complete this operation was provided via EFSF support (within its existing arrangement).

\section{Market access assumptions have also been updated, and the DSA has been extended} to 2030, to evaluate how higher debt ratios may affect Greece's ability to refinance debt coming due. Small but building amounts of market borrowing are assumed to begin in 2018, culminating in full reliance on the market after 2020. For modeling purposes, it is assumed that the terms of borrowing are directly linked to the level of Greece's debt. At a debt level of roughly 115 percent of GDP, Greece is assumed to be able to borrow at a spread of 450-600 bps depending on the maturity (reflecting current spreads for high-debt European countries plus a premium for Greece's recent history of debt restructuring) with spreads rising by 10 bps for every 1 percentage point increase in debt. These assumptions imply entry into the market at relatively high spreads and shorter maturities, with improvements over time as debt falls. 


\section{B. Public Sector DSA}

\section{Baseline projections show that debt would decline to 124 percent of GDP in 2020 and}

to 113 percent by 2022 (Table 1). The 2020 figure is significantly higher than the $1161 / 2$ percent of GDP projected at the outset of the EFF-supported program. Moreover, debt is expected to peak near 180 percent of GDP in the near term, up from the previous projected peak of 167 percent of GDP in the original program forecast. Steady reductions in the debt ratio commence in 2014, albeit at higher levels than in the original program forecast. The debt ratio is projected to drop below 100 percent of GDP in 2026, one year later than in the original program. To the extent that Europe does take measures to reduce Greece's debt substantially

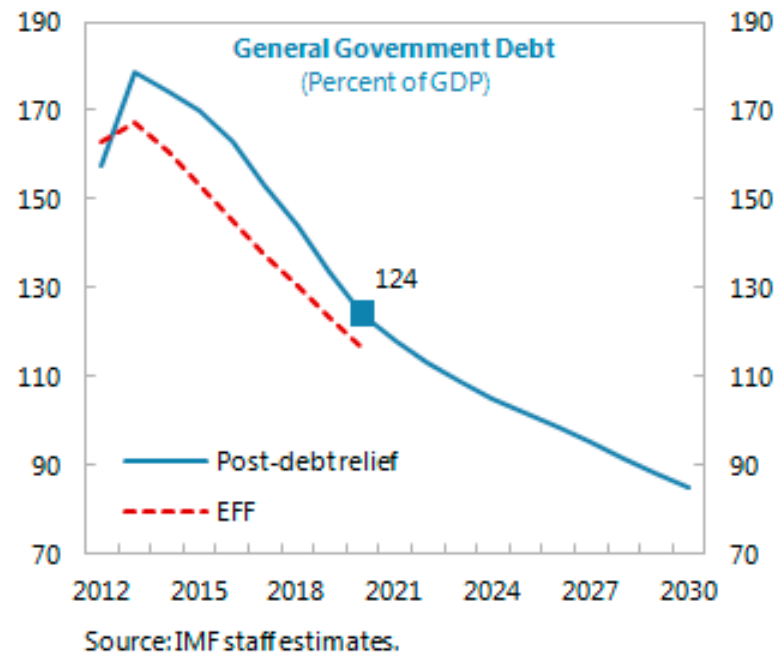
below 110 by 2022, remaining deviations relative to the program would be corrected (under the program, debt was projected to fall to 107 percent of GDP by 2022).

\section{Despite the higher debt levels than under the Extended Arrangement request, the debt} service burden is considerably lower over the next two decades due to the debt relief

measures. The interest rate reductions and deferrals reduce interest payments by up to 3 percent of GDP per year. Meanwhile, the maturity extensions on EFSF and GLF debt sharply reduce the amount of debt that matures starting in the early 2020s. This will be important in mitigating the refinancing risk that Greece would otherwise face if it had to go to the markets to refinance large amounts of maturing official debt at a time when its public debt levels were still very high.

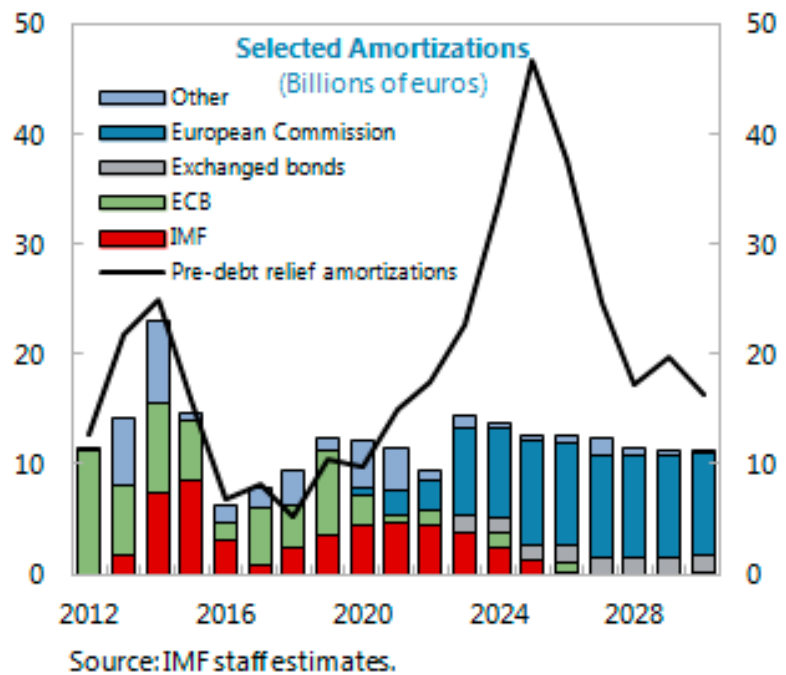

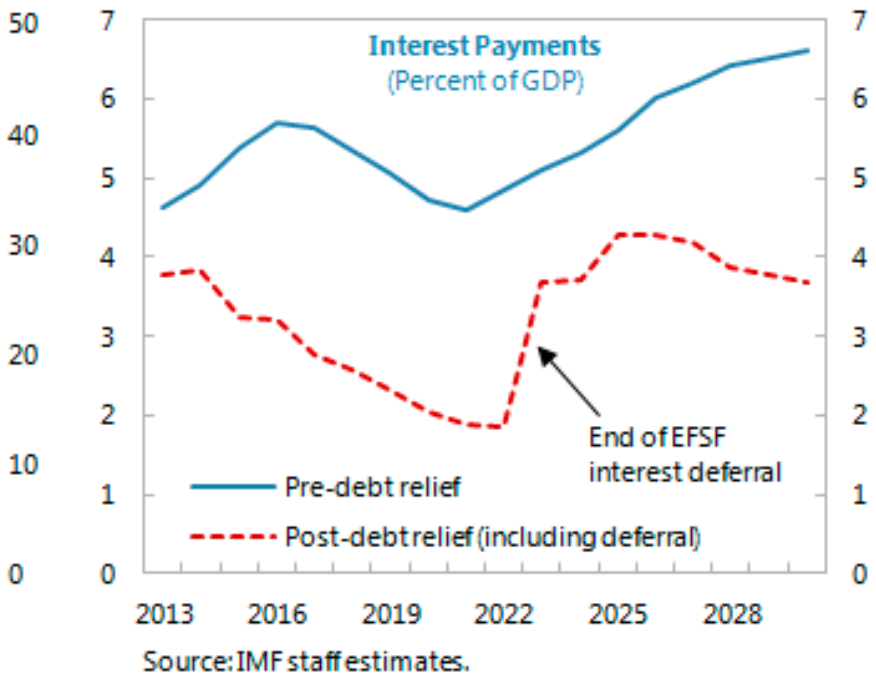




\section{Stress tests point to continued sensitivities, but the profile of risks is more resilient as a result of the debt reduction measures:}

- Policies. If the primary balance gets stuck at a surplus of $1 \frac{1 / 2}{2}$ percent of GDP (a level it will reach in 2014), then debt would flatten out above 125 percent of GDP over the medium term; a lower primary result would put debt on an ever-increasing trajectory Significant shortfalls in privatization proceeds (only half of the $€ 21$ billion projected by 2020), would raise the level of debt by about 5 percentage points of GDP but preserve a downward trajectory. Post debt relief measures, resilience of the debt trajectory is broadly similar to that identified under the Extended Arrangement.
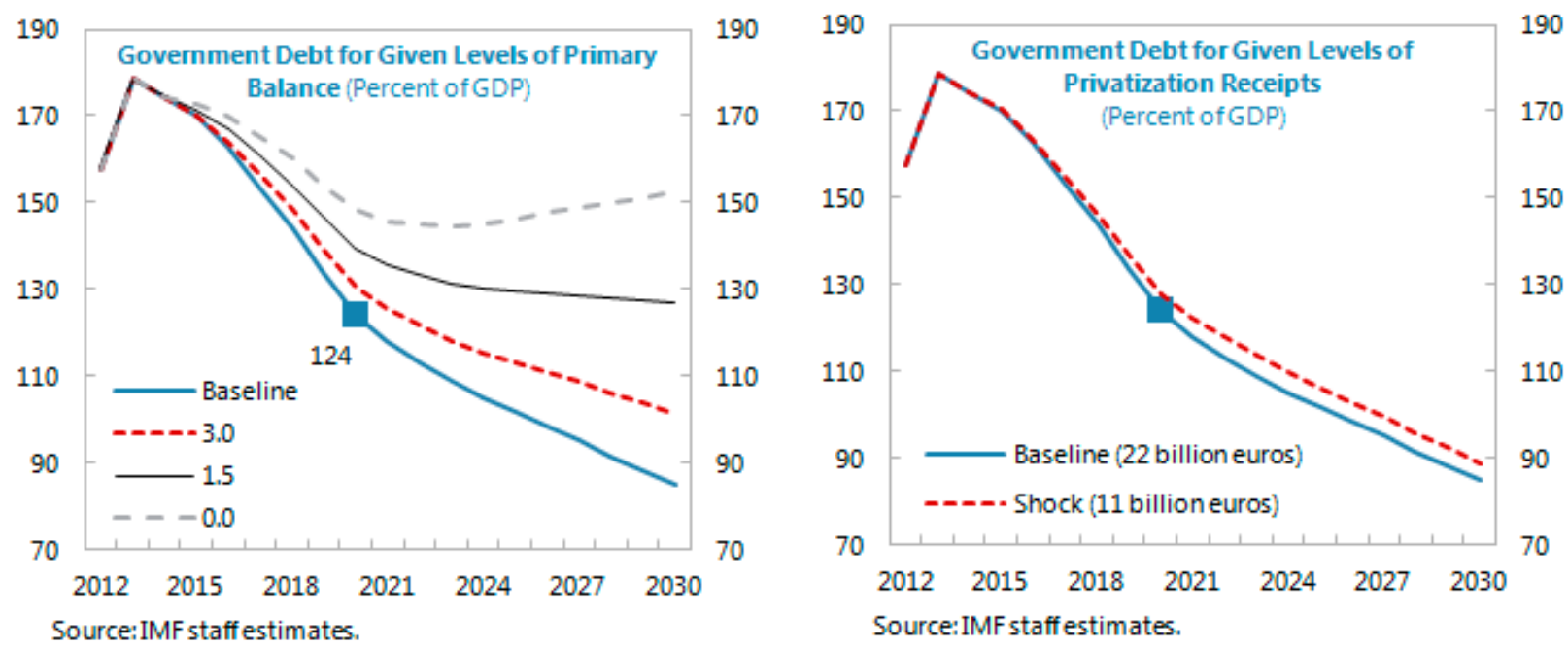

- Macro parameters. Debt outcomes also remain sensitive to growth or variations in the pace of internal devaluation. Fixing the primary balance path, nominal growth permanently lower by 1 percent per annum would increase debt-to-GDP to 136 percent by 2020. Nominal growth permanently higher by 1 percent per annum would allow debt to fall to 105 percent of GDP by 2020. This sensitivity to growth shocks is broadly similar to that in the original EFF-supported program once the higher baseline debt level is accounted for. 


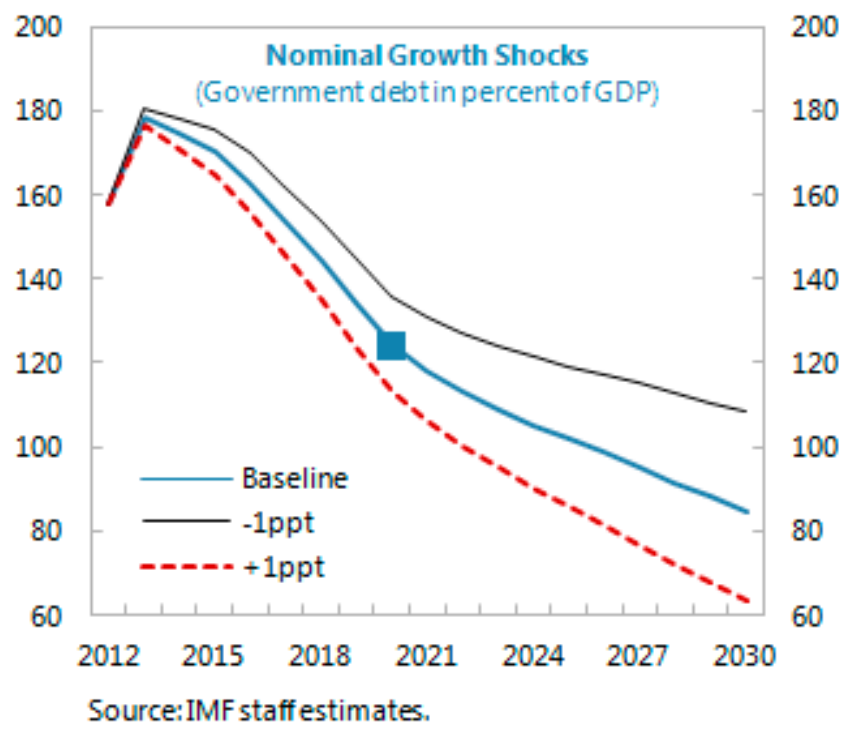

- $\quad$ Financing assumptions. Interest rate sensitivities arise via the rate charged on official financing, and via interest on new market borrowing once Greece re-enters markets (primarily after 2020). If the spread on EFSF borrowing were to be 100 bps higher, then debtto-GDP would reach 130 percent by 2020. One key improvement to the resilience of Greek debt regards shocks to market interest rates. Given the extension of maturities on official sector debt, reliance on market access is adequately low that even a $400 \mathrm{bps}$ shock to Greek spreads over 2021-30 has a relatively modest impact on debt levels.

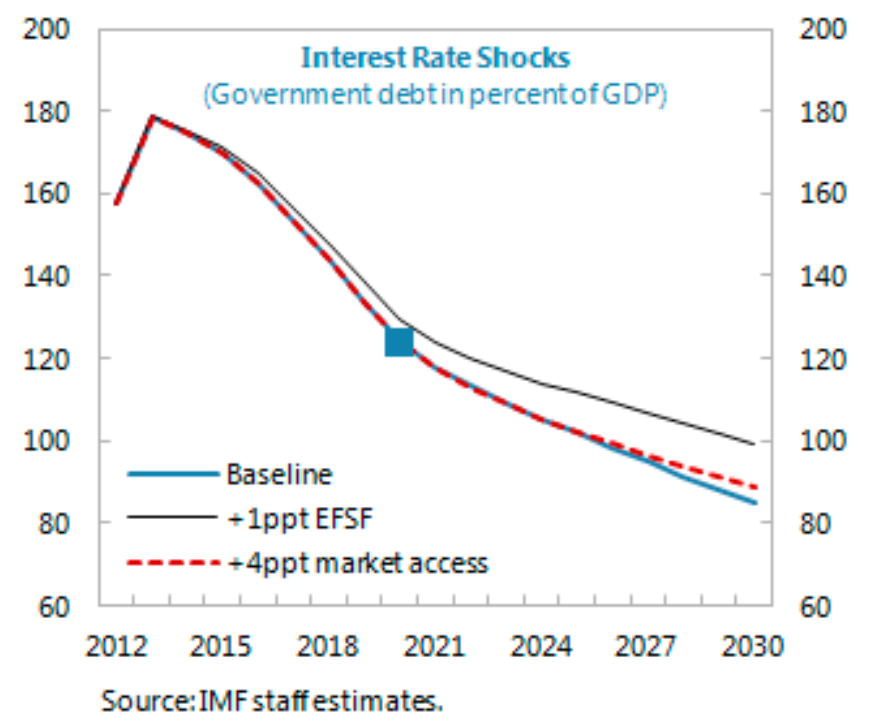

8. Two alternative scenarios are considered to further illuminate risks. These consider a combined shock (to capture the impact of program implementation delays), and a euro exit shock (to capture the potential impact of an intensified political crisis in Greece): 
The debt trajectory remains extremely sensitive to program delays, and this could bring sustainability into question absent further debt relief. This scenario assumes that a recovery in confidence and investment is delayed, structural reforms take longer to implement, and the economy continues to adjust through recessionary channels. On the policy side, it assumes that fiscal adjustment takes two years longer to reach the end-point target, and privatization delivers $€ 10$ billion less than targeted. In this scenario, the debt ratio would continue to peak at 180 percent of GDP but would remain higher for longer, falling to around 147 percent of GDP by 2020, some 23 points higher than under the baseline.

Thereafter the debt ratio would maintain its downward trajectory, but at significantly higher levels. With debt ratios so high in the next decade in this scenario, even small further shocks could produce unsustainable dynamics.

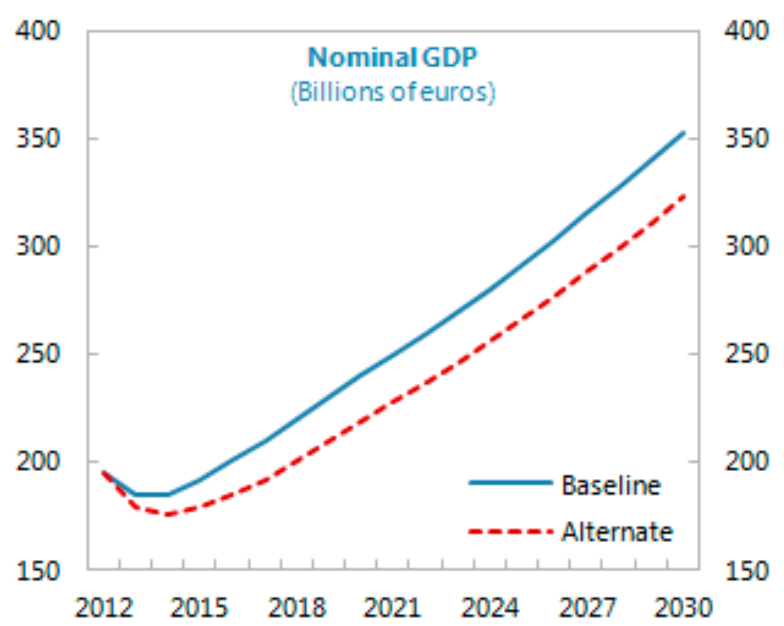

Source:IMF staff estimates.

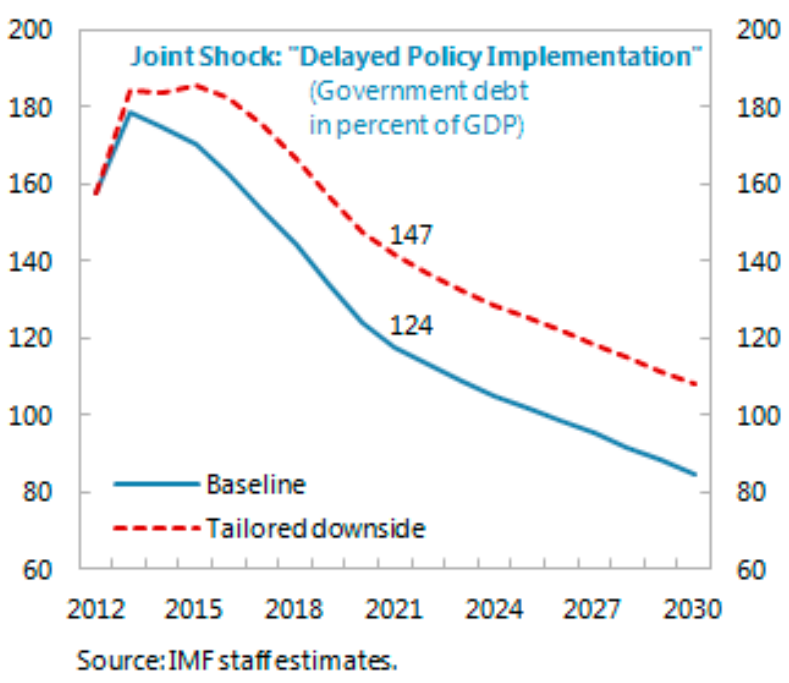

- If Greece were to exit the euro, debt would be unsustainable. Euro exit would be accompanied by a sharp depreciation of the new currency, as well as by a deep economic contraction (see Box 2 of the Extended Arrangement request staff report). Both these factors would act to reduce Greek GDP in euro terms, which could cause debt ratios to double to clearly unsustainable levels. The government would also likely need to absorb significant bank liabilities, which would add to debt ratios. To the extent that liabilities to the Eurosystem were fiscalized, this would add even further to the post-exit debt burden. 


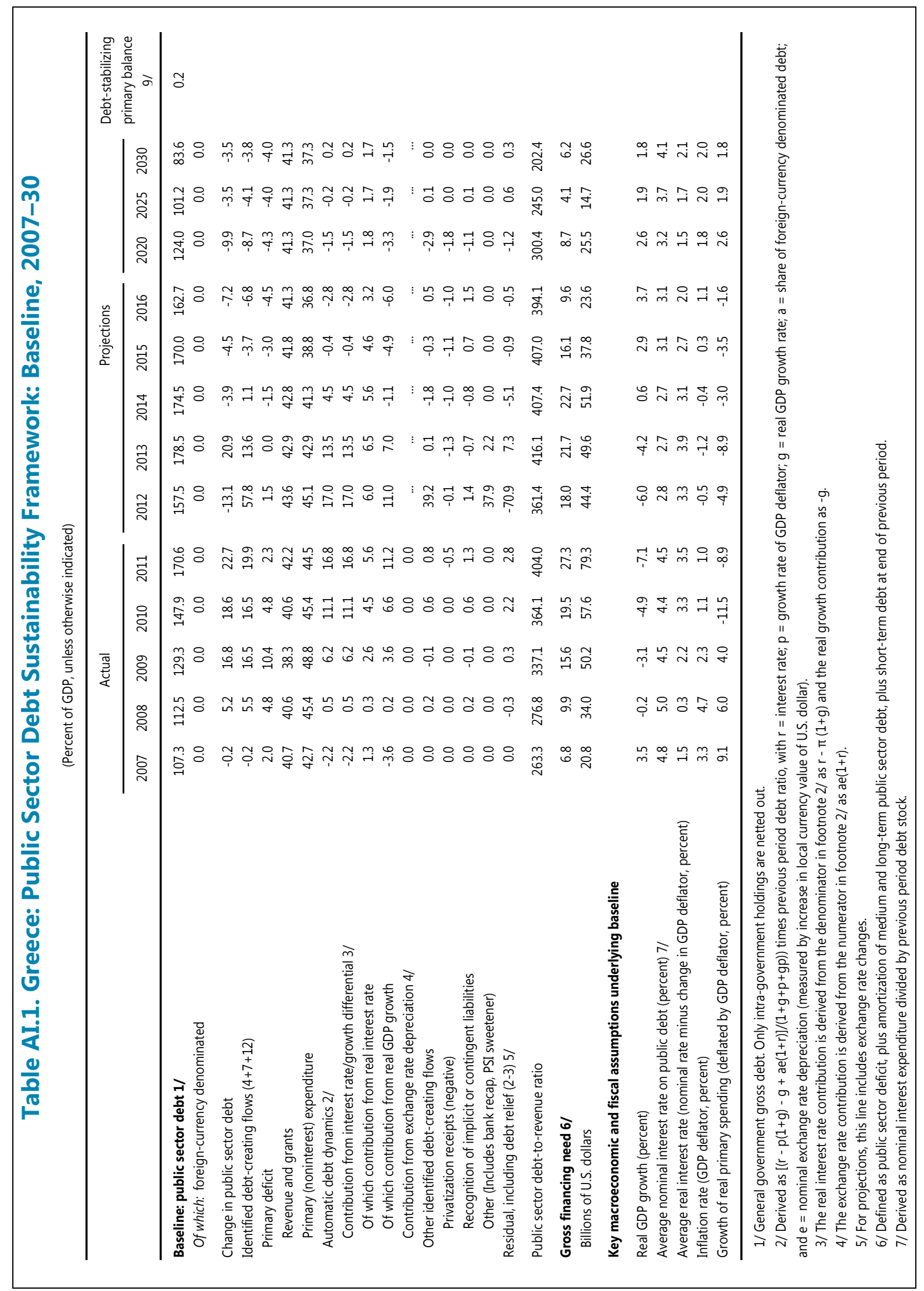




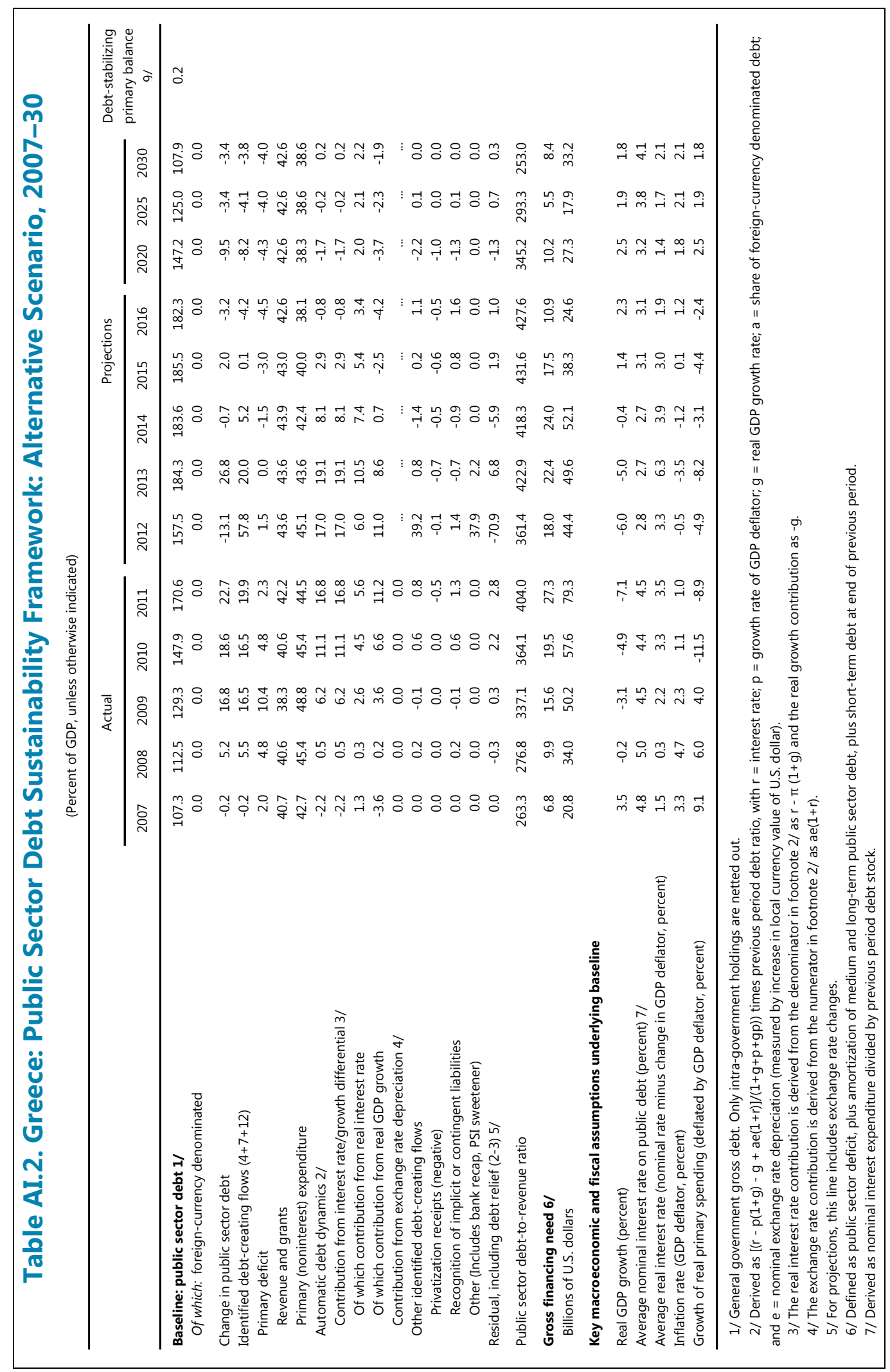




\section{External Sector DSA}

9. External debt would fall significantly by $\mathbf{2 0 2 0}$ according to baseline projections. Gross debt, currently at 235 percent of GDP, would peak at around 260 percent of GDP in 2013 and then drop to 170 percent in 2020. Net debt would fall from 110 percent of GDP in 2012 to 60 percent in 2020. The net-debt path is better than in the original EFF-supported program, despite higher Target 2 liabilities and lower privatization receipts, because the current account has adjusted faster than expected. The projected downward path for debt ratios continues to be consistent with sustainability. Several factors lie behind the projected improvement over time in external debt. Most importantly, the current account is projected to improve quickly as competitiveness is restored and Greece continues to rely on official loans at relatively low interest rates. The agreed reduction in the GLF interest rate and EFSF fees, and the return of SMP profits will also contribute to the sharp improvement in the current account. In fact, the current account will surpass the debt-stabilizing level of -4.6 percent of GDP already in 2012. FDI inflows related to privatization, although lower than in the original program request, remain an important non-debt-creating source of financing.

\section{Macroeconomic shocks and policy slippages could result in adverse dynamics.}

- Larger current account deficits. Slow competitiveness improvements resulting from delayed structural reforms or a terms-of-trade shock would affect exports negatively and worsen the baseline current account projections by around 1.5 percent of GDP. The debt ratio would remain on a downward path but would be 11 percentage points higher than in the baseline by 2020 .

- Interest rate shock. The effects of higher Greek sovereign spreads would be dampened by the almost exclusive reliance of Greece on official financing. Changes in the risk free rate would nevertheless impact Greece through the cost of official financing. A 100bps shock to Bund rates would worsen the income account and result in a 2020 debt ratio 8 percentage points above the baseline.

- $\quad$ Slippages in privatization. The negative shock to privatization considered in the public DSA would also affect external debt dynamics by reducing non-debt-creating flows by $€ 10$ billion. The impact on the debt path would be modest since baseline privatizationrelated inflows are small relative to the overall financial account balance. The debt ratio in 2020 would be around 4 percentage points higher than in the baseline.

11. Debt dynamics would be significantly worse under a combined shock involving lagged program implementation, weak competitiveness, and higher interest rates. The shocks above would likely be individually manageable but would have a more substantial impact in an adverse scenario where they occur simultaneously. The net debt ratio would reach 87 percent of GDP in 2020, 23 percent of GDP higher than in the baseline. 


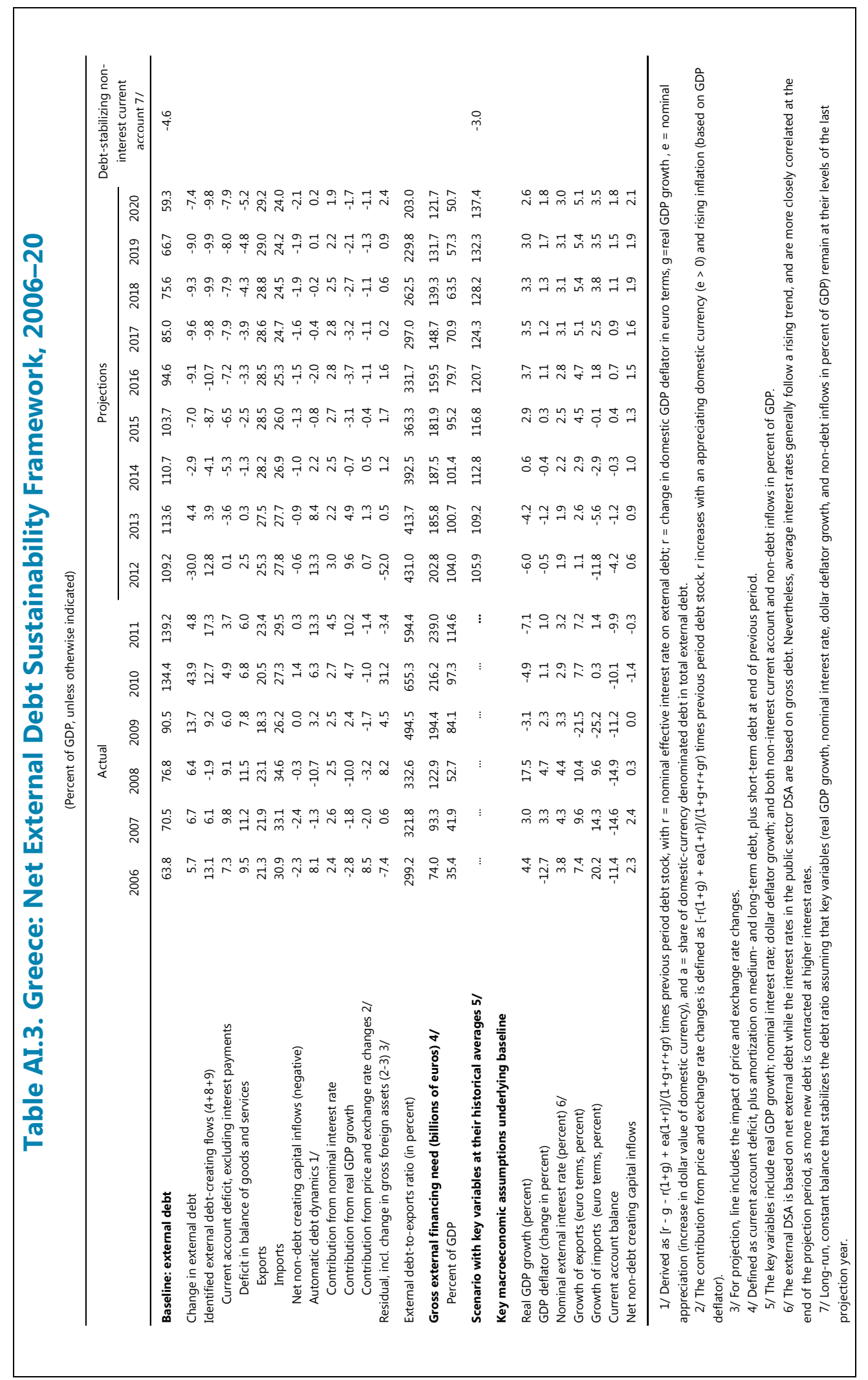


Figure AI.1. Greece: Net External Debt Sustainability: Bound Tests, 2006-20 1/
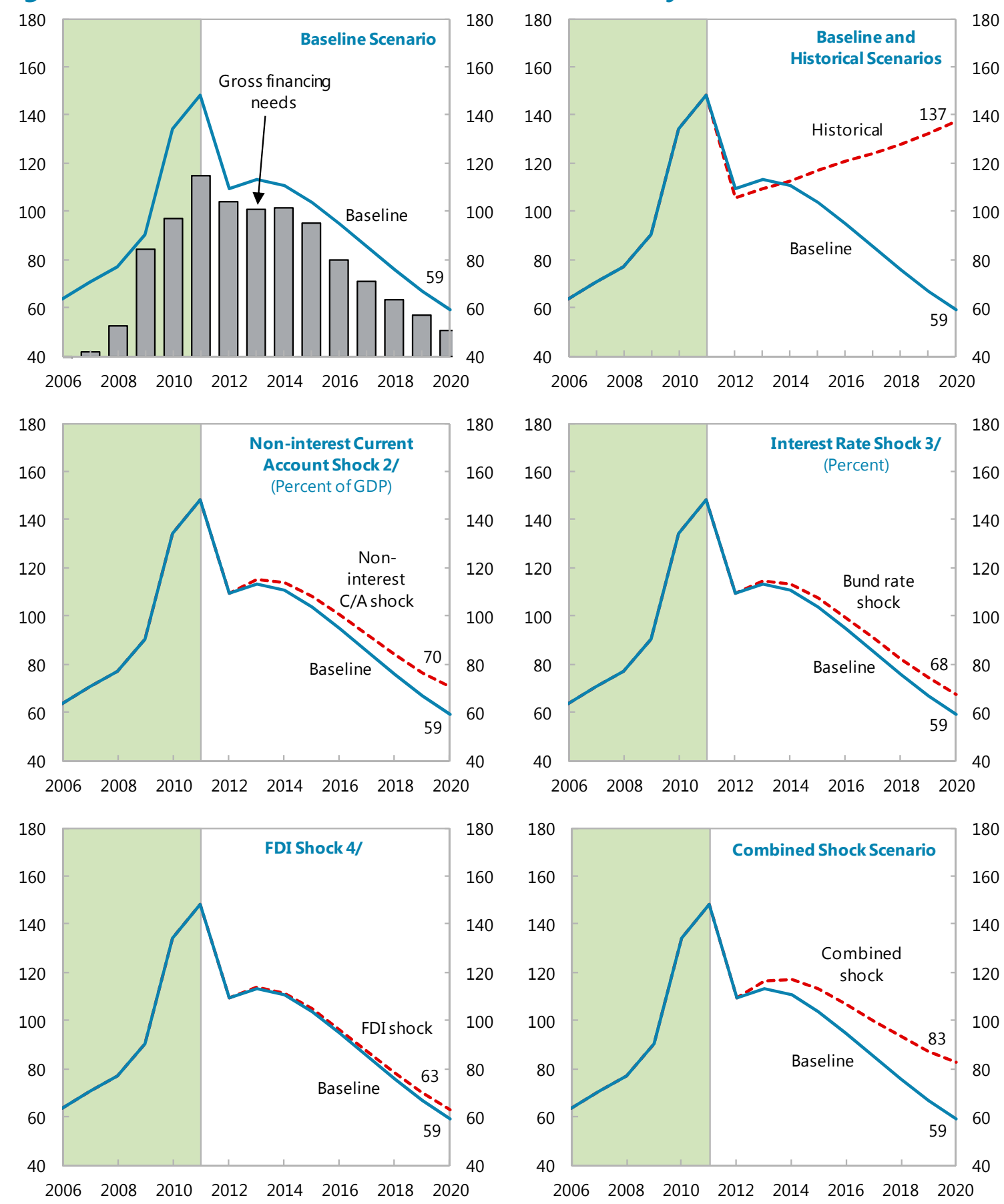

Sources: Greek authorites; and IMF staff estimates.

$1 /$ Shaded areas represent actual/preliminary data. Figures in the boxes represent average projections for the respective variables in the baseline and scenario being presented. Ten-year (pre-crisis) historical average for the variable is also shown.

2/ Current account balance lower by 1.5 percent of GDP due to delayed program implementation and terms-of-trade shock.

3/ Impact of $100 \mathrm{bps}$ shock to Bund rates on Greece's official interestrates and income balance.

4/ Decline in FDI due to reduced privatization receipts. 


\section{APPENDIX II. FUND RELATIONS}

(As of October 31, 2012)

Membership Status: Joined December 27, 1945. It has accepted the obligations of Article VIII, Sections 2, 3, and 4.

Exchange Rate Arrangements: Greece's currency is the euro, which floats freely and independently against other currencies. It maintains an exchange system free of restrictions apart from those resulting from European Council regulations, which were last notified to the Fund in accordance with Decision 144 under EBD/08/35, 4/08.

General Resources Account:

Quota

Fund holdings of currency

Reserve position in Fund

SDR Department:

Net cumulative allocation

Holdings

Outstanding Purchases and Loans:

Stand-by Arrangements

Extended Arrangements

\section{SDR Million}

$1,101.80$

$19,801.97$

240.74

SDR Million

782.36

553.46

SDR Million

$17,541.80$

$1,399.10$
Percent Quota

100.00

$1,797.24$

21.85

Percent Allocation 100.00

70.74

Percent Quota

$1,592.10$

126.98

Latest Financial Arrangements:

$\begin{array}{lcccc}\text { Type } & \begin{array}{c}\text { Date of } \\ \text { Arrangement }\end{array} & \begin{array}{c}\text { Expiration } \\ \text { Date }\end{array} & \begin{array}{c}\text { Amount Approved } \\ \text { (SDR Million) }\end{array} & \begin{array}{c}\text { Amount Drawn } \\ \text { (SDR Million) }\end{array} \\ \text { EFF } & \text { Mar 15, 2012 } & \text { Mar 14, 2016 } & 23,785.30 & 1,399.10 \\ \text { Stand-By } & \text { May 09, 2010 } & \text { Mar 14, 2012 } & 26,432.90 & 17,541.80\end{array}$

Projected Payments to Fund (SDR million; based on existing use of resources and present holdings of SDRs):

\begin{tabular}{lrrrrr} 
& \multicolumn{5}{c}{ Forthcoming } \\
\hline & 2012 & 2013 & 2014 & \multicolumn{1}{c}{2015} & 2016 \\
\hline Principal & & $1,471.81$ & $6,277.79$ & $7,299.092,609.70$ \\
Charges/Interest & 130.00 & 587.80 & 547.77 & 252.15 & 39.04 \\
Total & 130.00 & $2,059.61$ & $6,825.56$ & $7,551.242,648.74$ \\
\hline
\end{tabular}

Implementation of HIPC Initiative: Not Applicable

Safeguards Assessment: An update safeguards assessment of the Bank of Greece (BoG) has been conducted in connection with the current Extended Fund Facility, and was finalized in August 2012. 
The update assessment found that BoG has strengthened its safeguards framework since the 2010 assessment. Financial reporting and external audit practices adhere to international standards and recent amendments to the BoG's Statute are expected to enhance governance and the central bank's autonomy from private shareholders. Emergency lending operations are subject to established legal and control frameworks, but the assessment recommends a compliance review by the BoG's internal audit department.

Last Article IV mission: Discussions for the 2009 Article IV Consultation were held in Athens during May 13-25, 2009. The Staff Report (Country Report No. 09/244) was considered by the Executive Board on July 24, 2009. The report is available at

http://www.imf.org/external/pubs/cat/longres.cfm?sk=23169.0. Article IV Consultations with Greece are currently held on a $24-$ month consultation cycle.

The First and Second Reviews under the Extended Arrangement: Discussions for the $1^{\text {st }}$ and $2^{\text {nd }}$ reviews under the Extended Arrangement were held during July 3-11, July 24-August 5, September 5-20, and October 1-16. The mission met with the Prime Minister, Minister of Finance, Governor of the Bank of Greece, and other Cabinet Ministers; and staff in these and other ministries. The mission also met private banks, think tanks, and employer associations. The staff team comprised P. Thomsen (head), M. Flanagan, W. McGrew, H. Floerkemeier, G. Gottlieb, N. Hobdari, W. Maliszewski, and M. Shamloo (EUR); G. Palomba, and I. Petrova (FAD); S. Lanau (SPR); G. Mitchell Casselle, L. Cortavarria, N. Jassaud, and N. Saker (MCM); and G. Esposito and Y. Liu (LEG). B. Traa, S. Eble, G. Gatopoulos, and M. Athanasopoulou (IMF resident representative office) assisted the mission.

\section{Technical Assistance:}

\begin{tabular}{lll}
\hline Department & Purpose & Date \\
\hline MCM & Banking supervision & March 2010 \\
FAD & Public financial management: initial analysis and priority & April 2010 \\
& reforms & \\
FAD & Revenue administration: initial analysis and reform priorities & April 2010 \\
STA & Data quality and misreporting (K-1 Report) & April 2010 \\
FAD & General tax policy & May 2010 \\
MCM/FAD/LEG & Financial instruments & May 2010 \\
FAD & Expenditure Policy & June 2010 \\
FAD & Public financial management: follow-up on priority reforms & June 2010 \\
LEG & Implementation of financial sector components of the SBA & June 2010 \\
& program & \\
MCM & Implementation of financial sector components of the SBA & June 2010 \\
& program & July 2010 \\
FAD & Tax administration: design of the anti-evasion plan & September 2010 \\
MCM & Implementation of financial sector components of the SBA & \\
& program & September 2010 \\
FAD & Tax administration: implementation of the anti-evasion plan & \\
98 INTERNATIONAL MONETARY FUnD &
\end{tabular}




\begin{tabular}{|c|c|c|}
\hline Department & Purpose & Date \\
\hline FAD & $\begin{array}{l}\text { Public financial management: implementation status of } \\
\text { priority reforms }\end{array}$ & September 2010 \\
\hline STA & Monitoring of fiscal data for the program & September 2010 \\
\hline FAD & Tax administration: anti-evasion and structural reforms & October 2010 \\
\hline FAD & Health system analysis and proposals & October 2010 \\
\hline STA & Government finance statistics & December 2010 \\
\hline FAD & Tax administration: anti-evasion and structural reforms & February 2011 \\
\hline FAD & Role of accounting officers & February 2011 \\
\hline STA/FAD & Government finance statistics/fiscal reporting & March 2011 \\
\hline FAD & Tax administration: strategic planning & March 2011 \\
\hline FAD & $\begin{array}{l}\text { Public financial management: follow-up on implementation } \\
\text { of priority reforms }\end{array}$ & April 2011 \\
\hline FAD/LEG & Tax administration: anti-evasion and structural reforms & April 2011 \\
\hline LEG & Legal framework for privatization & April 2011 \\
\hline MCM/LEG & $\begin{array}{l}\text { Review of the Legal and Operational Framework for Bank } \\
\text { Resolution }\end{array}$ & June 2011 \\
\hline FAD & Tax administration: strategic planning and taxpayer audit & June 2011 \\
\hline FAD & $\begin{array}{l}\text { Tax administration: tax collection and tax administration } \\
\text { reform }\end{array}$ & July 2011 \\
\hline LEG & $\begin{array}{l}\text { AML and anti-tax evasion: strengthening BoG's supervisory } \\
\text { process }\end{array}$ & September 2011 \\
\hline LEG & $\begin{array}{l}\text { AML and anti-tax evasion: review of cross-border financial } \\
\text { flows }\end{array}$ & September 2011 \\
\hline FAD & Safeguarding revenue and encouraging growth & September 2011 \\
\hline FAD & Modernizing the General Accounting office & September 2011 \\
\hline FAD & Preparing the 2012 budgets & September 2011 \\
\hline STA & TA Mission (Fiscal Reporting) & December 2011 \\
\hline FAD & Expenditure Policy: OECD Review of Social Programs & January 2012 \\
\hline FAD & Tax administration & January 2012 \\
\hline LEG & $\begin{array}{l}\text { Reform of central bank governance for banking supervision } \\
\text { and resolution }\end{array}$ & January 2012 \\
\hline LEG & $\begin{array}{l}\text { AML and anti-tax evasion: strengthening BoG's supervisory } \\
\text { process }\end{array}$ & January 2012 \\
\hline FAD & PFM: Accounting Officers and 2013 Budget Preparation & February 2012 \\
\hline FAD & $\begin{array}{l}\text { Tax Administration: Collection and analyzing taxpayer } \\
\text { compliance data }\end{array}$ & February 2012 \\
\hline STA & TA Mission (Fiscal Reporting) & February 2012 \\
\hline FAD & Expenditure Policy: Spending Review Mission & March 2012 \\
\hline FAD & Tax administration: Follow up & March 2012 \\
\hline FAD & Revenue Administration: Social contribution complaisance & March 2012 \\
\hline LEG & AML and anti-tax evasion: strengthening BoG's supervisory & April 2012 \\
\hline
\end{tabular}




\begin{tabular}{lll}
\hline Department & Purpose & Date \\
\hline & process & \\
STA & TA Mission (Fiscal Reporting) & April 2012 \\
STA & TA Mission (Fiscal Reporting) & June 2012 \\
FAD & PFM: Discussions on PFM TA with the new Government of & July 2012 \\
& Greece & \\
FAD & Tax Administration & July 2012 \\
FAD & Revenue Administration: Social contribution compliance & July 2012 \\
FAD/LEG & Tax Policy: Technical consultations on tax policy reforms & September 2012 \\
FAD & Revenue Administration: Social contribution compliance & September 2012 \\
FAD & Tax Administration: IT Audit Techniques & September 2012 \\
FAD & Tax Administration: Tax Audit Case Selection & September 2012 \\
LEG & AML and anti-tax evasion: strengthening BoG's supervisory & September 2012 \\
& process & \\
FAD & PFM: Reform implementation review & October 2012 \\
FAD & Tax Administration: Follow up & October 2012 \\
FAD/LEG & Tax Policy: Technical consultations on tax policy reforms & October 2012 \\
FAD & Tax administration: Organizational Reform & October 2012 \\
FAD & Tax Administration: IT Audit Techniques & October 2012 \\
FAD & Tax administration: Organizational Reform & November 2012 \\
LEG & AML and anti-tax evasion: strengthening BoG's supervisory & November 2012 \\
& process & \\
\hline & & \\
\hline
\end{tabular}

Resident Representatives: Mr. Bob Traa (Senior Resident Representative) and Ms. Stephanie Eble (Deputy Resident Representative) assumed their positions in October 2010. 


\section{APPENDIX III. LETTER OF INTENT}

Athens, December 21, 2012

Ms. Christine Lagarde:

Managing Director

International Monetary Fund

Washington DC

Dear Ms. Lagarde:

In the attached update to the Memorandum of Economic and Financial Policies (MEFP) from March 9, 2012, we confirm our commitment to the policies and objectives of the economic program supported by an arrangement under the Extended Fund Facility (EFF), and describe progress and policy steps towards meeting the objectives under the program.

The needed adjustment of the Greek economy has continued, and the primary deficit has declined considerably. Following far-reaching labor market reforms taken in early 2012 under the program, Greece's competitiveness gap and current account deficit are shrinking perceptibly. However, the economic contraction has been deeper-than-expected in 2012.

We have faced some delays in program implementation. We met most end-September quantitative performance criteria, although we missed by small margins those on domestic arrears and government guarantees (Table 1a). Some progress was made on structural benchmarks, although their full implementation has been delayed (Table 2).

We are bringing the program back on track, and to this end have implemented a comprehensive list of actions (Table 3):

- We are taking far-reaching structural reforms to help lay the basis for economic recovery. As prior actions for the review we have taken a number of steps to help liberalize key product and service markets, restarted the privatization process, and reduced non-wage labor costs. These reforms will strengthen competitiveness, and encourage much-needed investment.

- We are strengthening the banking system, to further underpin the recovery. As prior actions for the review, we have finalized the banking sector recapitalization framework, and introduced reforms to strengthen governance. These actions should strengthen depositor confidence and support renewed bank lending.

- The government has adopted an ambitious fiscal program for 2013-16. As prior actions for the review, we have adopted: (i) a 2013 budget and a revised medium-term fiscal program; and (ii) an implementation bill containing the fiscal measures for 2013-14. Our revised fiscal program targets the $4 \frac{1}{2}$ percent of GDP primary surplus target by 2016 , two years later than 
foreseen in the EFF-supported program. The smoother path will help to moderate the impact of fiscal adjustment on the economy.

- $\quad$ Strengthening fiscal institutions is a crucial part of our reform program. As prior actions for the review, the government has strengthened the capacity of the Tax Revenue Administration, simplified tax accounting procedures, and taken measures to secure tighter control over general government spending. These measures will help improve payment of taxes, and prevent future accumulation of arrears.

We have received new financing commitments from our euro area partners to support our revised policy framework. They have committed to reduce interest rates and fees on existing loans, extend maturities, and have agreed to finance a voluntary debt buyback from the private sector. This will help us to restore a sustainable debt trajectory. More broadly, they have reiterated their commitment to provide adequate support to Greece for the life of the program and beyond, provided that Greece fully complies with the requirements and objectives of the adjustment program.

On the basis of the steps that we have taken to bring the program back on track, we request:

- $\quad$ Completion of the first and second reviews, and a disbursement in the amount of SDR 2,798.2 million. In view of the delays in the program, we request equal re-phasing of the remaining undrawn purchases over the remainder of the arrangement (Appendix I).

- Modification of the end-December 2012 performance criteria on the general government primary cash balance, state budget primary spending, and net change in the stock of domestic arrears (narrow definition).

- Waivers of applicability for the modified end-December performance criteria (second bullet), and also for the performance criterion covering the stock of central government debt and new guarantees granted by the central government. We expect to meet each of these targets.

- Modification of the end-December indicative target on the net change in the stock of general government arrears.

- Establishment of performance criteria and indicative targets for end-March 2013 and endJune 2013; establishment of a quantitative performance criterion on privatization proceeds for end-September 2013; and establishment of indicative targets for end-September 2013, and end-December 2013 (Table 1b).

- $\quad$ Finally, as detailed below, we propose new structural benchmarks to measure progress against our program objectives (Table 4). 
We believe that the policies set forth in the attached MEFP are adequate to achieve the objectives under the program and stand ready to take any measures that may become appropriate for this purpose as circumstances change. We will consult with the Fund on the adoption of any such actions and in advance of revisions to the policies contained in this MEFP, in accordance with the Fund's policies on such consultations.

This letter is being copied to Messrs. Juncker, Rehn and Draghi.

$$
\text { /s/ }
$$

\section{Antonis Samaras}

Prime Minister

/s/

Yannis Stournaras

Minister of Finance

\author{
/s/ \\ George Provopoulos \\ Governor of the Bank of Greece
}




\section{APPENDIX III. MEMORANDUM OF ECONOMIC AND FINANCIAL POLICIES}

\section{Strategy and Outlook}

1. Greece has made progress since the onset of the crisis in 2010 , but continues to face three crucial challenges:

- $\quad$ Restoring growth. Greece is in its fifth year of recession, with unemployment reaching unprecedented levels, particularly among youth. Restoring growth and bringing jobs back requires a deep restructuring of the economy, to shift the engine of growth from consumption to exports and to investment in the tradables sector.

- Securing fiscal sustainability. Despite the adjustment we have undertaken so far, further efforts are needed to restore fiscal sustainability. These will have to spread the adjustment burden fairly across the Greek population. Achieving a sustainable level of debt within the euro area is vital to reduce vulnerabilities and protect Greek citizens from even-deeper cuts that would otherwise prove necessary.

- $\quad$ Securing the financial system. The deep recession combined with the recent public debt restructuring has taken a toll on banks' capital. Recapitalization of the banking system is needed to strengthen depositor confidence and restart bank lending.

2. The short term economic outlook remains difficult, but the adjustment process is now moving faster. The recession has turned out to be deeper than envisaged under the program, while slow progress in structural reforms has not sufficiently addressed price rigidities. However, our external deficit is projected to shrink at a faster pace than expected, and competitiveness gains, measured by unit labor costs, are also expected to come at a faster pace.

3. Against this backdrop, we have adapted policies in the program to better help achieve our goals and in particular to more rapidly stabilize the economy:

- We have made it a priority to implement structural reforms designed to reduce prices and encourage employment. Product market reforms have been frontloaded to increase competition, help reduce prices and thus help protect real household incomes. Privatization will also move forward at an accelerated rate to facilitate new private sector investment. Non-wage labor costs will be reduced, to support hiring and lessen pressure for nominal wage cuts.

- We will make a renewed near-term push to correct Greece's poor business environment. When confidence returns, the government bureaucracy must not stand in the way of new investment and the jobs that this would bring. Accordingly, licensing reforms 
will be fully implemented, export procedures simplified, and the administrative burden of Greece's complex tax system will be simplified.

The government has programmed more time for fiscal adjustment, to help smooth the recession. By taking an extra two years to reach our fiscal targets, we expect the pace of fiscal consolidation to drop from 3 to $1 \frac{1 / 2}{2}$ percent of GDP per year. This will limit the negative growth impacts in 2013-14, when the economy needs to find a firmer footing, while still preserving a good adjustment pace.

Several initiatives will help support demand in the near term. Increased absorption of EU structural funds will help to sustain public investment, while new loans from the EIB to banks will help support new lending to small and medium enterprises (SMEs). The program provides resources to gradually clear government arrears, and we expect this to help improve liquidity in the corporate sector. And to complement our work to recapitalize banks, we will introduce measures to help banks efficiently work out debts with truly distressed borrowers.

- We are enhancing our social safety net to help cushion those who are most deeply affected by Greece's recession. We will leverage available EU structural funds to strengthen job training initiatives, and increase internal resources available for unemployment insurance.

\section{Our European partners have supported our revised policy framework with additional} financing, on terms consistent with restoring Greece's debt sustainability. Our additional financing needs will be covered via a package that provides for lower interest rates, deferred interest and longer maturities on loans, and higher fiscal transfers (the commitment by member states to pass on to Greece an amount equivalent to the income on the SMP portfolio of the National Central Banks as from budget year 2013). They also agreed to finance a voluntary debt buyback from the private sector, which was completed on December 18, 2012.

\section{With our revised policies we expect to be able to stabilize the economy and start a} recovery in the next 12-18 months. Output would still contract by over 6 percent in 2012, and about $4 \frac{1}{4}$ percent next year, before starting to recover, on a quarter-over-quarter basis, toward end2013 or early-2014. Moderate inflation in 2012 would give way to mild deflation in 2013. The current account would be broadly in balance by 2014. But to meet or, hopefully, exceed these projections, we recognize the critical importance of strong and timely program implementation, to support a return of confidence.

6. Greece has a positive outlook over the medium term. Growth is projected to accelerate once the envisaged fiscal adjustment advances and structural reforms translate into improved competitiveness. Over the medium term, inflation in Greece is expected to remain below levels in our trading partners and our competitiveness gap should close. This should help deliver a current account surplus around 2017. Against this macro backdrop, and given our financing arrangements, 
we expect public debt to fall to 124 percent of GDP by 2020, and to substantially below 110 percent of GDP by 2022. We also expect the reliance of our banking system on the Eurosystem to gradually decline.

\section{Structural Reforms}

\section{The government is determined to reinvigorate structural reforms to strengthen the} basis for economic growth. In particular, we are committed to comprehensively liberalize product and service markets by removing unnecessary restrictions and barriers to entry that currently impede competition and downward price adjustment. We also continue to recognize the importance of ambitious business environment reforms to remove barriers to investment. In addition, with the unemployment rate unacceptably high, we are determined to implement fully recent labor market reforms and to take further steps to improve the functioning of the labor market and facilitate employment creation.

\section{As a matter of priority, we will accelerate liberalization of product and service markets.} Citizens have made significant sacrifices by accepting lower wages and benefits. Productions costs in the economy have declined, but output prices have not dropped commensurately. To deliver more efficiency and productivity across the economy, and higher competitiveness, we have adopted a package of liberalization measures as a prior action for the review (Annexes I.1-I.2):

- Retail market. We have repealed unnecessary restrictions, deriving mainly from sanitary, labor, and transport regulations. These changes will allow a wider class of goods to be sold by retailers, and reduce retailers' operating costs. This in turn should help contribute to lower prices and more choice for consumers.

Fuel market. To allow downward adjustment of prices, legal changes have, inter alia, allow small fuel retailers to import fuel without needing to construct large fuel storage facilities; and permitted independent retailers to buy directly from refineries and to transport their own fuel. Taxes on diesel and heating oil have been equalized. We will guard against smuggling and fraud by introducing new control mechanisms in fuel distribution and retailing.

Transportation services. To reduce transportation prices and strengthen the competitiveness of our tourism sector, we have: (i) removed restrictions on the rental of pickup trucks and vans, chauffeur services; and (ii) allowed shuttle services by hotels and tour agencies using small vehicles (less than 12 seats) and tour packages for small vans and off-terrain vehicles.

Regulated professions (Annex I.2). Our review of earlier efforts has revealed areas where further and more ambitious efforts are needed. We have focused on eliminating inconsistencies between specific legislation and the 2011 law (3919/2011) on professions, minimum fees for services, and mandatory use of services in selected professions. We have also tackled a new group of 8 professions and activities of economic importance (including 
custom brokers, stevedores in ports, tourist agencies, and private education establishments). As a general principle, the government is committed not to extend new reserved activities to specific professions.

\section{We have also defined several next steps:}

$>$ We remain committed to liberalizing all regulated professions by end-2012 per the 2011 law (3919/2011) on professions (structural benchmark). To confirm our progress in this area, by May 2013 we will complete a study of the 20 largest professions examining the degree to which they have been liberalized, including results with respect to new entrants and price changes.

$>$ We will complete a new round of liberalization of transportation services by endFebruary 2013, with the aim to strengthen the competitiveness of our tourism sector. We will address restrictions affecting airports, seaports, domestic ferry services (in particular covering labor arrangements and routing), and inter-urban travel. These processes have been launched in October 2012.

$>$ Finally, prices of a number of key goods and services are high in Greece compared to other EU countries, particularly those for construction materials (including cement and steel). Accordingly, using the OECD toolkit and with the help of the Hellenic Competition Commission and other regulators, the government will screen the restrictions in these sectors, and also in the important food processing and tourism sectors. We will prepare by end-June 2013 an action plan to promote competition and facilitate price flexibility in these sectors.

\section{The government will also accelerate improvements in Greece's business environment.}

While Greece has passed much legislation in this area, our overall ranking in Doing Business Indicators remains unacceptably low. Our priorities include:

- Licensing and regulation. The government will focus in the following three areas. First, by end-December 2012 we will further simplify procedures for establishing companies, including by allowing the use of model company statutes, streamlining background checks on company founders, and reducing the minimum capital requirements of new companies in line with best EU practices. Second, we will publish during Q4 2012 a national trade facilitation strategy with time-bound steps to simplify pre-customs and customs procedures and to increase working shifts (Athens airport and Piraeus port will shift to $24 / 7$ by endMarch 2013). Third, by Q1 2013 we will complete the legal framework for the implementation of licensing laws, especially on manufacturing activities and environmental projects and activities. For the latter we will certify bodies to issue establishment and operating licenses and to lay down the procedures and criteria for licensing.

- Judicial reform. We will continue with measures to reduce case backlog and improve the 
efficiency of the judicial system. We will develop by end-January 2013 an action plan with specific measures to reduce the non-tax case backlog. Every quarter, starting from endDecember 2012, we will publish a report on progress in backlog reduction, and update the work plan to reduce the tax case backlog (with priority on cases exceeding $€ 1$ million). Separately, to inform our efforts to streamline the judicial process, a paper outlining the main proposals for amendments to the Code of Civil Procedure will be prepared by endMarch 2013.

- Next steps. With assistance from the OECD we will screen legislation in a number of areas (including the agriculture, energy, fisheries, and pharmaceutical sectors, as well as public procurement) with a view to reduce the administrative burden. On the basis of the findings of these reviews, we will propose by end-September 2013 the needed amendments to our legal framework. We will also, by end-September 2013, produce a comprehensive list of nuisance taxes and levies, and approve a plan to incorporate them into the central government budget (along with the associated spending), and to eliminate the majority of them in a budget neutral way in the 2014 budget (structural benchmark).

\section{The government intends to build on recent labor market reforms to further improve} the functioning of the labor market and encourage job creation: The labor reforms adopted in February 2012 under the program have already helped to lower unit labor costs. The government will sustain the reforms and apply them uniformly across the sectors to which they apply. However, the minimum wage system in Greece remains complex and delinked from broader labor market conditions, while non-wage labor costs remain excessive, contributing to pressure for excess nominal wage declines and placing barriers in front of employment creation. We have specified measures to tackle these issues, and the next steps towards a stronger labor framework for Greece:

As a prior action for the review (Annex II), the government has established a timetable to reform Greece's minimum wage. Under the new framework, the government will put in place a minimum wage mechanism adopted by parliament following a proposal by the government (to be made after consultation with social partners and other stakeholders and independent experts). The system will become effective by end-March 2013. At this point, the statutory minimum wage and maturity allowances will be set equal to the levels agreed at program approval in March 2012 and frozen at these levels. There will be no other allowances. Collective agreements will remain free to set higher wages than the statutory level set by the government, and free to set allowances of various types, but these will be binding only for the signatories of the agreements. By Q1 2014 the government will assess whether the mechanism is sufficient to address unemployment and improve competitiveness, and whether it is simple enough to be effectively administered by companies. 
To reduce nonwage labor costs, we will take a number of actions:

> Tax wedge. By November 30, 2013, as a new program structural benchmark, we will adopt legislation reforming the system of social security contributions to: (i) broaden the base for contributions; (ii) simplify the contribution schedule across the various funds; (iii) shift funding away from nuisance taxes and onto contributions; and (iv) reduce contribution rates by an average of 5 percentage points (from the rates prevailing on September 30, 2012). The reforms will be phased in on January 1, in 2014, 2015, and 2016 and will be revenue neutral and preserve the actuarial balance of the various funds. As an intermediate step, by end-September 2013 we will complete actuarial studies of possible changes and propose an action plan.

$>$ Other non-wage labor costs. As a prior action for the review, the government has adopted legislation to reduce other nonwage labor costs. The changes will bring Greece closer to EU partners, and include (Annex II): (i) greater efficiency of labor arrangements within the overall 40 hour weekly limit on working time (covering the maximum number of workdays, working hours, and shift and leave restrictions); (ii) a reduction in administrative burden (related to extensive reporting and preapproval requirements of work arrangements); and (iii) lower dismissal costs (with grandfathering for existing workers, subject to a cap).

- We will work to strengthen the safety net for those who become unemployed. As discussed below in the fiscal policy section, the government is enhancing unemployment benefits to help mitigate any short-term impact that labor market reforms may have. We will also work to improve the effectiveness of our training and job-matching programs, focusing on the young and the long-term unemployed (and better leverage any available structural funds to this end).

Next steps. As a general principle, we are committed to address any remaining features of our labor market that fall short of best European practices. As a first step, an independent assessment of the labor inspectorate will be completed by end-2012, with a particular focus on effective and efficient control procedures to fight undeclared work. The government will use this as an input to help specify an action plan to implement procedures to more effectively detect cases of undeclared work, and to eliminate activities that increase administrative costs for firms without serving a justifiable public policy objective.

\section{In light of the deep problems we have had to date with fully implementing structural} reforms, the government will strengthen its management and monitoring mechanisms in this area. We will fully staff the directorate of planning, management, and monitoring of reforms at the Office of the Prime Minister and publish on a quarterly basis monitoring indicators for each reform initiative on the government's website. 


\section{Privatization}

12. It is a priority of the government to restart and invigorate Greece's privatization program. Greece has been too slow to date in achieving the benefits this can bring. Shifting government assets to the private sector should improve efficiency and reduce prices and catalyze needed investment. It will also help to cover budget financing needs, reduce public debt, and improve market sentiment. The program in this area covers the identification of assets, their transfer to the Privatization Fund, measures to overcome obstacles to sales, and measures to strengthen the institutions executing the work.

13. We have made progress in specifying assets for privatization (Appendix II). The list includes state enterprises and concessions in gaming, utilities, and infrastructure, and will also contain bank assets either in possession of the government or to be acquired during the recapitalization process. These assets have an estimated value of $€ 26$ billion. Further, we have screened 81,000 real estate properties with an estimated value of $€ 20-28$ billion. An updated privatization plan has been presented to parliament in the context of the 2012-16 MTFS.

14. The government has taken steps to restart the sales process. Implementation of selected steps defined in the bullets below will be a prior action for the review (see Annex III for details):

- Transfer of assets. The Privatization Fund (HRADF) has been given full and direct ownership of the non-real estate assets included in Annex III. It has also been given full and direct ownership of the real estate assets Kassiopi, and Afantou, the 28 government buildings that will be sold and leased back, and Astir Vouliagmenis. All line ministries and other relevant entities will provide their property registry to the General Secretariat for Public Property.

- Legal framework. To enable transactions to proceed without further delays, we have: (i) removed restrictions to private ownership and control of firms; and (ii) initiated the process to obtain the zoning and land planning permits (ESCHADA) for two real estate projects (Afantou and Kassiopi). As a general principle, upon privatization of each object, we will amend all statutory provisions (including on labor relations) to fully align them with private sector law.

- Advisors. We have appointed all the necessary advisors to prepare the assets that are in the privatization plan for 2012-13.

- Tender process. Government stakes in OPAP, the state lottery, and IBC have been put up for tender.

15. We expect significant further progress in the period ahead: We will establish regulatory frameworks for water companies (December 2012), airports (January 2013), the State Lottery and for ports (April 2013). Furthermore, Egnatia Motorways will be put to tender by March 2013; and we will fully identify and describe 40 new real estate assets that comprise the privatization projects for real estate lots 2 and 3. These 40 real estate assets will be transferred to the HRADF by March 2013. We 
will fully identify and describe all the remaining assets in the pool of 3150 assets that have been preselected and pre-valued by the HRADF. We will transfer full and direct ownership of all commercially viable assets amongst these to the HRADF by end-2013, targeting 250 transfers per quarter beginning in Q1 2013. There will be no transfer or withholding of any real estate assets to entities other than the HRADF, including municipalities and the recently established pension fund SPV, or other dedicated legal entities, without prior consultation and agreement with the HRADF and the $\mathrm{EC} / \mathrm{ECB} / \mathrm{IMF}$, or until such time as the assets necessary to supply the privatization plan have been secured.

\section{The government is committed to insulate the privatization process from political} pressures. The government will take appropriate steps, including changes in existing legislation and/or in the composition of the Board, to safeguard and strengthen the independence and the functioning of the HRADF, if targets for the sale of assets are missed substantially for two consecutive quarters. To ensure that the HRADF is accountable in its role we will enhance the transparency of its operations. To this end the HRADF will publish: (i) a semi-annual update of the Asset Development Plan, which will include a Portfolio Overview, with a description of the assets it manages for privatization a timeline of planned tenders and targeted total receipts for the current and next year; and (ii) quarterly reports on its steps to facilitate privatizations, and financial accounts (including a profit and loss statement, a cash flow statement, and a balance sheet), no later than 60 days after the conclusion of every calendar quarter.

\section{We have adjusted our targets for privatization proceeds to reflect recent delays and} the general deterioration of Greek asset prices. We expect it to take more time (beyond 2020) to realize the full amount of proceeds of $€ 50$ billion, but we will proceed as quickly as possible. We expect, cumulatively (from June 2011), at least $€ 5.9$ billion through 2014, $€ 10.5$ billion through 2016, and $€ 25.6$ billion through 2020. In line with these cash targets, we will refrain from selling assets in exchange for government bonds. We will continue to monitor progress via quarterly indicative revenue targets, and via a performance criterion for end-September 2013. In the sixth review under the program, we will take stock of our progress through a special focus on privatization.

\section{Financial Sector Policies}

\section{It is an overarching objective of the government to ensure a viable and well-} capitalized banking sector. This is a necessity to underpin Greece's medium-term recovery. Our strategy is to recapitalize banks while maintaining protection of depositors as a policy objective, minimizing fiscal costs, and keeping the banks under private ownership and management to the extent possible. Our strategy comprises four key pillars: (i) recapitalization of viable banks; (ii) orderly resolution of nonviable banks; (iii) restructuring of banks; and (iv) strengthening governance, supervision and regulation. 


\section{We have now finalized our framework for recapitalizing banks:}

- Strategic assessment of the banking sector. The BoG completed this in March 2012, assisted by an international consultancy firm. The study assessed the viability of the banks based on quantitative and qualitative criteria. This study identified four core banks accounting for about 75 percent of banking sector assets.

- Capital needs. By end 2012, we will align our capital metrics to the minimum core tier I ratio of 9 percent of risk-weighted assets set out in the EBA recommendation on capital buffers. Banks will also have to meet the requirements we have set under Pillar II (to maintain a 7 percent core tier 1 capital ratio under a three-year adverse stress scenario). To this end, the BoG has, as a prior action, informed all banks of their individual capital needs and has requested that they finalize the capital raising process by end April 2013. These capital needs account for the impact of PSI, the valuation losses on new Greek government bonds, and results of a stress test exercise with a three-year horizon (which took into account BlackRock credit loss projections and banks' future pre-provisioning results). The BoG will publish a detailed report on individual banks' capital needs, the recapitalization process, and the methodology followed by end December 2012.

- Capitalization process. The legal framework for the recapitalization has been put into place (prior action). The recapitalization process will involve three broad steps:

> First, the HFSF will provide sufficient funds in the form of bridge capital to bring the core banks up to the minimum level of 9 percent CT1 under Pillar 1 before end-2012. The HFSF will also issue a commitment letter to subscribe to 100 percent of the remaining capital needs.

$>$ In the second step, by end January 2013, the HFSF will subscribe to 100 percent of any convertible instruments that the banks will decide to issue.

$>$ In the third stage, by end April 2013, the core banks will complete the rights issue and any shares not subscribed by the private sector will be acquired by the HFSF subscription to the common equity; all four core banks will meet the capital requirements set by the Bank of Greece (structural benchmark).

- Current or new shareholders will have control of the core banks, provided they are deemed fit and proper as already envisaged in the regulatory framework, and have subscribed no less than 10 percent of the capital to be raised by way of common shares. While existing shareholders will be diluted during the recapitalization process, they or new investors will be allowed to participate in the rights issuance and, should the above 10 percent threshold of private sector participation be reached, will receive warrants to acquire the remaining shares from the HFSF within five years. 
- Subsidiaries have been recapitalized by their parent banks. Agreements have been reached on the acquisition of Emporiki and Geniki by Alpha Bank and Piraeus Bank, respectively, with a view to achieve further consolidation of the banking system while protecting the public sector from potential losses. We expect to approve these acquisitions subsequent to the completion of the due diligence process. These acquisitions will not require injection of additional public funds. been finalized. Our strategic assessment shows that these institutions are less suitable candidates for public money. These banks must be fully capitalized by end-April 2013. They may also merge with other banks if they can demonstrate a credible business plan, meet recapitalization needs by April 2013 and address all viability challenges.

20. We will complete the resolution of undercapitalized banks by June 2013 and establish a framework to manage the assets of banks under liquidation:

- State-owned banks. ATE bank was resolved in July 2012 through a promptly conducted Purchase and Assumption (P\&A) transaction with Piraeus Bank. The final resolution cost will be defined by an external audit of the transferred assets and liabilities. Additionally, Piraeus Bank has presented to the EC a restructuring plan to demonstrate that the new integrated bank will enhance its viability and cost-efficiency. Regarding Hellenic Postal Bank, we have initiated its orderly resolution with the aim to do so via a P\&A transaction to be completed no later than end-January 2013 (structural benchmark). Finally, we will complete Nea Proton's restructuring by mid-May 2013 in an open bid process under the sponsorship of the HFSF.

- Other undercapitalized noncore banks. If the shareholders or new investors are unable to support these institutions as required above by end-April 2013 we plan to complete the resolution of these institutions no later than end June 2013 via P\&A transactions with well capitalized banks, or, as a second best, the establishment of a bridge bank (structural benchmark). To prevent market distortions and unsound banking activities, the BoG has placed all undercapitalized non-core banks under enhanced supervision.

- We will ensure that the assets of banks under liquidation are managed on the basis of best international practices. This includes the bad assets of banks that are resolved as part of the restructuring process. To this end, by end-February 2013 the BoG will publish an assessment report prepared by an international expert regarding policies and procedures required to ensure effective bank asset management and recovery. The report will identify the areas that could require further strengthening to maximize loan collection and help reduce bank resolution costs.

21. We are developing a strategy to address the on-going challenges for the cooperative banks. By end-February 2013, the BoG will complete its assessment of this sector and issue a final 
report. Based on this report, by end-May 2013, we will set out a comprehensive strategy to implement its recommendations.

\section{We have reviewed our estimate of resources needed to fully recapitalize the Greek}

banking system. Based on the strategy laid out above, we still estimate that the funds required will amount to $€ 50$ billion, which is fully accounted for in updated estimates of program financing. We will take no fiscal policy actions to increase this burden (and in particular, no dividends will accrue or be paid to the government on preference shares until the banks are profitable). A one-time $€ 550$ million fee, to be received from banks in 2012 in return for the provision of bridge capital, will be earmarked to the HFSF and placed in the HFSF intermediate account.

\section{The government of Greece has established a framework to ensure continued restructuring and strengthening of the banking system after the recapitalization process is complete:}

- Banks that receive state aid will need to provide clear and realistic business plans for their restructuring:

$>$ Operational restructuring. Following the recapitalization of the core and noncore banks, all institutions should update their restructuring plans and submit them for validation by the EC. These should be finalized by end-June 2013; however, banks that acquire other institutions through P\&A transactions sponsored by the HFSF will have until end-July 2013 to submit such revised plans (structural benchmark). The restructuring plans should take into consideration the updated macroeconomic framework set out in the most recent program review. The HFSF will monitor banks' adherence to their restructuring plans and report on a semi-annual basis to the EC/ECB on progress.

> NPL resolution. The HFSF will request that banks assess, by June 2013, whether their established frameworks and policies to deal with troubled assets are effective. International work-out specialists should be invited to assist in the process.

Funding. As part of the new restructuring plans, banks will set out their intentions to broaden their funding base and reduce over time their reliance on emergency liquidity provided by the central bank. The BoG, following the procedures and rules of the Eurosystem, will stand ready to continue disbursing adequate and appropriate emergency liquidity support in a timely manner if needed.

- We will strengthen the insolvency framework. In consultation with the EC/ECB/IMF staff, we will by, end-January 2013, on the basis of best international practices, review the insolvency framework for households and SMEs as well as the framework for out of court negotiations between banks and troubled borrowers and prepare an assessment identifying areas for improvement. The aim will be to achieve predictable, equitable and transparent 
allocation of risks among all interested parties and maximizing value for the economy in general. By end-February 2013, we will revise, with technical support of international experts, the existing framework to facilitate workouts with over- indebted household borrowers that preserves bank solvency and credit discipline, avoids the use of fiscal resources to protect private borrowers and minimizes moral hazard by targeting borrowers that are in real need. We will refrain from supporting any initiative that may undermine the payment culture in Greece.

- Follow up stress testing. To ensure that the system remains sufficiently well-capitalized, the BoG will, by end-December 2013, conduct a new stress test exercise, based on end-June 2013 data, using a methodology determined in consultation with the EC/ECB/IMF staff (we propose to shift the existing structural benchmark on this action to this new date).

\section{We are committed to ensure that the financial system operates with maximum safeguards to ensure stability and continued viability:}

The HFSF will complete the due diligence of core banks. This will, inter alia, focus on a review of governance including loans to related parties, asset quality, and risk concentration, and any findings of interest to the supervisor have been communicated to the BoG (prior action). The BoG will address these promptly, including via removal of board members and managers, and/or suspension of private shareholders (which would prevent them from participating in bank recapitalization framework). By end-March 2013 banks will submit to the BoG plans to address identified operational governance weaknesses with clear timetables for full implementation by end-December 2013.

- Monitoring trustees will be appointed in all banks under restructuring to submit quarterly reports on governance and operations, as well as ad-hoc reports as needed. The monitoring trustee will work under the direction of the $E C$, within the terms of reference agreed with the EC/ECB/IMF staff and will liaise closely with the EC/ECB observers at the HFSF and share their report with the HFSF. In line with the EU state aid rules the trustees will be responsible for overseeing the implementation of restructuring plans. This includes, inter alia, verifying proper governance and the use of commercial-basis criteria in key policy decisions even in the absence of an approved restructuring plan. The monitoring trustees will closely follow the banks' operations and shall have permanent access to Board meeting minutes, and be observers at the executive committees and other critical committees, including risk management and internal audit functions. These trustees shall be a respected international auditing or consulting firm (that will include the participation of overseas based partners and managers) which needs to be endorsed by the EC on the basis of its competence, its independence from the banks and the absence of any potential conflict of interest. We will agree a terms of reference with the EC/ECB/IMF staff and communicate this to the banks with instructions for the trustees to begin work no later than mid-January 2013 (prior action). 
- We will ensure adequate reporting of HFSF operations, and enhance the EC and ECB observers' access to information at the HFSF. Starting at end-January 2013, the HFSF will initiate semi-annual public reporting on its main activities. As a prior action, we have amended HFSF by-laws to clearly stipulate that the HFSF Board, including the observers, must be informed of all decisions of the core banks having an impact on the HFSF's rights as a shareholder/investor. This information, as soon as received by and through the senior executive of the HFSF, is to be provided a minimum one day in advance of any meeting by the HFSF Board that discusses the bank's decisions.

- We will ensure arms length governance of core banks' business activities. By end-March 2013, the HFSF will publish relationship frameworks with each bank on the basis of best international practices, with a view to define the responsibilities of bank managers and board members and the role of HFSF as a shareholder, to ensure the core banks are run on a commercial basis. A draft for discussion based on international best practices will be developed with the EC, ECB, and IMF staff by end-January 2013.

\section{With a view of adapting the supervision of the Greek banking sector to the changed banking environment we are taking important steps:}

- Updating the supervisory model. The BoG will complete a review of its supervisory approach in light of the new challenges ahead, by end-June 2013, with technical support provided by a banking supervision expert. Key enhancements will include: (i) the refocusing of our off-site analytical capacity to assess the business models of the core banks and be able to monitor and critically analyze the implementation of their business plans and the direction that banks may be taking; (ii) updating onsite supervisory procedures and prudential regulation in light of the findings of the ongoing reviews; and (iii) an action plan to monitor credit risk concentration, in line with best practices, such as enhancing the monitoring of large business groups (including those related to bank owners) by end-July 2013.

Standardizing asset quality disclosure. To enhance information available on banks' asset quality, provisioning and income recognition, the BoG and Hellenic Capital Markets Commission will issue guidelines in accordance with their respective competencies, in order to align banks' disclosure practices to international best practices. This is particularly relevant regarding the accounting for provisioning and reporting of restructured loans. By endAugust 2013 with the assistance of a leading consulting firm, such practices will be benchmarked against those followed by top European institutions and will be reflected in banks' end-2013 financial statements.

- Undertaking a Basel Core Principles (BCP) assessment. We will request the IMF to undertake a stand-alone assessment by end-2014. The BoG will prepare in advance (by endJune 2014) a self-assessment of compliance with the BCP with the support of independent experts. 
- Clarifying competencies and responsibilities. We will develop and publish a memorandum of understanding governing the relationship of the HFSF as a shareholder and the BoG's role regarding the oversight of banks that have received state aid.

\section{Fiscal Policy}

26. The government is determined to bring the fiscal deficit to a sustainable position in a socially balanced and fair manner. We remain committed to achieve a general government primary surplus of $4 \frac{1}{2}$ percent of GDP in the medium term, and to implement the EU fiscal compact thereafter. However, the deeper-than-expected recession calls for reconsidering the pace of fiscal consolidation, and by extension the phasing of fiscal measures. As anticipated in the EFF, we have specified our adjustment strategy.

\section{Our fiscal adjustment path has been extended to help soften the impact of the} recession. Our revised targets for the general government primary balance involve a deficit of $1 \frac{1}{2}$ percent of GDP in 2012, and an evenly-paced improvement in the primary balance thereafterby $1 \frac{1}{2}$ percent of GDP each year to 2016 . In nominal terms, we expect the overall balance to improve from a deficit of $€ 13.8$ billion in 2012 , to a deficit of $€ 8.9$ billion in 2013 , to an endpoint deficit of $€ 1.3$ billion in 2016. The pace of fiscal adjustment would be sped up to the extent that our privatization targets are missed and we have less available financing (by half of the privatization shortfall, up to $€ 1$ billion in additional adjustment per year).

28. The new adjustment path, and different macroeconomic background, has implications for the amount and phasing of needed measures. Needed measures now amount to $€ 13 \frac{1}{2} 2$ billion during 2013-14, comprising $€ 3$ billion to replace previously agreed measures where yields are declining, and $€ 101 / 2$ billion in net new measures. Taking into account a cyclical rebound, and depending on our success with improving government efficiency, we may need $€ 2-4$ billion in measures to close the gap during 2015-16 (see paragraph 32). The figure for 2013-14 is larger than what we expected at the time of program approval, reflecting the longer and deeper recession, as well as delays in implementing earlier measures, and health spending pressures. Without the extension of the fiscal adjustment period, the measures needed for 2013-14 would have amounted to an estimated $€ 20$ billion (including $€ 13$ billion in 2013).

\section{Our adjustment strategy targets known structural problems in the operations of the general government:}

- First, and foremost, we need to improve revenue collections to ensure the adjustment burden is shared fairly across the Greek population. Thus the government has adopted a farreaching tax administration reform strategy, which we are already implementing, and which is explained in detail in the next section. We will aim for revenue gains from this source of $1 \frac{1}{2}$ percent of GDP, the full effects of which are expected to materialize gradually through 2016. 
- $\quad$ Second, the strategy focuses on improving government efficiency. We have already reduced central government operating expenditures over the last two years, but there remains some scope to cut further and to reduce waste, particularly in the health sector. There also remain inefficiencies in the outer reaches of the general government-the extrabudgetary funds, local governments and state enterprises that have not fully caught up with the adjustment effort. This process of rooting out inefficiency will involve adjustments to the high level of government employment.

- Third, the strategy aims at reductions in spending items that grew disproportionately prior to the crisis. Despite measures taken in 2010, the share of pensions in GDP remained high by European standards. Therefore, we needed to reduce it to a level that Greece can afford to finance internally, but we needed to avoid cuts that would bear disproportionately on the most vulnerable. Further, most social transfers are not targeted to the poorest segments of the society. As now legislated, we will refocus them and increase their effectiveness in alleviating poverty.

- Lastly, we intend to establish a more efficient tax system. We need to broaden the base by closing loopholes and adjusting allowances in a way that raises revenue while making the tax system more fair and equitable. We expect this effort to also help our tax collection efforts, by preventing under-reporting.

30. Building on this strategy, our adjustment package through 2014 focuses mainly on permanent spending reductions. In 2013, we need to take $€ 9.2$ billion of measures, and we recognize that the reduction of the wage bill, pensions and social transfers contained in this package (€6 billion) is essential to its credibility. Passage of the 2013 budget and updated MTFS will be a prior action for the review, and adoption of package of fiscal measures, drawn from the areas listed in the following bullets, will as also be a prior action for the review (see Annex $V$ for a detailed description of the measures):

- Public sector wage bill. We aim to reduce the wage bill, net of taxes and social contributions, by $€ 1.5$ billion from 2012 to 2014:

$>$ Public sector employee compensation. We have adjusted the wage grid for special regimes, with effect from August 1, 2012. We have reduced labor costs associated with elected positions, and eliminate seasonal bonuses and suspend the payment of performance bonuses in the public sector. Finally, we will also adopt legislation to align the wage grid of parliamentary staff with the new wage grid for state employees.

$>$ Public administration reform. To achieve a leaner more efficient state, we have initiated a rigorous evaluation of administrative structures and personnel. We will combine this assessment with mobility, attrition, reduction of temporary contracts, disciplinary procedures, and mandatory redundancies. This should help reduce the public sector workforce by 150,000 by 2015 , relative to the end-2010 level, while 
allowing room to hire staff with critical skills to support specialized government operations. The key building blocks for this include:

- A rigorous assessment. The Ministry of Administrative Reform will complete a functional review for government ministries, by January 2013, and individual staff performance assessments by end-2013. This reform process will be extended to extra-budgetary funds and regional and local administrations in 2013.This will identify entities to be merged or closed, staff that are redundant, staff that are not fully qualified for their positions, and open positions where new staff are needed.

- Mobility and exits. Entity closures and cancelation of redundant positions will be pursued, and all affected employees will be either transferred to a mobility scheme or dismissed. Concerning those transferred to a mobility scheme, affected employees will be provided with one year of reduced pay. Our target is to place 27,000 in this scheme by end-2013. If they fail to find a new position in the public sector, they will be required to transition to the private sector. To facilitate renewal of the public sector workforce we expect a large share of those entering this program to ultimately transition to the private sector notably via mandatory exits. We expect to complete staffing plans for line ministries by end-February 2013, and will use these plans to identify redundant positions and employees, and on this basis set quarterly targets for mandatory exits through end-2014 (proposed as a new structural benchmark).

- Hiring controls and plans. To ensure that our efforts to redeploy and reduce personnel are effective, we will take steps to optimize hiring. We will limit automatic sources of hiring, by limiting intake into public service academies by 30 percent, removing job guarantees for private sector teachers, and by putting a sunset clause to existing lists of eligible graduates. We will keep our staffing plans up to date to inform about critical skill requirements. If we are unable to meet our personnel reduction targets, notwithstanding our hiring controls, we will increase targeted redundancies.

Pension reform. Although the recent pension reform addressed long-run issues, short-term pressures remain high, with pensions still increasing as a share of per capita GDP. We therefore will take the following measures effective January 1, 2013, which will yield $€ 5.2$ billion during 2013-14: (i) introduce actuarially-fair rules for lump-sum pensions; (ii) introduce a progressive reduction in monthly pension incomes above $€ 1000$; (iii) eliminate seasonal bonuses for supplementary and main pensions; and (iv) reduce pension increases due to automatic wage promotion for those indexed to wages of special regimes. In addition we will increase the statutory retirement age to 67 and postpone retirement eligibility by twp years in all other cases including early retirement. As a supporting step, to allow identification of pensioners' total pension income, we will finalize the national registry of pensions, and sanction funds that do not deliver the required information to this. 
- Better targeting of social spending. Our reform agenda is expected to yield net savings of 0.6 percent of GDP:

$>$ Health spending. We will continue to reduce public pharmaceutical spending towards 1 percent of GDP, in line with other EU countries. To this end, we will activate the clawback mechanism that maintains outpatient pharmaceutical spending within budget limits. For 2013, we will: (i) revise co-payments; (ii) restrict entry of non-generic drugs into the positive list and expand over-the-counter products; (iii) further reduce the price of off-patent and generic drugs; (iv) reduce average profit margins of pharmacists to 15 percent; (v) reduce hospitals' operational spending (and merge underutilized facilities); (vi) increase contributions by farmers for their healthcare; and (vii) revise the benefit package offered by EOPYY.

$>$ Other social benefit programs. Based on technical assistance advice, we intend to introduce a well designed income-tested system that will reduce spending by 0.2 percent of GDP. Specific measures include: (i) replacing various untargeted family benefits and allowances with one means-tested family benefit program; (ii) reducing special and seasonal unemployment benefits for certain professions and geographic areas; and (iii) increasing the age eligibility and income-testing of social solidarity supplements.

- Improvements in efficiency. Within the central government, we expect to realize savings from reductions of grants to political parties, rationalization of the operating expenditures of social security funds, cuts in lower priority investments, and a general rationalization of the educational system (especially for tertiary institutions). These will yield savings close to $€ 0.7$ billion. We have also cut transfers to public entities outside the general government, alongside their efforts to reduce wasteful investment spending. For local governments, a reduction of $€ 0.2$ billion is planned, for extra-budgetary funds, $€ 0.3$ billion, and for state enterprises $€ 0.3$ billion. These cuts will be supported by stronger fiscal frameworks (discussed below). Finally, to contain contingent risks in the energy sector, we will take measures including raising a solidarity contribution on renewable energy providers and, as a follow up, introduce incentives for cost control of new contracts such as requiring a deposit for existing contracts yet to be connected, and an annual fee on production licenses.

Revenue increasing reforms. Changes will cover both direct and indirect taxes:

> We have submitted to parliament and will enact a reform of the personal, capital, and corporate income taxation regimes by end-December 2012 (meeting a program structural benchmark, with delay). The reform will target higher collections from individuals and entities that have paid a disproportionately low share of the tax burden. To this end, we will broaden the income tax base by abolishing selected tax deductions, allowances, and exemptions. We will shift taxation of the self-employed, farmers, and proprietorships to the corporate tax regime, and increase the fixed charge on companies 
and the self-employed. We will simplify the PIT rate structure to three statutory rates, while introducing a standard tax credit to preserve its progressivity. Finally, we will rationalize the capital income tax to replace the now-diverse levies on capital income with a single flat rate (except for rental income where there will be two rates, and deposit interest, the rate for which will be set lower). These measures, and others, will be designed to yield a net total amount of $€ 1.9$ billion in revenues.

> Several reforms of indirect taxes are designed to yield $€ 0.9$ billion in revenues, including a reduction in the diesel excise subsidy for farmers; a reduction in tax refunds to farmers; an increase in the excise for liquid petroleum gas; and an equalization of the excise on heating oil with that of diesel fuel; restructuring of the taxation of cigarettes in line with EU best practices; and the introduction of a tax on gross gaming receipts. We will consult with the EC/ECB/IMF on any proposed changes in tax rates or bases.

\section{To help cushion the impact of our fiscal adjustment on the most vulnerable, we have strengthened social spending programs. Support for the unemployed will be increased by} $€ 55$ million by 2014, through two new programs (now being piloted): (i) an income-tested benefit scheme that targets long-term unemployed and provides income payable for a year; and (ii) a minimum income guarantee scheme targeting families in areas with difficult socioeconomic profiles. We will also expand our job-training and job matching programs, to be financed where possible by better leveraging available EU structural funds.

\section{We have specified a strategy to fully achieve our $4 \frac{1}{2}$ percent of GDP primary surplus}

target by 2016. We expect revenues to revive once the economy enters its recovery phase, and we anticipate gains from stronger revenue administration (as noted above). Our drive to improve government efficiency will also continue, and we expect to achieve savings from a series of additional spending reforms designed to eliminate government waste (including increasing revenues of state-owned enterprises, contracting out local government services, further restructuring and streamlining of the public administration, and eliminating ineligible pension and social benefits recipients through data cross-checks). To the extent that a fiscal gap remains, there are several strategies that we could pursue to close this, including improving revenue by broadening the tax base through further reduction in exemptions and deductions; extending measures that are expiring; and targeted cuts in current expenditure. There are also opportunities to refocus the investment program for more effective support to growth. We will specify concrete plans for 2015 no later than end-August 2013, when we will formulate a detailed fiscal program for 2014-15 consistent with a primary surplus for 3 percent by 2015 .

33. We are committed to deliver our fiscal targets, but stand ready to adjust if we over or under-perform, or if cyclical conditions change. Following a decision on the final details of the planned direct tax reforms, we will review the fiscal outlook for 2014 and agree with the EC/ECB/IMF on measures needed to close any residual gap for 2014, in the context of the second review of the program. If in future our tax administration reforms, government restructuring, or other fiscal reforms do not deliver the expected dividends and our fiscal targets are thus missed or at risk, we 
will impose expenditure cuts (with a focus on categories of spending where arrears would not arise). In the event of over-performance that is projected to be sustained, we would adjust our budgetary ceilings, with a particular focus on helping low-income earners, and supporting Greece's recovery. In this event, we would use at least 30 percent of the windfall to make our intermediate deficit targets more ambitious to achieve our medium term fiscal objective earlier and accelerate debt reduction.

\section{Fiscal Institutional Reforms}

\section{Strengthening fiscal institutions is a crucial part of the government's reform program.}

Chronic nonpayment of taxes has eroded the fairness of the system, forced high tax rates and additional expenditure rationalization. Meanwhile, the public sector has failed to pay its bills and tax refunds on time, driving up procurement costs and damaging corporate sector liquidity. The reform efforts undertaken to date have yielded only limited results, and we are committed to boosting them, while recognizing that it will inevitably take time for benefits to be fully realized.

35. We are committed to markedly reduce tax evasion. To help accomplish this, we intend to build an independent revenue administration with a modern operating structure and methods. The bullets that follow describe our reform program in more detail. Implementing selected key reforms drawn from these various initiatives will be a prior action for the review (see Annex VI for the details of these upfront reforms).

- We will appoint a new Secretary General of the tax administration. We have passed legislation to define the role and qualifications of the Secretary General. Concerning qualifications, this will be a person with senior management experience, expertise in tax matters, and an impeccable reputation (including a strong tax compliance history). We have also passed interim legislation, and the Minister of Finance will use this to delegate decision making powers to the Secretary General. These powers will include the competence to make operational decisions, direct and control local offices, manage human resources, replace underperforming senior managers, manage the budget of the tax administration, and manage all information with due confidentiality.

- Establishment of independence. Legislation to establish the new semi-autonomous tax agency will be adopted by parliament by end-February 2013 (structural benchmark). This will specify the degree of autonomy, legal powers of the head of the administration, governance framework, the relationship framework with other agencies (including the Financial Intelligence Unit (FIU), the framework for the receipt and use of information by the tax administration (including controls against sharing confidential operational information with the Minister of Finance), accountability, and initial staffing of the organization. The agency will become fully operational in March 2014.

Establishment of key functional units. We have made fully operational key enforcement areas, by: (i) transferring 100 experienced auditors to the large taxpayer unit; and (ii) by establishing one permanent functional unit, with at most two locations, responsible for high- 
wealth individuals (and permanently transferring 50 experienced auditors to it). We will also strengthen collection functions by establishing specialist debt management units in larger local tax offices and allocating at least 10 percent of local staff to this function by end-2012.

Consolidation of tax administration operations. We will close and merge 150 small local tax offices by end-March 2013. By end-June 2013, about 120 functioning offices will remain. The full list of offices to be closed will be published by end-December. We will then centralize and focus audit, filing enforcement, and debt management for small and medium taxpayers in the largest tax offices and on a regional basis. The remaining office network will be customer service centers, with no audit and enforcement functions.

Simplifying record keeping, procedures, and tax legislation. We have repealed the Code of Books and Records and adopted a simpler set of accounting and record keeping rules. To reduce the costs of administration and compliance, by end-May 2013 we will also adopt a new Tax Procedures Code and simplify income tax legislation (structural benchmark). The new code will collect all procedural provisions from the income, VAT, and excise tax laws, and elsewhere; incorporate procedural reforms in all major administrative areas that are necessary to support modern tax administration (e.g., tax filing, audit and penalties, enforcement powers and debt collection); and incorporate a new streamlined administrative dispute resolution process. The simplification will aim to remove ambiguities and excessive cross-references to make it more accessible to taxpayers, and will at the same time adjust numerous aspects of the income tax law (e.g., thin capitalization, EBITDA provisions) to reduce the potential for erosion of the corporate tax base.

Upgrading personnel. We will increase the number of staff devoted to audit by 2,000 by June 2013, by: (i) hiring 200 externally by end-March 2013; and (ii) by admitting staff with audit experience and selecting other qualified staff following interviews. Candidates will, on a selective basis, be subjected to an audit of their assets. The head of the tax administration, once appointed, will set appropriate performance targets for the formal grading of all auditors and staff more generally, and introduce twice-yearly performance assessments. Temporary annual contracts will provide for auditor staff to be terminated upon failure to achieve the targets. A ministerial decision, to be issued in January 2013, will establish appointment and end of tenure procedures for heads of departments, quarterly performance targets, and reporting and assessment procedures.

- Introduction of an effective anti-corruption framework. We will pass legislation to overhaul personnel management (including procedures for rotation of managers in critical offices). By January 2013, we will adopt a code of conduct for the tax administration (including rules about conflicts of interests and declaration of interests), and a system to facilitate transparency and reporting of misconduct, protect whistle blowers, and centralize the decision on disciplinary actions in the internal affairs unit. By end-March 2013, we will define measures to ensure continuous monitoring of the implementation of the code of conduct. The government will publish an anti-corruption plan for the entire public 
administration during Q1 2013, which will also cover in more detail the specific actions for the tax administration.

- Improvements in operating procedures. To allow us to focus our resources where they will yield the greatest dividends and facilitate a risk based approach to auditing, we will legislate by February 2013 the removal of the requirement that all tax declarations for the previous 10 years must be audited (while retaining the right to continue to audit earlier years and the discretion to audit any amount of declarations from these earlier years). We will remove any barriers to using modern audit methods (including barriers to the use of indirect evidence based on presumptive instruments). We will also expand the sources of third party information used in assessments. The tax and AML laws will be revised by February 2013 to: (i) enable the central authority in charge of revenue administration to be informed of all the cases sent by the FIU to local tax offices and to the Corps for the Prosecution of Financial Crime; and (ii) to provide that relevant information on large cases of failure to pay confirmed debt shall be transmitted to the FIU.

- $\quad$ Fiscal identification number. By June 2013, we will require that all ministries which have a fiscal relationship with taxpayers utilize their identification number for financial transactions with them. Looking further ahead, by June 2014, we will introduce a central agency to consolidate and link all of the different identification numbers now employed across various government agencies.

- $\quad$ Accountability to the public: We will launch an easily accessible website by Q1 2013 which will publicize, on a monthly basis, information enabling the public to be informed about the tax debt assessed and recovered. It will include summary statistics on key performance indicators, including on tax evasion cases sent to the FIU and to prosecution by the tax administration.

36. Parallel to our work with the revenue administration we will work to improve the collection of social security contributions. We have finalized a reform plan to modernize collections, with a focus on boosting revenue and recovery, overhauling business processes, performance standards, and inter-agency relationships, and improving the reporting of information that is needed for validating payments and administering benefits. The plan also defines phases for consolidating collections, and integrating them into the tax administration in the long term once its organization and governance have been strengthened. We have established a working group between the Ministry of Labor and the Ministry of Finance to advance the reform. As a first step, by Q1 2013, we will also review penalties and sanctions to ensure that they sufficiently deter evasion and fraud.

37. The government will not introduce any new amnesties or incentive schemes to collect tax arrears or social contributions or extend existing schemes. In this context, the PIT installment scheme introduced in 2012 will remain a one-off arrangement to smooth out the liquidity impact on households of the recently introduced PIT and solidarity charge measures, and we will not extend 
the deadline for the existing social security amnesty. However, we will rationalize debt installment arrangements, including those applying to settlement of tax audits, by tightening the criteria to better reflect ability to pay and targeting only taxpayers under temporary financial stress, but with a good compliance history and reasonable prospects of business viability.

\section{The government is determined to secure tighter control over all general government} spending and to prevent the accumulation of arrears. Our strategy focuses on improved budgeting, stricter controls on expenditure commitments, and better fiscal reporting and monitoring:

- Budgeting. To further streamline and strengthen the preparation process, we will issue a circular, by end-February 2013, regulating the calendar, deadlines, and the role of all institutions in formulating the next MTFS (2014-17) and the 2014 budget; We will also modify the organic budget law to introduce, by August 2013: (i) three year binding expenditure ceilings on a permanent basis as part of the rolling MTFS; and (ii) a provision freezing ex-ante 10 percent of discretionary appropriations per budget line (to be released in the second half of the year conditional upon meeting the fiscal targets). Finally, to ensure independent oversight of the budget process, we will operationalize the parliamentary budget office.

Fiscal framework. We will further develop our fiscal framework to: (i) introduce and make operational a domestic stability pact for local governments; and (ii) establish a system to agree with state-owned enterprises (within the general government definition) on monthly budget targets and on sanctions for non-observance of targets. We will adopt the necessary legislation by end-2012.

- Spending controls. Establishing commitment-based spending controls remains a key milestone toward preventing overspending and accumulation of domestic arrears at different levels of general government. To this end:

$>$ Commitment registers. As a prior action for the review, we will ensure that EOPYY reports from its commitment register through the e-portal for at least two consecutive months (retroactive reports included). By end-2012, we will ensure that commitment registers will be in operation in 90 percent of general government entities (with all commitments recorded into the register at the moment they are made, and all columns of the register complete and reconciled). By March 2013, we will also expand the scope of data captured by the General Accounting Office's e-portal to include the whole expenditure cycle. The Ministry of Finance will continue to monitor the effectiveness of the commitment registers by conducting targeted inspections in the public entities covered by the system.

$>$ General Directorates of Financial Services (GDFS). We have issued a joint circular to implement GDFS in all line ministries and thus unify all financial services functions under 
the recently appointed accounting officer. Moreover, by end-2012 a Ministerial Decision will specify the financial authority and powers of accounting officers in discharging their responsibilities. Thus by end-2012 all line ministries will have established a wellstructured and functional GDFS responsible for managing and supervising all financial functions, including: preparation of the ministry's MTFS and budget proposals; consolidation of budgets of supervised entities; and monitoring and reporting of the ministry's budget execution.

$>$ Fiscal reporting. We remain committed to further improving the quality of fiscal reports. In this regard: (i) we have established a new Social Budget Monitoring Committee to improve the fiscal surveillance of the social security and health sectors, which has begun monthly monitoring of the social budget against fixed quarterly targets. By January 2013 the committee will introduce a comprehensive budget monitoring framework, including improved reporting and control systems of EOPYY and hospital budgets; and (ii) by end-March 2013 we will verify and validate the quality of the detailed fiscal data collected from a recent pilot project before expanding it to all significant general government entities.

\section{The government will clear domestic arrears as quickly as feasible to help improve}

liquidity. The conditions which a government unit must meet to allow funds for arrears clearance to be disbursed will include: for expenditure arrears (i) verification of arrears claims by an audit unit external to the relevant government unit; (ii) establishment by the unit of a fully functioning commitment register; and (iii) reporting of at least three months of consistent data on commitments, payments, and arrears; and, for tax refunds, verification of claims by the audit unit of the Ministry of Finance (the Directorate of Revenue Policy). We will ensure that subvented agencies which meet these conditions can clear their arrears even if their parent agency does not meet the conditions. We will ensure that arrears do not delay the execution of the pharmaceutical spending clawback. Once we achieve the clearance of all verified arrears, we will at that point commence a targeted audit of general government entities' accounts payable, to verify whether arrears remain and to identify any entities that did not properly comply with the conditions set for clearing arrears (completion of the audit will be a structural benchmark for end-December 2013).

40. We will strengthen monitoring of off-balance sheet activities. We will establish by June 2013 a system for monthly financial reporting by state-owned enterprises (SOE) currently outside the general government. Furthermore, we will set conditions under which the right of such SOEs to receive any new general government transfers or loans will be contingent on the adoption of structural measures to restore financial soundness, including through spending cuts and fee increases in consultation with the supervising ministry and the MoF.

\section{We will continue to monitor implementation of our fiscal institutional reforms via} quantified indicators. We propose two new structural benchmarks for end-June and endDecember 2013 covering achievement of revenue administration indicators, including audit targets. We also propose two new structural benchmark for end-June 2013 and end-December 2013 
covering achievement of public financial management indicators, including implementation of spending controls across line entities. The attached Technical Memorandum of Understanding provides details on the quantity targets. In the fifth review of the program, we will focus on overall progress with revenue administration reforms. 


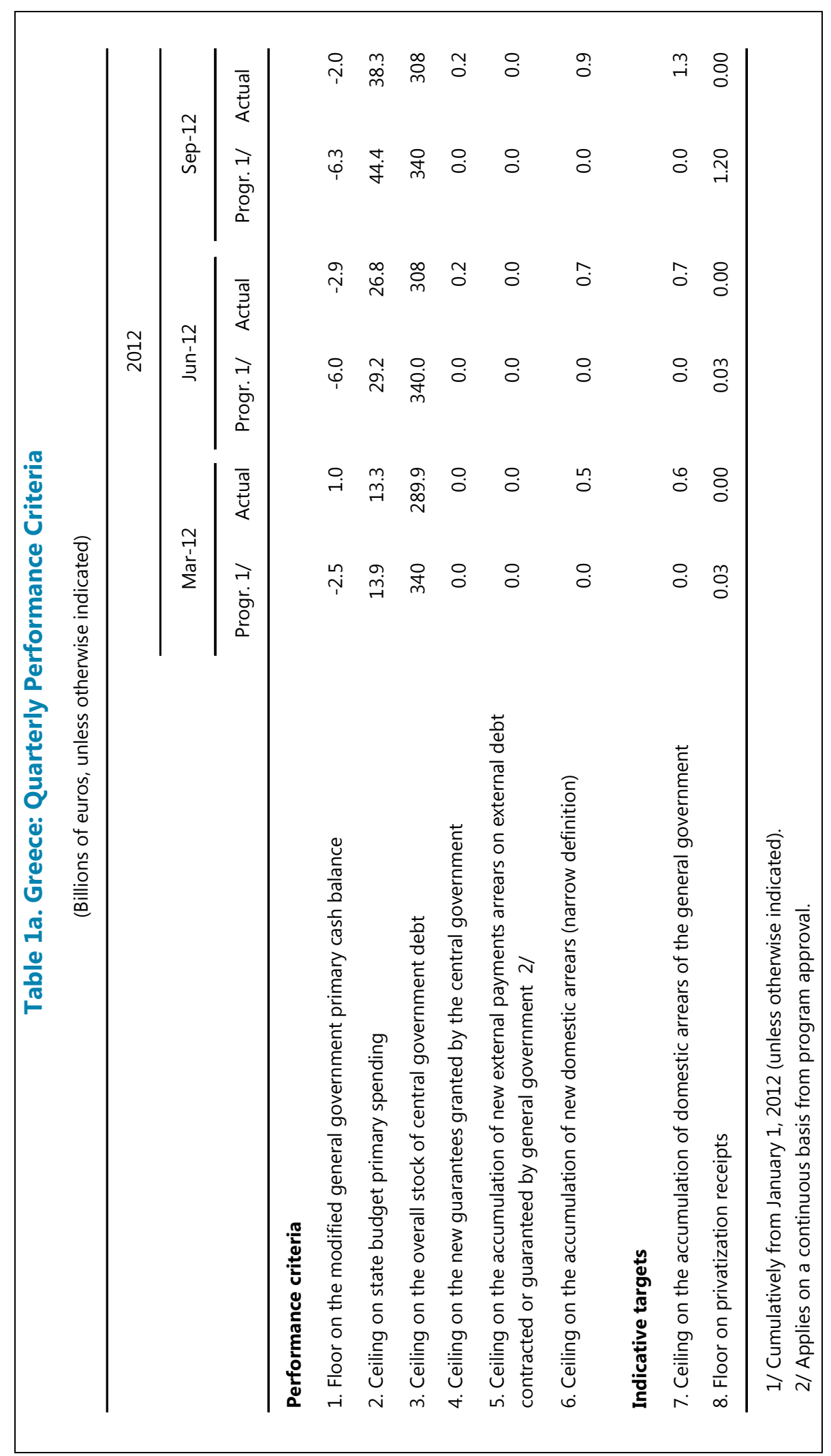




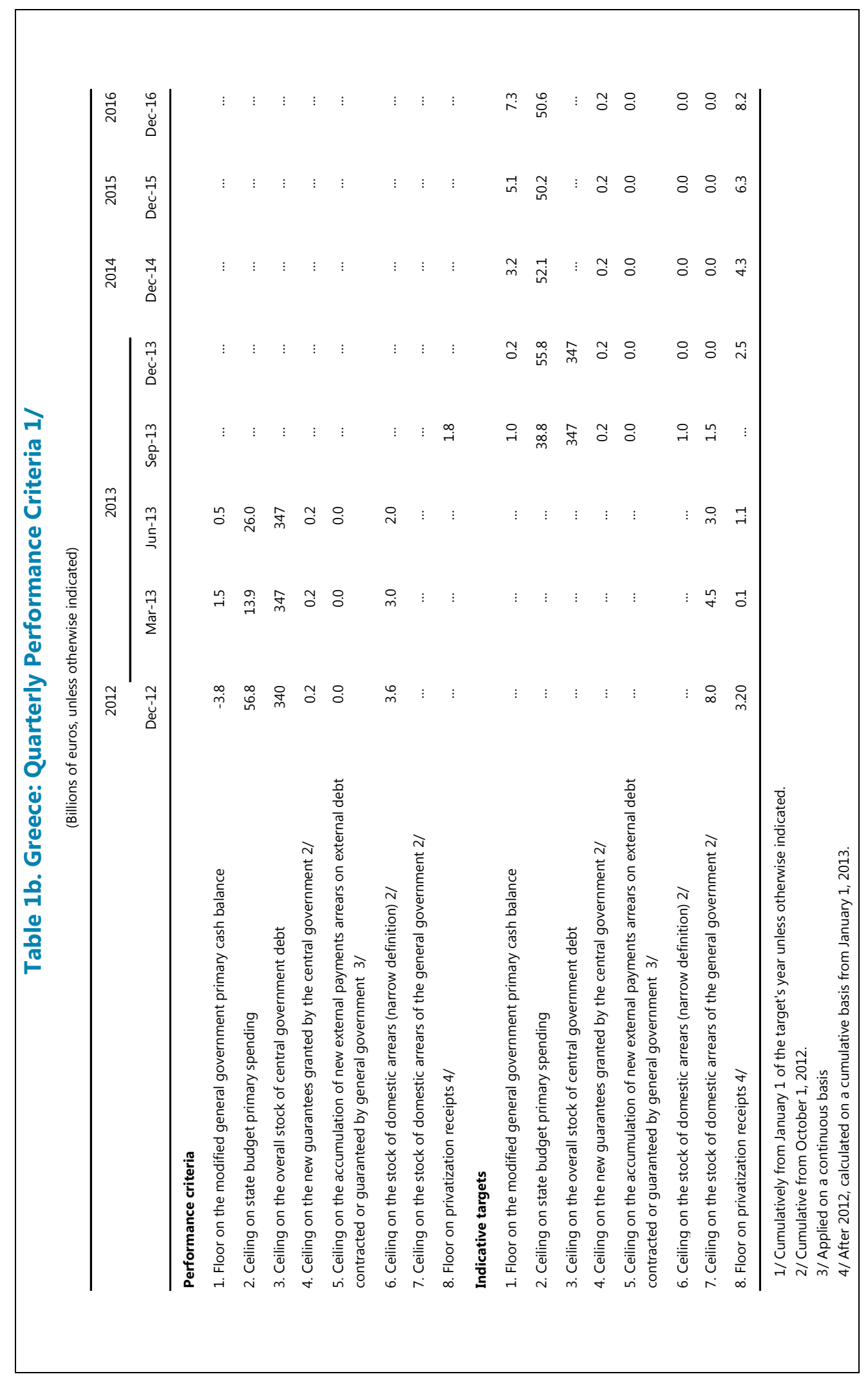




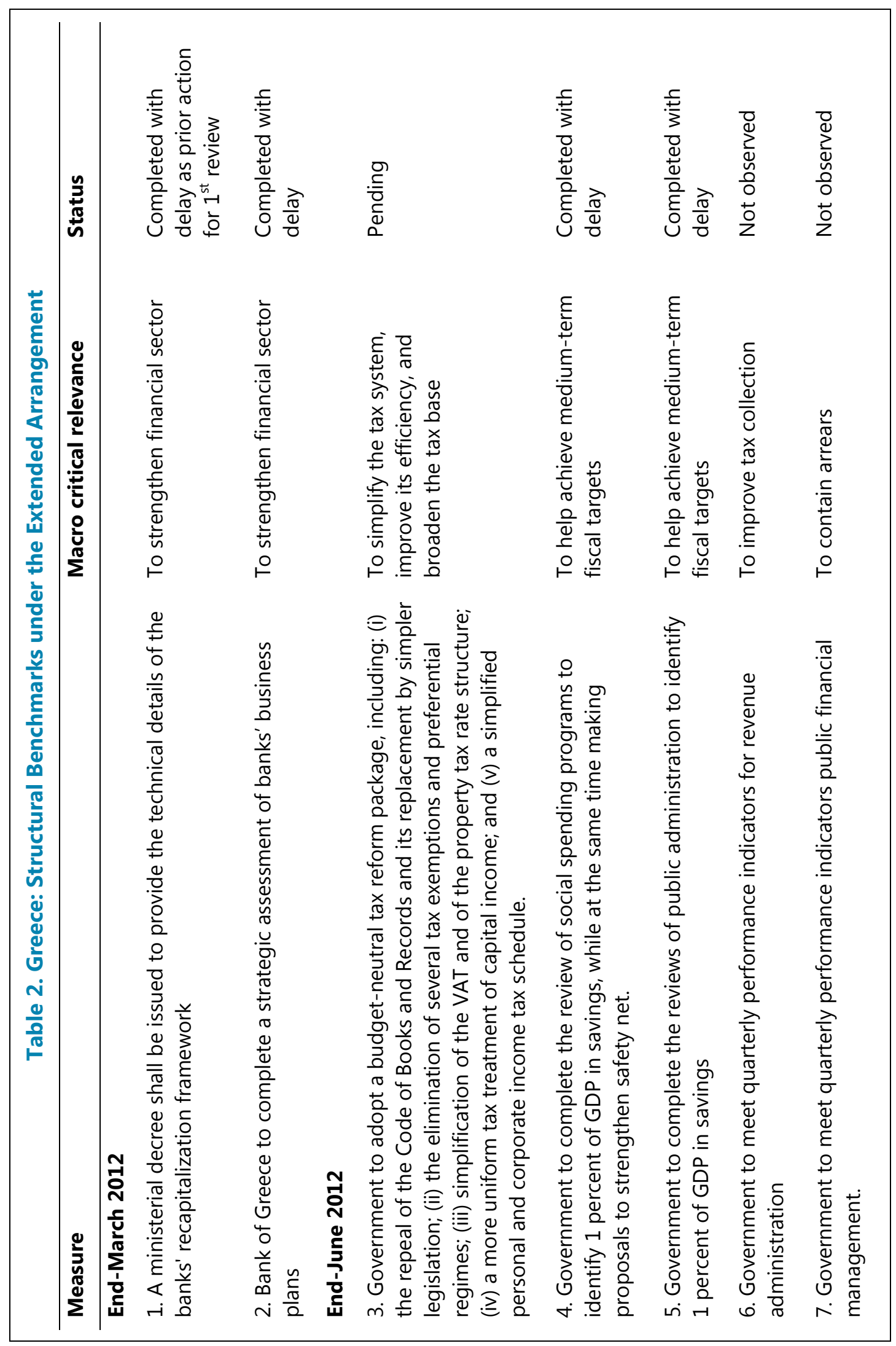




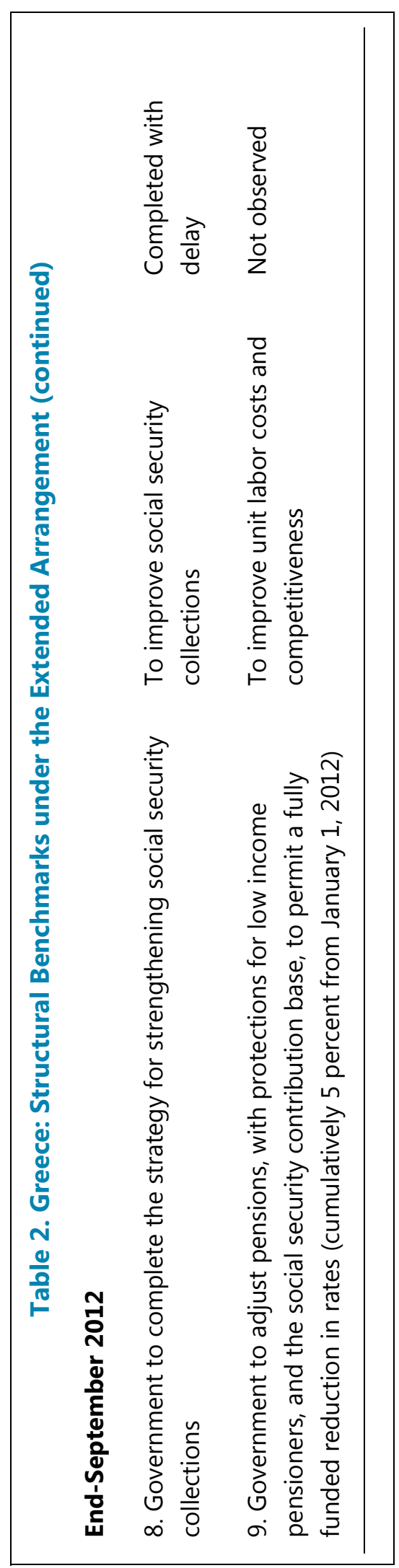




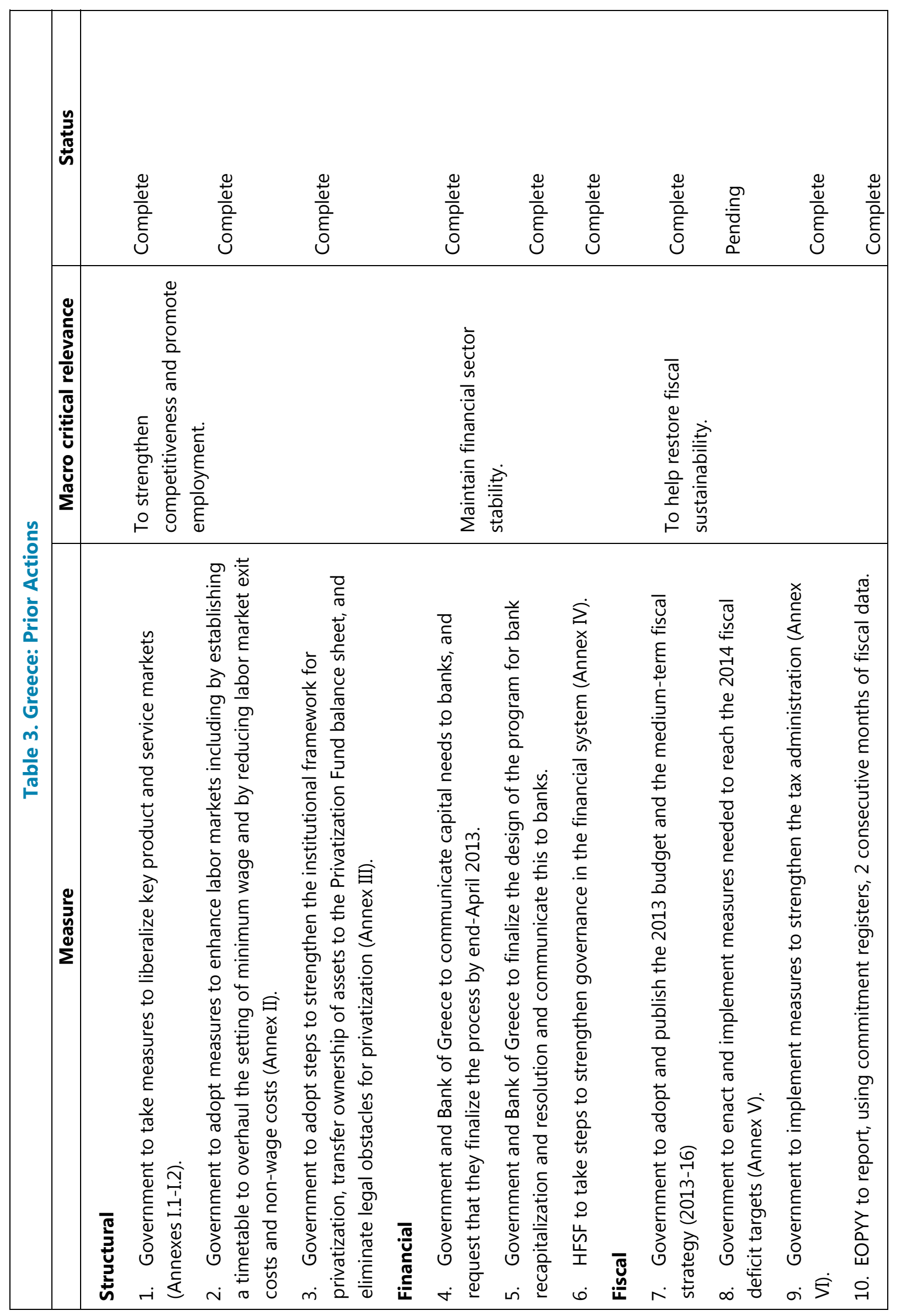




\begin{tabular}{|c|c|c|c|c|c|c|c|c|c|c|c|c|c|}
\hline & 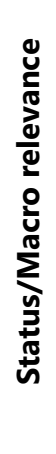 & & 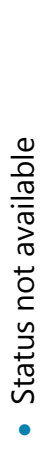 & 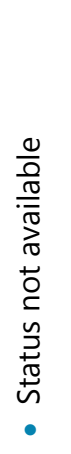 & 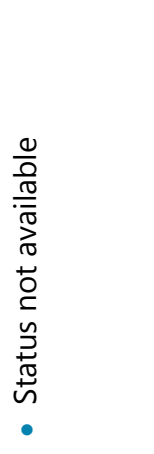 & 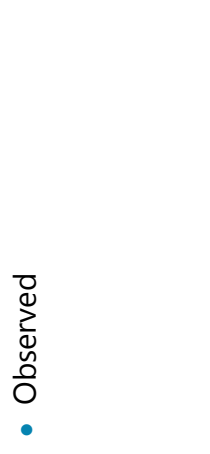 & & 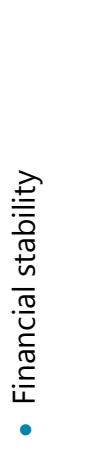 & & 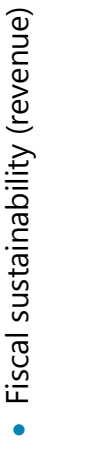 & & & 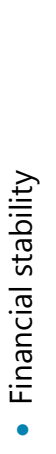 \\
\hline 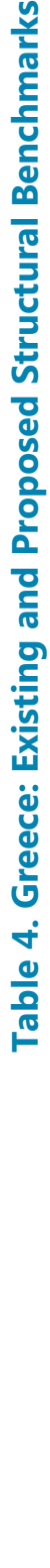 & 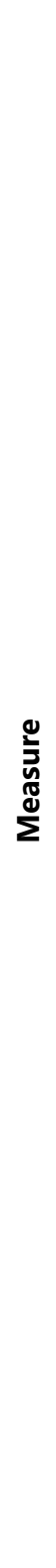 & 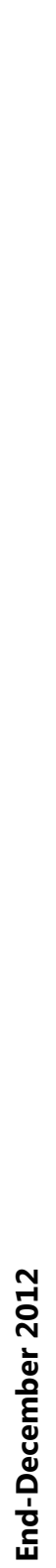 & 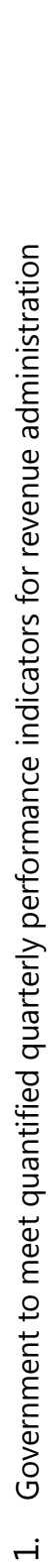 & 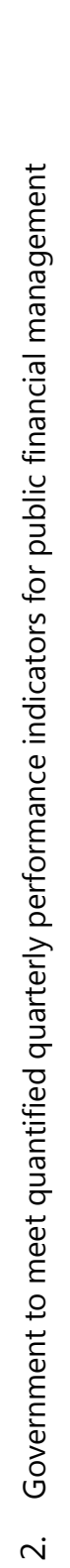 & 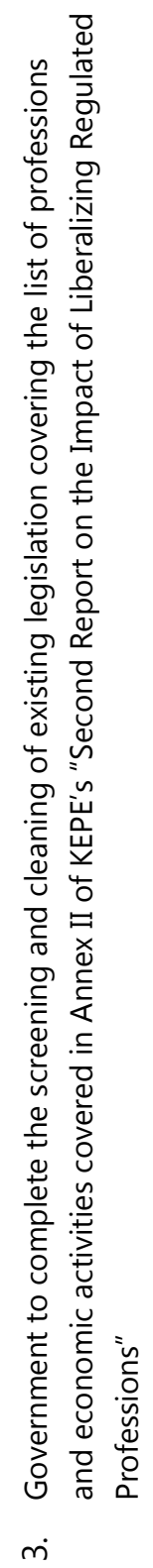 & 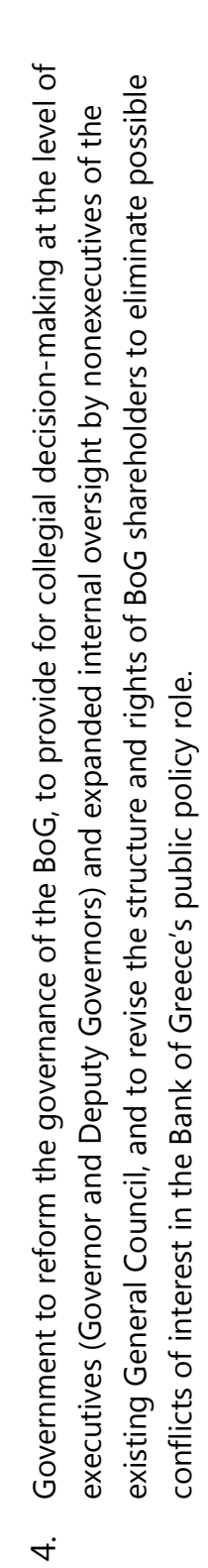 & 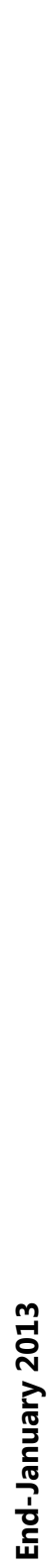 & 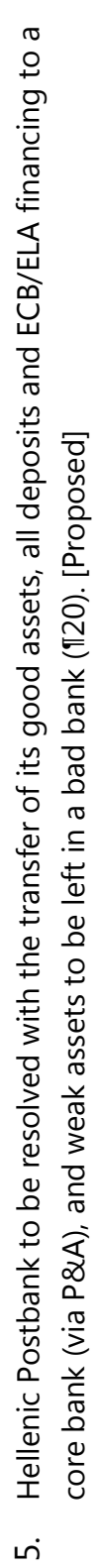 & 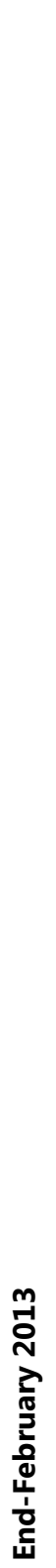 & 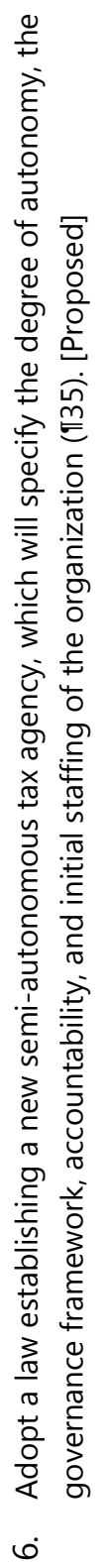 & 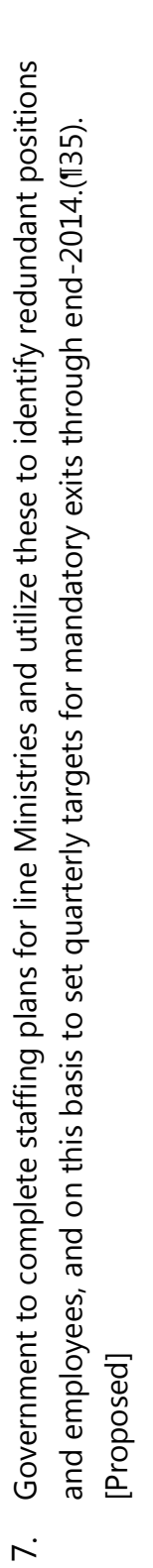 & 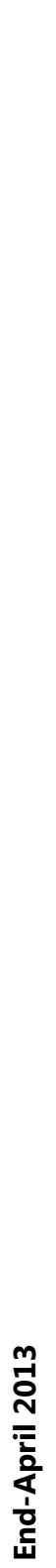 & 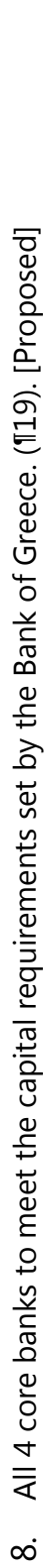 \\
\hline
\end{tabular}




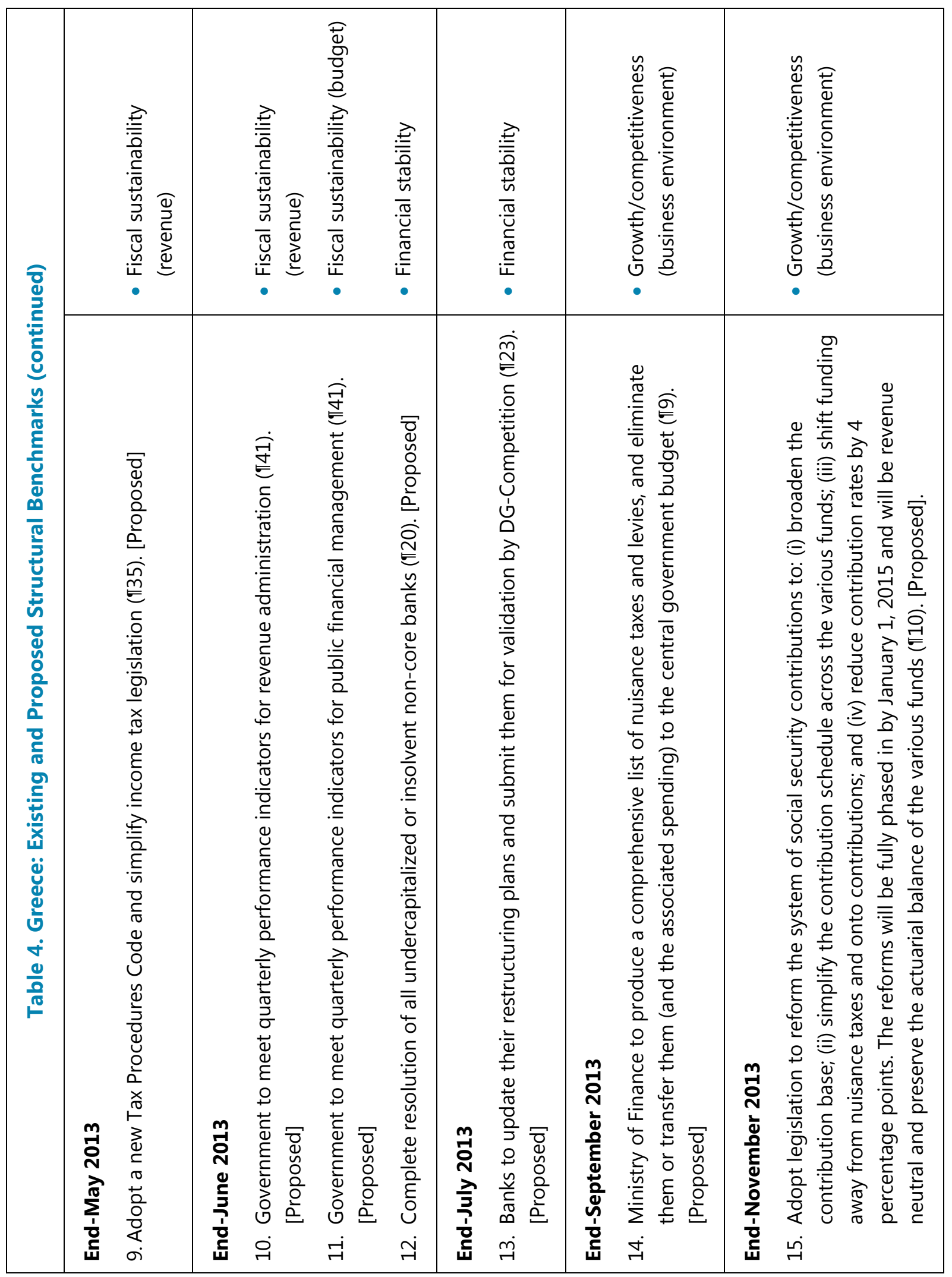




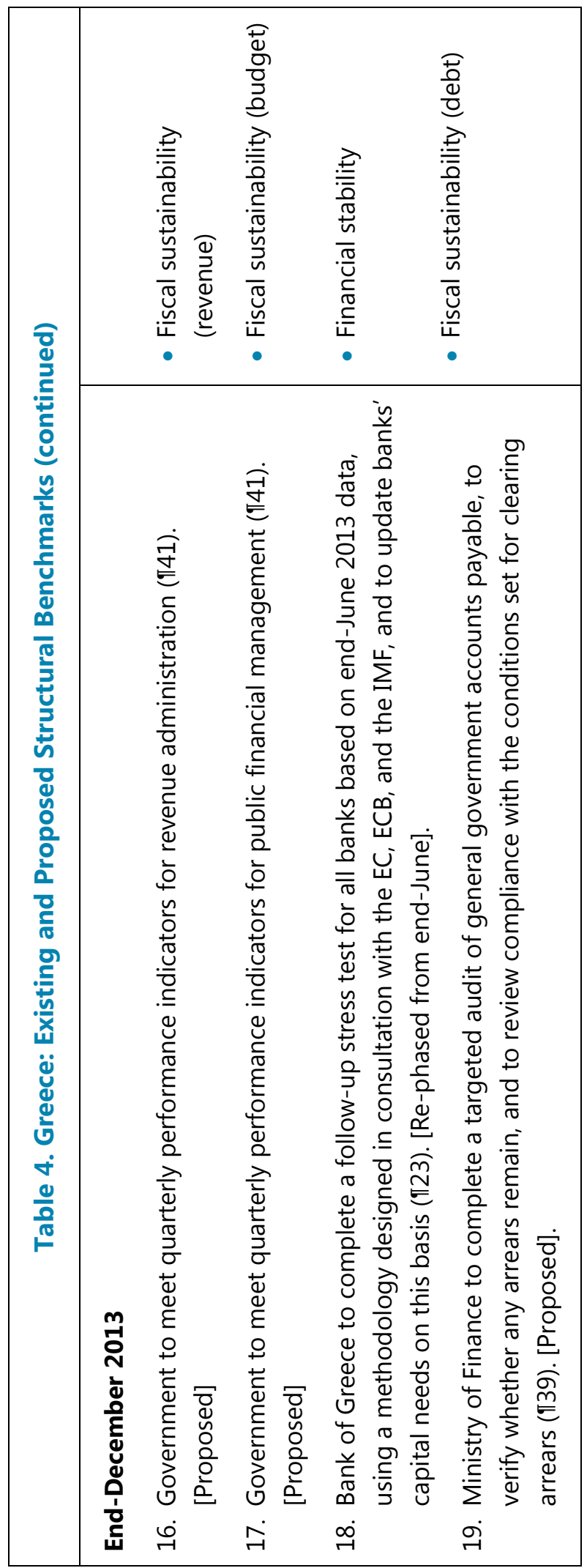




\section{Annex I.1: Product Market Actions}

\section{Fuel market}

- Allow independent gas stations to own and operate tanker trucks above eight tons, and use any tanker size to pick up (imported) fuels, provided that safety standards for the transportation of fuel are respected.

- Allow gas stations to hire a public use tanker for fuel transportation without needing to qualify for their own private use tankers.

- Issue technical specification and set the timetable for the implementation of the input-output measurement system in all fuel stations-with a deadline for Athens and Thessaloniki region of March 2013, other large cities by September 2013, and the rest of the country by March 2014.

- Issue the implementing MD on GPS systems in fuel trucks, to be effective end-2012.

\section{Retail market}

- Allow sale in supermarkets of pre-packaged food products, including meat, seafood, cheese and charcuterie.

- Allow sale in grocery stores of non-food products, including infant milk, tobacco, newspapers, and magazines.

- Allow mixed shops to sell goods other than food, subject to hygiene and food safety standards.

- Eliminate all restrictions on minimum space requirements for sale of food products.

- Allow a delink of the working hours of all employees in establishments (as defined in Law $1037 / 1971$ and related implementing legislation) from opening hours of the establishment.

- Adjust the law to clarify that shift breaks are allowed in all retail establishments (including those with continuous working schedule).

\section{Transportation}

- Remove restrictions on rental of pickup trucks and vans and chauffeur services.

- Allow shuttle services by hotels and tour agencies using small vehicles (less than 12 seats) and tour packages for small vans and off-terrain vehicles. 


\section{Annex I.2 Regulated Professions Actions}

\section{Liberalization of professions/economic activities covered in previous reviews:}

- Stevedores for land operations: Repeal fixed fees for loading/unloading services; remove the Stevedore Work Regulatory Committee from acting as licensing authority.

- Sworn-in valuers: Eliminate minimum fees, the numerous clauses, and the nationality requirement; allow legal entities to practice the profession; and open areas reserved in exclusivity to sworn-in valuers.

- Accountants and tax consultants: Clarify that professional identity cards (IDs) will be issued automatically within three months; and clarify that SAEP is responsible for the recognition of professional qualifications.

- Temporary employment agencies: Abolish the requirement for minimum number of employees. Allow the provision of consulting and training activities.

- Private labor consultancy offices: Allow employees other than the Director to carry out mediation activities; and eliminate the requirement for minimum office space and technical equipment.

- Lawyers. Repeal: mandatory involvement of a lawyer for real estate transactions with immediate effect for the seller, and from 31-12-2013 for the buyer, with a unique thresholds of $€ 80,000$; involvement of a lawyer for contracts for exchange of real estate or gratuitous transactions; the scale of minimum monthly amounts due to private sector lawyers for services rendered with a fixed periodic fee, without prejudice to fee regulations for trainee lawyers and the private minimum wage provided in law 4046/2012.

- Real estate brokers: drop the probationary period for real estate brokers.

- Tourist guides: open the profession to holders of related university degrees.

- Actuaries: discontinue current practice of Hellenic Actuarial Society to determine indirectly the number of successful candidates in the examinations.

\section{Remove the following restrictions on the following professions/businesses:}

- Stevedores at ports: simplify authorization procedures, repeal fixed fees for loading and unloading services; and allow stevedores to be employed under private sector law.

- Customs brokers: repeal minimum fees; lift geographical restrictions, nationality requirements, and age limit; allow legal persons to represent others at customs, allow all natural and legal persons to complete custom formalities without employing services of a custom broker; and remove the need to renew the custom broker license every year.

- Kiosks and cantinas in public buildings: remove restrictions for licenses in favor of particular groups.

- Tourist offices: eliminate prior authorization scheme, minimum office space requirements, and reduce the amount of required bank guarantees.

- Private education establishments: Remove restrictions to private education consistent with the opinion 20/VI/2012 of the Hellenic Competition Commission.

- Private providers of primary care services: Amend sector specific law to eliminate inconsistencies with the 2011 law on professions.

- Press distribution agencies: Remove prior authorization scheme; and expand the number of economic activities carried out.

- Energy inspectors: remove minimum fees, amend sector specific law to repeal minimum fees for energy inspection services, and simplify licensing. 


\section{Annex II: Labor Market Actions}

\section{Setting of minimum wage}

- The government will establish a timetable to reform Greece's minimum wage. Under the new framework:

$>$ The basic minimum wage and the maturity allowances currently set in the national general collective agreement will be replaced by a minimum wage mechanism adopted by parliament following a proposal by the government (to be made after consultation with social partners and other stakeholders and independent experts).

$>$ The system will become effective by end-March 2013. At this point, the statutory minimum wage and maturity allowances will be set equal to the levels agreed at EFF program approval in February 2012 and frozen at these levels. There will be no other allowances.

$>$ The general-national, sectoral, and individual company labor collective agreements will remain free to set higher wages than the statutory level set by the government, and free to set allowances of various types, but these will be binding only for the signatories of the agreements.

$>$ By Q1 2014 the government will assess whether the mechanism is sufficient to address unemployment and improve competitiveness, and whether it is simple enough to be effectively administered by companies.

\section{Nonwage labor costs}

- Concerning dismissal costs, the government will enact legislation to reduce the notification period to 4 months and cap minimum statutory severance pay at 12 months (while preserving the existing link between tenure and minimum severance for tenures below the cap). If the cap has already been surpassed on the date of the reform the amount accrued will be grandfathered, but with the minimum amount beyond 12 months subject to a cap of $€ 2000$ per month. In addition, to secure equal and fair treatment for all employees and occupations, in those where severance costs set by law are in excess of the rule just described, the compensation for severance will be aligned with the rule just described.

- The Ministry of Labor will issue a ministerial decree to reduce administrative burdens by: (i) eliminating the requirement for ex-ante submission of work schedules to labor inspectorates;

(ii) removing preapproval by labor inspectorates of overtime work, itinerary books, the work book of daily employment of construction workers, and applications for leave segmentation (except in the case of preapprovals for underage workers).

- The government will increase work efficiency, within the overall limits of the work week (40 hours) by: (i) applying the general rules on the number of maximum workdays to sectors not now covered by the general rules; (ii) resetting the minimum daily rest to 11 hours; (iii) allowing the consecutive two week leave requirement to be taken anytime during the year in seasonal sectors. 


\section{Annex III. Privatization-Related Actions}

\section{Strengthen institutional framework for privatization}

- Government to present an updated privatization plan to Parliament with the 2012-16 MTFS.

- HRADF to publish a semi-annual update of the Asset Development Plan, which will include a portfolio overview with a description of the privatization assets, a timeline of planned tenders and targeted total receipts for the current and next year.

- Amend paragraph 3 article 16 of the Articles of Association of the HRADF in order to stipulate that the "due cause" required for substituting members of the Board of Directors is defined in particular by the undue suspension or by the intentional compromising of the objectives of the HRADF with acts or omissions of its Board members.

- Amend Law 3986/2011 to require publication of quarterly reports of the HRADF on activities and financial accounts, including a detailed profit and loss statement, cash flow statement, and balance sheet, within 60 days of the end of each quarter.

\section{Transfer ownership of assets to the Privatization Fund}

- Transfer to the portfolio of privatization assets of the HRADF the full and direct ownership (shares or concession rights) of: Egnatia Motorways the regional ports of Elefsina, Lavrio, Igoumenitsa, Alexandropoulos, Volos, Kavala, Corfu, Patras, Heraklion, and Rafina.

- Sign contract between the HRADF and MoF for the use of the voting rights for ELVO.

- Issue an Inter-Ministerial Decision that secures that the proceeds of the sales of the Digital Dividends' Rights are transferred to the HRADF.

- Line ministries/government entities to provide the General Secretariat of Public Property with full access to the inventory of all real estate assets owned by the State.

\section{Eliminate legal obstacles for sale of assets}

- Amend/repeal statutory provisions of companies that diverge from private company law (PPC, OLP and OLTH port authorities, HELPE, EYATH and EYDAP, ports, etc.), including any restrictions on voting rights of private shareholders.

- Launch ESCHADA process (issue environmental study) for Afantou and Kassiopi.

\section{Advisors/Tenders}

- Launch tender for the appointment of advisors, consistent with existing procurement rules, for EAS, ELVO, South Kavala Natural Gas, Trainose. 


\section{Annex IV. Actions to Strengthen Financial Sector Governance}

- The HFSF will complete a due diligence of core banks. This will include a review of governance, such as loans to related parties, asset quality, and risk concentration and any findings of interest to the supervisor will be communicated to the BoG.

- Communicate a terms of reference for bank monitoring trustees to the banks, with instructions for the trustees to begin work no later than mid-January 2013.

- Amend HFSF by-laws to clearly stipulate that the HFSF Board, including the observers, must be informed of all decisions of the core banks having an impact on the HFSF's rights as a shareholder/investor. This information, as soon as received by and through the senior executive of the HFSF, is to be provided a minimum one day in advance of any meeting by the HFSF Board that discusses the bank's decisions. 


\section{Annex V. Fiscal Measures}

\section{Public sector wage bill}

- Adopt legislation to effectively reduce monthly wages of employees under special wage regimes (excluding Christmas, Easter and summer bonuses), effective August 1, 2012, with the following marginal reduction schedule: 2 percent for wages below $€ 1000$; 10 percent for $€ 1000-1500$; 20 percent for $€ 1500-2500$; 30 percent for $€ 2500-4000$; and 35 percent for wages above $€ 4000$.

- Adopt legislation to reduce the State wage bill by $€ 56$ million in 2013 and additional $€ 27$ million in 2014 , including by further reducing fixed-term hires by 10 percent relative to the baseline ( $€ 38$ million in 2013 , $€ 25$ million in 2014), rationalizing the allowances of Members of Parliament, and their staff ( $€ 2$ million in 2013), reducing the wage bill for consultant doctors (by $€ 11$ million in 2013), and introducing a hiring freeze at the Ministry of Citizen Protection.

- Adopt legislation to introduce a new wage grid for parliamentary staff, yielding savings of $€ 14$ million in 2013.

- Adopt legislation to eliminate the public sector seasonal bonuses of employees at the state and local governments, and at legal entities of public and private law, to produce savings of $€ 431$ million in 2013.

- Place 2,000 civil service employees into the mobility and exit scheme, by abolishing positions of certain categories of employees, addressing disciplinary cases (including via outright dismissals), and by abolishing positions associated with closed/merged entities.

- Adopt legislation to abolish all exemptions from the public sector wage grid reform introduced in 2011, excluding HFSF and HRADF, with savings of $€ 8$ million in 2013.

- Adopt legislation to suspend, through 2016, the fiscal bonus of public sector employees, saving $€ 78$ million in 2013.

- Adopt legislation to suspend, through 2016, the performance bonus of public sector employees, saving €214 million in 2013.

- Adopt legislation that: (i) permit the mandatory depositions of teachers from one region to another; (ii) merge school units; and (iii) move administrative staff from the rest of public sector to the Ministry of Education. Upon adoption of the legislation, issue a decision from the joint ministerial committee to reduce the number of non-permanent teaching staff annually to 2,000 teachers with 10 month contracts.

- Issue a decision from the joint ministerial committee to reduce the limit on the number of nonpermanent teachers by 90 percent in university and technical colleges (under the implementation of the plan "Athena").

- Issue a Joint Ministerial Decision to introduce a hiring freeze in the Ministry of Education.

- Adopt legislation to reduce the local government wage bill with savings of $€ 50$ million (to take effect in January 2013).

- Extend the 1:5 hiring rule for the general government through 2016.

- Adopt legislation to align the wage grid for all state-owned enterprises in Chapter A Entities of Private law with the new wage grid for state employees, effective January 1, 2013.

- Adopt legislation that provides mandatory staff transfers (horizontally and vertically) within the general government and from one location in the country to another. 


\section{Annex V. Fiscal Measures (continued)}

- Issue MD to permanently reduce the annual intake into academies by 30 percent, and remove the job guarantee for private teachers. Adopt legislation that all hiring offers from ASEP expire after three years if the actual hiring has not taken place.

- Repeal the provision that provides a full pension to government employees with 20 years of service in case of layoff without losing their social security contributions.

\section{Pensions}

- Increase the retirement age by two years, with effect as of January 1, 2013. The increase will be applied to the statutory retirement age (and any other retirement age for special groups). This increase will not affect the fulfillment of the full pension entitlement at 40 years.

- Reduce, as of January 1, 2013, new lump-sum benefits covering the period back to 2010 by 23 percent on average for different categories of public employees eligible for lump-sum benefits.

- Ministry of Finance and the Ministry of Labor to issue a Joint Ministerial Decision to introduce a pension register by December 2012. The JMD will require that SSFs submit pension data to the entity managing the register by the $10^{\text {th }}$ of each month to aggregate each pensioner's main and supplementary pensions, and note that payments to funds will be withheld for non-compliance with data submission requirements.

- Adopt legislation to implement, using the pension register, the following pension reductions as of January 1, 2013: reduce the overall monthly pension incomes (main and supplementary pensions) per pensioner between $€ 1000-1500$ by 5 percent; $€ 1500-2000$ by 10 percent; $€ 2000-3000$ by 15 percent and above $€ 3000$ by 20 percent (respecting a no re-ranking rule). For retirees formally under special wage regimes, issue a circular applying a cut in their pensions equivalent to the cuts to wages in special wage regimes.

- Further rationalize pensions by: (i) eliminating all seasonal bonuses of supplementary and main pensions (except for the severely disabled); (ii) means-testing pensions for unmarried daughters that were entitled to a pension before the 3865/2010 Law and reducing their pension up to twice the minimum pension; (iii) eliminating special pension benefits of trade unionists; (iv) reducing pensions of elected staff, aiming to achieve savings of $€ 31$ million in 2013; and (v) using completed cross-checks to abolish ineligible pension benefits in 2013.

\section{Social benefits}

- Introduce a length of residence in Greece criterion for the provision of pension benefits for uninsured individuals, aiming to achieve savings (net of income taxes and social security contributions) of $€ 19$ million in 2013 and additional $€ 19$ million in 2014.

- Adopt legislation to replace existing family benefits with a targeted benefit, reducing spending by $€ 86$ million in 2013; and eliminate income tax allowances on 2013 income with the aim of achieving an annual saving of $€ 202$ million in 2013, and a further $€ 177$ million in 2014.

- Issue a Ministerial Decision to restructure the transportation reimbursement scheme for selected categories of patients to equalize the benefits to the 2009 level to save $€ 82$ million.

- Adopt legislation to reduce unemployment benefits targeted to specific geographical areas to achieve savings of $€ 20$ million in 2013. 


\section{Annex V. Fiscal Measures (continued)}

- Adopt legislation to eliminate the special benefits given to people unemployed because of mergers, to generate annual savings of about $€ 10$ million starting from 2013.

- Adopt legislation to target assistance pensions provided by EKAS to persons above 64 years, as to generate savings of $€ 114$ million starting from 2014 .

- Adopt legislation to close two programs for the economic support of farmers, to achieve savings of $€ 25$ million in 2013.

- Adopt legislation introducing new social programs, including:

D Benefits equal to $€ 200$ per month payable for up to 12 months to long-term unemployed who exhaust the full length of unemployment benefit (12 months), provided they do not qualify for other training schemes and have family taxable income up to $€ 10,000$, with expenditure cap of $€ 35$ million.

$>$ A Minimum Income Guarantee scheme applied in two pilot areas of the country with different socioeconomic profiles, with an expenditure cap of $€ 20$ million starting in 2014.

Health

- In order to reduce outpatient pharmaceutical spending the government will:

$>$ Take measures to ensure that the outpatient pharmaceutical expenditure does not exceed $€ 2.88$ billion in 2012 (in commitment terms, net of the structural rebates and of the claw-back). Either: (i) activate and collect the proceeds through the first semester of 2012 from the automatic claw-back mechanism; or (ii) impose the entry-fee onto the positive list; or (iii) apply across-theboard price cuts.

$>$ Set, through a Ministerial decree, a new semi-annual claw-back threshold for 2013, consistent with an annual ceiling of $€ 2.44$ billion for outpatient pharmaceutical spending, including VAT.

$>$ Repeal the current provisions of the law which hamper the collection of the rebate from pharmacies in case of delays in payments on the part of EOPYY effective January 1, 2012.

$>$ Issue a Ministerial Decree to revise the co-payment structure for medicines to exempt from copayment a restricted number of medicines related to specific therapeutic treatments.

$>$ Issue a price bulletin to reduce the price of patented medicines, based on the three EU countries with the lowest prices and re-price medicines now cheaper than $€ 10$, including implementing a 10 percent price reduction in the prices of generics and a 5 percent reduction in the prices of offpatent medicines and issue the quarterly price list update.

$>$ Amend the law to extend the application of the 5 percent rebate on pharmaceutical companies (which exists for hospital medicines) to the products sold in EOPYY pharmacies. (Legislation adopted by Q4 2012).

$>$ Update and publish the positive list of reimbursed medicines to reimburse only the cost effective medicines, especially for chronic diseases, by moving medicines from the positive to the negative and OTC lists and introducing the internal reference price system developed by EOF.

- Modify the form for e-prescriptions to make it compulsory for physicians to prescribe by the international non-proprietary name for an active substance. Brand name prescription is only allowed in limited cases, not exceeding 15 percent of overall prescriptions per doctor. A Ministerial Decision defines the exceptions to INN prescriptions with narrow therapeutic index and known sensitivities of the patient according to international best practices. 


\section{Annex V. Fiscal Measures (continued)}

- Issue a circular to ensure implementation of the ministerial decision requiring that pharmacies substitute prescribed medicines by the lowest-priced product of the same active substance in the reference category by pharmacies (compulsory "generic substitution").

- To improve the current financial situation of EOPYY, adopt legislation with immediate effect to:

$>$ Restrict the benefit package by delisting selected services, establishing utilization caps, and reducing reimbursement prices to save $€ 180$ million in 2013;

$>$ Increase cost-sharing for private care services to at least 30 percent.

$>$ Negotiate price-volume discount agreements with private health care providers, to generate savings of at least $€ 70$ million in 2013;

$>$ Revise the fees for and number of diagnostic and physiotherapy services contracted by EOPYY to private providers with the aim of reducing related costs by at least $€ 80$ million in 2013.

$>$ Introduce a reference price system for reimbursement of medical devices

$>$ Introduce legislation to increase the contributions paid by OGA members to $€ 25$ per month.

- Implement higher copayments for in-patient hospital care and for each prescription and realize savings of $€ 115$ million in 2014.

\section{3 budget ceilings:}

- Set an expenditure ceiling on "operational and other" spending of line ministries (excluding defense, health, and education) so as to reduce it by $€ 203$ million in 2013 and an additional $€ 112$ million in 2014 relative to the 2013 baseline of $€ 3.989$ billion.

- Reduce subsidies to extrabudgetary funds outside the general government and domestic ferry boats by $€ 32$ million in 2013 and additional $€ 66$ million in 2014, relative to the 2013 baseline of $€ 393$ million.

- Set a ceiling to reduce operational costs at higher educational institutions, athletic (federations, sports centers) and cultural (theaters, museums, festivals) institutions to achieve savings of $€ 86$ million in 2013 and $€ 37$ million in 2014.

- Reduce investment spending by $€ 150$ million in 2013 and an additional $€ 150$ million in 2014.

- Set a ceiling on state transfers to SOEs, to save $€ 250$ million in 2013 and additional $€ 123$ million in 2014. Set a ceiling on transfers to STASY to generate savings of $€ 22$ million and $€ 5$ million in 2013 and 2014, respectively.

- Reduce the transfer of Central Autonomous Funds from the State to local governments by $€ 10$ million in 2013 and additional $€ 50$ million in 2014 compared to the baseline of $€ 2,563$ million in 2013 and $€ 2,669$ million in 2014.

- Reduce transfers from the SATA (account for collective decision for local governments) by $€ 40$ million in 2013 and additional $€ 110$ million in 2014 compared to the baseline of $€ 330$ million in 2013 and $€ 330$ million in 2014.

Adopt legislation to amend the spending ceiling of the Green Fund to 2.5 percent of the stock of deposits it holds at the end of the previous year. 


\section{Annex V. Fiscal Measures (continued)}

\section{General government and contingent liabilities:}

- To address contingent fiscal liabilities in the energy sector, pass legislation to (i) introduce a special solidarity surcharge on producer turnover yielding $€ 250$ million a year (effective July 1,2012 , as discussed with the industry); (ii) conclude the Power Purchase Agreement for a photo voltaic station at the price in force at the time that station is put into operation, yielding an average of $€ 150$ million a year; and (iii) adjust the RES Special Levy every six months, beginning on 1 January 2013, to eliminate the RES Account debt by December 2014.

\section{Revenue reforms}

Adopt legislation that:

$>$ Reduces the diesel excise duty subsidy provided to farmers by $€ 130$ million from a baseline subsidy of $€ 163$ million (the rate is reduced from 95 percent to 80 percent of the tax amount);

$>$ Increases the fee for law suits so as to raise an additional $€ 50$ million per year.

$>$ Reforms tobacco excise taxation by raising the per unit tax to $€ 80$ per 1,000 pieces and reducing the ad valorem tax to 20 percent, while minimum tax is raised to $€ 115$ per 1,000 pieces. Rolled tobacco tax will be set at $€ 153 / \mathrm{kg}$.

> Mandates signing an MOU between the government and owners of the merchant fleet to ensure payment of tonnage tax aimed at raising $€ 80$ million in 2013 and $€ 140$ million each year in 2014-16 per year.

$>$ Equalizes the social security earnings ceiling for contributions by raising the ceiling for employees first employed before 1993 to that of employees first employed after 1993, which is $€ 5,543$ per month.

$>$ Increase the excise tax on LPG from $€ 200 / \mathrm{t}$ to $€ 330 / \mathrm{t}$.

$>$ Imposes a 30 percent tax on OPAP's gross gaming revenue for each existing game and a 10 percent withholding tax on player's winnings from the same games-sufficient to raise at least $€ 222$ million, and $€ 88$ million above the baseline, respectively, per year starting in 2013.

Reduce the VAT refund for farmers to 6 percent of turnover.

Submit with the tax reform bill a luxury goods tax effective 2014 with an annual yield of $€ 110$ million. 


\section{Annex VI. Tax Administration Actions}

\section{Independence of the tax administration}

- Adopt legislation to define the role and qualifications of the Secretary General. This should be a person with senior management experience, expertise in tax matters, and an impeccable reputation (including a strong tax compliance history)

- Adopt interim legislation to enable the delegation of powers from the Minister of Finance to the new Secretary General.

\section{Support of core functions}

- Increase the audit capacity of the large taxpayer unit by transferring 100 auditor staff to it.

- Establish a unit responsible for high-wealth individuals and transfer 50 auditor staff to it.

\section{Anti-corruption strategy}

- Adopt legislation to implement a simpler set of accounting and record keeping rules and repeal the Code of Books and Records.

- Adopt legislation to overhaul personnel management (covering procedures for rotation of managers in critical tax offices) 


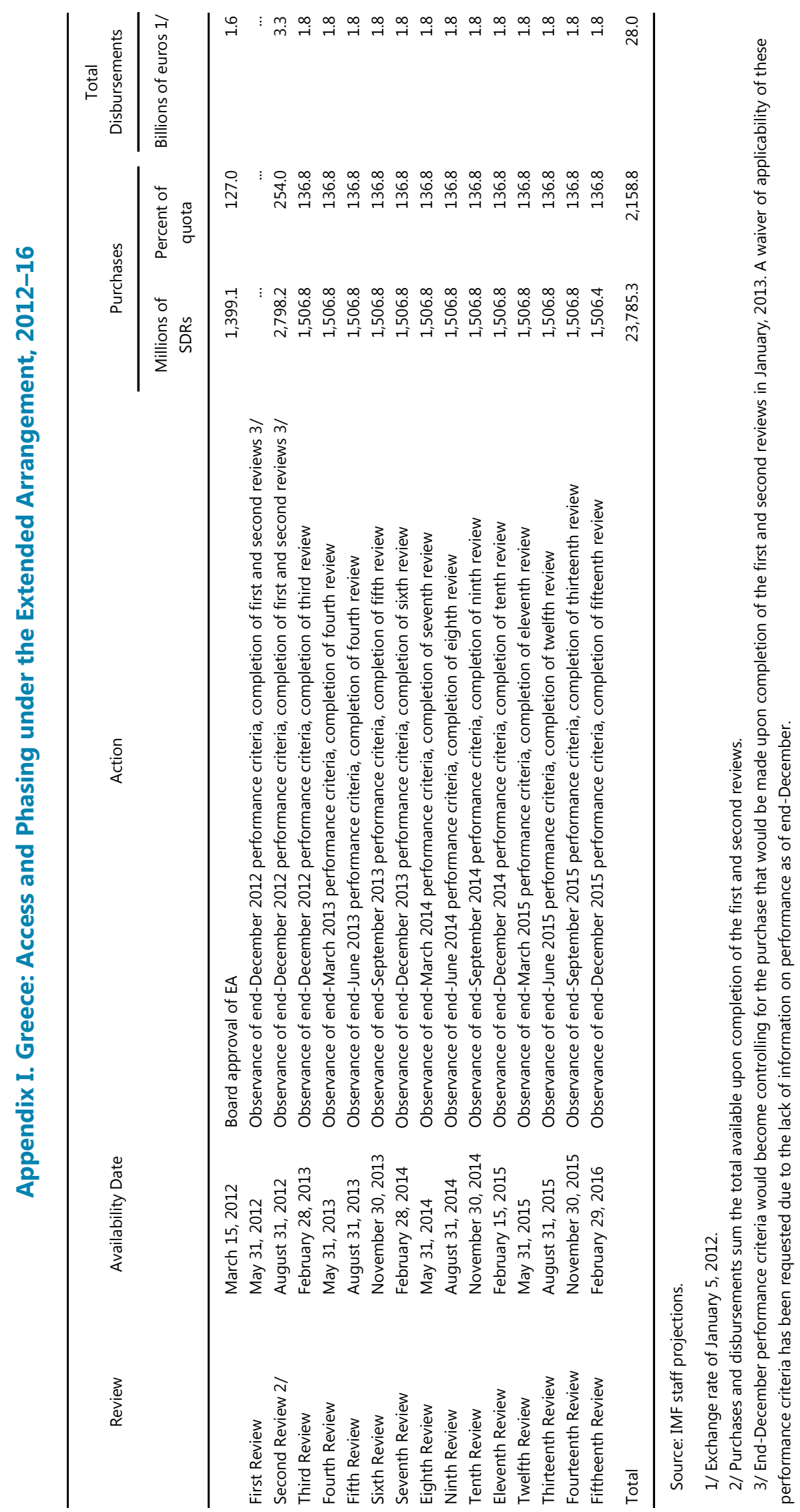




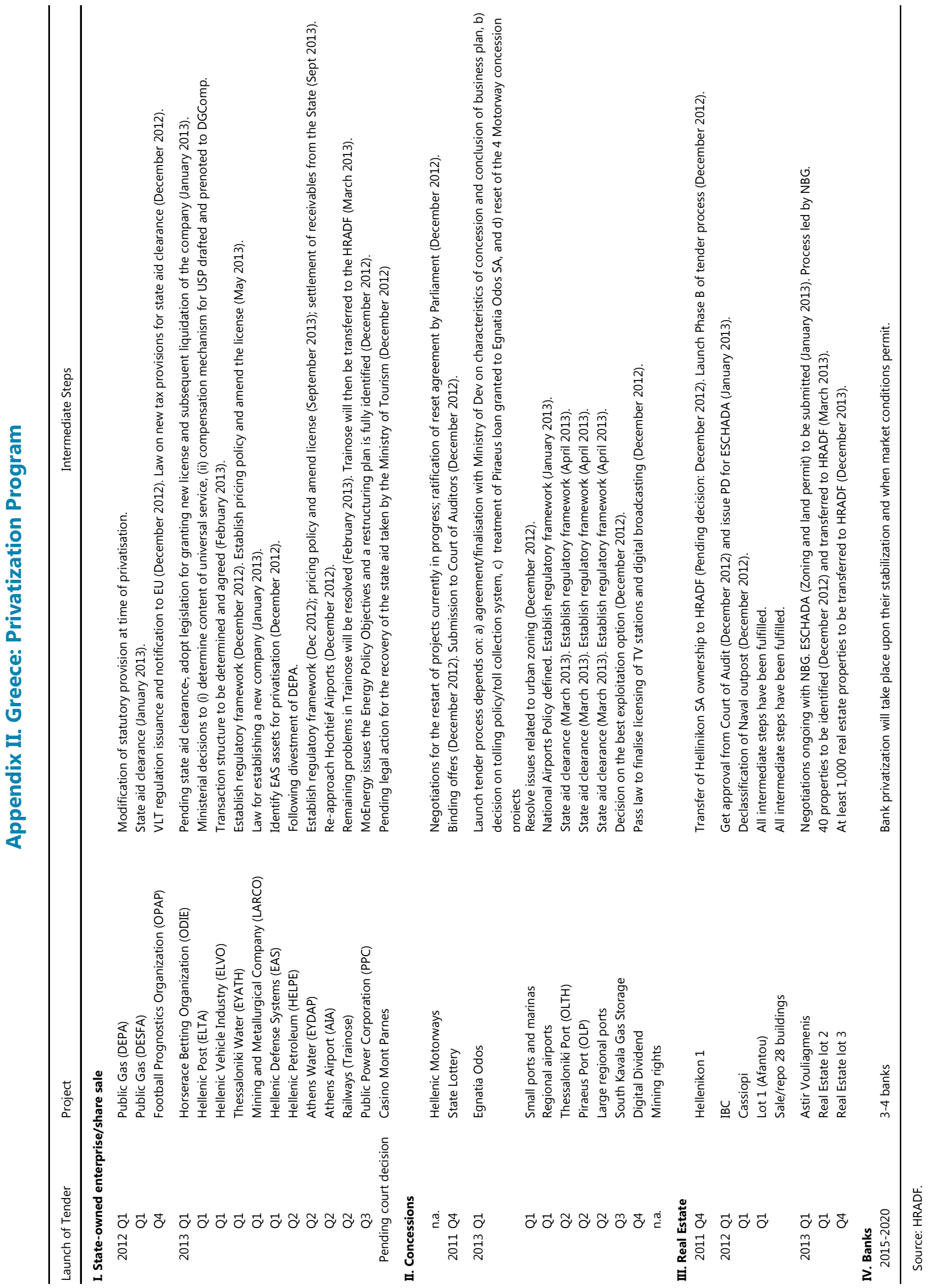




\section{APPENDIX V: TECHNICAL MEMORANDUM OF UNDERSTANDING}

December 21, 2012

1. This Technical Memorandum of Understanding (TMU) sets out the understandings regarding the definitions of the indicators subject to quantitative targets (performance criteria and indicative targets), specified in the tables annexed to the Memorandum of Economic and Financial Policies. It also describes the methods to be used in assessing the program performance and the information requirements to ensure adequate monitoring of the targets. We will provide the European Commission, ECB and the International Monetary Fund with the necessary information for program monitoring.

2. For program purposes, all foreign currency-related assets, liabilities, and flows will be evaluated at "program exchange rates" as defined below, with the exception of the items affecting government fiscal balances, which will be measured at current exchange rates. The program exchange rates are those that prevailed on January 31, 2012. In particular, the exchange rates for the purposes of the program are set: $€ 1=1.3176$ U.S. dollar, $€ 1=100.63$ Japanese yen, and $€ 1.1772=1$ SDR.

\section{General Government}

3. Definition: For the purposes of the program, the general government includes:

- The central government. This includes:

$>$ The entities covered under the State Budget as defined in Chapter 2 of the Law 2362/1995 as being modified by Law 3871/2010 regarding "Public Accounting, Auditing of Government Expenditures and Other Regulations," and other entities belonging to the budgetary central government.

$>$ Other entities or extra-budgetary funds (EBFs) not part of the State budget, but which are, under European System of Accounts (ESA95) rules ("ESA95 Manual on Government Deficit and Debt'), classified under central government. This includes the Green Fund and the HRADF.

- The following state enterprises and organizations included by the National Statistical Service (ELSTAT) under the definition of central government (ATTIKO METRO, OSY, ELGA, HELLENIC DEFENCE SYSTEMS S.A., OSE, TRAINOSE, ERT, ELECTROMECHANICA KYMI LTD, OPEKEPE, KEELPNO, EOT, GAIAOSE, ERGOSE, INFORMATION SOCIETY IN GREECE, Unit for the Organization and Management of Development Projects S.A.). 
- Local government comprising municipalities, prefectures, and regional governments including their basic and special budgets, including all agencies and institutions attached thereto, which are classified as local governments according to ESA 95.

- Social security funds comprising all funds that are established as social security funds in the registry of ELSTAT.

- Other extra budgetary entities included by ELSTAT under general government, which are not yet counted under central government.

- $\quad$ This definition of general (central) government also includes any new funds, or other special budgetary and extra budgetary programs that may be created during the program period to carry out operations of a fiscal nature. The government will inform IMF, European Commission and ECB staff of the creation of any such new funds, programs, or entities immediately.

- Supporting material: The Ministry of Finance (MoF) will provide to the European Commission, ECB and IMF detailed information on monthly revenues and expenditures, domestic and foreign debt redemptions, new domestic and foreign debt issuance, change in the domestic and foreign cash balances of the central government at the central bank of Greece, all other sources of financing including capital transactions, and arrears of the general government. The Ministry of Finance, in collaboration with the Ministry of Interior, will provide monthly data on revenues and expenditures for local governments, as collected in the Ministry databank. The Minister of Finance, in collaboration with the Ministry of Labor and Ministry of Health, will provide monthly data on revenues and expenditures in main social security funds (including IKA, OGA, OAEE, OAED) and the central healthcare fund (EOPYY). The Bank of Greece will provide detailed monthly data on assets and liabilities of local authorities, social security funds, the Green Fund (and other extra-budgetary funds), and state enterprises included in the definition of general government in line with monetary survey data. Data will be provided within four weeks after the closing of each month.

\section{Quantitative and Continuous Performance Criteria, Indicative TARgets, ANd Continuous Performance Criteria: DEFINITIONS AND REPORTING STANDARDS}

\section{A. Floor on the Modified General Government Primary Cash Balance (Performance Criterion)}

4. Definition: The modified general government primary cash balance (MGGPCB) is defined as the modified general government cash balance (MGGCB) minus interest payments by the state budget. The MGGCB is defined as the sum of the cash balances of the ordinary state budget, the cash balance of the public investment budget, the change in net financial assets of 
local government, the change in net financial assets of social security, the change in net financial assets of the Green Fund, the change in net financial assets of reclassified public enterprises (RPEs) minus guarantees called to entities within the general government and the spending by the HRADF. Privatization receipts, as defined below and the proceeds from the sale of land and buildings will be excluded from cash receipts. Net lending operations by the state budget will be recorded as cash expenditures.

The cash balance of the ordinary state budget. The cash balance of the ordinary state budget will be measured from above the line, based on: (i) gross ordinary budget revenues (recurrent revenue plus non-recurrent revenue, minus tax refunds (excluding any payments for the clearance of tax refunds in arrears); minus (ii) ordinary budget expenditures as published monthly on the official website of the General Accounting Office of the Ministry of Finance, and in line with the corresponding line items established in the ordinary state budget. Ordinary budget expenditures will exclude amortization payments, but include: salaries and pensions; grants to social security funds, medical care and social protection; operational and other expenditure; returned resources; payments in exchange of claims of insurance fund for the personnel working in the Public Electricity Company; the reserve, interest payments; transfers for the settlement of past debt, payments for military equipment procurement on a cash basis; NATO expenses, capital transfers to social security funds or other entities by bonds; and called guarantees where the state or central government assumes payments on behalf of entities outside of the general government.

The cash balance of the public investment budget. The cash balance of the public investment budget will be measured from above the line, based on investment budget revenues minus investment budget expenditures of the investment state budget as published monthly on the official website of the General Accounting Office of the Ministry of Finance, and in line with the corresponding line items established in the investment state budget.

$>$ The change in net financial assets of local governments is defined on a transactions basis, as the change in the total of financial assets minus financial liabilities of local authorities adjusted for valuation changes by the Bank of Greece.

$>$ Financial assets include (but are not limited to) deposits of local governments in the Bank of Greece and deposits of local governments in domestic credit institutions. Deposits will be measured at face value excluding accrued interest in line with recording for monetary survey data.

> Financial liabilities include (but are not limited to) short- and long-term loans from domestic credit institutions to local governments, measured at face value, consistent with recording for monetary survey data. 
$>$ The change in net financial assets of social security funds is defined on a transactions basis, as the change in the total of financial assets minus financial liabilities of social security funds, adjusted for valuation changes by the Bank of Greece.

$>$ Financial assets include

- Deposits of social security funds in the Bank of Greece and deposits of social security funds in the domestic credit institutions and deposits held either directly or indirectly through the IKA mutual fund. Deposits are measured at face value excluding accrued interest, consistent with reporting requirements for monetary survey data.

- Holdings of shares quoted on the Athens Stock Exchange held by social security funds either directly or indirectly through the IKA mutual fund.

- Direct or indirect holdings of Mutual Fund units issued by Greek management companies (other than the IKA mutual fund).

- Holdings of central government bonds, including short and long-term securities issued domestically, long-term securities issued abroad operated from Bank of Greece accounts, and indirect holdings through the IKA mutual fund. Holdings will be measured at nominal value.

- Holdings of bonds issued abroad and other foreign assets.

Financial liabilities include the short and long term loans from domestic credit institutions to the social security funds, measured consistently with monetary survey data.

$>$ The change in net financial assets of the Green Fund is defined on a transactions basis, as the change in the total of financial assets minus financial liabilities of the Green Fund, adjusted for valuation changes by the Bank of Greece.

$>$ Financial assets include

- Deposits of the Green Fund in the Bank of Greece and in domestic credit institutions. Deposits will be measured at face value excluding accrued interest in line with recording for monetary survey data.

- Holdings of shares, held by the Green Fund, quoted on the Athens stock exchange.

- Holdings of Mutual Fund units issued by Greek management companies.

- Holdings of central government bonds. 
- Holdings of other bonds issued abroad.

> Financial liabilities include the short and long term loans from the domestic credit institutions to the Green Fund, measured consistently with monetary survey data, or other lending from the Bank of Greece.

The change in net financial assets of reclassified public enterprises (RPEs) is defined on a transactions basis, as the change in the total of financial assets minus financial liabilities of RPEs, adjusted for valuation, minus the amount of guarantees called from entities which are consolidated within the general government. RPEs will include the following organizations: ELGA, KEELPNO, OPEKEPE (excluding the account ELEGEP), EOT, ATTIKO METRO, HELLENIC DEFENCE SYSTEMS S.A., ERT, TRAINOSE, ERGOSE, GAIAOSE, OSY, ELECTROMECHANICA KYMI LTD, INFORMATION SOCIETY IN GREECE, MANAGEMENT ORGANISATION UNIT, and OSE.

$>$ Financial assets include

- Deposits of RPEs in the Bank of Greece and deposits of RPEs in the credit institutions (domestic and foreign). Deposits will be measured at face value excluding accrued interest.

- Holdings of shares, held by RPEs quoted on the Athens Stock Exchange.

- Holdings of Mutual Fund units issued by Greek management companies.

- Holdings of central government bonds.

- Holdings of other bonds issued abroad.

Financial liabilities include the short and long term loans from the domestic credit institutions to RPEs, measured consistently with monetary survey data. They also include short and long term loans from the foreign banking system, as well as loans from the EIB or other official lenders, as measured by the difference between new loans granted to these entities (as approved by the GAO in line with the Fiscal Responsibility Act) and amortization of these loans through called guarantees of the government or amortization of these loans made by actual payments by the companies themselves, upon monitoring and information provided by the General Accounting Office (D25).

The expenditures of the HRADF are defined from below the line as the change in deposits of the HRADF net of deposit changes due to borrowing for securitization purposes that are remitted to the central government as privatization receipts. Changes in net deposits of the HRADF and borrowing are to be measured from the monetary survey data for data on borrowing and on deposits held in commercial banks. For 
deposits held at the central bank, net deposits are measured directly from the Bank of Greece. Remittance of privatization proceeds to the state will be measured from the inflows into the Treasury Single Account.

\section{Other provisions for the purposes of the program.}

> The MGGPCB will also exclude all transfers related to the Eurogroup decisions of February 21, 2012 and November 26, 2012 in regards to income of euro area national central banks, including the BoG, stemming from their investment portfolio holdings of Greek government bonds.

$>$ Receipts from privatization are excluded from cash general government revenue receipts. However, for the entire program period where this is applicable, sales of those gaming licenses, telecom licenses, sales of aircrafts, and extension of the airport concession that were established in the context of the May 2010 SBA program or the 2011 budget (Second Review) discussions will be recorded as cash revenue receipts and taken into account for the MGGPCB criterion, irrespective of whether the realized proceeds accrue to the privatization agency or not.

$>$ The primary expenditure of the central government excludes payments related to bank support. Transactions that may be excluded from the balance include loans to financial institutions and investments in equity of financial institutions (requited recapitalization); unrequited recapitalization; purchases of troubled assets, and operations related to the FSF. Any financial operation by central government to support banks, including the issuance of guarantees or provision of liquidity, will be immediately reported to IMF, European Commission, and ECB staff.

$>$ The primary revenue of the central government will exclude any cash payments from loss-making banks beyond those which would accrue from the ELA guarantee fee structure existing on November 30, 2012 (25 basis points).

> Capital transfers to social security funds or other entities by bonds shall exclude bond issuance for settlement of end-2009 health related arrears, and the settlement related to the judiciary liabilities.

\section{Supporting material.}

- Data on cash balances of the ordinary and state budgets will be provided to the European Commission, ECB and IMF by the General Accounting Office in the Ministry of Finance within three weeks after the end of each month. Data will include detailed information on revenue and expenditure items, in line with monthly reports published on the official website of the Ministry of Finance. Data will also include data on capital transfers to social security funds or other entities in bonds and called guarantees. 
- Data on net financial assets of local authorities and social security funds, extra-budgetary funds including the Green Fund, AKAGE, and reclassified public enterprises will be provided to the IMF, European Commission and ECB by the GAO in cooperation with the Statistics Department of the Bank of Greece within four weeks after the end of each month.

\section{B. Ceiling of State Budget Primary Spending (Performance Criterion)}

6. Definition. The state budget primary spending consists of state budget spending (spending of the ordinary state budget plus spending of the public investment budget) minus interest expenditures paid by the state budget, minus any arrears payments made. Ordinary state budget spending includes called guarantees to entities inside the general government (as opposed to the definition of the modified general government primary cash balance criterion above that excludes this spending item). Primary expenditure of the central government that is monitored for the Performance Criterion excludes any cash payments related to bank restructuring, when carried out under the program's banking sector restructuring strategy. Costs that may be excluded from the balance include loans to financial institutions and investments in equity of listed and non-listed financial institutions (requited recapitalization); unrequited recapitalization; and purchase of troubled assets. However, any financial operation by central or general government to support banks, including the issuance of guarantees or provision of liquidity, will be immediately reported to European Commission, ECB and IMF staff.

7. Supporting material. The General Accounting Office of the Ministry of Finance will provide monthly expenditure data of the ordinary and investment state budget, as defined above.

\section{Ceiling on the Stock of Domestic Arrears (narrow definition) (Performance Criterion)}

8. Definition. For the purpose of the program, domestic arrears (narrow definition) are defined as: (i) unpaid invoices of line ministries and hospitals that are 90 days past their due date; plus (ii) tax refunds for which a refund document "AFEK" has been issued and cleared but have not been repaid to the taxpayer. In case no due date is specified on the supplier contract, an unpaid commitment is considered to be in arrears 90 days after the initiation of the invoice. The stock of arrears excludes: (i) the arrears which are being accumulated by the Civil Servants' Welfare Fund; and (ii) hospital arrears to pharmaceutical companies which were incurred by end2009 ( $€ 113$ million as of November 30, 2012). Beginning July 1, 2013, the definition will include the change in the stock of tax refund claims that have not been assessed within 90 days (cumulative from July 1); and beginning January 1, 2014, it will include all tax refund claims that have not been assessed within 90 days. In both cases refund claims that are under legal dispute will be excluded.

9. Supporting material. Monthly data on arrears of public hospitals (NHS hospitals) will be provided by the Ministry of Health, and arrears of line ministries by the Ministry of Finance within 
four weeks after the end of each month. The Ministry of Finance will publish this information on the Ministry of Finance website. The expenditure arrears data will be based on survey data, until data from commitment registers are assessed by the IMF, European Commission, and ECB staff to provide comprehensive and reliable information. These reports will also include data on accounts payable overdue by $0-30,31-60$, and $61-90$ days for the central government (line ministries and Decentralized Prefectures) based on the commitment registers. Tax refund arrears data will be based on information provided by General Secretariat for Information Systems and General Secretariat for Tax and Customs. The Ministry of Finance will also provide a monthly table on tax refund arrears as defined above (with AFEK issued) as well as on full tax refund accounts payable that include any refund claims for which AFEK has not been issued (and showing those that have not been assessed after 90 days).

\section{Ceiling on the Stock of Domestic Arrears of the General Government (Indicative Target)}

10. Definition. For the purpose of the program, domestic arrears of the general government are defined as: (i) unpaid invoices of general government entities that are 90 days past their due date; plus (ii) tax refunds for which a refund document "AFEK" has been issued and cleared but have not been repaid to the taxpayer. In case no due date is specified on the supplier contract, an unpaid commitment is considered to be in arrears 90 days after the initiation of the invoice. The stock of all general government arrears excludes: (i) the arrears which are being accumulated by the Civil Servants' Welfare Fund; and (ii) hospital arrears to pharmaceutical companies which were incurred by end-2009 ( $€ 113$ million as of November 30, 2012). Beginning July 1, 2013, the definition will include the change in the stock of tax refund claims that have not been assessed within 90 days (cumulative from July 1); and beginning January 1, 2014, it will include all tax refund claims that have not been assessed within 90 days. In both cases refund claims that are under legal dispute will be excluded.

11. Supporting material. The Ministry of Finance will provide consistent data on monthly expenditure and tax refund arrears of the general government, as defined above within four weeks after the end of each month, and publish this information on the Ministry of Finance website. The expenditure arrears data will be based on survey data, until data from commitment registers are assessed by the IMF, European Commission, and ECB staff to provide comprehensive and reliable information. These reports will also include data on accounts payable overdue by $0-30,31-60$, and $61-90$ days for the central government (line ministries and Decentralized Prefectures), based on the commitment registers. Tax refund arrears data will be based on information provided by General Secretariat for Information Systems and General Secretariat for Tax and Customs. 


\section{E. Ceiling on the Overall Stock of Central Government Debt (Performance Criterion)}

12. Definition. The overall stock of central government debt will refer to ESA95 central government debt, which includes the state debt, debts of extra budgetary funds and public enterprises that are consolidated into the central government, and other ESA 95 adjustments. Holdings of intra-government debt will be netted out. It will be defined for the purposes of the program as total outstanding gross debt liabilities. It will include, but not be limited to, liabilities in the form of securities and loans. It will exclude accounts payable. Debt will be measured at nominal value. The program exchange rates will apply to all non euro-denominated debt. Inflation indexation will apply to inflation indexed debt, using the relevant index as specified in the debt instrument. For the purposes of the program, the ceiling on the stock of central government debt will exclude debt arising from payments for bank restructuring, when carried out under the program's banking sector restructuring strategy (this does not cover the debt related to the Financial Stability Fund). This includes loans to financial institutions and investments in equity of financial institutions (requited recapitalization); unrequited recapitalization; and purchase of troubled assets. However, any financial operation by the central government to support banks, including the issuance of guarantees or provision of liquidity, with the exception of Hellenic Republic intermediation in repos between foreign and domestic financial institutions will be immediately reported to IMF, European Commission and ECB staff.

13. Adjusters. The ceiling on the overall stock of ESA95 central government debt will be adjusted upward (downward) by the amount of any upward (downward) revision to the stock of end-September 2012 ESA95 central government debt of $€ 307.9$ billion.

14. Supporting material. Data on the total stock of central government debt will be provided to the European Commission, ECB and IMF staff by the General Accounting Office consistent with the ESA95 definition no later than 30 days after the end of each month.

\section{F. Ceiling on New Central Government Guarantees (Performance Criterion)}

15. Definition. The ceiling on the new central government guarantees shall include new guarantees granted by the state, as well as new guarantees granted by any other entity that is classified under ESA95 under central government, but exclude guarantees to entities whose debt is covered under the ceiling on the stock of central government debt as defined in paragraph 15. The ceiling shall exclude: (i) guarantees to support banks; (ii) guarantees related to EIB financed loans; (iii) guarantees granted by ETEAN (up to an amount of $€ 50$ million provided these are fully backed by an equivalent amount of bank deposits); and (iv) guarantees granted under a risk sharing instrument of the EU structural funds (see COM (2011) 655 final) that do not create contingent liabilities for the Greek State. New guarantees are guarantees extended during the current fiscal year, but for those for which the maturity is being extended beyond the initial contractual provisions, only 50 percent of the full value will be counted. Modification of existing 
guarantees (without changing the maturity, amount of guarantees, and beneficiaries of the loan) will not be treated as new guarantees.

16. Supporting material. All new central government guarantees will be reported in detail, identifying amounts and beneficiaries. The General Accounting Office will provide the data on a monthly basis within three weeks after the end of each month. Non-state entities classified under the central government shall report the new guarantees they extended to the General Accounting Office on a monthly basis within three weeks after the end of each month.

\section{G. Non-Accumulation of External Debt Payment Arrears by the General Government (Continuous Performance Criterion)}

17. Definition. For the purposes of the program, an external debt payment arrear will be defined as a payment on debt to non-residents contracted or guaranteed by the general government, which has not been made within seven days after falling due. For purposes of this program, the term "falling due" means the date in which external debt payments are due according to the relevant contractual agreement, including any contractual grace periods. The performance criterion will apply on a continuous basis throughout the program period.

18. Supporting material. The stock of external arrears of the general government will be provided by the General Accounting Office with a lag of not more than seven days.

\section{H. Floor on Privatization Proceeds (Indicative Target and Performance Criterion)}

19. Definition. Privatization proceeds will be defined as the cash receipts from the asset sales to be carried out by the privatization agency (HRADF), cash receipts from direct government sales, and cash receipts from the eventual sale of any bank participations through the HFSF, the HRADF, or from the government directly. These will include, but not be limited to, the sale of equity of listed or non-listed companies and banks, shareholdings in public infrastructure, shareholdings in SPVs, leasehold in commercial real estate and publicly held land, sale-lease back operations, securitization of asset-related cash streams, or other assets incorporated in the authorities' privatization program, as well as sale of rights and concessions (including securitization of the proceeds of concessions). Proceeds will be valued in euro and reported on a gross basis, excluding any associated capital expenditure or other restructuring costs as well as the operating costs of the HRADF including the fees of advisors related to the specific privatization. Proceeds will be measured as the inflows of cash received by the HRADF as deposited in the Special Privatization Account at the Bank of Greece within 10 days after the settlement of the transaction.

20. Supporting material. Quarterly information on the cash receipts from asset sales, quarterly balances of the privatization account, inflows to the account (by project), and outflows to the state budget, will be made available by the Minister of Finance, in collaboration with the HRADF, no later than 60 days after the end of each quarter. 
21. Other. The privatization agency will provide $\mathrm{GAO}$, analytical data on the gross receipts and expenditures of the above mentioned sources, on a monthly basis-by the end of the $20^{\text {th }}$ of every next month.

\section{ESA “Program" Deficit and Overall Monitoring and Reporting Requirements}

22. ESA program deficit. For the purposes of the program, the ESA deficit (EDP B.9) will exclude the sale of non-financial assets such as land, buildings, and other concessions or licenses, unless these have been agreed in the context of the May 2010 SBA program (including subsequent reviews). The ESA deficit will also exclude all transfers related to the Eurogroup decisions of February 21, 2012 and November 26, 2012 in regard to income of euro zone national central banks, including the BoG, stemming from their investment portfolio holdings of Greek government bonds (schedule B provides the latest estimates).

Schedule B: Indicative Amounts to be Transferred to the Greek Government by Eurosystem National Central Banks (Billions of euros)

\begin{tabular}{lrrrrrrrrrr}
\hline & 2012 & 2013 & 2014 & 2015 & 2016 & 2017 & 2018 & 2019 & 2020 & Total 12-20 \\
\hline ANFA & 349.0 & 636.0 & 518.0 & 500.0 & 557.0 & 464.0 & 367.0 & 306.0 & 253.0 & $3,950.0$ \\
SMP & & $2,098.0$ & $1,941.0$ & $1,503.0$ & $1,134.0$ & 898.0 & 729.0 & 580.0 & 422.0 & $9,305.0$ \\
\hline
\end{tabular}

Source: Greek authorities, ECB, IMF staff estimates.

23. ESA primary balance. For the purpose of the program, the ESA primary balance is defined as general government ESA95 balance (EDP B.9) minus ESA 95 general government consolidated interest payable (EDP D.41).

24. Overall monitoring and reporting requirements. Performance under the program will be monitored from data supplied to the EC, ECB, and IMF by ELSTAT, the Ministry of Finance, the General Accounting Office, and Bank of Greece. The authorities will transmit to the IMF, EC, and ECB staff any data revisions in a timely manner.

\section{Monitoring of Structural Benchmarks}

25. Benchmark on progress in revenue administration, 2012-13. Progress in revenue administration in 2012 and 2013 will be defined as reaching or exceeding the targets set in Table 1.

26. Definition. A completed audit is defined as an audit reported as formally finalized in the ELENXIS audit case management system, including signed off by the audit supervisor, and the taxpayer has settled or appealed the assessment, or the audit report states that no underpayment has occurred. Risk-based audits for large taxpayers are defined as audits selected on a risk basis using the ELENXIS audit management system. The number of cases submitted to 
the state prosecutor relates to those submitted for financial crimes on grounds of suspicion of deliberate tax evasion. An audit of assets of a manager, director or auditor includes an audit of all assets, both movable and immovable, including those of his/her spouse. This will include but will not be limited to an examination of all his/her financial accounts for a period of up to 10 years from a current date, all immovable assets compared against information from State registries and of the acquisition of all moveable assets. The purpose of this audit will be to trace and justify the legal acquisition of these assets. These audits will be conducted annually by the Internal Affairs Directorate of the MoF.

27. Supporting material. Monthly information on risk-based full-scope audits and temporary audits of large taxpayers, self employed and high wealth individuals, and VAT nonfilers, collection of assessed taxes and penalties, collection of tax debt, and audits of asset declarations from auditors and managers of local tax offices will be made available by the Minister of Finance no later than two weeks after the end of each month. The monthly submission will also include, for each local tax office and special unit, the number of audits, hours spent on audits, assessed tax specified for income tax and VAT, assessed penalties and surcharges, collected tax amount from assessments, collected penalties and surcharges from assessments, specified for temporary and full scope audits.

28. Benchmark on progress in public financial management, 2012. Progress in implementing public financial management reforms in 2012 will be defined as reaching or exceeding the targets set in Table 2.

29. Definition. For the purpose of the 2012 target, the reporting institutional units (State and general government entities) include any unit under the general government as defined in paragraph 32 of the March 2012 TMU. For the purpose of subsequent target, the reporting institutional units (state and general government entities) include any unit under the general government as defined by ELSTAT as of end-September 2012 whose overall annual spending exceeded $€ 1$ million in 2011. From March 2013, entries under the e-portal will include all fields with financial information as prescribed in the GAO circular of Dec 29, 2010 (protocol number 2/91118/0026); this includes inter alia cumulative appropriations released, commitments made, the sum of invoices received, and payments made.

30. Supporting material. Monthly summary information from the e-portal, surveys, and other sources on performance against the above indicators will be published by the General Accounting Office of the Ministry of Finance on their website no later than four weeks after the end of each month. Data submission will include data back to end-2011. Survey information will continue to be provided after June 2013 unless discrepancies between survey and e-portal data are fully eliminated. An authoritative list of entities included under general government as defined by ELSTAT will be made available by the Ministry of Finance by December 31, 2012, updated with the entities reporting either on the e-portal or through surveys, including their share of overall annual spending in 2011 and the amount, provided no later than four weeks after the end of each month. 


\begin{tabular}{|c|c|c|c|}
\hline \multicolumn{4}{|c|}{$\begin{array}{l}\text { Table AIV.1. Greece: Structural Benchmarks in Revenue Administration, } \\
\qquad 2012-13\end{array}$} \\
\hline \multirow[t]{2}{*}{ Indicator } & \multirow{2}{*}{$\begin{array}{l}2012 \text { Target } 1 / \\
\text { End-Dec. }\end{array}$} & \multicolumn{2}{|c|}{2013 Targets 1/ } \\
\hline & & End-Jun. & End-Dec. \\
\hline \multicolumn{4}{|l|}{ Debt collection } \\
\hline Collection of tax debts as of the end of the previous year & 2,000 & 775 & 1,900 \\
\hline $\begin{array}{l}\text { Collection of new debts in the current year (percent of new } \\
\text { debt in the year) }\end{array}$ & $20.0 \%$ & $14.0 \%$ & $24.5 \%$ \\
\hline \multicolumn{4}{|l|}{ Tax audits and collection of large tax payers } \\
\hline Number of risk-based full scope audits in the year & 300 & 330 & 750 \\
\hline $\begin{array}{l}\text { Number of risk based temporary audits in the year } \\
\text { of which field audits }\end{array}$ & $\begin{array}{l}325 \\
100\end{array}$ & 350 & 750 \\
\hline $\begin{array}{l}\text { Collection full scope audits in the year (percent of assessed } \\
\text { tax and penalties) }\end{array}$ & $50 \%$ & $65 \%$ & $75 \%$ \\
\hline $\begin{array}{l}\text { Collection temporary audits in the year (percent of assessed } \\
\text { tax and penalties) }\end{array}$ & $50 \%$ & $45 \%$ & $55 \%$ \\
\hline Number of cases submitted to the state prosecutor & & 8 & 18 \\
\hline \multicolumn{4}{|l|}{ Audits and collection of high wealth individuals } \\
\hline Number of completed risk-based audits in the year & 1300 & 1200 & 2600 \\
\hline $\begin{array}{l}\text { Collection of assessed audits in the year (percent of assessed } \\
\text { tax and penalties) }\end{array}$ & $50 \%$ & $40 \%$ & $65 \%$ \\
\hline Number of cases submitted to the state prosecutor & & 50 & 110 \\
\hline \multicolumn{4}{|l|}{ Audits and collection for VAT non filers } \\
\hline $\begin{array}{l}\text { VAT audits of VAT non-filers } \\
\text { Collection of assessed tax and penalties }\end{array}$ & $\begin{array}{c}10,000 \\
20 \%\end{array}$ & & \\
\hline \multicolumn{4}{|l|}{ Internal control and human resource integrity } \\
\hline MoF audit of assets of managers of local tax offices $2 /$ & & 50 & 110 \\
\hline MoF audit of assets of auditors $2 /$ & & 50 & 130 \\
\hline \multicolumn{4}{|l|}{$\begin{array}{l}\text { 1/ Cumulative audits from January of each year. } \\
\text { 2/ The audit is performed by the Internal Affairs Directorate of the MoF. }\end{array}$} \\
\hline
\end{tabular}


Table AIV.2. Greece: Structural Benchmarks in Public Financial Management, 2012-13

Indicator
2012 Target

End-Dec.

\author{
2013 Target 2/
}

End-Jun. End-Dec.

a. Percent of institutional units (State and general government entities) reporting on the E-portal of GAO total budget allocations (including any revisions), pending outstanding commitments, unpaid commitments, and arrears data (for both ordinary and investment) at the end of each month, based on data from their commitment registers, is above the target.
2012 entity coverage
$90 \%$
2013 entity coverage
$80 \%$
$97 \%$

b. Discrepancy between the total arrears to third parties of non-state general government entities reported under the E-Portal of GAO using data from commitment registers and the total arrears reported through monthly surveys, i.e. the sum across all entities of the absolute value of (arrears monthly survey less arrears Eportal) divided by total arrears multiplied by 100 , is below target.
2012 entity coverage
$1 \%$
2013 entity coverage
$10 \%$
$1 \%$

c. Percentage of institutional units (State and general government entities) reporting on the E-portal of GAO all the prescribed items with financial information of the circular on commitment registers at the end of each month, based on data from their commitment registers, is above the target.

$$
2013 \text { entity coverage } \quad 65 \% \quad 93 \%
$$

$1 /$ ror the purpose of the 2012 target, the reportıng instıtutıonal units (State and general government entities) include any unit as was defined in paragraph 32 of March 2012 TMU. For the purpose of the 2013 target, the reporting institutional units (state and general government entities) include any unit under the general government as defined by ELSTAT as of end-September 2012, whose overall annual spending exceeded $€ 1$ million in 2011.

2/ Cumulative target from January 2013, reflecting the expansion of general government entities in the Elstat register as of September 30, 2012. 


\section{APPENDIX VI. GREECE-LETTER OF INTENT TO THE EUROPEAN COMMISSION AND THE EUROPEAN CENTRAL BANK}

Athens, 7 December 2012

Mr. Jean-Claude Juncker,

President,

Eurogroup,

Brussels.

Mr. Olli Rehn,

Vice President for Economic and Monetary Affairs and the Euro,

European Commission,

Brussels.

Mr. Mario Draghi,

President,

European Central Bank,

Frankfurt am Main.

Dear Messrs Juncker, Rehn and Draghi,

The attached Memorandum of Economic and Financial Policies (MEFP) and Memorandum of Understanding on Economic Policy Conditionality (MoU) outline the economic and financial policies that Greece will implement during the remainder of 2012 and in the period 2013-16. These policies aim to address Greece's competitiveness gap, support growth and employment, restore public finances to sustainability, secure financial system stability and distribute the cost of adjustment in an equitable way, within our commitment to the common currency.

The needed adjustment of the Greek economy has continued and the primary deficit has declined considerably. Following far-reaching labour market and other structural reforms taken in early 2012 under the programme, Greece's competitiveness gap and current account deficit are shrinking perceptibly. However, the economic contraction has been deeper-than-expected in 2012.

In support of our objectives, we have committed to a set of key policies, building on what has been achieved under the programme agreed in May 2010 and March 2012. These policies place strong emphasis on restoring growth and ensuring an equitable fiscal adjustment:

- The government has adopted an ambitious fiscal programme for 2013-16. We have already adopted: (i) a 2013 budget and a revised medium-term fiscal programme; and (ii) an implementation bill containing the fiscal measures for 2013-14. Our revised fiscal programme targets the $4 \frac{1}{2}$ percent of GDP primary surplus target by 2016, two years 
later than foreseen previously. The smoother path will help to moderate the impact of fiscal adjustment on the economy.

- We have also agreed to a number of safeguards for the delivery of fiscal commitments. These include stronger budgetary and monitoring requirements, with a wider role for the Ministry of Finance, automatic expenditure cuts when the fiscal performance deviates from established targets, fiscal rules for the general government, notably for line Ministries, state owned enterprises and local governments among others, a corrective mechanism on the primary surplus if privatisation proceeds fall short of targets, and changes in the governance of privatisation if the sale of assets is not in line with plans. A revenue rule for the general government has been agreed, according to which at least $30 \%$ of windfall revenues will be devoted to debt repayment while up to $70 \%$ could be used the following year by the Government to support temporary policies aiming to boost growth and social cohesion automatically, conditional to the achievement of the fiscal targets.

- Strengthening fiscal institutions is a crucial part of our reform programme. The government has already strengthened the capacity of the Tax Revenue Administration, simplified tax accounting procedures, and taken measures to secure tighter control over general government spending. These measures will help improve payment of taxes and prevent future accumulation of arrears. Further reforms in this area are planned in order to boost revenue collection, which is crucial for achieving equitable fiscal consolidation. Progress in this area is of paramount importance.

- To restore competitiveness and growth, our policies aim to accelerate the implementation of deep structural reforms in the labour, product and service markets. We have already taken a number of steps to help liberalize key product and service markets, restarted the privatization process, and reduced non-wage labor costs. These reforms will strengthen competitiveness, help catalyze much-needed investment, and protect real household income. Further reforms are planned throughout 2013-16 which will bring about long-lasting changes in the economy and will boost growth.

- We are strengthening the banking system, to further underpin the recovery. We have finalized the banking sector recapitalization framework, and introduced reforms to strengthen governance. These actions should strengthen depositor confidence and support renewed bank lending. We will proceed with the recapitalization of banks, in order to enable them to start lending again. This, together with the clearance of arrears in accordance with the framework agreed with the European Commission, the IMF and the $E C B$, is much needed for growth over the medium term.

We believe that the policies set forth in the attached memoranda are adequate to achieve the objectives under the programme and stand ready to take any measures that may become appropriate for this purpose as circumstances change. We will consult with the European Commission, the IMF and the ECB before the adoption of any such actions and in advance of revisions to the policies contained in these memoranda. 
The implementation of our program will be monitored through quantitative performance criteria and structural benchmarks as described in the attached MEFP, MoU and Technical Memorandum of Understanding (TMU). The quarterly reviews will assess progress in implementing the program and reach understandings on any additional measures that may be needed to achieve its objectives.

On this basis, we request the disbursement of the amount of EUR 43.7 billion, as agreed in the Eurogroup of 27 November 2012, stemming out of the financing arrangements by the EFSF supporting the Second Adjustment Program for Greece.

This letter is being copied to Ms Lagarde.

$$
\text { /s/ }
$$

\section{Antonis Samaras}

Prime Minister

/s/

Yannis Stournaras

Minister of Finance /s/

George Provopoulos

Governor of the Bank of Greece 


\section{APPENDIX VII. GREECE-MEMORANDUM OF UNDERSTANDING ON SPECIFIC ECONOMIC POLICY CONDITIONALITY}

The disbursements of financial assistance to Greece, by the European Financial Stability Facility (EFSF), are subject to quarterly reviews of conditionality for the duration of the arrangement. The release of the tranches is based on observance of quantitative performance criteria and a positive evaluation of progress made with respect to policy criteria in Council Decision 2011/734/EU of 12 July 2011 (as amended; hereinafter the Council Decision), the memorandum of economic and financial policies (MEFP) and in this Memorandum of Understanding.

The annex on data provision is part of the Memorandum and how well it has been respected will be considered in the assessment of compliance.

Greece commits to consult with the European Commission, the ECB and the IMF staff on the adoption of policies falling within the scope of this Memorandum allowing sufficient time for review. The Government publishes a quarterly report in line with Article 4 of the Council Decision.

In line with the conclusions of the euro-area summit of 26 October 2011, the Government will fully cooperate with the Commission, the ECB and the IMF staff teams to strengthen the monitoring of programme implementation, and will provide the staff teams with access to all relevant data and other information in the Greek administration.

The ownership of the programme and all executive responsibilities in the programme implementation remain with the Greek Government. 
2 STRUCTURAL REFORMS WITH BUDGETARY RELEVANCE

2.1 Privatising to boost efficiency in the economy and reduce public debt ...............................171

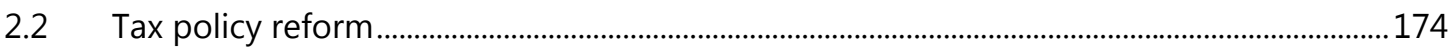

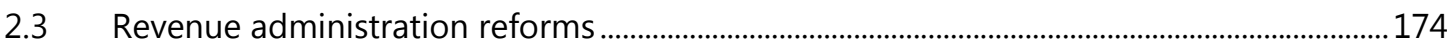

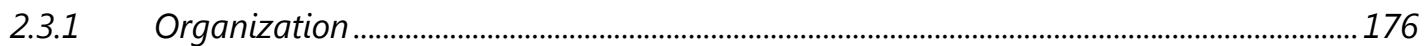

2.3.2 Fight against tax evasion, money laundering and corruption .........................................176

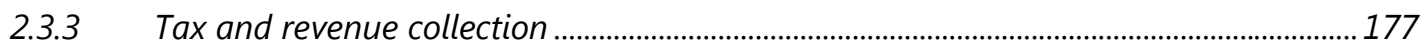

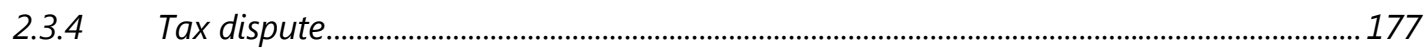

2.3.5 Management of the State Revenue Service ..................................................................... 177

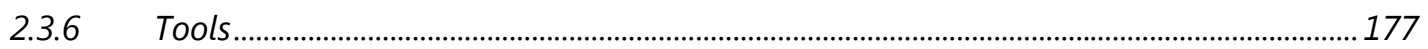

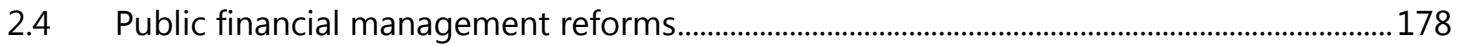

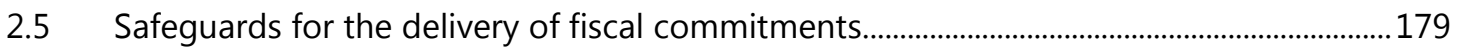

2.5.1 Enhancing national budgetary rules in line with the EU's Fiscal Compact................... 181

2.5.2 Budget preparation and implementation ......................................................................... 181

2.5.3 Monitoring and reporting ................................................................................................ 181

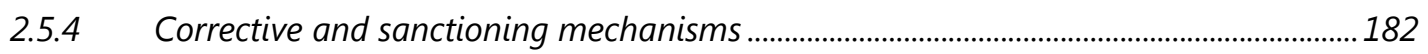

2.5.5 Transparency, accountability and oversight ...................................................................... 182

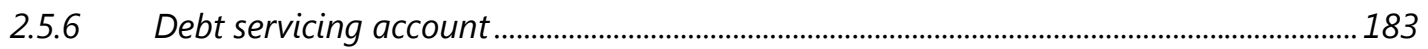

2.6 Other institutional requirements ................................................................................................... 183

2.7 Making the public administration more efficient and effective ............................................. 184

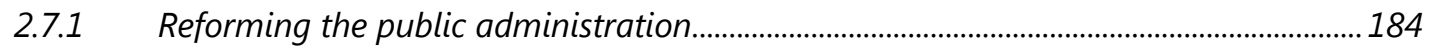

2.7.2 Avoiding waste and increasing quality through sound public procurement................. 186

2.8 Completing the pension reform to secure sustainability........................................................... 189

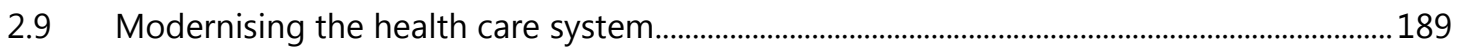

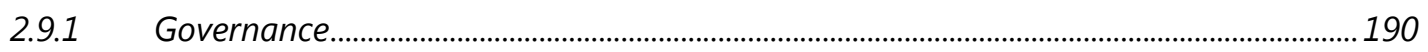

2.9.2 Controlling pharmaceutical spending ................................................................................. 190

2.9.3 Reviewing the provision of medical services contracted by EOPYY ................................ 194

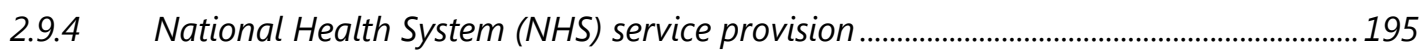

2.9.5 Centralised procurement ...................................................................................................... 197

2.10 Upgrading the education system................................................................................................ 197

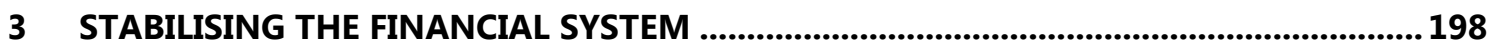

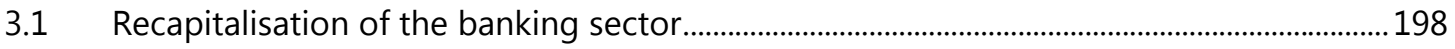

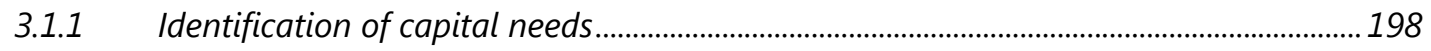

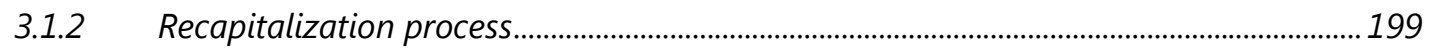

3.2 Framework for restructuring and strengthening of the banking system .............................200

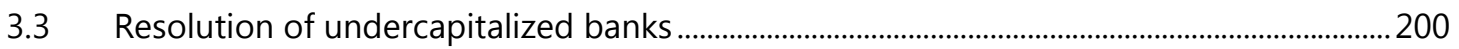

3.4 Safeguards to ensure stability and viability of the financial system......................................201

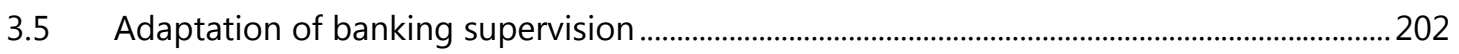

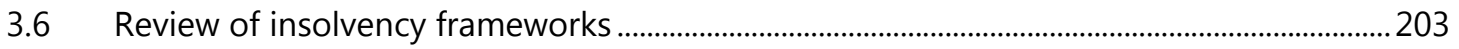

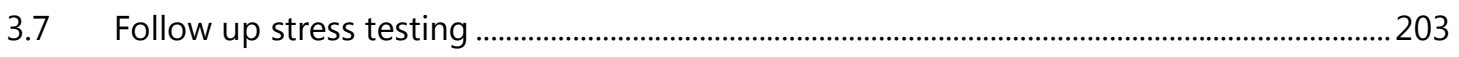

4 STRENGTHENING LABOUR MARKET INSTITUTIONS AND PROMOTING EMPLOYMENT ... 204 


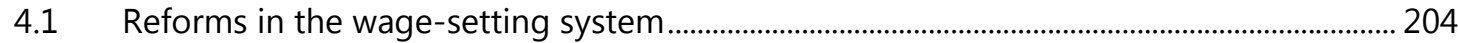

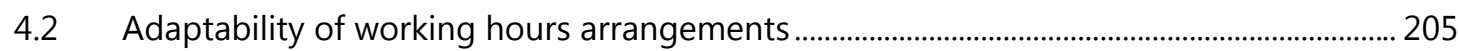

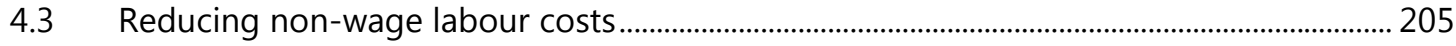

4.4 Lowering compliance costs, fighting undeclared work and informality ................................. 206

4.5 More transparent and enforceable labour law............................................................................. 206

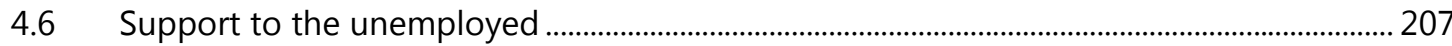

5 CREATING FAVOURABLE CONDITIONS FOR ECONOMIC ACTIVITY ................................207

5.1 Promoting an efficient and competitive business environment................................................ 207

5.1.1 Rationalising / eliminating quasi-fiscal charges ................................................................. 207

5.1.2 Reducing procedural and other administrative burden ..................................................... 208

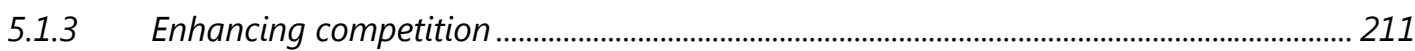

5.2 Reforming the judicial system to support economic activity................................................... 212

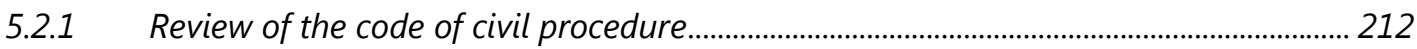

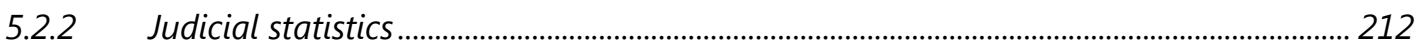

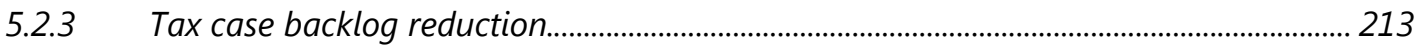

5.2.4 Non-tax case backlog reduction …………………............................................................... 213

5.2.5 Reorganization of the magistrates' court .......................................................................... 213

5.2.6 Development of e-justice applications in courts ................................................................ 213

5.2.7 Promotion of pre-trial conciliation and mediation .............................................................. 214

5.2.8 Other measures on judicial reform ................................................................................... 214

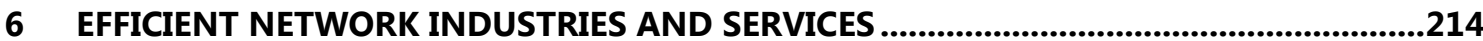

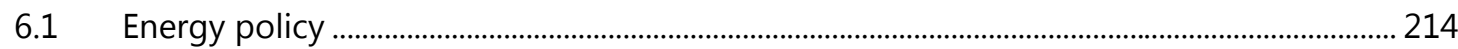

6.1.1 Unbundling effectively network activities from supply activities .................................... 215

6.1.2 Provisions regarding the privatisation of PPC and DESFA: ...........................................2. 215

6.1.3 Ensuring that electricity prices reflect costs ...................................................................... 215

6.1.4 Implementing smart metering systems............................................................................. 216

6.1.5 Providing for a financially sustainable development of renewable energy sources.. 216

6.1.6 Planning the development of the electricity market in the medium to long term..... 217

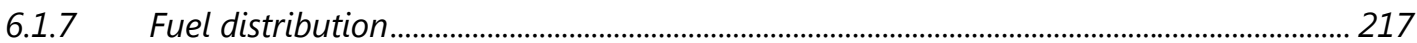

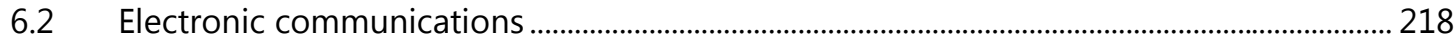

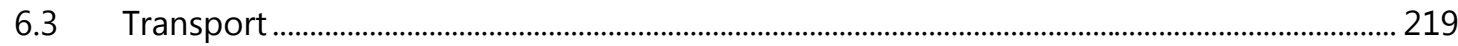

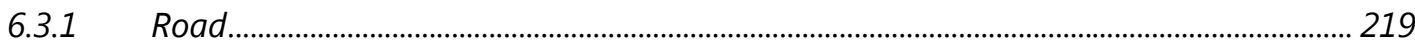

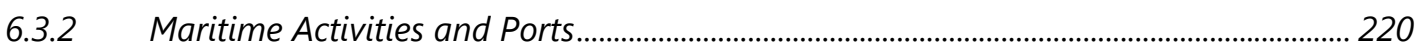

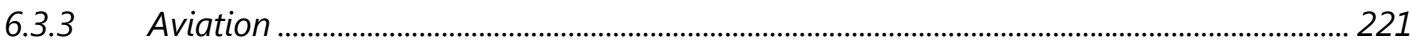

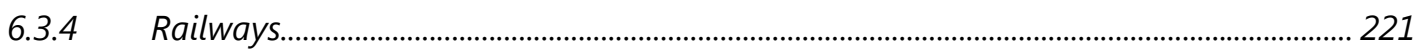

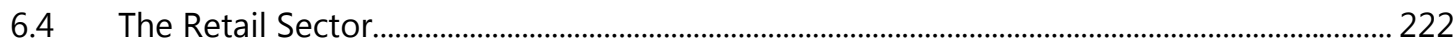

6.5 Regulated professions, professional qualifications and provision of services ................... 223

6.5.1 Removing restrictions to the access to and exercise of regulated professions.............. 223

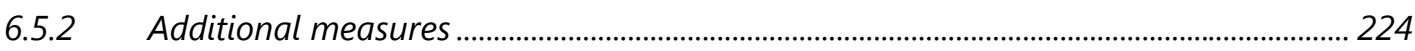

6.5.3 Easing the recognition of professional qualifications.......................................................... 225

6.5.4 Services Directive: exploiting the information benefits of the Point of Single Contact .....226

7 INCREASING THE IMPACT OF STRUCTURAL AND COHESION FUNDS 
8 MONITORING

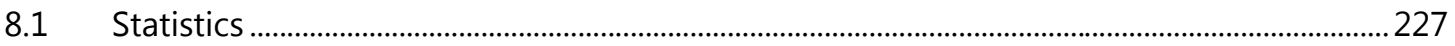

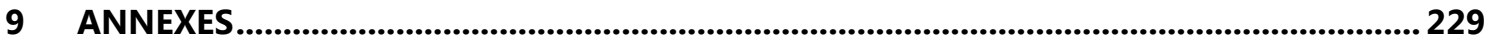

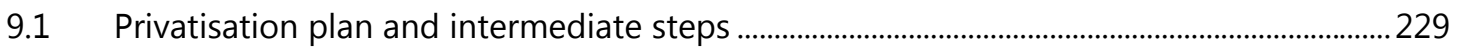

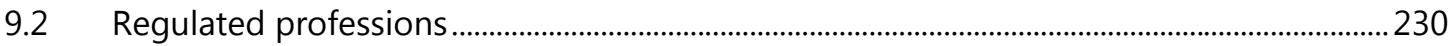

9.2.1 List no. 1: list of restrictions on selected regulated professions to be repealed prior to

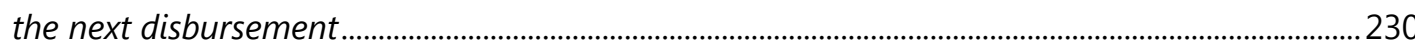

9.2.2 List no. 2: regulated professions / economic activities whose regulatory framework needs to be adjusted to applicable opinions of the Hellenic Competition Commission ...............232

9.3 Agreed roadmap between the Greek Ministry of Justice and the EC/IMF/ECB for the

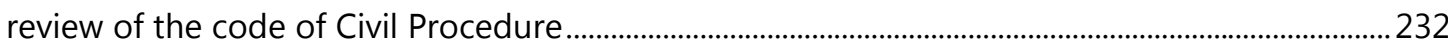

9.4 Statistics to be published by the Ministry of Justice or Ministry of Finance ........................233

9.5 Non-exhaustive list of regulations on port work for review under the new port strategy ....235

9.6 Additional fiscal measures for 2012 and Medium-Term Fiscal Strategy 2013-16 ............235

9.7 Provision of data ......................................................................................................................2. 


\section{Achieving sound public finances}

The fiscal adjustment path has been extended by two years to take into account the deeperthan-expected economic recession.

The two-year extension of the adjustment period will mitigate the impact on the economy, while securing a sustainable fiscal position. Under the revised adjustment path the primary balance targets have been set at $0 \%, 1.5 \%, 3 \%$ and $4.5 \%$ of GDP for the four-year period 2013-2016, respectively.

The extension of the adjustment period should not be seen as a way to reduce the effort, thereby weakening the credibility of the programme. To the contrary, the fiscal efforts needed to achieve the target remain very large in $2013-14$, and heavily frontloaded. Although the primary balance is only expected to improve by $1.5 \%$ of GDP per year, the cyclical adjusted primary balance (CAPB) is expected to increase by at least $2 \%$ of GDP in 2013, as the economic recovery is not expected to materialise until the end of 2013. Hence, even if the improvement in the nominal primary balance target is less ambitious than in March, the deeper recession means that the adjustment effort is equally strong.

The measures needed to reach the revised primary balance targets in the central macroeconomic scenario amount to EUR 9.2 billion and EUR 13.4 billion in 2013-14, respectively. The revised path for the primary balance means that the general government budget balance will fall below $3 \%$ of GDP in 2016, two years later than originally envisaged.

\section{Fiscal consolidation}

The adjustment path towards the correction of the excessive deficit shall aim to achieve a general government primary deficit (deficit excluding interest expenditure) not exceeding EUR 2,925 million (1.5\% of GDP) in 2012, and general government primary surpluses of at least EUR 0 million ( $0.0 \%$ of GDP) in 2013, EUR 2,775 million (1.5\% of GDP) in 2014, EUR 5,700 million (3.0\% of GDP) in 2015 and EUR 9,000 million (4.5\% of GDP) in 2016. Prior to any debt-reducing measures agreed in December 2012, these targets for the primary deficit/surplus imply an overall ESA-government deficit of $6.9 \%$ of GDP in $2012,5.4 \%$ of GDP in $2013,4.5 \%$ of GDP in $2014,3.4 \%$ of GDP in 2015 and $2.0 \%$ of GDP in 2016.

These numbers could be estimated to translate into an improvement in the cyclically-adjusted primary balance to GDP ratio from $4.1 \%$ in 2012 to $6.2 \%$ in 2013 and at least $6.4 \%$ of GDP in 2014,2015 and 2016 and into a cyclically-adjusted government deficit to GDP ratio at $-1.3 \%$ in $2012,0.7 \%$ in $2013,0.4 \%$ in $2014,0.0 \%$ in 2015 and $-0.4 \%$ in 2016 , reflecting the profile of interest payments.

Proceeds from the privatisation of financial and non-financial assets, transactions related to bank recapitalisation, as well as all transfers related to the Eurogroup decision of 21 February 2012 in regard to income of euro zone national central banks, including the Bank of Greece (BoG), stemming from their investment portfolio holdings of Greek government bonds shall not reduce the required fiscal consolidation effort and shall not be counted in the assessment of these targets. 
The adjustment path referred to in the previous paragraph, taking into account the impact of the debt-reducing measures to be implemented in December 2012, would be consistent with a general government consolidated debt ratio to GDP of below 160\% in 2016.

\section{Prior to the disbursement the Government:}

a. Adopts the budget for 2013 .

b. Adopts additional measures (see Annex 9.6) with the aim of reducing the primary deficit to EUR 2925 million (1.5\% of GDP) in 2012, EUR 0 million (0\% of GDP) in 2013, and ensure a primary surplus of 2775 million (1.5\% of GDP) in 2014, 5700 million (3.0\% of GDP) in 2015 and 9000 million (4.5\% of GDP) in 2016.

c. Adopts the medium-term fiscal strategy (hereinafter MTFS) through 2016 and the respective implementing bill. The MTFS elaborates on the permanent fiscal consolidation measures, which ensure that the deficit ceilings for 2013-16 as established by the Council Decision are not exceeded, and that the debt-to-GDP ratio is put on a sustainable downward path.

1. Following a decision on the final details of the planned direct income tax reforms, the authorities will review the fiscal outlook for 2014 and agree with the EC/ECB/IMF on measures needed to close any residual gap for 2014, in the context of the next review of the program.

2. To the extent that a fiscal gap in $2015-16$ remains, the authorities could pursue several strategies to close this, including improving revenue by broadening the tax base through further reduction in exemptions and deductions; extending measures that are expiring; and targeted cuts in current expenditure. There are also opportunities to refocus the investment program for more effective support to growth. The authorities will specify concrete plans for 2015 no later than end-August 2013, when they will formulate a detailed fiscal program for 2014-2015 consistent with a primary surplus of $3 \%$ by 2015.

\section{Structural reforms with budgetary relevance}

\subsection{Privatising to boost efficiency in the economy and reduce public debt}

The privatisation of public companies contributes to the reduction of public debt, as well as to the reduction of subsidies, other transfers or state guarantees to state-owned enterprises. It also aims at increasing the efficiency of companies and, by extension, the competitiveness of the economy as a whole, while attracting foreign direct investment. This is why the Greek authorities have committed to proceed swiftly and efficiently with the Privatisation Plan, with the aim of collecting EUR 50 billion, even if the sale of assets goes beyond the duration of the Economic Adjustment Programme. Within this context, the Government is committed to insulate the privatisation process from political pressures. 
The provision of basic public goods and services by privatized industries will be fully safeguarded, in line with the national policy goals and in compliance with the EU Treaty and appropriate secondary legislation rules.

Prior to disbursement, the Government:

\section{Strengthens the institutional framework for privatisation by:}

a. Presenting an updated Privatisation Plan to Parliament with the 2012-2016 MTFS.

b. Publishing a semi-annual update of the Asset Development Plan, which will include a Portfolio Overview with a description of the privatisation assets, a timeline of planned tenders and targeted total receipts for the current and next year.

c. Amending the Articles of Association of the HRADF (Article 16.3.) in order to stipulate that the "due cause" required for substituting members of the Board of Directors is defined in particular by the undue suspension or by the intentional compromising of the objectives of the HRADF with acts or omissions of its Board members.

d. Amending Law 3986/2011 to require the publication of quarterly reports of the HRADF on activities and financial accounts, including a detailed profit and loss statement, a cash flow statement, and a balance sheet, within 60 days of the end of each quarter.

\section{Transfers ownership of assets to the HRADF by:}

e. Transferring to the portfolio of privatisation assets of the HRADF the full and direct ownership (shares or concession rights) of: Egnatia Motorway and the regional ports of Elefsina, Lavrio, Igoumenitsa, Alexandropolis, Volos, Kavala, Corfu, Patras, Heraklion, and Rafina.

f. Signing the contract between HRADF and the Ministry of Finance for the use of the voting rights for ELVO.

g. Issuing an Inter-Ministerial Decision that secures that the proceeds of the sales of the Digital Dividends are transferred to the HRADF.

h. Having the line ministries and other relevant entities providing the General Secretariat for Public Property with full access to the inventory of all real estate assets owned by the State.

\section{Eliminates the legal obstacles for the sale of assets by:}

i. Amending/repealing statutory provisions of companies that diverge from private company law (PPC, OLP and OLTH port authorities, HELPE, EYATH and EYDAP, ports, etc.), regarding any restrictions on voting rights of private shareholders

j. Launching the process to obtain the zoning and land planning permits (ESCHADA), i.e., submit the environmental and zoning study for Afantou and Kassiopi. 


\section{Proceeds with the appointment of advisors by:}

k. Launching tenders for the appointment of advisors for EAS, ELVO, South Kavala Natural Gas, Trainose, being consistent with the existing procurement rules.

Other actions to be taken by the Government include the following:

1. Rapid adoption of necessary primary and secondary legislation and implementation decisions, in consistency with the required actions for a swift Privatisation Plan (see Annex 9.1).

2. The establishment of a regulatory framework for airports (January 2013), the State Lottery, ports (April 2013) and water companies (December 2012).

3. The submission of a pre-notification to the Commission services with respect to the definition of the universal postal services and the compensation of the Universal Service Provider (December 2012).

4. The launch for tender of Egnatia Motorways (March 2013).

5. Trainose is transferred to the HRADF (March 2013) and the tender for its sale is launched (June 2013).

6. Transfer of forty new real estate assets (identified as "real estate assets lots 2 and 3 " in the Privatisation Plan) to the HRADF (March 2013).

7. Full identification and description of all the remaining real estate assets in the pool of 3,150 assets that have been preselected and pre-valued by the HRADF (December 2013).

8. Transfer of full and direct ownership of 1000 commercially viable real estate assets to the HRADF (by end-2013). The transfer will be done in four phases, based on concrete interim targets of 250 real estate assets per quarter (starting in January 2013).

9. Ensuring that there will be no transfer or withholding of any real estate assets, without prior consultation and agreement with the HRADF and the EC/IMF/ECB, to entities other than the HRADF, including to municipalities and the recently established pension fund SPV or other dedicated legal entities, or until such time as the assets necessary to supply the privatisation plan have been secured (Continuous).

10. Amendment, upon privatisation, of all statutory provisions (including on labour relations) to fully align them with private sector law (Continuous).

11. The HRADF will continue to be tasked with selling assets as quickly and effectively as possible. In particular, there will be no further political review once an asset has been transferred to the HRADF. To ensure that the HRADF is accountable in this role we will enhance the transparency of its operations. To this end, the HRADF will publish quarterly reports on its steps to facilitate privatisations, financial accounts, including a 
profit and loss statement, a cash flow statement, and a balance sheet, no later than 60 days after the conclusion of every calendar quarter (Continuous).

12. Securing privatisation receipts which, cumulatively since June 2011 , should be at least EUR 1.6 billion by end-2012, EUR 4.2 billion by end-2013, EUR 6.5 billion by end2014, EUR 7.7 billion by end-2015, EUR 11.1 billion by end-2016.

\subsection{Tax policy reform}

1. The Government will prepare a tax reform that aims at simplifying the tax system, eliminating exemptions and preferential regimes, thus broadening bases, and allowing a gradual reduction in tax rates as revenue performance improves. This reform relates to the personal income tax and corporate income tax. The reform will be adopted in December 2012 to entry into force in 2013.

2. By November 2012, the Government will announce the full schedule of intermediate steps - including legislative actions as well as technical steps needed - until the new tax system becomes effective. These intermediate steps will include public consultation and appropriate review by the European Commission, ECB and IMF staff.

3. By March 2013, the Government makes fully operational a standard procedure for revision of legal values of real estate to better align them with market prices under the responsibility of the Directory of Capital Taxation.

\subsection{Revenue administration reforms}

A strong and focused reform programme to be undertaken in the coming months must address all the weaknesses in the existing system and support the fight against tax evasion and corruption. The Government will reform the current institutional framework in line with that in many other OECD and EU economies to ensure more autonomy for the tax administration department, especially for day-to-day operations, while leaving policy matters in the hands of the Government. The reform can be undertaken in a gradual way after assessing carefully the impact of each step undertaken:

- The re-organisation of tax offices must take place to increase the efficiency of audits and tax collection, by creating specialised units to deal with specific taxpayer groups (e.g., large tax-payer unit, high wealth individual unit, and large debtor unit), regrouping local offices into more efficient offices, and tackling potential corruption.

- Methods must be improved to focus audits on substantial issues in order to detect tax evasion and not on mere observance of formal rules. This implies the replacement of the Code of Books and Records by a more modern and substantially simpler set of rules for tax record keeping. A new single tax procedure code will be created. Additionally, compulsory auditing of all tax declaration should be abolished, and efforts concentrated on high yield audits, targeted in using risk assessment techniques. 
- Collection of taxes should be reinforced. At the local level, groups of specialized and dedicated staff will be put into place in larger DOYs. Besides, rules to write-off noncollectable debts in line with international best practice have to be introduced.

- The management will be improved, under the leadership of a new Secretary General with increased powers. Managers and auditors should be subject to performance targets and regular assessment. The Secretary General must have the capacity to replace nonperforming managers and auditors. Besides, the regular rotation of managers has to become a rule.

- Fighting tax evasion and corruption is a priority in this effort. Progress made so far did rely on useful but piecemeal measures. Fight against corruption, especially in the tax sector, must use new tools, making full use of the will of the people to fight it.

- The current administrative review process has to be replaced by a cost effective compulsory pre-settlement administrative procedure, in order to significantly reduce the number of unnecessary tax litigation, so as to lighten the burden of courts and ensure a timely settlement of the cases.

With the aim of strengthening fiscal institution as part of the reform programme, the Government, prior to the disbursement:

a. Adopts legislation to define the role and qualifications of the Secretary General. Concerning qualifications, this will be a person with senior management experience, expertise in tax matters, and an impeccable reputation (including a strong tax compliance history).

b. Adopts interim legislation, and the Minister of Finance will use this to delegate decision making powers to the Secretary General. These powers will include the competence to make operational decisions, direct and control local offices, manage human resources, replace underperforming senior managers, manage the budget of the tax administration, and manage all information with due confidentiality;

c. Adopts legislation to deploy experienced tax auditors towards activities serving the immediate revenue imperative, making fully operational key enforcement areas as the large taxpayer unit by transferring 100 auditors, establishing one functional unit for high-wealth individuals and high-income self-employed and staffing the unit with 50 experienced tax auditors directly accountable to the Secretary General of the tax administration;

d. Establishes procedures for the rotation of managers in critical tax offices on a periodic basis.

e. Replaces the Code of Books and Records by significantly simpler legislation in line with international standards; 
The Government undertakes the following reforms:

\subsubsection{Organization}

1. Appoints a new Secretary General of the tax administration (December 2012);

2. Adopts legislation to establish a significantly more autonomous tax administration and specify the degree of autonomy, governance framework, accountability, legal powers of the head of the administration and initial staffing of the organization by February 2013. In March 2014 the new agency will become fully operational;

3. Continues to centralise and merge local tax offices leaving about 120 functioning offices (June 2013);

\subsubsection{Fight against tax evasion, money laundering and corruption}

1. The revised Code of Books and Records enters into force ( $1^{\text {st }}$ January 2013);

2. Steps up the hiring procedure and simplifies the reassessment process of the tax auditors with the objective to achieve the target of 2000 tax auditors fully operational by June 2013;

3. Introduces, at least, twice-yearly performance assessments for tax auditors (December 2012);

4. Issue an administrative circular to enhance targeted auditing based on risk assessment techniques (December 2012).

5. Abolish the requirement that all tax declarations for the previous 10 years must be audited while retaining the right to continue to audit earlier years and the discretion to audit any amount of declarations from these earlier years (January 2013).

6. Adopts legislation in order to introduce a modern code of conduct concerning conflicts of interests and declaration of interests and a system for protecting whistleblowers who report corruption (March 2013);

7. Appoints a national coordinator for anti-corruption action (April 2013);

8. Enacts the appropriate legal framework to create a secure direct or indirect central register of bank accounts (January 2013);

9. Requires that all Ministries which have a fiscal relationship with taxpayers utilize their tax identification number for financial transactions with them (June 2013).

10. Introduces a central agency to consolidate and link all of the different identification numbers now employed across various government agencies (June 2014); 


\subsubsection{Tax and revenue collection}

1. Establishes specialist debt management units in larger local tax offices and allocating at least 10 percent of local staff to this function (December 2012);

2. Completes a review of the policy and procedures to write off tax debts, and prepare recommendations to facilitate actively managing tax debt with real prospect of collection and explore ways to deal with the non-collectable part (February 2013);

3. Replaces payments in cash and cheque in tax offices with bank transfers, to discourage corruption and free up staff time for higher value added work (audit, collection enforcement and taxpayer advice) (December 2012);

4. Commits not to adopt new tax amnesties, or extend existing amnesties for the collection of taxes and social contributions during the years covered by the economic adjustment programme (Continuous);

5. Integrates the collection of social security contributions into the tax administration (March 2014).

\subsubsection{Tax dispute}

1. Puts in place a mandatory administrative review procedure (Q2 2013). The aim is to design a mandatory administrative appeal procedure in line with international best practice to allow a distinct and higher level body within the Ministry of Finance, staffed with specialists in tax dispute matters, to re-examine tax decision taken by the DOYs or auditors before going to court.

\subsubsection{Management of the State Revenue Service}

1. Replaces managers who do not meet performance targets (Continuous);

2. Launches an easily accessible website to enforce accountability to the public through publication of summary statistics on key performance indicators, the number of tax evasion cases sent to the FIU and to prosecution by the tax administration (December 2012).

\subsubsection{Tools}

1. Adopts a new Single Tax Procedures Code (June 2013). This code should aim to reduce the costs of administration and compliance and will incorporate procedural reforms in all major administrative areas that are necessary to support modern tax administration (e.g., tax filing, audit and penalties, enforcement powers and debt collection). It will also incorporate a new streamlined administrative dispute resolution process;

2. Puts in place a new IT system that interconnects all tax offices (March 2013); 
The preparation of the new IT system involves the following main steps in relation to the new data centre, web-facing and back-office applications:

3. 20 more new electronic services and enhancements (December 2012). These concern mainly taxes withheld at source;

4. 8 remaining new electronic services and enhancements (December 2012). These concern forms filed late with a fine, real-estate tax, and VAT administration;

5. system and user tests, user training, and migration of all tax offices to the centralized database (December 2012);

6. operational use of the new IT infrastructure by all tax offices (March 2013).

\subsection{Public financial management reforms}

Developing a solid public financial management framework is key in controlling expenditures and thus being able to achieve fiscal targets. The Government is committed to enact reforms for strengthening the framework both within the GAO and line ministries.

\section{Prior to the next disbursement the Government will,}

a. adopt the 2013-16 Medium term fiscal strategy

b. ensure that EOPYY reports for at least two consecutive months (retroactive reports included) from its commitment register through the e-portal.

\section{Further actions include:}

1. The Government adopts an administrative calendar for the update of the medium-term fiscal strategy. (February-2013)

2. To address problems still lying in the extra-budgetary funds and in the social security sector, especially in relation to the transfer of competencies from SSFs to EOPPY, despite the progress in the setting-up of the commitment/co-payment registries, the Government will:

i. ensure that by December-2012 commitment registers are in operation in 90 per cent of general government entities.

ii. monitor the effectiveness of the commitment registers by conducting regular targeted inspections in the public entities covered by the system (Continuous).

iii. enforce the obligation of accounting officers to report commitments by enacting sanctions to entities not submitting needed data, though disciplinary action for accounting officers, and by strengthening the role of 
GAO in providing support and guidance to Accounting Officers (Continuous)

iv. ensure by December 2012 that EOPYY monthly budget execution is published on the website with a lag of four weeks after the end of the respective month, providing detailed data on both expenditure commitments/purchases (accrual basis) and actual payments (cash basis), current performances against yearly budget allocation and accumulation of accounts payable (and arrears). As soon as significant deviations from yearly targets become evident, remedial action should be taken at the same time.

3. To clear expenditure arrears and tax refunds, the conditions for a government unit to meet to allow funds for clearance to be disbursed will include, for expenditure arrears: (i) establishment by the unit of a fully functioning commitment register and (ii) reporting of at least three months of consistent data on commitments, payments, and arrears (2 months for EOPYY); and, for both expenditure arrears and tax refunds: (iii) verification of claims. Subvented agencies which meet these conditions can clear their arrears even if their parent agency does not meet the conditions. Arrears should not delay the execution of the pharmaceutical spending clawback or any related measure. The Government will:

i. $\quad$ Prepare and publish by November 2012 a plan for the clearance of arrears owed to suppliers by public entities and of tax refunds (to be done by GAO in liaison with GSIS and other relevant authorities).

ii. Ensure the administrative capacity to make the clearance of arrears effective through different means, including re-allocation of at least 30 employees with relevant competences from other social security funds to EOPYY (November 2012)

4. Once the clearance of all verified arrears is achieved, the Government ensures that no new arrears are accumulated (Continuous).

5. The GSIS designs a risk-based assessment procedure for verification of VAT refunds (March 2013).

\subsection{Safeguards for the delivery of fiscal commitments}

Enhancing credibility is essential to the success of the Adjustment Programme for Greece. One way is through the early implementation of the EU's Fiscal Compact. Greece has already signed and ratified the intergovernmental Treaty on Stability, Coordination and Governance in the EMU. A key part of the Treaty is the fiscal compact that introduces national budgetary rules as well as enhanced enforcement mechanisms at European level. Within a comprehensive approach, key steps to safeguard the delivery of fiscal commitments are necessary in the areas of: Budget preparation and implementation, monitoring and reporting, corrective and sanctioning mechanisms, transparency, accountability and oversight, debt servicing. 


\section{The Government will, prior to disbursement:}

a. Adopt a Council of Ministers act (replacing the Council of Ministers act adopted on 29 October 2012, aiming at strengthening the Budget execution and enhancing the sound fiscal management), and including, beyond the provisions in the original Council of Ministers act, additional provisions:

i. establishing that Memoranda of Cooperation are signed by end-December of each year between the Ministry of Finance and the other Ministries or between the Ministries and managers of the supervised entities (thus covering the entire General Government). The Memoranda aim at enhancing the current monitoring and introducing corrective mechanisms, such as: quarterly budget execution targets, corrective actions in case of deviations from targets and further actions in case of non-compliance with the corrective actions.

ii. Strengthening the current balanced budget constraints for Local Governments in order to be more effective, but also including corrective and sanctioning mechanisms.

iii. Strengthening the current monitoring system for SOEs, introducing an enforcement mechanism in case of deviations from the specific targets identified for each SOE.

iv. Setting the framework for defining specific targets for the coverage of operational commitment registers for $L G$ and SOEs to be established by December of each year.

v. Setting up a framework for correcting transfers from central government to address deviations from targets within the year and possibly in the following years while ensuring that arrears are not increasing. Improvements in operational terms should be integrated in the relevant legislation, including inter alia triggering circumstances, criteria for graduation from suspension to outright reduction of transfers, and timing issues.

vi. Making it explicit that the proceeds from the privatisation of government assets are paid directly into the account referred to in section 2.5.6.

vii. Set automatic cuts in expenditures to be applied as a rule when targets are missed, while ensuring that arrears are not increasing.

viii. Reinforce centralisation of budget planning and implementation. This will include further strengthening of the Ministry of Finance vis-à-vis line ministries, notably with the introduction of an effective top-down budgeting, including a veto role of the Minister of Finance, monthly submission to the supervising Director General of Financial Services (DGFS) and the GAO (depending on the size of their budget) of the budget execution programme and actual execution, and the power to take corrective measures if appropriate at the implementation stage, with bodies 
failing to comply with their obligations being brought under the direct supervision of the Ministry of Finance.

1. The Council of Ministers act referred to in paragraph a. shall be converted into law by end-December 2012.

\subsubsection{Enhancing national budgetary rules in line with the EU's Fiscal Compact}

1. The Government will adopt the necessary legislation to transpose the Fiscal Compact provisions with a view to introducing a structural budget balance rule with an automatic correction mechanism (August 2013)

\subsubsection{Budget preparation and implementation}

The Government will:

1. Introduce three-years binding expenditure ceilings per subsectors (at least for the central government and the health sector) for 2013 , in order to strengthen the implementation of the MTFS, as follows: ceilings for the first two years would be considered fixed and used as such in the following budget planning exercise although with some flexibility within the ceiling as long as the general target is met to leave some space of action to the administration; ceilings for the last year of the three-year period may be updated annually. This measure will be adopted within an update of the MTFS to be done by January 2013.

2. Modify the organic budget law by August 2013 to introduce:

i. $\quad$ The three-years binding expenditure ceilings (as in paragraph 2.5.1) on a permanent basis as part of the rolling MTFS.

ii. Provisions to freeze ex-ante $10 \%$ of discretionary appropriations per budget line as part of the MTFS. The frozen appropriations would be released in the second half of the year conditional upon meeting the fiscal targets. The first application should concern the 2014 budget.

iii. A revenue rule for the general government, according to which at least $30 \%$ of windfall revenues will be devoted to debt repayment while up to $70 \%$ could be used the following year by the Government to support temporary policies aiming to boost growth and social cohesion automatically, conditional to the achievement of the fiscal targets.

\subsubsection{Monitoring and reporting}

The Government will:

1. Identify other areas of operational expenditure where real time monitoring mechanisms could be introduced or strengthened (March 2013). 
2. Extend e-prescription to illness benefits provided by EOPYY to strengthen the monitoring among others of Diagnostic Tests, Inpatient Care and Rest Provision. (June 2013)

\subsubsection{Corrective and sanctioning mechanisms}

The Government will:

1. Ensure a continuous balance between contributions and benefits, by bringing forward by one year the entry in force of the binding mechanism (for auxiliary pensions) already legislated to enter in force as of 2015. (September 2013)

2. Strengthen HRADF's governance and independence and implement an automatic correction mechanism, should there be any difficulties in the privatisation process or slippages in the targets, by (quarterly):

i. Reviewing the functioning of the recently amended privatisation law, through specific QPCs to be enforced the moment the privatisation plan derails.

ii. Taking, in cooperation with EC/ECB/IMF, appropriate steps, including changes in existing legislation and/or in the composition of the Board, to safeguard and strengthen the independence and the functioning of the HRADF, if targets for the sale of assets to be privatised were missed substantially for two consecutive quarters. In all circumstances, the HRADF remains fully accountable to parliament on an ex-post basis for the integrity of every privatisation sale.

iii. Increasing automatically the primary surplus target, should there be a shortfall of privatisation proceeds due to the delay in sales of specific assets compared to programme targets for two consecutive quarters. Any shortfall in privatisation proceeds ceteris paribus increases the financing need and the debt ratio. To mitigate this undesirable outcome, unless other adjustments are agreed with the $\mathrm{EC} / \mathrm{ECB} / \mathrm{IMF}$, the primary surplus target would be raised with immediate effect by 50 percent of the shortfall in proceeds, and should be achieved by means of current expenditure cuts in the general government. The adjustment within any year would be capped at $€ 1$ billion.

\subsubsection{Transparency, accountability and oversight}

The Government will:

1. Increase transparency and accountability to the public/parliament, by e.g. releasing status reports on the implementation of the legislated fiscal measures, publication of hiring numbers, proper fiscal impact assessment of legislation, statement of the main sources of fiscal risks related to changes in key economic assumptions in the forecast, as well as an assessment of the fiscal impact of the main sources of fiscal risk including government guarantees and other contingent liabilities, etc. (March 2013) 
2. Resume and enhance the operation of the existing Parliamentary Budget Office (June 2013) and take steps to strengthen its reputation, independence and technical competence towards a fully-fledged fiscal council (e.g. provision/endorsement of forecasts for the budget preparation, monitoring of compliance with budgetary targets and fiscal rules, provision of independent assessments of fiscal developments and challenges, etc.), building on best international practices. (December 2013)

\subsubsection{Debt servicing account}

1. The Government will ensure an effective implementation of the debt servicing account to monitor cash flows, avoid diversion of official financing and secure a timely debt servicing. Law 4063/2012 established a segregated account in the Bank of Greece. By law, disbursements to this account cannot be used for any other purposes than debt servicing. Via this account the amortization and interest payment costs of all HR's loans, debt management transactions and derivatives, as well as any parallel cost (fees and other expenses) related to debt servicing and in general to Public Debt Management are paid. The proceeds of this account are the disbursement of EFSF's loans, subject to an EFSF acceptance notice, as well as the Hellenic Republic's contributions to debt servicing, including all revenues from the privatisation of State assets and at least $30 \%$ of windfall revenues. All payments from this account will be subject to prior detailed reporting to the EFSF/ESM and ex-post confirmation by the account holder. (Continuous)

\subsection{Other institutional requirements}

\section{Prior to the disbursement, the Government:}

a. Adopts the legal act harmonising the entrance fees for all casinos in Greece and all necessary actions are taken toward full and effective recovery of the illegal state aid from all Casinos, including Casino Mont Parnes.

b. Identifies the assets and production units of LARCO assets and rights that belong to the Agios Ioannis/Larymna concession in view of their sale after the current concession of LARCO.

c. Amends the current requirement in the existing ETEAN law of providing government bonds at market value to banks when guarantees are called. Instead the State will provide ETEAN with cash against ETEAN holdings of government bonds to pay banks for the guarantees called.

\section{Other Actions}

1. The Government identifies the assets and production units of the Hellenic Defence Systems to be privatised. (December 2012)

2. The Government creates a Central State Aid Unit responsible for screening all measures, from across the Government, for their compliance with State aid rules, 
before they are implemented. (January 2013). The Central State Aid Unit will be the only contact point for the Commission on all State aid matters, including for notifications. The aim is to ensure a timely and effective clearance of state aid issues.

3. All actions attributable to public authorities should be in compliance with the rules on free movement of capital (TFEU, Article 63) (Continuous).

\subsection{Making the public administration more efficient and effective}

Reforming the public sector constitutes an essential step for the reduction in waste, the containment of public wages and the increase in efficiency and productivity levels. As a prerequisite, the following actions will be taken in the next months.

\subsubsection{Reforming the public administration}

In order to achieve a leaner and more efficient state, the Government initiates a rigorous evaluation of administrative structures and personnel, in order to maintain the right skill mix of employees over time. Entity closures are pursued and employees are either transferred to the mobility scheme or dismissed. This reform process is extended to extra budgetary funds and regional and local administrations in 2013.

Prior to disbursement:

a. The Government ensures that at least 2,000 staff will be moved to the mobility scheme.

\subsubsection{Institutional reforms}

1. The assessment of the institutional setting of two pilot ministries (Ministry of Administrative Reforms and Ministry of Environment) is completed (October 2012).

2. The assessment of the institutional setting of all ministries is finalised (December 2012), while the assessment of the performance of civil servants is completed (December 2013).

3. The first draft of the two pilot staffing plans is finalised (November 2012); the other ministries' staffing plans are completed (February 2013).

4. Develop an action plan for the assessment of all public entities, including all ExtraBudgetary Funds and SOEs under Chapter A (December 2012). The action plan will focus on their mandate and activities, their staffing, the level of State subsidies and their overall budget. The pilot assessment of two major public entities is completed by February 2013 and a complete assessment of all public entities is completed by December 2013.

5. Based on the different assessments, the Council of Reform approves the transformation scenarios for each ministry (January 2013). A precise roadmap on how these structures are created must be published two months after the approval of the transformation scenarios. 
6. The Government monitors progress on the inter-ministerial coordination, whereby the Coordinator is appointed (November 2012) and full implementation of the process is ensured (January 2013).

7. The Government involves the Commission services with respect to the assessment of structures and staffing linked with the implementation of the Cohesion Policy (NSRF Operation Programmes) and will seek its agreement when taking related decisions (Continuous).

8. Prepares and makes public a fully-fledged anti-corruption plan for the civil service, including special provisions for the tax and customs administration (February 2013).

\subsubsection{Mobility scheme and human resources management}

In order to ensure that the Government's efforts to redeploy and reduce personnel are effective, that the recruitment and evaluation process are improved, and that the performance and motivation of personnel increases, concrete steps will be made to: limit and improve the quality of hiring at the selection, limiting the intake into public service academies by 30 percent, removing job guarantees for private sector teachers; put a sunset to existing lists of eligible graduates; establish clear guidelines for the regular assessment of personnel run by managers.

The Government will:

1. Combine the assessment of the institutional setting with mobility, attrition, reduction of temporary contracts, disciplinary procedures and mandatory redundancies. This should help reduce the public sector workforce by 150,000 by 2015 , relative to the end-2010 level.

2. Place 25,000 government employees in the mobility scheme in 2013 , with half of them by mid-2013. The government's mobility scheme, where transferred personnel can remain for up to one year with a reduced rate of pay (substituting for severance payments), while they seek new employment and they are re-trained, will help the transition across job positions, if necessary towards the private sector.

3. By end-February 2013, the staffing plans for line Ministries will be completed and these will be used to identify redundant positions and employees, and on this basis set quarterly targets for mandatory exits through end-2014.

4. Adopt the law on mobility between ministries in the interest of the services (November 2012).

5. Defines a human resources strategy in order to: (i) identify the weaknesses of the public service management of human resources; (ii) identify the best possible way to modify recruitment procedures, appointments, trainings, as well as mobility. This strategy is reflected in legislation (January 2013). This legal act will provide a basis for evaluating the competences of the senior management. 
6. Assesses the mandate, roles and responsibilities of all senior managers, including the politically appointed and the senior public service management (February 2013). This assessment will lead to an amendment of the current legislation, by clarifying and framing the relationship between the political level, the management positions and the services. Within this framework, the number of advisors will be reduced and constrained, and each advisor will have a specific job description. The aim is to ensure institutional continuity and higher levels of efficiency in the public administration (May 2013).

\subsubsection{Avoiding waste and increasing quality through sound public procurement}

Important fiscal savings and higher quality purchases can be realised by sound public procurement processes. The reforms aim at i) making the Single Public Procurement Authority, the newly created procurement watchdog, fully operational; ii) establishing an e-procurement platform and mandating gradually its use by the public administration; iii) increasing the share of supplies and services tendered through Central Purchasing Bodies, including by the use of framework contracts and iv) at codifying and simplifying all public procurement legislation.

\subsubsection{To make the Single Public Procurement Authority (SPPA) operational}

The Government:

1. By December 2012, issues the following implementing legislation of Law 4013/2011 on the Single Public Procurement Authority, providing for:

i. the SPPA's financial management rules (Art. 4).

ii. the SPPA's structure and remit of its services and any other matters pertaining to its organisation (Art. 4).

iii. the SPPA's Rules of Procedure (Art. 7).

iv. the Agora Portal for contract transparency (Art. 11).

The above legislation shall enter into force at the latest by December 2012.

2. Ensures that all necessary staff is transferred to the SPPA, in accordance with the Presidential Decree 123/2-11-2012 on its organisation, so that the SPPA fulfils its mandate, objectives, competences and powers as defined in the implementing regulations, of the law on the SPPA and in the Action Plan agreed with the European Commission in November 2010. (February 2013)

3. The SPPA ensures coordination and coherence of the functioning of the Central Purchasing Bodies, of the reform of the Greek public procurement regulations and of the e-procurement framework with the overall public procurement system and strategy. (Continuous) 


\subsubsection{To increase the efficiency of procurement processes:}

The Government moves towards more centralised procurement, especially in the field of health procurement, services and supplies (including civil supplies and services for defence not falling under the scope of Directive 2009/81 on procurement in the fields of defence and security). It also uses framework contracts and reviews the public procurement legislation including works, supplies and services. In particular the Government:

Central Purchasing Bodies (CPB):

1. Presents a plan for the development of CPBs (such as the Greek General Secretariat for Commerce -for supplies and services- and EPY -for health procurement-) to the Commission Services by December 2012. The plan identifies all contracting authorities procuring supplies and services at the Central Government level and overviews their procurement needs, with a view to gradually increasing the share of supplies and services procured through those CPBs. Similarly, a plan to establish CPB at regional/local level is also submitted to the Commission services by December 2012.

Framework contracts:

2. Submits by December $\mathbf{2 0 1 2}$ to the Commission services for evaluation two and one framework contracts used in frequently purchased supplies or services at central government and regional level, respectively, and mandates the relevant administration to source via those contracts. (April 2013)

Reform of public procurement legislation:

3. Undertakes to adopt by December 2013 a reform of the public procurement system including works, supplies and services under the coordination of the SPPA with a view to:

i. simplifying, streamlining and consolidating the body of public procurement legislation;

ii. rationalising the administrative structures and processes in public procurement to desired procurement results in terms of efficiency and efficacy;

iii. improving national review procedures, including the reduction of delays triggered by the redress system and assessing the role to confer to the SPPA in the area of redress (remedies and judicial protection).

4. Develops an Action Plan for the reform, in agreement with the European Commission. (January 2013). It will include an analysis of the state of play (flowcharts, procedural phases, actors involved, timelines, statistics etc.) and factors in the results of the study on the Greek public procurement system commissioned by the EC. 
5. The drafts of all legislative and organisational measures implementing the abovementioned Action Plan are presented to the European Commission in September 2013.

\subsubsection{To run public procurement procedures by electronic means (i.e., E-procurement):}

The Government

1. Refines, in consultation with the European Commission, the existing plan for the development of the e-procurement platform by December 2012, including, among others, measures and deadlines for:

i. the operation of supplies, services and public works procurement contracts through the e-procurement platform;

ii. the availably of functionalities such as e-notification and e-tendering;

iii. the mandatory use of the platform by the central government, regional government and other public sector entities;

iv. the communication and training programmes for users of the platform;

v. the periodic monitoring mechanisms for the take-up of e-procurement platform by its users and the specification of target usage levels;

vi. the interaction of the platform with the planned simplification of procurement legislation;

vii. the means to facilitate access and use to the platform by users, including easy to use e-signature and e-ID solutions.

2. In the development of the e-procurement platform, commits to:

i. complete the e-procurement infrastructure for supplies and services contracts by December 2012.

ii. run supplies and services contracts for the Central Government on a pilot basis through the e-procurement platform throughout the $\mathbf{1}^{\text {st }}$ half of 2013.

iii. ensure that the e-procurement platform is fully operational and ready for use by the Central Public Administration for supplies and services contracts in July-2013.

3. Ensures the use of the platform as follows:

i. the Central Government procures at least $25 \%$ of its supplies and services' needs (in terms of contract value) through the e-procurement platform by December 2013. 
ii. the Central Purchasing Bodies (CPBs) use the e-procurement platform for all their tendering procedures. (June 2014)

iii. the whole public sector in Greece uses the e-procurement platform by December 2015.

4. Submits to the Commission services the data of the monitoring activities covering year 2013 against the target user levels. (1st half of January 2014)

\subsection{Completing the pension reform to secure sustainability}

\section{Prior to disbursement}

a. The age of retirement is increased by 2 years, starting from (1 January 2013). The increase is applied to the statutory retirement age and any other retirement age for special groups and to the minimum requirement for getting a pension.

\section{Other actions}

The Government:

1. Finalises the implementation of the reform of the functioning of secondary/supplementary public pension funds and ensures the unification into ETEA of all existing funds, which are considered to be in the domain of public sector according to ESA95 national account definition. (Q4-2012)

2. Ensures that the new single fund ETEA sets up, in a cost effective way, a computerised system of individual pension accounts; starting in Q1 2013 and to be finalised by Q42013.

3. Identifies the schemes for which lump sums paid on retirement are out of line with contributions and adjusts the payments. A new, actuarially neutral, formula to calculate lump sum, including a sustainability factor to avoid any future imbalances, is designed in consultation with the European Commission, ECB and IMF staff. (Q4-2012)

4. Will produce a regular quarterly report of the activities of the Health Committee, aimed at monitoring and revising the disability status and ensure that disability pensions correspond to not more than 10 percent of the overall number of pensions. (next report, Q1-2013)

\subsection{Modernising the health care system}

The Government continues to implement the comprehensive health sector reform with the objective of stabilising public health expenditure at, or below 6, percent of GDP, while maintaining universal access and improving the quality of care delivery. Policy measures include reducing the fragmented governance structure, reinforcing and integrating the primary healthcare network, streamlining the hospital network, strengthening central procurement and developing a strong monitoring and assessment capability and e-health capacity. 
The programme measures aim at achieving savings in the purchasing (accrual basis) of outpatient medicines of about EUR 1 billion in 2012 compared to 2011 and to reach spending of about EUR 2.440 billion in 2013 (accrual basis). The goal is to bring public spending on outpatient pharmaceuticals to about 1 percent of GDP i.e. around EUR 2 billion euro (in line with the EU average) in 2014. Total (outpatient plus inpatient) public expenditure on pharmaceuticals should be no more than 1.5 per cent in 2013 and 1.3 per cent in 2014.

\subsubsection{Governance}

a. Prior to the disbursement, to strengthen health system governance, improve health policy coherence, reduce fragmentation in the purchasing of health services and reduce administrative costs, the Government finalises the concentration of all healthrelated decision making procedures and responsibilities (including payroll expenditures) under the Ministry of Health by merging all health insurance funds, without exception, into EOPYY.

1. From January 2013, hospital services will be purchased directly by EOPYY through prospective budgets based on KEN-DRGs costing procedure (and payroll costs, should be at least reported).

2. EOPYY ensures that the number of doctors is reduced in headcount compared to June 2012 by at least 10\% by December 2012 and by a further 10\% in 2013.

\subsubsection{Controlling pharmaceutical spending}

In order to achieve EUR 1 billion of reduction in outpatient pharmaceutical spending in 2012 and reach the 1 percent of GDP target in 2014, the Government steps up its efforts, and further develops the set of incentives and obligations for all participants along the medicines supply chain (including producers, wholesalers, pharmacies, doctors and patients) to promote the use of generic medicines.

\subsubsection{Contingency measures to deliver the overall targets}

Prior to the disbursement, the Government:

a. adopts legislation which activates contingency measures (including e.g. a cross-theboard cut in prices or entry fee for the positive list), if, for any reason, the claw-back is not able to achieve the target. Such measures produce equivalent amount of savings.

b. sets, through Ministerial decree, the new claw back threshold for 2013, based on the above mentioned targets (Euro 2.44 billion for outpatients).

1. The Government revises the co-payment structure for medicines to exempt from copayment only a restricted number of medicines related to specific therapeutic treatments. (Q4-2012) 


\subsubsection{Pricing of medicines}

a. Prior to the disbursement, the Government repeals the current provision of the law which hampers the collection of the rebate from pharmacies in case of delays in payments on the part of EOPYY.

In addition, the Government:

1. Revises downward the price of medicines, based on the three EU countries with the lowest prices. In addition, the government re-prices medicines now cheaper than 10 EUR, including implementing a $10 \%$ price reduction in the prices of these medicines (quarterly update of price list in line with the provisions of Council Directive 89/105/EEC - next published by December 2012)

2. Applies an automatic claw-back mechanism (every six months) to pharmaceutical producers which guarantees that the outpatient pharmaceutical expenditure (EOPYY budget) does not exceed the above targets (Continuous).

3. Produces an implementation report on the impact of the new profit margins of pharmacies by Q1-2013 and shares it with the European Commission, ECB and IMF staff. If it is shown that this new model to calculate profit margins does not achieve the expected result of a reduction of profit margins down to $15 \%$, the regressive margin will be further revised.

4. Ensures that EOPYY negotiates a 5\% discount through price-volume agreements on medicines (200 medicines) (Continuous for 2013 and 2014)

5. Extends the application of the $5 \%$ rebate on pharmaceutical companies (which exists for hospital-priced medicines) to all products sold in EOPYY pharmacies (legislation adopted by Q4-2012).

\subsubsection{Prescribing and monitoring}

a. Prior to the disbursement, the Government updates the price list and the positive list of reimbursed medicines notably by reimbursing only the cost-effective packages for chronic diseases, by moving medicines from the positive to the negative and OTC lists and introducing the reference price system developed by EOF. These lists must be updated at least twice a year.

The Government will,

1. Extend the current e-prescribing to all doctors, health centres and hospitals. Eprescribing is made compulsory and must include at least 90 percent of all medical acts covered by public funds (medicines, referrals, diagnostics, surgery) in outpatient facilities and providers contracted by EOPYY and the other social security funds. (Q42012); the extension to NHS facilities will be finalised by Q2-2013. 
2. Implement the system (API) whereby pharmacies electronically register any residual manual prescriptions from doctors into the e-prescription application established by IDIKA. (Q4-2012);

3. Continue publishing prescription guidelines/protocols for physicians, with priority for the most expensive and/or mostly used medicines, and makes them compulsory (Continuous);

4. Enforce the application of prescription guidelines through the e-prescription system. (Q2-2013);

5. Further develop monitoring and control of e-prescription by introducing ICD-10 and SPC filters in the e-prescription system (Q2-2013);

6. Produce detailed monthly auditing reports on the use of e-prescription in NHS facilities and by providers contracted by EOPYY. These reports are shared with the European Commission, ECB and IMF staff teams. (Continuous);

7. Continue to provide a regular assessment of the information obtained through the eprescribing system. (Continuous);

8. Produce detailed quarterly reports on pharmaceutical prescription and expenditure which include information on the volume and value of medicines, on the use of generics and the use of off-patent medicines, and on the rebate received from pharmacies and from pharmaceutical companies. These reports are shared with the European Commission, ECB and IMF staff teams. (Quarterly updates);

9. Provide detailed reporting on individual prescription behaviour to each physician relative to the average of comparable (specialty, patient workload) physicians (both in NHS facilities and contracted by EOPYY and other social security funds until they merge) and signals when they breach prescription guidelines. This feedback is provided at least every month and a yearly report is published covering: 1) the volume and value of the doctor's prescription in comparison to their peers and in comparison to prescription guidelines; 2 ) the doctor's prescription of generic medicines vis-à-vis branded and patent medicines and 3 ) the prescription of antibiotics. (Continuous);

10. Enforce sanctions and penalties as a follow-up to the assessment and reporting of misconduct and conflict of interest in prescription behaviour and non-compliance with the EOF prescription guidelines (Continuous);

11. Select a number of the most expensive medicines currently sold in pharmacies, to be sold in hospitals or EOPYY pharmacies. (Q4-2012).

12. Implement a mechanism to reduce off-label prescription (Q4-2012)

\subsubsection{Increasing use of generic medicines}

Prior to the disbursement, the Government: 
a. Makes it compulsory for physicians to prescribe by international non- proprietary name for an active substance, with no reference to any brand name on the prescription form. This constitutes a major health reform. To avoid any potential risk to the health of the patient, brand name prescription will, however, be allowed in limited and duly motivated cases. The share of branded name prescriptions can be no more than $15 \%$ of the overall prescriptions of each doctor and the doctor needs to provide the relevant justification in each case. A strict control of the prescription of each doctor is implemented through the e-prescription monitoring system, using warning mechanisms to each doctor for when the prescription level by branded name is getting closer to the target. Prior to disbursement, a ministerial decree will explicitly define the exceptions to INN prescription, which must cover a very limited group of products (e.g. with narrow therapeutic index) and known sensitivities of the patient, according to international standards and best practices.

b. Mandates the substitution of prescribed medicines by the lowest-priced product of the same active substance in the reference category by pharmacies (compulsory "generic substitution").

The Government also:

1. Increases the share of the generic medicines to reach 35 percent of the overall volume of medicines sold by pharmacies by end-2012 and 60 percent by end-2013. This will be achieved by:

i. setting the maximum price of the generic to 40 percent of the price of the originator patented medicine with same active substance at the time its patent (exclusivity period) expired. After this first reduction, when exclusivity period expiry, further reductions are achieved through external reference pricing based on the three EU countries with the lowest prices. This will be done also by linking off-patent (when exclusivity period expires) products to the average of the three lowest prices in the $\mathrm{EU}$ and the price of the generic to $80 \%$ of the downward revised price of the off-patient. Further reductions are achieved through external reference pricing based on the three EU countries with the lowest prices. Producers are allowed to offer lower prices, thus allowing an increased competition in the market. (Continuous);

ii. automatically reducing the maximum price of originator medicines when their patent (exclusivity period) expires (off-patent branded medicines) to 50 percent of its price at the time of the patent expiry. Further reduction will be achieved by linking off-patent products to the average of the three lowest prices in the EU, to be revised periodically with price list. Producers can offer lower prices, thus allowing an increased competition in the market.

\section{(Continuous);}


iii. creating dynamic competition in the market for generic medicines through price reductions of at least 10 percent of the maximum price of each new generic producer entering the market. (Q4-2012);

iv. introducing (EOPYY) additional incentives and mechanisms, including a prescription quota system for physicians, to ensure generic substitution (Q42012);

v. deciding about the reimbursement of newly patented medicines (i.e. new molecules) on the basis of objective and strict medical and cost-effective criteria and, until internal capacity is in place, by relying on best practice health technology assessment of their cost-effectiveness carried out in other member states, while complying with Council Directive 89/105/EEC.

\section{(Continuous);}

vi. excluding from the list of reimbursed medicines those which are not effective or cost-effective on the basis of objective criteria. (Continuous);

2. Takes further measures to ensure that at least 50 percent of the volume of medicines used by public hospitals is made up of generics with a price below that of similar branded products and off-patent medicines. (Continuous)

3. Makes it compulsory for all public hospitals to procure at least $2 / 3$ of pharmaceutical products by active substance, using the centralised tenders procedures developed by EPY and by enforcing compliance with therapeutic protocols and prescription guidelines. (Q4-2012)

4. Adopts, with the pharmaceutical companies and physicians, a code of good conduct (ethical rules and standards) regarding the interactions between pharmaceutical industry, doctors, patients, pharmacies and other stakeholders. This code will impose guidelines and restrictions on promotional activities of pharmaceutical industry representatives and will forbid any direct (monetary and non-monetary) sponsorship of specific physicians (sponsorship should be attributed through a common and transparent allocation method), based on international best practice. (Q4-2012)

5. Speeds up administrative and legal procedures, in line with EU legal frameworks for the entry of cheaper generic medicines in the market. (Q4-2012)

\subsubsection{Reviewing the provision of medical services contracted by EOPYY}

a. Prior to the disbursement, to improve the current financial situation of EOPYY and ensure that the budgetary execution is closer to a balanced budget in 2012 and 2013, a set of measures will be implemented, including:

\footnotetext{
i. restricting the benefit package;

ii. increasing cost-sharing for private care;
} 
iii. negotiating price-volume agreements and revising case-mix agreements with private providers;

iv. revising the fees for and number of diagnostic and physiotherapy services contracted by EOPYY to private providers with the aim of reducing related costs by at least EUR 80 million in 2013.

v. introducing a reference price system for reimbursement of medical devices.

vi. progressively increasing the contributions paid by OGA members to the average of those paid by other members of EOPYY.

1. The government starts publishing a quarterly report on the prescription and expenditure of diagnostic tests. (quarterly updates - next report Q4-2012)

\subsubsection{National Health System (NHS) service provision}

\subsubsection{Reorganisation and management of the health care sector}

The Government:

1. Implements the plan for the reorganisation and restructuring, as set in Law 4052 / March 2012, with a view to reducing existing inefficiencies, utilising economies of scale and scope, and improving quality of care for patients, thus contributing to better aligning working organisation with Directive 2003/88/EC). This implies reducing hospital operating costs by 8 percent in 2012 and an additional 5\% in 2013 and reducing beds substantially, as legislated by MD OG1681/B (28-7-2011). This is to be achieved through:

i. increasing the mobility of healthcare staff (including doctors) within and across health facilities and health regions;

ii. adjusting public hospital provision within and between hospitals within the same district and health region;

iii. revising the activity of small hospitals towards specialisation in areas such as rehabilitation, cancer treatment or terminal care where relevant;

iv. revising emergency and on-call;

v. optimising and balancing the resource allocation of heavy medical equipment (e.g. scanners, radiotherapy facilities, etc.) on the basis of need.

vi. reducing administrative costs notably by removing deputy managers posts;

vii. reducing cost with outsourcing services such as IT services, laboratory services and hospital servicing costs (e.g. cleaning services). 
2. Produces an annual report comparing hospitals performance on the basis of the defined set of benchmarking indicators (Continuous)

3. Updates a report on human resources for the whole health care sector annually and uses it as a human resource planning instrument. (Continuous)

\subsubsection{Accounting, control, IT and monitoring systems}

The Government ensures that:

1. Internal controllers are assigned to all hospitals and all hospitals adopt commitment registers. (Q4-2012)

2. EOPYY publishes a monthly report with analysis and description of detailed data on healthcare expenditure with a lag of three weeks after the end of the respective month. This report will make possible the more detailed monitoring of budget execution, by including both expenditure commitments/purchases (accrual basis, by December 2012) and actual payments (cash basis). The report will also (1) describe performance on the execution of budget and accumulation of arrears, and (2) recommend remedial actions to be taken. (Continuous)

3. Further measures are taken to improve the accounting, book-keeping of medical supplies and billing systems, through:

i. the introduction of analytical cost accounting systems (Continuous);

ii. the regular annual publication of balance sheets in all hospitals. (Q2-

2013);

iii. the introduction of the uniform coding system for medical supplies developed by the Health Procurement Commission (EPY) and the National Centre for Medical Technology (EKEVYL) and the use of the observe.net system to monitor the procurement and use of tenders for medical supplies. (Continuous);

iv. the introduction of inbound hospital logistics and stock management (Q42013)

v. timely invoicing of full treatment costs (including staff payroll costs) - i.e. no later than 2 months to other EU countries and private health insurers for the treatment of non-nationals/non-residents. (Q4-2012);

vi. enforcing the collection of co-payments and implementing mechanisms that fight corruption and eliminate informal payments in hospitals.

(Continuous). 
4. ELSTAT starts providing expenditure data in line with Eurostat, OECD and WHO databases i.e. in line with the System of Health Accounts (joint questionnaire collection exercise). (Q4-2012)

5. The programme of hospital computerisation allows for a measurement of financial and activity data in hospital and health centres. Moreover, the Minister of Health defines a core set of non-expenditure data (e.g. activity indicators) in line with Eurostat, OECD and WHO health databases, which takes account of the future roll-out of DRG (diagnostic-related groups) schemes in hospitals. (Continuous)

6. The government starts to develop a system of patient electronic medical records. (Q12013)

7. In all NHS hospitals, the Government, with technical assistance from experts across EU, continue piloting a set of DRGs, with a view to developing a modern hospital costing system for contracting (on the basis of prospective block contracts between EOPYY and NHS). DRGs include a detailed item on costs of personnel. (Continuous)

8. An analysis will be made of how hospital accounting schemes integrate DRGs at hospital level in view of future activity-based cost reporting and prospective budgets payment for hospitals (Q4-2012)

\subsubsection{Centralised procurement}

1. The Government increases substantially the number of expenditure items and therefore the share of expenditure covered by centralised tender procedures through EPY. (Continuous)

2. EPY will undertake a major effort to utilise tender procedures for framework contracts for the most expensive medicines used in the outpatient context so as to substantially reduce the price paid by EOPYY. (Q4-2012)

3. In compliance with EU procurement rules, the Government conducts the necessary tendering procedures to implement a comprehensive and uniform health care information system (e-health system) including the full and integrated system of hospitals' IT systems. (Continuous)

\subsection{Upgrading the education system}

1. The Government implements the Action Plan for the improvement of the effectiveness and efficiency of the education system and regularly reports on the progress of its implementation including on the results of the external evaluation of high education institutions (December 2012).

2. On higher education: the provisions of the laws $4009 / 2011$ and $4076 / 2012$ are fully and promptly implemented including:

i. the activation of the Quality Assurance Authority (December 2012); 
ii. the constitution of the Council of Higher Education Institutions is completed (March 2013), with the respective organisation charters and internal regulations completed (September 2013);

iii. the election of the new Rectors (December 2012);

iv. the procedure on the consolidation/merging of departments of universities and technological institutes (ATHINA Project) starts to be implemented

(March 2013).

3. On primary and secondary education, progress on the implementation of the school and teacher evaluation policy including the schools' self-assessment project is reported on a quarterly basis (as of Q1 2013). In addition, more flexibility is introduced by end-December 2012 in the adjustment of tuition fees by private schools, as per Opinion 20/VI/2012 of the Hellenic Competition Commission.

\section{Stabilising the financial system}

\subsection{Recapitalisation of the banking sector}

The Bank of Greece (BoG) completed a strategic assessment of the banking sector in March 2012, assisted by an international consultancy firm. The study assessed the viability of the banks based on quantitative and qualitative criteria. This study identified four core banks accounting for approximately 75 percent of banking sector assets.

The authorities have reviewed their estimate of resources needed to fully recapitalize the Greek banking system. Based on the strategy laid out below, they estimate that the funds required will still amount to $€ 50$ billion, which is fully accounted for in updated estimates of program financing.

\subsubsection{Identification of capital needs}

a. Prior to the disbursement, the BoG has informed all banks, of their individual capital needs and has requested that they finalize the capital raising process by end-April 2013. The capital needs account for the impact of the valuation losses on new Greek government bonds, and results of a stress test exercise with a 3-year horizon (which took into account BlackRock credit loss projections and banks' future preprovisioning results).

1. By Q4-2012, the Government and the Bank of Greece will align capital metrics to the minimum core tier I capital ratio of 9 percent of risk-weighted assets set out in the European Banking Authority (EBA) recommendation on capital buffers. Banks will also have to meet the requirements set by the BoG under Pillar II (to maintain a 7 percent core tier 1 capital ratio under a 3 year adverse stress scenario).

2. The BoG will publish a detailed report on the individual banks' capital needs, recapitalization process and the methodology followed by Q4-2012. 


\subsubsection{Recapitalization process}

Current or new shareholders will have control of the core banks, provided they are deemed fit and proper as already envisaged in the regulatory framework, and have subscribed no less than 10 percent of the capital to be raised by way of common shares. While existing shareholders will be diluted during the recapitalization process, they or new investors will be allowed to participate in the rights issuance and should the above 10 percent threshold of private sector participation be reached, will receive warrants to acquire the remaining shares from the HFSF within five years.

Subsidiaries have been recapitalized by their parent banks. Agreements have been reached on the acquisition of Emporiki and Geniki by Alpha Bank and Piraeus Bank, respectively, with a view to achieve further consolidation of the banking system while protecting the public sector from potential losses. It is expected that the authorities will approve these acquisitions subsequent to the completion of the due diligence process. These acquisitions will not require injection of additional public funds.

a. Prior to disbursement, the legal framework for recapitalisation is put into place.

1. The recapitalization process of core banks will involve three broad steps:

i. First, the Hellenic Financial Stability Fund (HFSF) will provide sufficient funds in the form of bridge capital to bring the core banks up to the minimum level of 9 percent CT1 under Pillar 1 by end-December 2012. The HFSF will also issue a commitment letter to subscribe to 100 percent of the remaining capital needs.

ii. In the second step, by end-January 2013, the HFSF will subscribe to 100 percent of any convertible instruments that the banks will decide to issue.

iii. In the third stage, by end-April 2013, the core banks will complete the rights issue and any shares not subscribed by the private sector will be acquired by the HFSF subscription to the common equity.

2. The recapitalization strategy for remaining undercapitalized non-core banks has also been finalized. The strategic assessment of the authorities shows that these institutions are less suitable candidates for public money. These banks must be fully capitalized by end-April 2013. They may also merge with other banks if they can demonstrate a credible business plan, meet recapitalization needs by end-April 2013 and address all viability challenges.

3. The authorities are developing a strategy to address the on-going challenges for the cooperative banks. By end-February 2013 the BoG will complete its assessment of this sector and issue a final report. Based on this report, by end-May 2013, the authorities will set out a comprehensive strategy to implement its recommendations. 


\subsection{Framework for restructuring and strengthening of the banking system}

The government of Greece has established a framework to ensure continued restructuring and strengthening of the banking system after the recapitalization process is complete. Banks that receive state-aid will need to provide clear and realistic business plans for their restructuring:

1. Operational restructuring. Following the recapitalization of the core and non-core banks, all institutions should update their restructuring plans and submit them for validation by the EC. These should be finalized by Q2-2013. However, banks that acquire other institutions through P\&A transactions sponsored by the HFSF will have to submit such revised plans by end-July 2013. The restructuring plans should take into consideration the updated macroeconomic framework set out in the most recent program review. The HFSF will continuously monitor banks' adherence to their restructuring plans and report to the EC/ECB on progress on a semi-annual basis.

2. NPL resolution. The HFSF will request that banks assess whether their established frameworks and policies to deal with troubled assets are effective by June 2013. International work-out specialists should be invited to assist in the process.

3. Funding. As part of the new restructuring plans, banks will set out their intentions to broaden their funding base and reduce over time their reliance on emergency liquidity provided by the central bank. The BoG, following the procedures and rules of the Eurosystem, will stand ready to continue disbursing adequate and appropriate emergency liquidity support in a timely manner if needed. (Continuous)

4. The authorities will take no fiscal policy actions to increase the burden for the programme, and in particular will adjust the structure of outstanding governmentowned bank capital instruments (preferred shares) to ensure that they can continue to be counted as bank capital. A one-time $€ 550$ million fee, to be received from banks in 2012 in return for the provision of bridge capital, will be earmarked to the HFSF and placed in the HFSF intermediate account.(Continuous)

\subsection{Resolution of undercapitalized banks}

1. The authorities will complete the resolution of undercapitalized banks by mid-June 2013 and establish a framework to manage the assets of banks under liquidation:

i. $\quad$ State-owned banks. ATE bank was resolved in July through a promptly conducted Purchase and Assumption (P\&A) transaction with Piraeus Bank. The final resolution cost will be defined by an external audit of the transferred assets and liabilities. Additionally, Piraeus Bank has presented to the EC a restructuring plan to demonstrate that the new integrated firm will enhance its viability and its costefficiency. Regarding Hellenic Postal Bank, the authorities have initiated its orderly resolution with the aim to do so via a P\&A transaction to be completed no later than end-January 2013. Finally, the authorities will complete Nea Proton's restructuring by May 2013 under the sponsorship of the HFSF. 
ii. Other undercapitalized non-core banks. If the shareholders or new investors are unable to support these institutions as required above by end-April 2013 the authorities plan to complete the resolution of these institutions by end-June via P\&A transactions with well capitalized banks, or, as a second best, the establishment of a bridge bank. To prevent market distortions and unsound banking activities, the BoG has placed all undercapitalized non-core banks under enhanced supervision.

4. Greek authorities will ensure that the assets of banks under liquidation are managed on the basis of best international practices. This includes the bad assets of banks that are resolved as part of the restructuring process. To this end, by end-February 2013 the BoG will publish an assessment report prepared by an international expert regarding policies and procedures required to ensure effective bank asset management and recovery. The report will identify the areas that could require further strengthening to maximize loan collection and help reduce bank resolution costs.

\subsection{Safeguards to ensure stability and viability of the financial system}

The authorities are committed to ensure that the financial system operates with maximum safeguards to ensure stability and continued viability.

\section{Prior to disbursement:}

a. The HFSF will complete the due diligence of core banks and any findings of interest to the supervisor will be communicated to the BoG. The due diligence will, inter alia, focus on a review of governance including loans to related parties, asset quality, and risk concentration. The BoG will address these findings promptly, including suspension of private shareholders (which would prevent them from participating in bank recapitalization framework) and/or removal of board members and managers.

b. The authorities agree with the EC/ECB/IMF the terms of reference for the monitoring trustee and have communicated them to the banks with instructions for the trustees to begin work no later than mid-January 2013.

c. The authorities will amend the HFSF by-laws to clearly stipulate that the HFSF Board, including the EC and ECB observers at the HFSF, must be informed of all decisions of the core banks having an impact on the HFSF's rights as a shareholder/investor. This information, as soon as received by and through the senior executive of the HFSF, is to be provided within one day of receipt.

1. By Q1-2013 banks will submit to the BoG plans to address identified operational governance weaknesses with clear timetables for full implementation by Q4-2013.

2. Monitoring trustees will be appointed in all banks under restructuring to submit quarterly reports on governance and operations, as well as ad-hoc reports as needed. The monitoring trustees: 
i. Will work mainly under the direction of the $E C$, within the terms of reference agreed with the EC/ECB/IMF and will liaise closely with the EC/ECB observers at the HFSF and share their report with the HFSF. In line with the EU state aid rules the trustees will be responsible for overseeing the implementation of restructuring plans, including verifying proper governance and the use of commercial basis criteria in key policy decisions.

ii. Will follow closely the banks' operations and shall have permanent access to Board meetings' minutes, and be observers at the executive committees, and other critical committees including risk management and internal audit functions.

iii. Shall be a respected international auditing or consulting firm (that will include the participation of overseas based partners and managers) which needs to be endorsed by the EC on the basis of its competence, its independence from the banks and the absence of any potential conflict of interest.

3. The authorities will ensure adequate reporting of HFSF operations, and enhance the role of EC and ECB observers' access to information at the HFSF. Starting by endJanuary 2013, the HFSF will initiate semi-annual public reporting on its main activities.

4. The authorities will ensure arms-length governance of core banks' business activities. By Q1-2013, the HFSF will publish relationship frameworks with each bank on the basis of best international practices, with a view to define the responsibilities of bank managers and board members and the role of HFSF as a shareholder, to ensure the core banks are run on a commercial basis. A draft for discussion based on international best practices will be developed with the EC, ECB, and IMF staff by endJanuary 2013.

\subsection{Adaptation of banking supervision}

The BoG and the Government have maintained the stability and adequate supervision of the financial sector. With a view of adapting the supervision of the Greek banking sector to the changed banking environment the BoG are taking further important steps.

1. Updating the supervisory model. The BoG will complete a review of its supervisory approach in light of the new challenges ahead, by Q2-2013, with technical support provided by a banking supervision expert. Key enhancements will include: (i) the refocusing of off-site analytical capacity to assess the business models of the core banks and be able to monitor and critically analyse the implementation of their business plans and the direction that banks may be taking; (ii) updating onsite supervisory procedures and prudential regulation in light of the findings of the ongoing reviews; and iii) an action plan to monitor credit risk concentration, in line with best practices, such as enhancing the monitoring of large business groups (including those related to bank owners) by end-July 2013. 
2. Standardizing asset quality disclosure. To enhance information available on banks' asset quality, provisioning and income recognition, the BoG and the Hellenic Capital Markets Commission (HCMC) will issue guidelines in accordance with their respective competences, in order to align banks' disclosure practices to international best practices. This is particularly relevant regarding the accounting for provisioning and the reporting of restructured loans. By end-August 2013 with the assistance of a leading consulting firm, such practices will be benchmarked against those followed by top European institutions and will be reflected in the banks' end-2013 financial statements.

3. Undertaking a Basel Core Principles (BCP) assessment. The authorities will request the IMF to undertake a stand-alone assessment by Q4-2014. The BoG will prepare in advance by end-June 2014 a self-assessment of compliance with the BCP with the support of independent experts by Q2-2014.

4. Clarifying competences and responsibilities. The authorities will develop and publish a memorandum of understanding governing the relationship of the HFSF as a shareholder and the BoG's role regarding the oversight of banks that have received state aid.

\subsection{Review of insolvency frameworks}

The authorities will strengthen the insolvency framework.

1. In consultation with the EC/ECB/IMF staff, the authorities will, by Q4-2012, on the basis of best international practices, review the insolvency framework of households and SMEs as well as the framework for out of court negotiations between banks and troubled borrowers, and prepare an assessment identifying areas for improvement. The aim will be to achieve predictable, equitable and transparent allocation of risks among all interested parties and maximizing value for the economy in general.

2. By end-February 2013, the authorities will revise, with technical support of international experts, the existing framework to facilitate workouts with overindebted household borrowers that preserves bank solvency and credit discipline, avoids the use of fiscal resources to protect private borrowers and minimizes moral hazard by targeting borrowers that are in real need. The authorities will refrain from supporting initiatives that may undermine the payment culture in Greece.

\subsection{Follow up stress testing}

1. To ensure that the system remains sufficiently well-capitalized, the BoG will conduct a new stress test exercise, based on end-June 2013 data, using a methodology determined in consultation with the EC/ECB/IMF, by Q4-2013. 


\section{Strengthening labour market institutions and promoting employment}

The Government will build upon the recent labour market reforms, by promoting an efficient wage-setting system, reducing non-wage labour costs and creating more options for the adaptability of working hours. These reforms should support the on-going adjustment of the labour market, with the aim of reducing nominal unit labour costs in the economy by 15 per cent over the period 2012-14, and thus help restore cost-competitiveness and boost employment in the medium to long term.

The Government will fight undeclared work and informality, also by streamlining administrative burdens and increasing the transparency and enforceability of the labour law, and enhance policies in order to help the unemployed returning to paid jobs, while developing adequate and affordable social safety nets. In addition, within the context of the product and service market reforms in order to help the latter to succeed, labour legislation treating preferentially some sectors or professions will be brought in line with general standards applicable to the rest of the economy as presented in Section 6.

Reforms in labour legislation will be implemented in consultation of social partners as a rule, and in respect of EU Directives and Core Labour Standards.

\subsection{Reforms in the wage-setting system}

The wage setting framework at national level will be reformed in consultation with social partners to help ensure that wage dynamics support employment while setting a floor for labour income.

The reform shall aim at establishing a statutory minimum wage system as the nation-wide legally binding minimum floor for wage setting. Negotiations for labour agreements and contracts of any type and level shall take into consideration the binding floor of the statutory minimum wage rate. As of the enactment of this reform, the National General Collective Labour Agreement binds only the signatory parties, regarding wages, allowances and any other direct remuneration clauses. The minimum wage rate shall be legislated by the Government after consultation with social partners, other stakeholders and independent experts, taking into account the economic and labour market situation and prospects. The reform will also define how the economic and labour market situation and prospects will be factored into that process in order to ensure that the objectives of supporting employment and safeguarding labour income are achieved.

\section{Prior to the disbursement,}

a. The Government adopts the framework provisions for the reform of the minimum wage framework as described above, with the view of having the necessary changes finalised by Q1-2013 at the latest.

1. With this reform, the base wage and the maturity allowances currently linked to the National General Collective Labour Agreement will start by being set in a statutory way without exceeding their current level over the Programme period, with no other statutory minimum allowances. The statutory minimum wage system is expected to come by Q1-2013. 
2. By Q1-2014, the Government will review the minimum wage system, with a view to possibly improve its simplicity and effectiveness to promote employment and fight unemployment and help the competitiveness of the economy.

\subsection{Adaptability of working hours arrangements}

Work schedules shall be made more flexible in order to allow working hours to better adjust to demand and production patterns that may vary over time as well as over sectors and firms, and thereby help employment and competitiveness.

\section{Prior to the disbursement,}

a. The Government makes it possible - and in full respect of the current limits on the duration of the working week (including the 40-hours working week as a reference) and of minimum rests due to health reasons for specific categories of workers - to: (i) on a contractual basis, apply the general rules on the number of maximum workdays to sectors not now covered by the general rules; (ii) set the minimum daily rest at 11 hours; (iii) allow in seasonal sectors the consecutive minimum two week leave requirement to be taken anytime during the year.

\subsection{Reducing non-wage labour costs}

\section{Prior to disbursement,}

a. the Government reduces the maximum dismissal notification period to 4 months and caps statutory severance pay at 12 months (while preserving the existing link between tenure and severance for tenures with severance below the cap). If the cap has already been surpassed on the date of the reform, the amount accrued will be grandfathered in case of future dismissal any time thereafter, subject to a cap of EUR 2000 per month for the number of months exceeding 12. Occupations for which statutory severance costs are in excess of the rule just described, the compensation for severance will be aligned with the latter.

With a view to foster employment creation, the Government:

1. adopts legislation by November $\mathbf{2 0 1 3}$ reforming the system of social contributions, by broadening the base for contribution; simplifying the schedule across the various funds; shifting funds away from nuisance taxes and onto contributions; and reducing average contributions rates by 3.9 percentage points, which will be phased in over 2014, 2015 and 2016. The reform will be revenue neutral and preserve the actuarial balance of the various funds.

2. As intermediate steps, actuarial studies of possible changes in the system of social contributions will be carried out and action plans proposed by September 2013.

3. Carries out actuarial studies of first-pillar pension schemes in companies where the contributions for such schemes exceed social contribution rates for private sector 
employees in comparable firms/industries covered in IKA and presents options for the reduction of social contribution rates by Q2-2013.

4. Based on these studies of first-pillar pension schemes, and together with the reform of the system of social contributions, reduces social contribution for these companies and adjusts benefits in a fiscally-neutral manner by Q4-2013.

\subsection{Lowering compliance costs, fighting undeclared work and informality}

The Government streamlines reporting requirements on firms internal work arrangements in order to reduce the administrative burden - thereby lowering the costs of compliance and helping formality in labour arrangements, and reforms the Labour Inspectorate.

Prior to disbursement, the Government:

a. eliminates the obligations: i) to ex-ante submit work schedules to the Labour Inspectorate; ii) to require pre-approval by the Labour Inspectorate of: overtime work, itinerary books of trucks and buses, the work book of daily employment of construction workers, and split of annual leave. These changes shall not apply in the cases of underage employees and workers. Employers will be obliged to record this information and make it available to the Labour Inspectorate for checks whenever requested.

b. undertakes an independent external assessment of the Labour Inspectorate, to be completed by Q4-2012, on: (a) the mandate, activities and structure of the Labour Inspectorate with a view to increase its effectiveness and efficiency in fighting undeclared work while keeping administrative burdens for firms contained; and (b) the enforcement and penalty structure for infringements of labour arrangements (including undeclared work).

To implement the reform of the Labour Inspectorate:

1. The Government, based also on the external assessment of the Labour Inspectorate, shall present and start implementing a detailed action plan by February 2013, aimed at strengthening the fight against undeclared work and raising the effectiveness of the Labour Inspectorate. The plan should focus on: strengthening the mandate and effectiveness and efficiency of the Labour Inspectorate; amending monetary and legal penalties for infringement of law and regulations; streamlining the reporting by employers and employees; and reinforcing anti-fraud and anti-corruption mechanisms.

\subsection{More transparent and enforceable labour law}

1. The Government adopts by Q4-2013 a single Labour Code compiling all existing legislation relevant for labour and industrial relations. This should ease interpretation, reduce compliance costs and increase enforceability of labour law. By Q1-2013 the Government shall prepare a report on the structure of the Labour Code. 


\subsection{Support to the unemployed}

1. The Government has already taken measures and will further seek to prevent unemployment becoming permanent and to mitigate the hardship of unemployment, by focusing on: facilitating the transition of workers across occupations and sectors; improving the quality of training policies; promoting the employability of the disadvantaged groups; targeting segments of the population with the strongest need of income support. To this end, and in order provide continuing support to the Active Labour Market Policies, an Action Plan should be adopted by Q1-2013 focusing on:

i. Supporting job matching and activation of the unemployed by reforming and broadening the role of the Public Employment Service and by introducing shortterm public work programmes where feasible and appropriate;

ii. Enhancing the effectiveness and adequacy of measures for re-skilling the unemployed, including by promoting the training of the unemployed by firms;

iii. Facilitating the combination of reduced working time schedules with training in case of temporary reductions in activity;

iv. Enhancing unemployment benefits to help mitigate the short-term impact of unemployment and supporting the long-term unemployed and specific categories of workers without entitlement to unemployment insurance.

The action plan should provide a description of the programmes currently in force and plans for their rationalisation as well as an indication on sources of financing.

\section{Creating favourable conditions for economic activity}

The program places strong emphasis on implementing structural reforms that aim at improving the business and overall economic environment and contribute in enhancing competition and competitiveness. These include horizontal measures to reduce time and costs to i) create a company, ii) to get establishment and operating licenses for manufacturing activities, iii) to get permits for environmental projects and activities, iv) to export and import, combined with measures to improve the functioning of the judicial system.

\subsection{Promoting an efficient and competitive business environment}

\subsubsection{Rationalising / eliminating quasi-fiscal charges}

1. The Government further refines by November-2012 the list of non-reciprocating charges in favour of third parties presented to the Commission services in November 2011 by i) identifying beneficiaries; ii) specifying the legal base of each contribution and iii) quantifying contributions paid by consumers in favour of those beneficiaries.

2. Within the preparation of the $\mathbf{2 0 1 3}$ budget, the Government ceases to earmark and directs: 
i. the 0.5 percent charge provided for in the Emergency Statute $788 / 48$ and in Law 3883/1958 on the value of all imported merchandise in favour of the National Technical University of Athens, the University of Thessaloniki, the Athens Academy and for the promotion of exports.

ii. the non-reciprocating charge calculated on the fuel price in favour of Mutual Distribution Fund of the Oil-Pump Operators of Liquid Fuel.

3. The Government eliminates the vast majority of the quasi fiscal charges in a budget neutral way in the 2014 budget. (October 2013)

\subsubsection{Reducing procedural and other administrative burden}

1. To further simplify time for and reduce the cost of company creation,:

Prior to the disbursement, the Government,

a. removes the mandatory presence of a lawyer to draft the articles of association of companies with capital in excess of $€ 100,000$ (cfr. Art. 42.1 of law 3026/1954).

The Government, among others:

i. Eliminates the prior check carried out by the One Stop Shop (OSS) on outstanding social security and health payments by founders, members, and directors of the new company, so that it does not cause a potential blockage to company formation. Instead, and after company formation, the OSS would notify IKA and / or OAEE of the company number and founders information, so that IKA and / or OAEE can take any further actions that they deem necessary. (December 2012)

ii. Eliminates the ex-ante requirement for company seal and perforation of documents (books) as per the Code of Books and Records. (November 2012)

iii. Enables the Ministry of Development to issue a Ministerial Decision providing for draft model company statues without the prior proposal of the chambers of notaries and lawyers (cfr., Art. 12.2 of Law 3853/2010)

(December 2012) and publishes the model company statutes in January 2013.

iv. Reduces the minimum capital requirements of new companies to levels comparable with "best practice" Member States and within the limits of the second Company Law Directive. (December 2012)

v. Pass legislation making voluntary the registration of companies with the chambers of commerce as of January 2015. (December 2012) 
vi. Takes additional measures by June $\mathbf{2 0 1 3}$ at the latest, to reduce by $\mathbf{5 0 \%}$ the cost of starting a business, as measured by the World Bank's Starting a Business sub-indicator. The results will be monitored in the World Bank's 2014 edition of Doing Business. (November 2013)

2. To implement law 3982/2011 on the fast track licensing procedure for technical professions, manufacturing activities and business parks and other provisions, the Government issues the Presidential Decrees provided for in:

i. Art. 4.4 of the same law, regarding preconditions for obtaining a licence for industry technicians, plumbers, liquid and gaseous fuel technicians, cooling technicians and machine operators in constructions. (December 2012)

ii. Art. 4.4, on preconditions for obtaining a licence for electricians, to reduce the number of specialisms, increase the mobility of electricians within the same level category and provide for the institution of private certification bodies. (February 2013)

iii. $\quad$ Art. 27.4, on Certified Inspectors. (February 2013)

iv. Art. 24.5, laying down the requirements for Citizens Service Centres' (KEP) for integrated electronic application among licensing bodies and for modalities of processing demands through it. (March 2013)

v. Art. 27.4, defining the fees paid for establishment and operating licenses. (December 2012)

vi. Art. 35.2, providing for the certification of the licensing service of the Chamber of Engineers. (December 2012)

3. To implement Law 4014/2011 on environmental licensing of projects and activities, the Government issues the Ministerial Decisions provided for in:

i. Art. 2.13, to further specify the procedure and specific criteria for environmental licencing. (December 2012)

ii. Art. 8.3, on environmental licensing of projects and activities (other than industrial activities), laying down the standard environmental commitments of projects and activities in category B. (January 2013)

4. To simplify export and import procedures, the Government:

a. Prior to disbursement, amends the regulatory framework of custom brokers to, among others i) eliminate the limitation of customs brokers' services to natural persons and to legal partnerships; ii) allow legal persons to represent others at customs, authorise companies (natural and legal persons) to complete customs formalities (for themselves or for other companies) without employing the services of a customs broker; iii) lift 
geographical restrictions, nationality requirements, the age limit to sit the examinations for customs brokers; iv) increase the frequency of examinations; v) review the system of annual renewal of licenses and to vi) repeal minimum fees.

In the short term, the Government:

i. Publishes a National Trade Facilitation Strategy (October 2012) with time bound quantitative performance indicators to streamline pre-customs and customs procedures according to best practices and EU regulations. The overall goal of the strategy is (i) to establish an electronic single window for exports providing for a single entry point for exporters for all products and destinations and (ii) to achieve $50 \%$ reduction in the time needed to export by Q4 2015.

ii. Launches in November $\mathbf{2 0 1 2}$ a review of the customs office as a pilot to optimise operations, reduce the number of physical and documentary controls, reassess the organization of labour, sequencing of procedures and work shifts and to enable 24/7 trade. The review is completed in March 2013 and is applied to Athens airport and Piraeus Port by (March 2013) and all other customs offices by December 2013.

iii. Reviews the risk assessment system for exports, with recommendations for improvement in order to align it with best practices in EU Member States. (December 2012) The level of controls converges to the average level of controls in the EU by September 2013.

iv. Reviews and streamlines pre-customs and customs procedures for selected pilot products (fresh vegetables fruits, white cheese) according to EU regulations and best practices and presents an approach for extending the simplification process to a wider set of frequently exported / imported products. (December 2012)

v. Ensures that the e-customs system supports the electronic submission of all import and export declarations, including the possibility of submitting documents and making payments electronically. (June 2013)

vi. Implements automatic clearance for low risk declarations, based on EU best practices. (December 2013)

vii. Ensures that the trade facilitation strategy is supervised and coordinated by an inter-ministerial steering committee, to be appointed by December 2012.

5. To identify and eliminate unnecessary reporting requirements for businesses: 
i. The Government, assisted by the OECD, starts applying the Standard Cost Model (SCM) to identify administrative burdens for businesses in 13 key sectors, with a view to reducing administrative burdens by at least 20 percent (compared with the baseline year 2008). (December 2012)

ii. The sectors are the following: Agriculture and agricultural subsidies, Annual accounts/company law, Energy, Environment, Fisheries, Food safety, Pharmaceutical legislation, Public procurement, Statistics, Tax law (VAT), Telecommunications, Tourism, Working environment/employment relations.

iii. Following the identification of administrative burdens (by June 2013), the Government completes the amendments to sector specific legislation by September 2013.

7. To facilitate spatial planning including through an effective land registry, the Government:

i. adopts legislation to (i) simplify and reduce the time needed for town planning processes (January 2013); (ii) update (March 2013) and codify legislation on forests, forest lands and parks (September 2014). It also licenses at least two disposal sites for hazardous waste (June 2013).

ii. completes the revision of the 12 regional spatial plans to make them compatible with the sectoral plans on industry, tourism, aquaculture and renewable energy. The first phase of this revision is completed (December 2012), followed by a second phase for modifications (May 2013), a third phase for the formulation of proposals (September 2013) and a fourth phase for the legislation of the final proposal (December 2013).

iii. accelerates the completion of the land registry, with a view to:

a) tendering out all remaining rights (ca. 15 million) and awarding cadastral projects for 7 million rights. (December 2012)

b) digitalising the operations of all mortgage and notaries' offices and conveying all newly registered deeds to the cadastre by 2015 .

c) exclusively-operating cadastral offices for large urban centres by $\mathbf{2 0 1 5}$.

d) establishing a complete cadastral register and exclusively operating cadastral offices nationwide by $\mathbf{2 0 2 0}$.

\subsubsection{Enhancing competition}

1. With the help of the Hellenic Competition Commission, the Government will screen the restrictions in the air transport services (including airport services) and maritime transport (including seaport services) and will prepare by end-January 2013 a set of measures to promote competition in those sectors and facilitate price flexibility. 
2. The Government, assisted by the OECD, starts applying the Competition Assessment Toolkit in sectors such as food processing, retail trade, building materials and tourism to identify unnecessary restraints on market activities and develop alternative, less restrictive measures that still achieve government policy objectives. (December 2012)

3. Immediately upon completion of the analysis (June 2013), the Government will prepare the legislative amendments to remove disproportionate regulatory restrictions identified by the Competition Assessment Toolkit, with a view to having them adopted by September 2013.

\subsection{Reforming the judicial system to support economic activity}

To improve the functioning of the judicial system, which is essential for the proper and fair functioning of the economy, and without prejudice to the constitutional principles and the independence of justice, the Government:

- ensures effective and timely enforcement of contracts, competition rules and judicial decisions;

- increases efficiency by adopting organisational changes to courts;

- speeds up the administration of justice by eliminating backlog of court cases and by facilitating out-of-court settlement mechanisms.

In designing and implementing the measures below, the Government consults the EC/IMF/ECB.

\subsubsection{Review of the code of civil procedure}

1. The Government commits to review the Code of Civil Procedure in accordance with the roadmap defined in section 9.3 of this Memorandum, which defines intermediate steps towards its completion by March 2014.

\subsubsection{Judicial statistics}

1. In order to facilitate the implementation of a performance and accountability framework for courts, the Government will compile and publish on its website the information indicated in Section 9.4 of this Memorandum. (Quarterly)

2. It also establishes an interservice group between the Ministry of Justice, Transparency and Human Rights, the Ministry of Finance and the Hellenic Statistical Authority to cooperate in the compilation and publication of the data in Section 9.4 of this Memorandum and the establishment and updating of a database with case data for each court. This cooperation takes into consideration current and future e-justice applications. (November 2012) 


\subsubsection{Tax case backlog reduction}

1. Following the submission of the work plan for the reduction of the backlog of tax cases in all administrative tribunals and administrative courts of appeal in January 2012 and its first update in July 2012, which provides for the intermediate target for reducing the backlog by at least 80 per cent by end-December 2012 and for the full clearance of the backlog by end-July 2013, the Government, by end-October 2012 and thereafter once a quarter:

i. presents updated and further refined work plans (ensuring that priority is placed on high value tax cases - i.e., exceeding $€ 1$ million);

ii. takes remedial action in case of anticipated or actual deviations, and

iii. publishes reports on progress in backlog reduction.

\subsubsection{Non-tax case backlog reduction}

1. The Government presents to the EC/IMF/ECB and publishes the study of the backlog of non-tax cases in courts conducted jointly with an external body of experts. (December 2012)

2. By end-January 2013, the Government presents to EC/IMF/ECB, based on the abovementioned study, an action plan with specific measures for a reduction of such backlog of at least 50 per cent by end-July 2013.

\subsubsection{Reorganization of the magistrates' court}

1. As publicly announced, the Government adopts a Presidential Decree providing for the rationalisation and reorganisation of the magistrates' courts and the allocation of appropriate human resources and infrastructure for the new structure of magistrates' courts resulting from this reform making the most effective use of the existing resources available within the system. (October 2012) The Presidential Decree on the reform of the magistrates' courts is implemented by March 2013.

\subsubsection{Development of e-justice applications in courts}

1. By end-December 2012, the Government updates, further refines and operationalises every quarter the e-justice work plan of December 2011 and its first update of July 2012 for the use of e-registration and e-tracking of the status of individual cases in all courts covered by the action plan and for e-filing.

2. The updates will contain an evaluation to be completed by end-March $\mathbf{2 0 1 3}$ of the efiling pilot project at the Athens Court of First Instance, an evaluation to be completed by end-March 2014 of the level of integration of IT systems achieved at that time in all courts of the country, and a timetable with proposed deadlines aiming at the extension of e-registration and e-tracking to all courts by end-2015. The Government ensures consistency of the e-Justice action plan with the e-government strategy. 


\subsubsection{Promotion of pre-trial conciliation and mediation}

1. By end-October 2012, the Government develops its strategy on the active promotion of pre-trial conciliation, mediation, and arbitration, with a view to ensuring that a significant amount of citizens and businesses make use of these modes of alternative dispute resolution. In order to implement this strategy, the Government, inter alia, applies to obtain funding under the Operational Programme 'Administrative Reform' by end-November 2012.

2. By end-September 2013, the Government conducts an assessment of whether the enactment of Law 3898/2010 on mediation in civil and commercial matters has delivered the results which the legislation intended to achieve, and presents data and analysis concerning costs, time and success rates associated with the enforcement of agreements arising from alternative dispute resolution as compared with the enforcement of judicial decisions.

3. By end-December 2013, the Government presents a legislative proposal for the effective opening of the mediator's profession to non-lawyers.

\subsubsection{Other measures on judicial reform}

1. Assessment of the Law on fair trial and conciliation (4055/2012): The Government conducts an assessment whether the enactment of Law 4055/12 has delivered the results which the legislation intended to achieve, in particular as regards civil courts, improved case processing in multi-member first instance courts, the speeding-up of the issue of provisional measures, the strengthening of the institution of 'voluntary jurisdiction' in certain matters at the level of the magistrates' courts and efficiency gains in enforcement proceedings, and as regards administrative courts, the strengthening and general application of pilot proceedings in the Council of State and the speeding-up of the issue of provisional measures. (December 2012)

2. Administrative review of cases: The Government prepares, in accordance with Law $4048 / 2012$ on better regulation, draft legislation providing for, where appropriate, a compulsory administrative review before an independent committee before a case may be brought before the administrative courts and submits it to the Greek Parliament. (June 2013)

4. Study on the costs of civil litigation: the Government completes a study on the costs of civil litigation, its recent increase and its effects on workload of civil courts, together with recommendations. (June 2013)

\section{Efficient Network Industries and Services}

\subsection{Energy policy}

Designing an appropriate energy policy, given the country's needs and potential, is fundamental for economic growth. For this reason the Government must follow a holistic approach which 
maximises the benefits for market participants, protects consumers and vulnerable parts of the society and the rights of tax payers. The reforms below aim at bolstering competitiveness and efficiency in the electricity and gas market, to avert a repetition of the 2012 liquidity crisis and to position the energy sector as a potential contributor for prosperity.

\subsubsection{Unbundling effectively network activities from supply activities}

1. Legislation is passed providing for the details of the ITO-option for the gas TSO. (endNovember 2012)

2. DESFA applies for certification to RAE. (December 2012)

3. The unbundled gas TSO is certified by the Greek energy regulator. (March 2013 or prior to the clearance of the privatisation of DEPA, whichever date comes earlier)

\subsubsection{Provisions regarding the privatisation of PPC and DESFA:}

1. The Government submits a plan for the restructuring of PPC with a view to preparing the company for privatisation and to allowing PPC to be competitive with other firms in a liberalised electricity market. The plan shall specify which parts of PPC should be privatised and within which timeframe. The plan shall also include provisions for the divestment of lignite-fired and hydro electricity generation capacity currently managed by PPC and evaluate the possibility of ownership unbundling of ADMIE. (November 2012)

2. The Government undertakes that whichever the outcome of the privatisation process the gas industry and electricity industry structure will be fully compliant with Directive 2009/73/EC and 2009/72/EC. (Continuous)

\subsubsection{Ensuring that electricity prices reflect costs}

1. The Government takes measures for the gradual phasing out of regulated electricity prices for all but vulnerable customers by June 2013. In this respect:

i. it issues a Ministerial Decision to adjust end-user prices for low voltage customers, effective as of January 2013. (December 2012)

ii. as necessary, it issues a Ministerial Decision by end-March 2013, effective as of May 2013, to further set low voltage end user prices at cost recovery.

iii. it removes regulated tariffs for all but vulnerable consumers. (June 2013)

2. In addition, the Government assesses best practices with a view to charging royalties for the use of hydro and lignite. (March 2013)

3. To evaluate the incumbent electricity company's operating costs the Government submits to the EC/IMF/ECB and publishes a study by a specialist of European standing to compare PPC worker's remuneration with best-practice European electricity 
companies, by comparable job assignments and productivity per employee.

Remuneration and promotion schemes also to be assessed against practices in private Greek companies. (December 2012)

\subsubsection{Implementing smart metering systems}

1. The Government issues a Ministerial Decision, based on the advice of RAE, on the large-scale replacement of existing systems with smart metering systems and explores assistance from EU structural funds and/or the EIB to conduct this strategic investment. (end-December 2012)

\subsubsection{Providing for a financially sustainable development of renewable energy sources}

Within a short term perspective, the Government:

1. Ensures that from January $\mathbf{2 0 1 3}$ onwards, LAGIE publishes monthly data on the evolution of the RES account with projections throughout 2013. (Monthly)

2. Takes measures to reduce the accumulation of licences issued for renewable energy installations, but not implemented. (December 2012)

3. Adjusts the RES levy every six months (January and July 2013) to eliminate the projected RES account debt by end-December 2013.

To achieve a long-lasting reform of renewable support schemes, the Government:

4. Submits to the Commission services a detailed plan for the reform of the renewable energy support schemes such that they are more compatible with market developments and are subject to budgetary discipline. (December 2012) The reform should look into:

i. options applicable to the support scheme, including market elements such as forward looking tariff digression and feed-in-premia, combined with a ceiling on the number of licenses, volume of capacity supported or overall subsidies to be provided per technology/tariff category.

ii. current and expected trends in costs for all relevant technologies.

iii. a sustainable and transparent framework for the regular adjustment of the RES levy.

5. Caps temporarily new connections of rooftop PV systems. (March 2013)

6. Adopts the reform of the renewable energy support schemes as specified in paragraph 4. (June 2013) 


\subsubsection{Planning the development of the electricity market in the medium to long term}

1. A detailed plan and roadmap to change the market model is presented to the Commission services, including measures to have effective competition in generation and supply, the development of a power exchange, introducing an intra-day market and implementing market coupling with neighbouring markets. (end-December 2012)

2. The study also presents a time bound roadmap and estimation of costs of connecting Crete to mainland Greece. (end-December 2012)

\subsubsection{Fuel distribution}

1. To facilitate the import and trade of oil and oil products, in the transposition of Directive 2009/119, the Government creates the framework for setting up of a Central Stockholding Entity (CSE), within the meaning of the Directive. In addition, the transposition law will grant economic operators, on which it imposes stockholding obligations, the right of delegation of such obligations with:

i. the CSE in Greece (should there be one) or to other economic operators within Greece which have surplus stocks or available stockholding capacity, without quantitative restrictions, and /or

ii. the CSEs of other EU Member States up to a percentage of the stockholdings obligations imposed on them, upon the entry into force of the transposition law, and /or

iii. economic operators abroad which have surplus stocks or available stockholding capacity up to a percentage of the stockholdings obligations imposed on them, upon the entry into force of the transposition law.

The percentage of cases (ii) and (iii) is at least 30\%. (December 2012)

2. To eliminate restrictions on public and private use trucks for fuel distribution:

Prior to the disbursement, the Government takes measures to:

a. allow independent gas stations to own or rent tanker trucks of any capacity provided that safety standards for the transportation of fuel are respected.

b. allow independent gas stations to hire public-used tanker trucks for fuel transportation without needing to qualify for their own private used tankers.

c. allow any tanker truck, regardless of its capacity, to enter the refineries and customs installations to transport fuel under their own brand 
name/trademark, provided that safety standards for the transportation of the fuel are respected.

3. To improve monitoring systems, prevent illegal trade and tax evasion in fuel distribution:

\section{Prior to the disbursement, the Government:}

a. issues technical specifications and next steps for the implementation of the input-output measurement system in all fuel stations;

b. issues a Ministerial Decision providing for the installation of GPS systems, as provided for in Art. 320 of law 4072/2012.

\subsection{Electronic communications}

The switchover from analogue to digital TV technology will release a significant amount of high quality radio spectrum which will be free for the deployment of new services and new technologies. This 'digital dividend' can boost both the broadcasting sector and the wireless communication industry, make a major impact on competitiveness and growth, and provide a wide range of social benefits. The items below provide a roadmap for the release of the digital dividend in Greece.

1. Regarding the release of Digital Dividend, the Government (and/or EETT) undertakes to:

i. amend the frequency and the broadcasting plans, taking into consideration the state of play of international coordination, allocating and authorising the use of the digital dividend to Electronic Communication Services.

(December 2012)

ii. adopt necessary secondary legislation for the assignment of licenses for broadcasting and for the establishment of licensing procedures. (March 2013)

iii. launch the public consultation on the tender procedure for the assignment of the digital dividend ( $800 \mathrm{MHz}$ band) allocating and authorising the use of the digital dividend to Electronic Communication Services, in line with EC Decision 2010/267/EU and in respect of the deadlines and procedures of the RSPP. (December 2012) (*)

iv. resolve cross-border coordination issues with neighbouring countries, if any. If difficulties on international coordination make this date unfeasible, the frequency and broadcasting plans might indicate alternative channels for re-location of broadcasters, while continuing negotiations with third 
countries in view of the final assignment of frequencies to broadcasters and mobile operators. (Continuous)

v. Adopt secondary legislation that defines a mandatory date for the switchoff of analogue broadcasting and a technologically neutral utilisation of the $800 \mathrm{MHz}$ band after the switch off, taking also into account the provisions of the draft Radio Spectrum Policy Programme (RSPP). (March 2013)

vi. launch the tender for the assignment of rights of use for broadcasting transmission. (March 2013)

vii. proceed to the tender procedure for the assignment of frequencies of the digital dividend, allocating and authorising the use of the digital dividend ( $800 \mathrm{MHz}$ band) to Electronic Communications Services in line with EC Decision 2010/267/EU and in respect of the deadlines and procedures of the RSPP. (June 2013) (*)

(*) The deadlines for steps (iii) and (vii) might be amended according to the Decision of the European Commission on the request for derogation from the deadline provided by Art. 6.4 of the Radio Spectrum Policy Programme submitted by the Greek Government on 15 May 2012.

\subsection{Transport}

The opening of the road haulage and occasional passenger transport is completed. Therefore, priorities now shift to measures that allow for higher levels of tourism and investment, particularly with respect to road (limousines and shuttle services), maritime and port activities (domestic ferry and port services) and aviation. Specific actions are expected to lead to the reduction of operating costs of service providers, while increasing consumers' choice. The gradual restructuring of railways should also lead to its effective privatisation.

\subsubsection{Road}

Prior to disbursement, the Government adopts all necessary measures to:

a. Remove restrictions on the rental of pickup trucks, vans and chauffeur services;

b. Allow shuttle services by hotels and tour agencies using small vehicles (less than 12 seats) and tour packages for small vans and off-terrain vehicles.

1. After having completed the report on the functioning of the regular passenger services, the Government:

i. Defines a follow up strategy for the effective opening of the sector in line with state aid rules and EU Legislation on awarding contracts of passenger transport services, while ensuring continuity of service provision and identifying concrete options for public transportation in remote areas (December 2012). 
ii. Approves the required law (and necessary acts) that ensures equal conditions and access equity to all road passenger service operators (March 2013).

\subsubsection{Maritime Activities and Ports}

1. The Government:

i. Launches (October 2012) a consultation procedure to review the legal framework of the domestic ferry industry, with a view to strengthening the growth potential of the tourist sector. Particular attention is given, inter alia, to increasing the flexibility of (i) manning requirements of vessels outside the minimum routing obligations and (ii) approving changes in the timetables and vessel size for ferry services.

ii. Make a concrete amendment of Law 2932/2011 building on this consultation (February 2013).

iii. Launches the social dialogue, following the Maritime Labour Convention (2006) principles, in order for labour arrangements to respond to the current economic context and future developments (October 2012).

2. Following the first submitted version of the port national strategy, the Government adopts a five-year port national strategy that ensures connectivity of ports with the overall transport network, specifying concrete projects, deadlines for implementation and a financial plan. The strategy will be coherent with TEN-T priorities and principles, such as the smooth operation of the internal market, the mobility of persons and goods and the economic, social and territorial cohesion of the European Union, the efficient use of the assigned Structural and Cohesion Funds and in line with State aid rules (December 2012).

3. In alignment with the new ports strategy and organisational model for ports, the Privatisation Fund (HRADF) defines a concrete privatisation strategy mainly through concessions with the objective of making the best use of the ports (February 2013) and launches the appropriate call(s) for tender (June 2013).

4. The Government revises the legal framework governing port labour relations and the administrative functioning of ports and sends a draft proposal to the Commission services (see Annex 9.5 for a non-exhaustive list of labour regulations for ports) (December 2012). This revision complies with the EU social acquis and provides, among others, for the training and certification of cargo-handling employees, and defines a competitive model for ports and terminal operators. The new legal framework is adopted (March 2013). 
5. The Government examines compatibility with Community legislation and in particular State aid rules and amends as necessary provisions concerning Piraeus Port (OLP) and Thessaloniki Port (OLTH), in particular (December 2012):

i. Law 2688/1999:

a. Chapter A, Article Second, paragraph 2;

b. Chapter A, Article Third, article 5, paragraph 5;

c. Chapter B, Article Seventh, paragraph 2;

d. Chapter B, Article Eighth article 5, paragraph 5.

ii. Law 2937/2001: Article 34.

\subsubsection{Aviation}

1. After having submitted the National Airport Policy, the Privatisation Fund (HRADF) launches the appropriate process leading to the privatisation of regional airports (March 2012).

2. The Government submits legislation for the restructuring of the Hellenic Civil Aviation Authority (HCAA), which strengthens its regulatory role and implements the full separation between Regulation and Management from Operation. The Government ensures improvements in the performance of air traffic management delivery through adherence to the relevant EU Regulation (EU 691/2010) (December 2012). Public airports that will not be privatised will be formed under a public body and their management and operation will be totally separated from the HCAA. New legislation is adopted (March 2013).

\subsubsection{Railways}

1. The Government spins-off ROSCO (Maintenance Unit), GAIOSE (Real Estate), transfers the leasing of the rolling stock activities from the OSE Group to the State, and provides an updated TRAINOSE Business Plan (November 2012).

2. The Government amends the law such that the fee of charges for obtaining a license or a safety certificate does not exceed the average European charge. All operators are awarded safety certificates on the basis of published, simple and transparent rules for the duration of five years (December 2012).

3. The rolling stock that is not used/needed by TRAINOSE is transferred to a body that leases it on market conditions, including winners of such tenders (December 2012).

4. The Government establishes independent award authorities for passenger services by rail that can organize competitive tenders (January 2013). Contracts concluded in 2014 or later will generally be awarded by means of competitive tender. The National 
Safety Authority establishes the examination of five examiners for train drivers according to the EU Regulation and publication of register of examiners on the web. The safety authority publishes the conditions and procedures to recognize drivers (March 2013). The Government amends the safety legislation to establish a right of any company on access to train driver facilities and examiners (April 2013).

5. Tariffs in OSE Group and TRAINOSE (June 2013) and OASA Group (October 2013) increase by at least 25 percent.

\subsection{The Retail Sector}

On retail, the selected measures aim at allowing a wider class of goods to be sold by more efficient retailers, and reduce their operating costs. Measures not only look into retail specific regulations (such as rules on pricing, sales and labelling) but also, into the rules on transport and healthcare provisions applicable to retail outlets. Combined, the measures should help contribute to lower prices and more choice for consumers.

\section{Prior to the next disbursement, the Government:}

a. eliminates the requirement of minimum space for the sale of food products.

b. allows mixed shops to sell goods other than food, subject to hygiene and food and safety standards.

c. allows supermarkets the sale of pre-packaged meat, cheese and fish products;

d. liberalizes sale in supermarkets of infant milk (i.e., 0-6 months)

e. allows a delink of the working hours of all employees in establishments (as defined in Law 1037/1971 and related implementing legislation) from opening hours of the establishment.

f. adjusts the law to clarify that shift breaks are allowed in all retail establishments (including those with continuous working schedule).

In addition, the Government:

1. applies the same standards for transporting perishable goods to private-use trucks as those applicable to public-use trucks. (December 2012)

2. carries out a proportionality analysis of the restrictions applied on outdoor / ambulant trade for social policy criteria. (December 2012)

3. completes the revision of Ministerial Decision A2-3391/2009 on market regulations and submits it to the Commission services, in accordance with the notification procedure provided for in Directive 98/34. (October 2012) The revised Ministerial 
Decision on market regulations is adopted one month after the reply from the Commission services, following the notification process.

4. reviews and amends the Market Policing Code (Law 136 /1946) providing for various forms of public sector intervention in the production, distribution and consumption of goods in line with the simplifying recommendations of the Hellenic Competition Commission's opinion no. 24/VII/2012. (January 2013)

\subsection{Regulated professions, professional qualifications and provision of services}

\subsubsection{Removing restrictions to the access to and exercise of regulated professions}

a. Prior to the disbursement, the Government amends specific legislation to lift the restrictions in the professions and economic activities listed in Section 9.2.1 of this Memorandum.

1. For professions and economic activities included in Section 9.2.2, the Government prepares draft provisions amending sector specific legislation as per the opinions of the Hellenic Competition Commission. The legislation is adopted by December 2012.

2. A report on the implementation of Law 3919/2011 is published on the Government's website (December 2012), including:

i. the list of all professions/economic activities falling under the scope of that law.

ii. the list of all remaining professions/economic activities that have not been treated beforehand. For these, the Government ensures that the regulatory framework is fully in line with chapter A of law 3919/2011 and the opinions (if applicable) of the Hellenic Competition Commission (HCC) by

(December 2012).

3. To reinforce transparency in the functioning of professional bodies: Legislation is adopted mandating the publication on the webpage of each professional association the following information (December 2012):

i. the annual accounts of the professional association.

ii. the remuneration of the members of the Governing Board broken down by function.

iii. the amounts of the applicable fees broken down by type and type of service provided by the professional association as well as the rules for their calculation and application.

iv. statistical and aggregate data relating to sanctions imposed, always in accordance with the legislation on personal data protection. 
v. statistical and aggregate data relating to claims or complaints submitted by consumers or organisations and the reasons for accepting or rejecting the claim or the complaint, always in accordance with the legislation on personal data protection.

vi. any change in the professional codes of conduct, if available.

vii. the rules regarding incompatibility and any situation characterised by a conflict of interests involving the members of the Governing Boards.

4. To de-link minimum fees for professional services from taxation and contributions to social security funds and professional associations:

i. The Government issues a Presidential Decree, which sets a system of prepaid fixed/contract sums for each procedural act or appearance by a lawyer, which is not linked to a specific 'reference amount'. (November 2012)

ii. Within the context of the tax reform, it also identifies ways of de-linking taxation from engineers legal fees and from lawyers legal fees / reference amounts. (December 2012) This reform becomes operational upon the entry into force of the tax reform in January 2013.

5. To assess the proportionality and justification of activities reserved to specific regulated professions:

i. The Government updates the study of the regulations of the professions submitted to the Commission services in July 2012, to assess the justification and the proportionality of the requirements reserving certain activities to providers with specific professional qualifications. (November -2012)

iii. Draft legislation is sent to Parliament amending unjustified or disproportionate requirements reserving certain activities to providers with specific professional qualifications, starting from the main regulated professions (i.e., lawyers, engineers, accountants, sworn-in valuers, energy / building inspectors, etc.). (March 2013)

\subsubsection{Additional measures}

Prior to disbursement, legislation is adopted to:

a. repeal the mandatory involvement of a lawyer for transactions on ships with immediate effect and for the drawing up of real estate transactions with an immediate effect for the seller and with an effect from 31-12-2013 for the buyer, with the relevant thresholds immediately raised to $€ 80,000$ for the entire country; 
b. repeal the presence of a lawyer (with immediate effect) for the contracts for the distribution or exchange of real estate property as well as for the gratuitous legal transactions, such as donations;

c. repeal the scale of minimum monthly amounts that are due to private sector lawyers remunerated for services rendered with a fixed periodic fee. This is without prejudice to having fee regulations for trainee lawyers and the minimum wage for private sector workers provided for in law 4046/2012.

Other actions

1. A draft code revising Legislative Decree 3026/1954 is submitted to the Commission services by end-November 2012 and adopted by December 2012. The new code should, among others, abolish total bans on commercial communications, repeal age limits to take the Bar examinations, ease the re-entry into the legal profession and clarify the nature of lawyers' fees provided for in current legislation.

2. The Government takes additional measures by June $\mathbf{2 0 1 3}$ at the latest, to reduce by $50 \%$ the cost of registering property, as measured by the World Bank's Registering Property sub-indicator. The results will be monitored in the World Bank's 2014 edition of Doing Business. (November 2013)

3. To confirm progress in the area of regulated professions, the Government completes a study of the 20 largest professions examining the degree to which they have been liberalized, including results with respect to new entrants and price changes. (July 2013)

\subsubsection{Easing the recognition of professional qualifications}

1. Measures are taken to ensure the effective implementation of EU rules on the recognition of professional qualifications; including compliance with $\mathrm{ECJ}$ rulings (inter alia, related to franchised diplomas). In particular, the Government:

2. continues to update the information on the number of pending applications for the recognition of professional qualifications, and sends it to the European Commission. (Quarterly)

3. adopts legislation to i) remove the prohibition to recognise the qualifications derived from franchised degrees to access or exercise an economic activity and to ii) ensure that holders of franchised degrees from other Member States have the right to work in Greece under the same conditions as holders of Greek degrees. (November 2012) 


\subsubsection{Services Directive: exploiting the information benefits of the Point of Single Contact}

The Government ensures:

1. that the Point of Single Contact (PSC) is fully operational in all sectors covered by the Services Directive. (December2012)

2. that the PSC distinguishes between procedures applicable to service providers established in Greece and those applicable to cross-border providers (in particular for the regulated professions). (December 2012)

3. that there is adequate connection between the PSC and other relevant authorities (including one-stop shops, professional associations and the recognition of professional qualifications) and that the submission of on-line applications as regards the recognition of professional qualifications is fully operational. (December 2012)

\section{Increasing the impact of structural and cohesion funds}

1. The Government meets targets for payment claims and major projects in the absorption of EU structural and cohesion funds set down in the table below. Compliance with the targets shall be measured by certified data. (Q4 2012)

Table 1: Targets for payment claims in the absorption of Structural and Cohesion Funds (programming period 2007-2013) to be submitted through 2013

(EUR million)

\begin{tabular}{l|l|l}
\hline & $\mathbf{2 0 1 2}$ & $\mathbf{2 0 1 3}$ \\
\hline $\begin{array}{l}\text { European Regional Development Fund } \\
\text { (ERDF) and Cohesion Fund }\end{array}$ & 2,850 & 3,000 \\
\hline European Social Fund (ESF) & 880 & 890 \\
\hline Target of first half of the year & 1,231 & 1,284 \\
\hline Total annual target & $\mathbf{3 , 7 3 0}$ & $\mathbf{3 , 8 9 0}$ \\
\hline
\end{tabular}

2. The Government submits complete applications for all major projects for which a Commission decision under Article 41 of Regulation (EC) No 1083/2006 has to be adopted. (Q4 2013)

3. In deciding the "Public Investment Budget" allocation, the Government ensures that the necessary national contribution remains available in order to complete the unfinished ERDF, ESF and Cohesion Fund projects of the 2000-2006 programming 
period and to cover the required national contribution including non-eligible expenditure under the Structural Funds and Cohesion Fund rules in the framework of the 2007-2013 programming period.

4. The Government continues to monitor on a quarterly basis the implementation of the priority projects, certain of which - as the functional review of the public administration, the "Elenxis" project for the tax control services, the land register, the solid waste management infrastructures, the railway projects, the e-prescription, the eprocurement, the development of a social economy sector and the national registry are critical for the development of the country. For priority projects in delay, a method for closer monitoring should be agreed (Q4 2012) in order to allow the adoption of the necessary measures on time. The priority projects should be completed by the end of 2015.

5. Support to SMEs managed at central and regional level is targeted to directly contribute to the development of the economy and the creation of sustainable employment, in particular for young people. Guidelines are issued for the definition of the conditions of granting state aid and its monitoring. (Q4 2012)

6. Within the review of the public procurement regulations (cfr. section 2.7.2.2.3), legislation is adopted, and immediately implemented, to shorten deadlines and simplify procedures on contract award. (Q1 2013)

7. The monitoring tool for expropriations is completed and operational and data are made accessible to the public. (Q1 2013)

8. Following the simplification initiative launched in May 2010 the Government takes measures to speed up absorption, accelerate payments to beneficiaries and simplify the management and project implementation on the basis of issues raised by the Commission and any other additional issues which may be proposed. In particular, the Government reviews the "sleeping" projects, un-activated delegations and sleeping contracts and informs the Commission on those eliminated. (Q4 2012) Similar exercises are periodically repeated until the expiry of the programming period.

9. The Government adopts measures and starts implementing an anti-fraud strategy in the field of the Structural Funds and the Cohesion Fund (Q2 2013). The Government establishes an efficient inter-service consultation procedure supported by an electronic system. For this, an integrated project will be set up and implemented with the exploitation of the existing infrastructure. (Q1 2013)

\section{Monitoring}

\subsection{Statistics}

High quality statistics are key for effective economic surveillance and policy design, and for the proper monitoring and execution of the budget. Significant progress has been made in improving the quality and coverage of statistics in Greece during the past two years. 
The Government commits to continue supporting the work of ELSTAT, and to honour its "Commitment on Confidence" signed by the Prime Minister of Greece on 29 February 2012 and which states among other things that "The Hellenic Republic ...makes the solemn commitments to fully respect the international and European standards for statistical data quality....to guarantee and defend the professional independence of the.... Hellenic Statistical Authority.... and to defend [it] against any efforts to undermine [its] credibility... and secure adequate and stable resources [for it]"

1. The Government, as stated in the aforementioned "Commitment on Confidence", further commits to decouple the budget of ELSTAT from that of the Ministry of Finance in line with the existing procedures and involving the Hellenic Parliament and the Court of Audit. From January $1^{\text {st }} 2013$ onwards, ELSTAT as a legal entity under Greek public law will get budgetary execution autonomy in accordance with the relevant legal framework, in particular Law 4072/2012, article 323. The Authorities commit to complete all actions needed to establish budgetary execution autonomy to ELSTAT in line with Law 4072/2012, article 323.

i. To achieve this, the Government amends the Law 3832/2010 regarding the non-applicability to ELSTAT of the provisions of article 25 of Law 2362/1995 and, more broadly, of any other provision the implementation of which is not consistent with the independence of ELSTAT (November, 2012).

2. Prior to disbursement, and to ensure a smooth and effective transition toward the new framework, the Authorities commit to clear all arrears toward ELSTAT for the financial years 2011 and 2012. Those arrears will be paid at the expense of ELSTAT's 2012 budget. 


\section{Annexes}

\subsection{Privatisation plan and intermediate steps}

Greece--Hellenic Asset Development Fund: Projects Under Development 2012-14

\begin{tabular}{|c|c|c|}
\hline \multicolumn{2}{|c|}{$\begin{array}{l}\text { Timing of Privatisation Project } \\
\text { (Launch of Tender) }\end{array}$} & Intermediate Steps \\
\hline \multicolumn{3}{|c|}{ I. State-owned enterprise/share sale } \\
\hline 2012 Q1 & Public Gas (DEPA) & Modification of statutory provision at time of privatisation. \\
\hline Q1 & Public Gas (DESFA) & State aid clearance (January 2013). \\
\hline Q4 & Football Prognostics Organization (OPAP) & $\begin{array}{l}\text { VLT regulation issuance and notification to EU (December 2012). Law on new tax provisions for } \\
\text { state aid clearance (December 2012). }\end{array}$ \\
\hline 2013 Q1 & Horserace Betting Organization (ODIE) & $\begin{array}{l}\text { Pending state aid clearance-, adopt legislation for the granting of the new license and the } \\
\text { subsequent liquidation of the company (January 2013). }\end{array}$ \\
\hline Q1 & Hellenic Post (ELTA) & $\begin{array}{l}\text { Ministerial decisions for (i) the determination of the content of universal service and (ii) the } \\
\text { compensation mechanism for USP drafted and prenotified to DGComp. }\end{array}$ \\
\hline Q1 & Hellenic Vehicle Industry (ELVO) & Transaction structure to be determined and agreed (February 2013). \\
\hline Q1 & Thessaloniki Water (EYATH) & $\begin{array}{l}\text { Establish regulatory framework (December 2012). Establish pricing policy and amend the } \\
\text { license (May 2013). }\end{array}$ \\
\hline Q1 & Mining and Metallurgical Company (LARCO) & Law for establishing a new company (January 2013). \\
\hline Q1 & Hellenic Defense Systems (EAS) & Identify EAS assets for privatisation (December 2012). \\
\hline Q2 & Hellenic Petroleum (HELPE) & Following divestment of DEPA. \\
\hline Q2 & Athens Water (EYDAP) & $\begin{array}{l}\text { Establish regulatory framework (December 2012). Establish pricing policy and amend license } \\
\text { (September 2013). Settlement of receivables from the State (September 2013). }\end{array}$ \\
\hline Q2 & Athens Airport (AIA) & Re-approach Hochtief Airports (December 2012). \\
\hline Q2 & Railways (Trainose) & $\begin{array}{l}\text { Remaining problems in Trainose will be resolved (February 2013). Trainose will then be } \\
\text { transferred to the HRADF (March 2013). }\end{array}$ \\
\hline Q3 & Public Power Corporation (PPC) & $\begin{array}{l}\text { MoEnergy issues the Energy Policy Objectives and a restructuring plan is fully identified } \\
\text { (December 2012). }\end{array}$ \\
\hline Pending cour & Casino Mont Parnes & $\begin{array}{l}\text { Pending legal action for the recovery of the state aid taken by the Ministry of Tourism } \\
\text { (December 2012) }\end{array}$ \\
\hline \multicolumn{3}{|c|}{ II. Concessions } \\
\hline n.a. & Hellenic Motorways & $\begin{array}{l}\text { Negotiations for the restart of projects currently in progress; ratification of reset agreement by } \\
\text { Parliament (December 2012). }\end{array}$ \\
\hline 2011 Q4 & State Lottery & Binding offers (December 2012). Submission to Court of Auditors (December 2012). \\
\hline 2013 Q1 & Egnatia Odos & $\begin{array}{l}\text { Launching of tender process dependent on: a) agreement/finalisation with Ministry of } \\
\text { Development on key characteristics of the concession and conclusion of business plan, b) } \\
\text { decision on tolling policy/toll collection system, c) treatment of Piraeus loan granted to } \\
\text { Egnatia Odos SA, and d) reset of the } 4 \text { Motorway concession projects }\end{array}$ \\
\hline Q1 & Small ports and marinas & Resolve issues related to urban zoning (December 2012). \\
\hline Q1 & Regional airports & National Airports Policy defined. Establish regulatory framework (January 2013). \\
\hline Q2 & Thessaloniki Port (OLTH) & State aid clearance (March 2013). Establish regula tory fra mework (April 2013). \\
\hline Q2 & Piraeus Port (OLP) & State aid clearance (March 2013). Establish regulatory framework (April 2013). \\
\hline Q2 & Large regional ports & State aid clearance (March 2013). Establish regulatory framework (April 2013). \\
\hline Q3 & South Kavala Gas Storage & Decision on the best exploitation option (December 2012). \\
\hline Q4 & Digital Dividend & Pass law to finalise licensing of TV stations and digital broadcasting (December 2012). \\
\hline n.a. & Mining rights & \\
\hline \multicolumn{3}{|c|}{ III. Real Estate } \\
\hline 2011 Q4 & Hellenikon 1 & $\begin{array}{l}\text { Transfer of Hellinikon SA ownership to HRADF (Pending decision: December 2012). Launch } \\
\text { Phase B of tender process (December 2012). }\end{array}$ \\
\hline 2012 Q1 & IBC & Get approval from Court of Audit (December 2012) and issue PD for ESCHADA (January 2013). \\
\hline Q1 & Cassiopi & Declassification of Naval outpost (December 2012). \\
\hline \multirow[t]{2}{*}{ Q1 } & Lot 1 (Afantou) & All intermediate steps have been fulfilled. \\
\hline & Sale/repo 28 buildings & All intermediate steps have been fulfilled. \\
\hline 2013 Q1 & Astir Vouliagmenis & $\begin{array}{l}\text { Negotiations ongoing with NBG. ESCHADA (Zoning and land permit) to be submitted (January } \\
\text { 2013). Process led by NBG. }\end{array}$ \\
\hline Q1 & Real Estate lot 2 & 40 properties to be identified (December 2012) and transferred to HRADF (March 2013). \\
\hline Q4 & Real Estate lot 3 & At least 1,000 real es tate properties to be transferred to HRADF (December 2013). \\
\hline
\end{tabular}

Source: HRADF 


\subsection{Regulated professions}

\subsubsection{List no. 1: list of restrictions on selected regulated professions to be repealed prior to the next disbursement}

1. Stevedores for land operations and at ports: simplify declaration procedures, repeal fixed fees for loading and unloading services and allow stevedores to be employed under private sector law.

2. Sworn-in valuers: Issue new legislation to eliminate the applicable fees, the system of numerus clausus, the nationality requirement, allow legal entities to pursue the profession and open up the areas reserved in exclusivity to this profession.

3. Accountants and tax consultants: clarify in circular 26801/DIOE 654 of 13 June 2012 that the professional identity card will be issued automatically within the three months period;; ii) clarify that it is SAEP the body responsible for the recognition of professional qualifications; iii) amend Ministerial Decision POL 1166/2011 to abolish the certification procedure provided for thereof, which is applicable to legal entities and natural persons; iv) include in the text of the circular references to the legal text of accountants/ tax advisors that have been abolished by law 3919/2011. In addition, amend sector specific legislation to eliminate inconsistencies with the 2011 law on regulated professions.

4. Temporary employment companies (TEC): amend sector specific law to i) reduce the minimum capital of $€ 176.083$ (Art.123.1 of law 4052/2012); ii) two eliminate / reduce the amounts of bank guarantees (Art. 126); iii) to lift the requirement of having a minimum number of employees; iii) extend the scope of temporary employment agencies to cover, among others, the provision of consulting and training services.

5. Private labour consultancy offices (PLCO): amend sector specific law to i) allow employers of PLCOs -other than its director, to undertake mediation; ii) lift requirements on built in infrastructure and on technical equipment; iii) lift requirement provided for in Art. 104.2 of law 4052/2012, whereby if the registered office of the PEA or its branch has changed, the procedure regarding start of PEA activity /occupation under Art. 101 of the same law shall apply; iv) allow PEAs to do activities other than intermediation in the premises where the PEA operates.

6. Real estate brokers: Amend sector specific law to drop the probationary period before an applicant can become a real estate broker (Art. 199 of law 4072/2012).

7. Actuaries: review the regulatory framework governing the examination process so as to prevent the Hellenic Actuarial Society (HAS) from determining indirectly the number of successful candidates in the examinations in the interest of the incumbents (cfr. the Hellenic Competition Commission's opinion no. 14/VI/2012).

8. Tourist guides: provide that the professional ID card will be issued within the three months period or earlier) and amend sector specific law to open the profession to holders of related university degrees. 
9. Energy inspectors: amend sector specific law to repeal minimum fees for energy inspection services and to simplify licensing.

10. Private providers of primary care services: amend sector specific law to eliminate inconsistencies with Law 3919/2011 law on professions.

11. Customs brokers: $\underline{c f r}$. section on trade facilitation.

12. Kiosks and cantinas in public buildings: remove restrictions for licenses in favour of specific groups.

13. Tourist offices: eliminate prior authorization scheme, minimum office space requirements, applicable quasi-fiscal charges and reduce the amount of bank guarantees or require touristy offices to get insurance.

14. Teaching at private tuition establishments and foreign language schools and teaching at home: remove the need for prior licence and replace it by a notification of commencement of activity. Remove the need to renew the licence every year.

15. Private primary and secondary schools: abolish the nationality requirement to get an establishment licence, amend the requirement for formal qualifications to the founder of a private school; abolish shareholding capital requirements, the ban on more than one establishment, minimum distances, the need to renew licenses annually; remove prohibitions to licensing to relatives of private primary and secondary schools license holders.

16. Private tuition establishment and foreign language schools: Amend the requirement for formal qualifications to the founder of a private tuition establishment and foreign language schools and remove prohibitions to licensing to relatives of license holders.

17. Vocational training institutes (VTI): Allow the advertising of VTI without the approval of the E.O.P.P.E.P.

18. Vocational training centres (VTC): Amend sector specific legislation to base the accreditation of a VTC on the basis of an integrity check of objective statutory requirements; ii) to allow private individuals to establish legal entities; iii) to renew the accreditation in the form of notification under Art. 3 of Law 3919/2011, by uncoupling it from the scoring system.

19. Post-secondary education centres: repeal the prohibition that post-secondary education centres cannot be established by shareholders or partners of the legal entity which applied for an establishment and operating licence; to renew operating licences in the form of notification under Art. 3 of Law 3919/2011; allow post-secondary education centres to recruit off-register staff notified to the supervising authority, as in the case of VTC; repeal the need to submit a letter of guarantee for the opening of a post-secondary education centre.

20. Accredited study centres: Amend the regulation on renewing licenses, so that licenses renewals are limited to the notifications under Art. 3 of law 3919/2011.

21. Press distribution agencies: remove prior authorization scheme and expand the number of economic activities carried out. 


\subsubsection{List no. 2: regulated professions / economic activities whose regulatory framework needs to be adjusted to applicable opinions of the Hellenic Competition Commission}

1. Professions under the Greek Ministry of Citizen Protection: i) sale of revolvers, pistols and target-shooting weapons; ii) preparation of explosives; manufacture, conversion, assembly, finishing and repair of firearms, and charging/recharging of firearm cartridges; iii) charging cartridges for hunting weapons for sale; iv) sale of fireworks and flare launchers; v) manufacture of fireworks and flare launchers; vi) operation of private security firms; vii) work by security staff of private security firms; viii) operation of private investigation offices; ix) work by staff of private investigation office: Cfr. HCC Opinion no. 13/VI/2012.

2. Dealers in antiques and more recent artifacts and restorers of fine art and antiquities: Cfr. HCC Opinion no. 18/VI/2012.

3. Professions / economic activities under the Greek Ministry of Citizen Protection harbour guard: i) Organisations certifying divers; ii) Suppliers of recreational diving services; iii) Rental of means of marine recreation; iv) Service boat operations; v) Towing operations; vi) Licensing for outdoor trading (stands or itinerant) on board ships; vi) Operation of casinos on board passenger vessels flying the Greek flag on international voyages; vii) Lifeguards: (i) Operation of public health establishments on anchored or floating craft and (ii) Operation of public health establishments on seafaring vessels: Cfr. HCC Opinion no. 22/VII/2012.

4. Professions / economic activities under the Ministry of Health and Social Solidarity, Directorate-General for Welfare: i) Care units for old people; ii) Day care centres for old people; iii) Creative activity centres for children; iv) Creative activity centres for children with disabilities; v) Nurseries and kindergartens; vi) Private childrens' camps; vii) Child welfare institutes: Cfr. HCC Opinion no. 25/VII/2012.

5. Petrol sellers, shotfirers, blasters and natural gas sales. Cfr. HCC Opinion no 26/VII/2012

\subsection{Agreed roadmap between the Greek Ministry of Justice and the EC/IMF/ECB for the review of the code of Civil Procedure}

1. By end-October 2012, the Taskforce on the review of the Code of Civil Procedure ("the Taskforce") prepares a briefing paper for its foreign expert members appointed by other EU Member States to assist its work. The objective of the briefing paper is to enable these foreign expert members to fully participate in its work, including developing alternative legal solutions to the identified reform needs based on crosscountry experience in other Member States.

2. Starting from October $\mathbf{2 0 1 2}$, the Taskforce also provides monthly updates by the end of each month on progress towards preparation by end-March 2013 of a detailed paper outlining the main proposals for amendments to the Code of Civil Procedure in line with the objectives defined in previous versions of this Memorandum. The detailed paper is presented to the EC/IMF/ECB shortly after its completion. 
3. By end-March 2013, the Taskforce prepares a detailed paper outlining the main proposals for amendments to the Code of Civil Procedure in line with the objectives set out in previous versions of this Memorandum.

4. By end-May 2013, the Government holds a series of workshops to discuss the findings and proposals in the detailed paper prepared by the Taskforce. These workshops will allow for broad consultation with domestic stakeholders and participation from recognised international experts in the field of civil procedure. On the basis of the outcome of this consultation, the Taskforce presents a revised version of the detailed paper to the EC/IMF/ECB and submits the revised paper, after reflecting comments from the $E C / I M F / E C B$, to the Government.

5. By end-June 2013, the Taskforce presents a detailed work plan, which provides for the preparation of the draft law by end-January 2014 and contains specific deadlines and deliverables for the chapters of the draft law, and implements the plan with immediate effect. At the end of each month the Ministry of Justice, Transparency and Human Rights assesses compliance with the detailed work plan and takes immediate remedial actions in case of anticipated or actual deviations.

6. Starting from July 2013, the Taskforce prepares the draft law on the basis of the above-mentioned detailed paper and work plan, and provides monthly updates by the end of each month on progress towards preparation by end-January 2014 of the draft law. The monthly updates describe the outcomes of the assessments of compliance with the detailed work plan and, if necessary, any remedial actions taken. The Ministry of Justice, Transparency and Human Rights holds at least three EC/IMF/ECB expert meetings throughout the drafting process: July 2013, October 2013 and January 2014. The meetings will allow for expert input in the drafting process and feedback on preliminary drafts of the legislation under preparation.

7. The Government finalises the study on the costs of civil litigation, its recent increase and its effects on workload of civil courts, together with recommendations, ensures that the recommendations are reflected in the draft law prepared by the Taskforce on the review of the Code of Civil Procedure and publishes the study. (December-2013)

8. The Government carries out a public consultation on the draft law bringing the Code of Civil Procedure in line with international best practice and submits the draft law to the Greek Parliament. (March-2014)

\subsection{Statistics to be published by the Ministry of Justice or Ministry of Finance}

(a) by end-October 2012, for each administrative tribunal, court of appeal and the supreme administrative court:

(i) the number of judges and administrative staff, with a breakdown for judges working in tax chambers or dealing primarily with tax cases;

(ii) the number of all cases;

(iii) the number of cases carried over from 2011; 
(iv) the number of cases filed in the first two quarters of 2012;

(v) the number of tax cases, with a breakdown according to case value (up to EUR 150,000, between EUR 150,001 to EUR 300,000 and above EUR 300,000);

(v) the number of tax cases carried over from 2011;

(vi) the number of tax cases filed in the first two quarters of 2012;

(vii) the recovery rate for all tax cases, which for the purposes of the MoU, shall mean the ratio of the amount collected by the creditor in enforcement proceedings following the issuance of an enforceable title - to the amount adjudicated by the court.

(b) by end-October 2012, for each civil court, court of appeal and the supreme civil court:

(i) the number of judges and administrative staff;

(ii) the number of all cases;

(iii) the number of cases carried over from 2011;

(iv) the number of cases filed in the first two quarters of 2012;

(v) the number of dormant cases on 30 June 2012, i.e. cases brought before the civil courts in which the relevant court's file records that they have been postponed or never received a hearing date and no party activity for receiving a hearing date has taken place for at least 18 months.

(c) by end-October 2012, at the first instance, court of appeal level and the supreme civil court:

(i) the number of all corporate insolvency cases;

(ii) the number of corporate insolvency cases carried over from 2011;

(iii) the number of corporate insolvency cases filed in the first two quarters of 2012

(iv) the average duration of corporate insolvency cases;

(v) a break-down of insolvency cases by sector (e.g., agriculture, construction, manufacturing, and services).

(d) by end-December 2012, quarterly updates of the information in (a) to (c) above relating to the previous quarter.

(iii) the number of corporate insolvency cases filed in the first two quarters of 2012;

(iv) the average duration of corporate insolvency cases; 
(v) the recovery rate for all corporate insolvency cases, which for the purposes of the MoU, shall mean the ratio of the amount collected by all creditors in reorganisation, debt enforcement or liquidation proceedings to the total amount owed by the company;

(vi) a break-down of insolvency cases by sector (e.g., agriculture, construction, manufacturing, and services).

(d) by end-December 2012, quarterly updates of the information in (a) to (c) above relating to the previous quarter.

\subsection{Non-exhaustive list of regulations on port work for review under the new port strategy}

- Port of Piraeus - law 1559/1950 (Gov. Gazette A 252/1950), law 2688/1999 (Gov. Gazette A 40/1.3.1999), Joint Ministerial Decision 5115.01/02/2004 on the approval of the General Regulation for Personnel (Gov. Gazette B 390/26.2.2004).

- Port of Thessaloniki - legislative decree 449/1970 (Gov. Gazette A 51/27.2.1970), law 2688/1999 (Gov. Gazette A 40/1.3.1999), Joint Ministerial Decision $5115.01 / 05 / 2003$ on the approval of the General Regulation for Personnel (Gov. Gazette B 1203/26.8.2003).

- Work Regulation of the Dockworkers of the Piraeus Port approved by Joint Ministerial Decision 45058/7/1971-Gov. Gazette B 579/22.7.1971)

- Joint Ministerial Decision 44885/8919/1956, as modified by Joint Ministerial Decision 117756/8295/1967-Gov. Gazette B 9/11.1.1967)

- Law 3239/1955

- Law 5167/1932

- Ministerial Decision F 10221/26816/929- Gov. Gazette B 2778/2.12.2011

\subsection{Additional fiscal measures for 2012 and Medium-Term Fiscal Strategy 2013-16}

The measures included in the medium-term fiscal strategy (MTFS) through 2016 are the following:

1. Rationalisations in wage bill by at least EUR 1,110 million in 2013 , and additional EUR 259 million in 2014 through:

- further reduction by 10 percent of fixed term contracts;

- the rationalization of the State wage bill (including reduction the wage bill for consultant doctors, and an hiring freeze at the Ministry of Citizen protection and at the Ministry of Education, Religious Affairs, Culture and Sport);

- progressive cuts in the monthly wages of employees under special wage regimes (judges, diplomats, doctors, professors, armed forces and police, airport personnel, and general 
secretaries) with effect by August 1, 2012, with the following marginal reduction schedule: 2 percent below EUR 1000; 10 percent for EUR 1000-1500; 20 percent for EUR 1500-2500; 30 percent for EUR 2500-4000; and 35 percent above EUR 4000)

- elimination of automatic wage promotions for the armed forces by 2014 yielding yearly at least EUR 88 million net of taxes and contributions

- elimination of seasonal bonuses of employees at the state and local governments, and at legal entities of public and private law,

- application of the uniform wage grid for public servants to the parliamentary staff (measure to be completed by December 2012),

- abolition of exceptions from the public sector wage grid reform introduced in 2011,

- suspension throughout 2016 of fiscal and performance bonuses for public sector employees,

- reduction in the local government wage bill,

- reductions in Members of Parliament compensations and other allowances

- reductions in cost for wages related to non-permanent secondary level teachers

- reduction in non-permanent teachers in universities and technical colleges

- reduction of intakes into professional academies,

- extension of the 1:5 hiring rule (according to which 1 employees could only be hired against 5 retirees) for the General Government through 2016

- placing 2,000 civil service employees into the mobility and exit scheme, by abolishing positions of specialized employees, addressing disciplinary cases (including via outright dismissals) and by abolishing positions associated with closed entities.

2. Savings in pensions by at least EUR 4800 million in 2013, and additional EUR 423 million in 2014, through

- the anticipation of the full effects of the pension reform in 2013,

- the increase of the general pensionable age from 65 to 67 and of all age limits for particular types of pensions while maintaining the current number of contributions required to qualify for the full pension at 40 years,

- cuts in new lump-sum benefits for public employees and for all Social Security Funds,

- the reduction of the overall monthly pension incomes (main and supplementary pensions) per pensioner (between $€ 1000$ - $€ 1500$ by 5 percent; $€ 1500$ - $€ 2000$ by 10 percent; $€ 2000$ $€ 3000$ by 15 percent and above $€ 3000$ by 20 percent), 
- cuts in pensions for special wage regimes equivalent to the cuts to wages in special wage regimes,

- cuts in pensions of military and police due to elimination of automatic wage promotions,

- increase in healthcare contributions for farmers

- elimination of seasonal bonuses for main and supplementary pensions (exceptions allowed for people with disabilities),

- means-testing pensions for specific categories of beneficiaries,

- elimination of special pension benefits of trade unionists and cross-checks to abolish ineligible pension benefits in 2013,

- reduction of pensions of elected staff.

3. Cuts in the state's operational expenditures by at least EUR 239 million in 2013 and additional EUR 285 million in 2014, through the gradual implementation of e-procurement for all public administration, 25 percent reduction in discretionary non-wage spending, reduction in subsidies to internal ferry boats and in grants to entities outside the general governments, elimination of grants to farmers' trade unions linked to assistance in applying for EU financial aid and tightening spending rule for the Green Fund in 2014 (at 2.5 percent of the stock of deposits).

4. Cost reductions and efficiency improvements in education expenditure by at least EUR 86 million in 2013, and additional EUR 37 million in 2014, through reduction in funding for entities outside of the General Government budget for education, rationalisation of the network of higher education institutions and reduction in expenses for central and regional education administrations..

5. Savings in state-owned enterprises by at least EUR 249 million in 2013 and additional EUR 123 million in 2014, through increase in revenue, reduction in transfers from the State budget to entities outside the General Government, operational and personnel expenses (harmonization of the wage grid of all state-owned enterprises in Chapter A with the uniform wage grid for state employees reducing average wages to no more than $€ 1900$ per month). In order to enforce the achievement of those savings, a new institutional framework is introduced by 2013 fixing the fiscal target for the overall sector in line with the MTFS, bettering the current monitoring system and introducing an enforcement mechanism in case of deviations from the target.

6. Cuts in operational defense-related expenditure producing savings by at least EUR 303 million in 2013 and additional EUR 100 million in 2014 through reduction in expenditures for military procurement by EUR 340 million in 2013-14, reduction in operational expenditures, closure of military camps and reduction of intakes into military academies.

7. Savings in healthcare and pharmaceutical expenditure by at least EUR 455 million in 2013, and additional EUR 620 million in 2014, through further development of the set of incentives and obligations for all participants along the medicines supply chain to promote the use of 
generic medicines, revises the co-payment structure for medicines to exempt from copayment only a restricted number of medicines related to specific therapeutic treatments, the revision of medicines' price, based on the three EU countries with the lowest prices, application of automatic claw-back mechanism to pharmaceutical producers which guarantees that the outpatient pharmaceutical expenditure does not exceed the targets of EUR 2440 billion in 2013 and EUR 2000 million in 2014 to be in line with the overall target of 1 percent of GDP by 2014, while maintaining supplies for patients. Increase in co-payments in hospitals and for prescriptions of drugs from 2014 onwards, reductions in hospitals' expenditures and a more effective implementation of the Health Map will also contribute to the expenditure reductions.

8. Savings from rationalisation of social benefits by at least EUR 217 million in 2013 and additional EUR 78 million in 2014, through

- the introduction of a length of residence in Greece criterion for the provision of pension benefits for uninsured individuals aiming to achieve savings (net of income taxes and social security contributions) of $€ 13$ million in 2013 and additional $€ 13$ million in 2014,

- the replacement of existing family benefits with a single targeted benefit which absorbs the tax family allowances,

- rationalization of the transportation reimbursement scheme for selected categories of patients in order to avoid frauds and misuse of public funds maintaining the service according to actual needs of patients,

- reduction of unemployment benefits targeted to specific geographical areas,

- targeting assistance pensions provided by EKAS to persons above 64 years,

- reduction of benefits to farmers

- introduction of two new social programmes capped, respectively, at 35 and 20 million from 2014 onwards: a Minimum Income Guarantee scheme applied in two pilot areas of the country with different socioeconomic profiles, and introducing a benefit equal to $€ 200$ per month payable for up to 12 months to long-term unemployed who exhaust the full length of unemployment benefit (12 months), provided they do not qualify for other training schemes and have family taxable income up to $€ 10,000$.

9. Cuts in state transfers to local governments by at least EUR 50 million in 2013 and additional EUR 160 million in 2014, through cuts in transfers from the State for ordinary expenses and investment of local government. In order to enforce the achievement of those savings, an internal stability pact is strengthened by 2013 fixing balanced budget targets, putting in place an effective system of monitoring municipalities' expenses, economic disincentives in case of deviations from the targets and excluding any possibility for financing deficits.

10. Cuts in expenditure by the public investment budget (domestically-financed public investment, and investment-related grants) by EUR 150 million in 2013 and additional 150 million in 2014. 
11. Increases in revenue by at least EUR 1689 million in 2013 and additional EUR 1799 million in 2014 , through increases in the fee for law suits, an increase of tax on ship owners' activities, reducing VAT refunds for farmers, reduction of diesel excise duty subsidy provided to farmers, equalization of the excise tax on LPG and motor diesel oil by raising the LPG tax, equalization of social security contributions by raising the ceiling for employees first employed before 1993 to that of employees first employed after 1993, a reform of tobacco excise taxation, imposing a 30 percent taxation on OPAP's gross gaming revenue, equalization of taxation of winnings towards 10 percent rate without deductibles, and reintroduction of the 2011 one-off taxation of pools, yachts and luxury goods from 2014 onwards. By December 2012 a tax reform will be adopted that aims at simplifying the tax system, means-testing family allowances in order to better targeting benefits according to real needs, eliminating selected income tax credits and deductions and preferential regimes, thus broadening bases and ensuring an increase in revenue by about EUR 1.8 billion.

If necessary, after consultation with the European Commission, ECB and IMF staff, these measures may be replaced with other measures yielding comparable or higher savings.

\subsection{Provision of data}

During the programme, the following data shall be made available to the European Commission, the ECB and the IMF staff on a regular basis.

These data should be sent to the following e-mail address:

\section{ecfin-greece-data@ec.europa.eu}

This address should also be used for the transmission of other data and reports related to the monitoring of the programme. 
To be provided by the Ministry of Finance

\begin{tabular}{|c|c|}
\hline $\begin{array}{l}\text { Preliminary monthly data on the state } \\
\text { budget execution (including } \\
\text { breakdown by main categories of } \\
\text { revenue and expenditure and by line } \\
\text { ministry). } \\
\text { (Data compiled by the Ministry of } \\
\text { Finance) }\end{array}$ & $\begin{array}{l}\text { Monthly, } 15 \text { days after the end } \\
\text { of each month; these data } \\
\text { should also be included in } \\
\text { subsequent transmissions in } \\
\text { case of revision. }\end{array}$ \\
\hline $\begin{array}{l}\text { Updated monthly plans for the state } \\
\text { budget execution for the remainder } \\
\text { of the year, including breakdown by } \\
\text { main categories of revenue and } \\
\text { expenditure and by line ministry. } \\
\text { (Data compiled by the Ministry of } \\
\text { Finance) }\end{array}$ & $\begin{array}{l}\text { Monthly, } 30 \text { days after the end } \\
\text { of each month. }\end{array}$ \\
\hline $\begin{array}{l}\text { Monthly data on the public wage bill } \\
\text { (of general government, including a } \\
\text { breakdown in nominal wage and } \\
\text { allowances paid to government } \\
\text { employees per line ministry and } \\
\text { public entity), number of employees } \\
\text { (including a breakdown per ministry } \\
\text { and public entities outside the central } \\
\text { government) and average wage } \\
\text { (including the relative shares of the } \\
\text { base wage, allowances and bonuses). } \\
\text { (Data compiled by the Ministries of } \\
\text { Interior and Finance) }\end{array}$ & $\begin{array}{l}\text { Monthly, } 30 \text { days after the end } \\
\text { of each month. }\end{array}$ \\
\hline $\begin{array}{l}\text { Preliminary monthly cash data on } \\
\text { general government entities other } \\
\text { than the state. } \\
\text { (Data compiled by the Ministry of } \\
\text { Finance) }\end{array}$ & $\begin{array}{l}\text { Monthly, } 30 \text { days after the end } \\
\text { of each month, these data } \\
\text { should also be included in } \\
\text { subsequent transmissions in } \\
\text { case of revision. }\end{array}$ \\
\hline
\end{tabular}


To be provided by the Ministry of Finance (continued)

\begin{tabular}{|c|c|}
\hline $\begin{array}{l}\text { Monthly data on staff: number of } \\
\text { employees, entries, exits, transfers } \\
\text { among government entities; and } \\
\text { from and into the labour reserve, per } \\
\text { entity. } \\
\text { (Data compiled by the Ministries of } \\
\text { Interior and Finance) }\end{array}$ & $\begin{array}{l}\text { Monthly, } 30 \text { days after the end of } \\
\text { each month. }\end{array}$ \\
\hline $\begin{array}{l}\text { Weekly information on the } \\
\text { Government's cash position with } \\
\text { indication of sources and uses as well } \\
\text { of number of days covered. } \\
\text { (Data compiled by the Ministry of } \\
\text { Finance) }\end{array}$ & $\begin{array}{l}\text { Weekly on Friday, reporting on } \\
\text { the previous Thursday. }\end{array}$ \\
\hline $\begin{array}{l}\text { Data on below-the-line financing for } \\
\text { the general government. } \\
\text { (Data compiled by the Ministry of } \\
\text { Finance) }\end{array}$ & $\begin{array}{l}\text { Monthly, no later than } 15 \text { days } \\
\text { after the end of each month; } \\
\text { these data should also be } \\
\text { included in subsequent } \\
\text { transmissions in case of revision. }\end{array}$ \\
\hline $\begin{array}{l}\text { Data on expenditure pending } \\
\text { payment (including arrears) of the } \\
\text { general government, including the } \\
\text { State, local government, social } \\
\text { security, hospitals and legal entities. } \\
\text { (Data compiled by the Ministry of } \\
\text { Finance on the basis of basic data } \\
\text { from the several line ministries) }\end{array}$ & $\begin{array}{l}\text { Quarterly, within } 55 \text { days after } \\
\text { the end of each quarter. }\end{array}$ \\
\hline $\begin{array}{l}\text { Data on use of international } \\
\text { assistance loans split among } \\
\text { following categories: Financial } \\
\text { stability fund, segregated account, } \\
\text { debt redemption, interest payments, } \\
\text { other fiscal needs, building of cash } \\
\text { buffer; per quarter and cumulative }\end{array}$ & $\begin{array}{l}\text { Quarterly, by the end of each } \\
\text { quarter. }\end{array}$ \\
\hline
\end{tabular}




\section{To be provided by the Ministry of Finance (continued)}

Data on public debt and new guarantees issued by the general government to public enterprises and the private sector.

Data on maturing debt (planned redemptions per month, split between short-term (Treasury bills and other short-term debt) and long-term (bonds and other longterm) debt).

Data on planned monthly interest outflows.

(Data compiled by the Ministry of Finance)

Data on assets privatised and proceeds collected.

(Data compiled by the Ministry of Finance)

Data on state-owned enterprises: revenue, costs, payroll, number of employees and liabilities (including maturities of public enterprises' debts)

(Data compiled by the Ministry of Finance)

Monthly statement of the transactions through off-budget accounts.

(Data compiled by the Ministries of Finance and Education, Religious Affairs, Culture and Sport)
Monthly, within one month.

Monthly.

Monthly, within three weeks of the end of each month for the ten largest enterprises. Quarterly within three weeks of the end of each quarter for the other enterprises.

Quarterly for the maturities of state-owned enterprises' liabilities.

Monthly, at the end of each month. 
To be provided by the Ministry of Finance (continued)

\begin{tabular}{l|l}
\hline $\begin{array}{l}\text { Monthly statement of the operations on the } \\
\text { special accounts. }\end{array}$ & $\begin{array}{l}\text { Monthly, at the end of } \\
\text { each month. }\end{array}$ \\
$\begin{array}{l}\text { (Data compiled by the Ministry of Finance) } \\
\text { Report on progress with fulfilment of policy } \\
\text { conditionality. }\end{array}$ & $\begin{array}{l}\text { Quarterly before the } \\
\text { respective review starts. }\end{array}$ \\
$\begin{array}{l}\text { (Report prepared by the Ministry of Finance) } \\
\text { Monthly data on health care expenditure by } \\
\text { the social security funds with a lag of three } \\
\text { weeks after the end of the respective } \\
\text { quarter. }\end{array}$ & $\begin{array}{l}\text { Monthly, within three } \\
\text { weeks of the end of each } \\
\text { month. }\end{array}$ \\
$\begin{array}{l}\text { (Data compiled by the Ministries of Labour } \\
\text { and Health) }\end{array}$ & \\
\hline
\end{tabular}


To be provided by the Bank of Greece

\begin{tabular}{l|l}
\hline $\begin{array}{l}\text { Assets and liabilities of the Bank of } \\
\text { Greece. }\end{array}$ & $\begin{array}{l}\text { Weekly, next working } \\
\text { day. }\end{array}$ \\
\hline $\begin{array}{l}\text { Assets and liabilities of the Greek } \\
\text { banking system - aggregate } \\
\text { monetary balance sheet of credit } \\
\text { institutions. }\end{array}$ & $\begin{array}{l}\text { Monthly, 30 days after } \\
\text { the end of each month. }\end{array}$ \\
\hline $\begin{array}{l}\text { Evolution of the external funding } \\
\text { provided by Greek banks to their } \\
\text { subsidiaries abroad. }\end{array}$ & $\begin{array}{l}\text { Monthly, 15 days after } \\
\text { the end of each month. }\end{array}$ \\
\hline $\begin{array}{l}\text { Report on banking sector liquidity } \\
\text { situation. }\end{array}$ & $\begin{array}{l}\text { Weekly, next working } \\
\text { day. }\end{array}$ \\
\hline $\begin{array}{l}\text { Report on the evolution of financial } \\
\text { stability indicators. }\end{array}$ & $\begin{array}{l}\text { Quarterly, 30 days after } \\
\text { the publication data of } \\
\text { each quarter. }\end{array}$ \\
\hline $\begin{array}{l}\text { Report on results from the regular } \\
\text { quarterly solvency assessment } \\
\text { exercise. }\end{array}$ & $\begin{array}{l}\text { Quarterly, 15 days after } \\
\text { the end of each quarter } \\
\text { depending on data } \\
\text { availability. }\end{array}$ \\
\hline $\begin{array}{l}\text { Weighted average of Loan-to-value } \\
\text { estate collateral }\end{array}$ & Yearly. \\
\hline
\end{tabular}

To be provided by the Hellenic Financial Stability Fund

Detailed report on the balance sheet of the Hellenic Financial Stability Fund with indication and explanation of changes in the accounts. 


\section{Abbreviations}

\begin{tabular}{|c|c|}
\hline ASEP & Supreme Council for Staff Selection \\
\hline $\mathrm{CPB}$ & Central Purchasing Bodies \\
\hline DEPA & Public Gas Corporation \\
\hline DRG & Diagnostic-Related Group \\
\hline DSO & Distribution System Operator \\
\hline EC & European Commission \\
\hline $\mathrm{ECB}$ & European Central Bank \\
\hline EEA & European Economic Area \\
\hline EETT & Hellenic Telecommunications and Post Commission \\
\hline EFSF & European Financial Stability Facility \\
\hline EKEVYL & National Centre for Medical Technology \\
\hline ELSTAT & Hellenic Statistical Authority \\
\hline EOF & National Organisation for Medicines \\
\hline EOPYY & National Organisation for the provision of Health services \\
\hline EPY & Health Procurement Commission \\
\hline ERDF & European Regional Development Fund \\
\hline ESA & European System of Accounts \\
\hline ESF & European Social Fund \\
\hline ESY & National Health System \\
\hline EU & European Union \\
\hline GDP & Gross Domestic Product \\
\hline GEMI & General Commercial Registry \\
\hline HRADF & Hellenic Republic Asset Development Fund \\
\hline IDIKA & E-governance of social insurance \\
\hline IMF & International Monetary Fund \\
\hline KTEL & Joint Fund for Bus Receipts \\
\hline LNG & Liquefied Natural Gas \\
\hline LTV & Loan-to-value \\
\hline MEFP & Memorandum of Economic and Financial Policies \\
\hline MTFS & Medium-Term Fiscal Strategy \\
\hline NHS & National Health System \\
\hline OASA & Athens Urban Transport Organisation \\
\hline OECD & Organisation for Economic Cooperation and Development \\
\hline OGA & Agricultural Insurance Organisation \\
\hline OSE & Railway Organisation of Greece \\
\hline OTE & Hellenic Telecommunication Company \\
\hline PPC & Public Power Corporation \\
\hline PSC & Point of Single Contact \\
\hline RAE & Regulatory Authority for Energy \\
\hline RSPP & Radio Spectrum Policy Programme \\
\hline SPA & Single Payment Authority \\
\hline SPPA & Single Public Procurement Authority \\
\hline TAP & trans-Adriatic pipeline \\
\hline TEN-T & Trans European Transport network \\
\hline TSO & Transmission System Operator \\
\hline WHO & World Health Organisation \\
\hline
\end{tabular}




\title{
INTERNATIONAL MONETARY FUND
}

\section{GREECE}

\section{First and Second Reviews Under the Extended Arrangement Under the Extended Fund Facility, Request for Waiver of Applicability, Modification of Performance Criteria, and Rephasing of Access Supplementary Information}

\author{
Prepared by the European Department \\ (In Consultation with Other Departments) \\ Approved by Reza Moghadam and Lorenzo Giorgianni \\ January 15, 2013
}

1. This supplement provides information that has become available since the staff report for the first and second reviews under the extended arrangement was issued. The authorities have made further progress with program implementation, and while in a few areas there was some loss of momentum, the authorities have committed to catch up by the time of the next review. Thus the thrust of the staff appraisal remains broadly unchanged.

\section{Recent Economic Developments}

2. Recent developments point to continued adjustment against a recessionary backdrop, together with a continued recovery of bank deposits.

- The economy remains very weak. Industrial production dipped 3.5 percent year-onyear in October, in line with program assumptions, while the unemployment rate continued to climb, reaching almost 27 percent (seasonally adjusted).

- Disinflation is running slightly ahead of expectations. CPI inflation for 2012 was 1 percent (average) and 0.3 percent (end-period), versus staff projections of 1.2 and 1.3 percent, respectively. Meanwhile, core inflation was -0.1 percent (average) and -0.9 percent (year-end) in December.

- External adjustment continues apace. The October current account outcome was in line with staff projections. Data show continued sharp import contraction (down 11.7 percent in January-October, year-on-year) and positive but low growth of exports (up 0.3 percent).

- The recovery of deposits has gained significant momentum. Greek banks saw an inflow of $€ 4.9$ billion (3.3 percent) in private deposits in December, with a notable acceleration following the European partners' approval of the review. This marks the fourth consecutive month of net inflows. 


\section{Program Implementation}

3. Recent policy developments point to continued progress with implementation, but a few problems with full and timely observance of benchmarks. Since issuance of the staff report, information has become available about quantitative targets, remaining prior actions, structural benchmarks, other MEFP commitments for end-2012, and adherence to conditions for the January European loan tranche.

\section{Quantitative targets}

4. Available data confirm that one quarterly performance criterion for endDecember has been met, and one indicative target has been missed. The end-December performance criterion on external arrears was met. Data on the remaining end-December performance criteria are not yet available (the authorities' Letter of Intent requests the appropriate waivers of applicability), but we expect them to have been met. Preliminary data indicate that the indicative target of $€ 3.2$ billion for privatization revenues in 2012 was missed, with realized revenues of only $€ 6$ million. As discussed in the staff report, this reflects the standstill in privatization for several months around the elections, and the absence of the Privatization Fund board for an extended period. Data on the other indicative target on domestic arrears are not yet available.

\section{Prior actions}

5. The authorities were not able to complete every subcomponent of one fiscal prior action, but they took compensating actions. One element of the fiscal prior action mandated signing an $\mathrm{MoU}$ between the government and owners of the merchant fleet to ensure payment of tonnage tax aimed at raising $€ 80$ million in 2013 and $€ 140$ million each year in 2014-16. The MoU has not yet been signed. However, separate legislation was passed that imposes tonnage taxes, which is expected to yield €75-80 million per year starting in 2013. The authorities expect to sign the MoU with Greek ship owners after their annual meeting on February 8. This would raise a further $€ 60-65$ million per year starting in 2014, bringing the revenues from new shipping taxes to the total envisioned in the MEFP. Since the authorities also raised the withholding tax on deposits to 15 percent in 2013 (a year earlier than envisaged), staff does not foresee a fiscal gap from the delayed completion of this measure.

\section{Structural benchmarks}

6. Parliament passed a tax bill that broadens the tax base, simplifies the tax system, and contributes towards the fiscal adjustment effort. With passage of this bill, staff considers that the authorities have also met the overdue program structural benchmark on tax reform (scheduled for end-June 2012). As noted in the staff report, there will be a second stage of the tax reform in May 2013, which will focus largely on the tax code as well as further income tax simplification. It was agreed with the authorities that this legislation 
would also address requirements for taxpayers to collect receipts (an activity that delivers minimum benefits while imposing significant burdens), and residual issues in the taxation of income from real estate.

\section{The authorities do not appear to have met the three structural benchmarks for end-December under the program.}

- $\quad$ Meeting targets for key performance indicators for revenue administration. These indicators target improvements in the collection of tax debts and expansion of tax audits, among other things. Data is not yet available, but based on end-November outcomes staff considers it highly unlikely that the targets for end-December will be achieved. As noted in the staff report, lack of leadership and political commitment has taken a toll on reform efforts this year, and the authorities do not appear to have been able to catch up during the fourth quarter. The appointment on January 10 of a new head of the tax administration will address the leadership vacuum in the organization, and this can help set the stage for better performance in 2013. The authorities committed to set key performance indicators for Q1 2013 and achieve these before completion of the next review.

- $\quad$ Quarterly performance indicators for public financial management. The indicators cover the adoption of commitment registers across the general government, and improvements in data reporting from commitment registers. As of end-November, only 66 percent of entities were reporting, versus a target of 90 percent for end-year. Again, it is unlikely that this ground was made up in December. However, the implementation of General Directorates of Financial Services underway in all line ministries (to improve expenditure control) is one step to improve performance in 2013. The authorities committed to set key performance indicators for Q1 2013 and achieve these before completion of the next review.

- $\quad$ Screening and cleaning of existing legislation on a list of regulated professions. The authorities have taken actions to liberalize regulated professions (prior action), and as reported in the staff report, the vast majority of professions have now been addressed. However, the review of legislation for restrictions on remaining professions and economic activities is still underway. Draft legislation has been prepared that would partly implement this benchmark, and the authorities expect full implementation not later than end-March.

\section{Other structural reforms}

8. Financial sector restructuring continues to progress. The HFSF has provided bridge capital to raise core tier one ratios of the four core banks to 9 percent, using funds disbursed by Greece's European partners in December. The resolution of Hellenic Postbank via a purchase and assumption transaction (end-January structural benchmark) is expected to 
be completed next week. The four core banks (three after the pending merger between National Bank of Greece and Eurobank is completed) are integrating previous acquisitions and have not been able to make acceptable binding offers for Postbank. In the event that a $\mathrm{P} \& \mathrm{~A}$ transaction is not feasible, resolution of Postbank via a bridge bank structure may be necessary.

9. A number of other actions contained in the MEFP for end-December 2012 have been completed, although some actions are still pending. Completed actions include increases in end-user prices for low voltage electricity customers, with further increases expected in May and July that will complete the process of liberalization and cost-recovery; publication of a list of regional tax offices to be closed (to improve efficiency and governance through centralization); and the publication of the trade facilitation strategy. Incomplete actions include full staffing of the directorate of planning, management, and monitoring of reforms at the Office of the Prime Minister; and publication of a report on reducing the nontax case backlog in the courts (which was hampered by a "work to rule" strike by the judiciary). Delays in the actions will need to be overcome during the next review cycle to avoid affecting broader structural reform implementation, and in particular improvements in the business environment. Finally, several actions to support the privatization program have been delayed, although the authorities and staff agree that this need not have implications for the timing of the sales.

\section{European support}

10. The authorities have completed the actions ("milestones") required to unlock the $€ 2$ billion disbursement of the January tranche from Greece's European partners. The actions were passage of the tax bill passed on January 12 and the increase in electricity prices and the renewable energy levy.

\section{Supplementary Staff Assessment}

\section{Greece has made further progress with reforms, but timely program} implementation remains a constant challenge that will require intensive and uninterrupted efforts. On the one hand, the completion of all the prior actions (with one exception, where a substitute measure was found), and the completion of the tax reform benchmark and other end-year MEFP commitments, is evidence of the authorities' commitment to the reform agenda under the program. On the other hand, slippages in the December structural benchmarks point to the challenges ahead in program implementation. The tasks ahead and the obstacles to successful implementation are particularly notable in the area of tax administration. As noted in the staff appraisal, reform of the tax administration is critical to the program, and it will require the full mobilization of political support to succeed. 
January 16, 2013

\section{IMF Executive Board Completes First and Second Reviews Under Extended Fund Facility Arrangement for Greece and Approves €3.24 Billion Disbursement}

The Executive Board of the International Monetary Fund (IMF) today completed the first and second reviews of Greece's economic performance under a program supported by a four-year Extended Fund Facility (EFF) arrangement for Greece. The completion of the review enables the disbursement of an amount equivalent to SDR 2.798 billion (about $€ 3.24$ billion or US $\$ 4.3$ billion), bringing total Fund disbursements under the EFF arrangement to an amount equivalent to SDR 4.197 billion (about $€ 4.86$ billion or US\$6.46 billion). In completing the review, the Executive Board also approved waivers of applicability of end-December 2012 performance criteria, modified performance criteria, and rephased disbursements under the arrangement.

Following a political crisis that delayed implementation of the economic program, understandings were reached with the government on a fully recalibrated economic program to be supported under the EFF arrangement. Policies were modified to deal with stronger macroeconomic headwinds and to better reflect observed implementation capacity. The fiscal adjustment path was lengthened by two years to 2016 to give Greece more time to reach the primary balance target, privatization targets were adjusted downwards to reflect weak market conditions, and the authorities specified the adjustment measures necessary to close the fiscal gap through 2014. In addition, authorities took measures to liberalize product markets and advance bank recapitalization. The Greek government also reached understandings with its European partners on a revised financing framework, including steps to ease its debt burden.

The EFF arrangement, which was approved on March 15, 2012 (see Press Release No. $\underline{12 / 85}$ ), is part of a joint package of financing with euro area member states amounting to $€ 172$ billion over four years. It entails exceptional access to IMF resources, amounting to about 2,159 percent of Greece's quota.

Following the Executive Board's discussion, Ms. Christine Lagarde, IMF Managing Director and Executive Board Chair, said: 
"The program is moving in the right direction, with strong fiscal adjustment and notable labor-cost competitiveness gains. While the program has been adjusted to take account of the deeper recession and implementation capacity, the strategy remains focused on restoring growth, competitiveness, and debt sustainability. Forceful structural reforms and broad-based domestic support will be needed to meet challenges, alongside long-term support from Greece's European partners.

"Greece has made progress with structural reforms, reflected in recent actions to reduce nonwage labor costs and reform the product market. However, much more remains to be done to achieve the critical mass of reforms needed to boost productivity and lower prices. Ambitious reductions in barriers to competition are crucial. It will also be important for the government to deliver its privatization plans and to take appropriate steps to strengthen the governance of the process, if necessary.

"Efforts must continue to restructure and strengthen the banking system. With the finalization of the bank recapitalization framework, it is vital that the new monitoring and supervisory framework be made effective to protect the public interest and prevent state interference in management. Additional financing from euro area member states to allow Greece to redeem treasury bills from banks could support liquidity and credit creation.

"Greece's fiscal effort has been impressive by any measure. The frontloaded adjustment will help bring spending back towards pre-euro levels, and has been designed to protect the most vulnerable. Looking ahead, Greece needs to radically overhaul its tax administration to bolster tax collections, fight tax evasion, and shrink the public sector, in particular through targeted redundancies.

"Steps are being taken to put Greece's debt on a more sustainable path. Greece's European partners have extended repayment periods on their loans and provided assurances that they will consider additional conditional measures and assistance to reduce debt to substantially below 110 percent of GDP by 2022 .

"Euro area member states have committed to work together with the Greek authorities and the IMF to ensure the success of the program, reaffirmed the IMF's preferred creditor status, and committed to providing adequate support to Greece during the program and beyond, provided that Greece continues to cooperate closely with the IMF in the implementation of appropriate adjustment policies. This would facilitate a return to debt sustainability and timely repayments to the Fund." 


\section{Statement by Thanos Catsambas, Alternate Executive Director for Greece January 9, 2013}

Following a prolonged pre-election period and two general election rounds, a new government, representing a three-party coalition headed by Prime Minister Antonis Samaras, was sworn in on June 21, 2012. The new government, known as the Government of National Responsibility, focused on regaining the ground that had been lost as a result of the political uncertainty and initiated a series of measures aiming at accelerating the economic adjustment program that had been approved by the IMF Board last March. The policy initiatives cover a broad range of areas: fiscal policy, revenue administration, public financial management, privatization and state-owned land development, structural reforms in product, services and labor markets, restructuring and recapitalization of the banking system, and improved absorption of EU structural funds. In short, Greece has continued to push ahead with a wide array of fundamental reforms, which are set to transform and modernize its economy in an unprecedented way.

\section{Outcomes And Successes of Recent ECONOMIC Policies}

From its inception, the main objective of the IMF/EU/ECB-supported program has been to help Greece regain its lost competitiveness and set the stage for a new development model based on flexible labor and product markets, an efficient public sector, and an effective tax administration. As a result of the policies adopted since the beginning of the EFF-supported program, unit labor costs have continued their downward path that began in 2010-2011, and it is now estimated that at the end of 2012 Greece had regained more than 18 percentage points of the competitiveness lost in 2001-2009. The recovery in competitiveness and attendant increase in exports, together with the fall in imports due to the domestic recession, has significantly shrunk the current account deficit from a high of about 15.0 percent of GDP in 2008 to an estimated 4.2 percent for 2012, thanks also to a reduction in interest payments on government debt following the successful PSI in February 2012. 

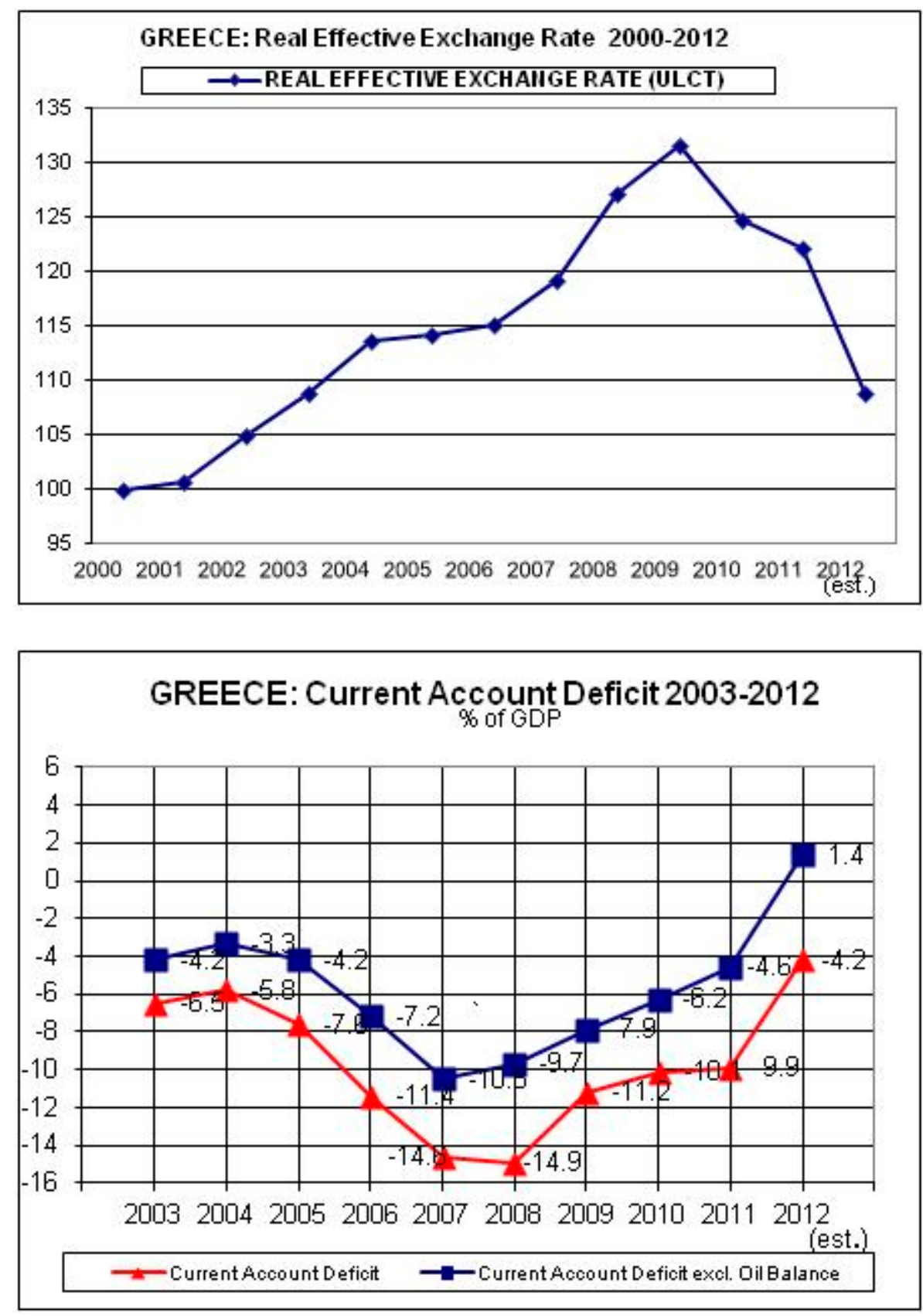

The significant achievements on the structural front since the beginning of the Fundsupported adjustment program have been reflected in a substantial improvement of the business environment; as a result, the 2013 Doing Business Report of the IFC has ranked Greece $78^{\text {th }}$, up from $89^{\text {th }}$ in the previous edition, and has listed the country as one of the top 10 economies that has shown the greatest progress across three or more areas. In the case of Greece notable improvements include (a) simplifying the construction permit process; (b) introducing legislation to protect investors, and (c) resolving insolvency issues. Also, in 2012 Greece ranked first according to the Adjustment Progress Indicator calculated by the Lisbon Council for the 17 Euro area countries, plus Poland, Sweden and the U.K. 


\section{The Political Economy of The Reform EfForts}

Over the last two-and-a half years Greece has achieved impressive macroeconomic adjustments, but one should be mindful that the effects in the real economy have been far reaching and painful: the cumulative decline of GDP in the five-year period 2008-2012 is expected to exceed 21 percent, and the rate of unemployment has tripled from 7.6 percent in 2008 to 23.6 percent in the second quarter of 2012 and to 26.0 percent in September. Youth unemployment reached 56.4 percent. Greece is beginning to face an "unemployment trap": the length of the Greek recession entails the risk that the skills of the long-term unemployed will become obsolete; this would hinder the re-entry of unemployed workers into the labor force.

In addition, the financing conditions for enterprises, in particular the availability of bank loans, have continued to deteriorate; the accumulation of arrears by the public sector and negative credit flows hinder export activity by Greek firms, including entrepreneurial initiatives to move from declining traditional sectors to dynamic export-oriented sectors. Moreover, the protracted recession has intensified uncertainty about the future of the Greek economy, which in turn has led to capital flight and has discouraged foreign investment and the mobilization of domestic capital formation.

Such developments, but particularly the high unemployment rate and the increase in the official poverty statistics, risk eroding the ownership of the adjustment program among significant strata of the population. Nevertheless, and despite the painful austerity measures, Greeks continue to envisage the future of the country within the Eurozone.

\section{THE WAY FORWARD}

The Greek government recognizes the importance of continuing structural reforms to achieve sustainable economic growth. Following the impressive achievements in the labor market, the main goal of the Fund-supported program going forward is to further liberalize the product and services markets by removing unnecessary restrictions and barriers to entry that currently impede competition and have been an important factor in oligopolistic pricing. Equally important, the Government plans to achieve the fiscal goals under the program not only through tax and expenditure measures, but also by a broad overhaul of Revenue Administration and Public Financial Management rules and procedures. Finally, the Government is taking decisive steps to accelerate the privatization program that effectively stalled during 2012.

Within this context, the Greek authorities welcome the extension of the fiscal consolidation period until 2016 and the consequent smoothing of the adjustment targets. They also appreciate the availability of additional financing through the generous easing of repayment terms and new commitments by the European partners. They are also pleased to note the success of the buyback initiative of Greek bonds that took place in December. The government is committed to take advantage of this extended framework by intensifying its efforts in all areas of macroeconomic policy and management, 
overcoming entrenched vested interests, and reinvigorating the ownership of the program by tackling tax evasion and corruption in public administration.

\section{Specific Policies and Measures}

\section{A. Fiscal Developments, the 2013 Budget and the Medium-term Fiscal Framework}

Since the adoption of the Stand-by arrangement in May 2010, a key emphasis of the program has been on achieving the fiscal consolidation targets; by the end of 2012 the general government primary deficit is estimated at 1.5 percent of GDP, down from 2.2 percent in 2011 and 10.4 percent in 2009; this cumulative adjustment of 8.9 percentage points within three years is an unprecedented performance among OECD countries. Given that the target in the adjustment program calls for a government primary surplus of 4.5 percent of GDP by 2016, the estimated 2012 deficit suggests that, already by the end of last year, Greece had already covered 60 percent of the distance, i.e. a cumulative 8.9 percentage points reduction out of 14.9 percentage points of GDP expected by 2016.

The achievement of the 2016 target is predicated on a series of new front-loaded revenue and expenditure measures amounting to $71 / 4$ percent of GDP, of which 5 percent of GDP are part of the 2013 Budget that has already been approved by parliament. The core of the measures affect the most sensitive areas of public spending: further consolidation in pensions, wages, salaries and social benefits, and substantial cuts in wasteful health and education spending, coupled with further consolidation in defense and public administration expenditures.

\section{B. Revenue Administration and Public Financial Management}

The Greek authorities recognize that policies may be inadequately implemented unless there is a parallel strengthening of the underlying administrative procedures. Ownership of the program by the Greek citizens is a fundamental ingredient for its success. In turn, ownership hinges on a sense of justice in the distribution of the burden of adjustment. To achieve this goal, far reaching changes have been set in motion to root out tax evasion and to ensure a fair allocation of expenditure cuts across ministries.

- In Revenue Administration, several improvements in the internal workings of the tax offices have been advanced, but two major initiatives stand out. First, the establishment of a new Secretary General of Public Revenues, with a five-year tenure, who will be overseeing the internal revenue service (tax and customs) with a great degree of autonomy. The Secretary General will have the authority, inter alia, to develop, update and supervise the tax administration's strategic planning; set goals and assessment criteria; appoint the heads of organizational units; and take measures to combat corruption. This reform is of utmost importance in order to improve the tax administration's effectiveness and enhance its independence from political pressure. Second, the pursuit of potential major tax evaders by cross checking bank deposits outside Greece with the corresponding tax returns of the 
owners in the past several years. If a major and inexplicable discrepancy is identified between the tax returns and the remittances abroad, the taxpayers will be liable to surcharges and penalties. It is the first time in the history of Greece that a systematic action against tax evasion is taken on such large scale.

- In Public Financial Management several continuing improvements are set in place aiming at strengthening commitment control and budget execution. Among several initiatives, the establishment of General Directorates of Financial Services and the extension of commitment registers to the National Health Service are key measures to stem the creation of arrears. Another important reform, aimed at effective budget execution, is the introduction of a new rule that would impose automatic cuts in entities' expenditures (other than wages, pensions and social benefits) whenever expenditure targets are missed due to non-cyclical factors.

\section{The privatization program}

The government is committed to energize the privatization program, which effectively stalled in 2012. The government's priorities include:

- Speeding up privatization in the energy sector and the state-owned railways;

- Promoting long-term concessions for regional airports and ports;

- Facilitating strategic and private investment, including the development of the former Athens International Airport site.

The Hellenic Republic Asset Development Fund (HRADF) has made progress with the privatization of six state assets and has started the privatization process of the State Lottery, infrastructure investments, notably port authorities, and real estate assets. Due to both delays in the initiation of the process and the globally unfavorable economic climate, the expected yields from the privatization program will be about $€ 2.6$ billion in 2013, $€ 2.4$ billion in 2014, $€ 1.1$ billion in 2015 and about $€ 3.4$ billion in 2016 . These are below the targets set during the approval of the EFF-supported program, but the government is committed to strengthen the governance of the HRADF, which will additionally remain fully accountable to parliament on an ex-post basis for the integrity of every privatization sale. 


\section{Bank recapitalization and restructuring}

The banking sector has benefitted greatly from financial assistance under the Fundsupported program, following the adverse effects of the PSI on its balance sheets. The authorities are determined to enhance confidence in the banking system, by creating a leaner, well-capitalized and viable banking sector. The completion of this process would be the first step towards encouraging repatriation of deposits to Greek banks and reversing the adverse financing conditions for enterprises and households.

As part of the ongoing restructuring efforts, in July the Agricultural Bank, a large stateowned bank, was merged with Piraeus Bank, a private bank. In October the acquisition of Emporiki bank by Alpha Bank, and of Geniki Bank by Piraeus Bank were also agreed. In addition, the National Bank of Greece, the largest commercial financial institution, has offered a voluntary share swap to Eurobank, the second largest commercial bank in Greece, aiming to create the largest regional bank in South East Europe. Looking forward, the Greek government and the Bank of Greece have established a framework to ensure continued restructuring and strengthening of the banking system after the recapitalization process is complete.

\section{E. Attracting foreign investment}

Since March the government has been making a strong effort to encourage growthpromoting policies. The rate of absorption of EU structural funds has been constantly increasing and in the period up to May 2012 it was above the EU average for the first time. Most priority projects, whose cumulative budget through end-2015 is nearly 6 percent of GDP, are on schedule. In support of SME development, in July and September the Greek government signed agreements with the European Investment Bank concerning the operation of a Guarantee Fund, which will support lending to small and medium enterprises, and the deployment of a Risk-Sharing Instrument aimed to support infrastructure projects and investments in the energy sector.

\section{F. Liberalization of Labor, Product and Services markets}

While labor reforms took center stage early in the Fund-supported program, reforms in other areas were considerably delayed in 2011 and early 2012. Now the Greek government is determined to forge ahead with further reforms in the labor market and to initiate fundamental reforms in the product and services markets. In particular:

\section{- Strengthening labor market institutions.}

Following recent reductions in severance pay, the government will promote an efficient wage-setting mechanism, reduce non-wage labor costs (e.g. through steps to reduce the administrative burden posed by various regulations of the Labor Inspectorate), and create more options for the adaptability of working hours, especially for small- and mediumscale enterprises. These reforms will further restore cost-competitiveness and boost employment over the medium term. 
- Promoting an efficient and competitive business environment

Building on the improvement in the Doing Business in Greece ranking noted earlier, a new "road map" released in November reduces the administrative burden of (a) creating a company, (b) obtaining licenses for manufacturing activities, and (c) setting-up import and export companies. These measures are accompanied by corresponding initiatives to improve the functioning of the judicial system.

\section{- Liberalizing energy policy}

The government is designing a comprehensive energy policy, the main pillars of which are (a) a restructuring of the Public Power Company, (b) reforming the renewable energy support schemes, (c) introducing measures to have effective competition in generation and supply of electricity, and (d) facilitating the import and trade of oil and oil products.

\section{- Reforming transport services}

Government priorities reflect measures to (a) remove restrictions in limousine and shuttle services, (b) improve the operation and connectivity of ports, (c) restructure the Civil Aviation Authority, and (d) gradually restructure public railways with the aim of eventual privatization. The comprehensive reform of transport services is expected to reduce operating costs of service providers while increasing consumers' choice.

\section{- Liberalizing "regulated" professions}

For many years "protected" or "regulated" professions in Greece led to inefficient practices and oligopolistic pricing at the expense of consumer choice. Under a comprehensive plan of liberalization, several "regulated" professions have been reformed, including the elimination of mandatory use of services, the streamlining of minimum fees and the simplification of recognition of professional qualifications. Such initiatives are expected to both increase the supply of services and enhance their quality and effectiveness.

\section{Conclusion}

The current economic crisis has brought about severe economic and social dislocations, the effects of which are without precedent in modern Greek history. But with a challenge comes an opportunity, and the Greek authorities are resolved to change the economy's structure and orient it towards high-productivity sectors, which will produce internationally tradable goods and services that would be competitive in both the domestic and the external markets. The crisis is also a chance to modernize public administration by reducing the size of the public sector, while at the same time rendering it more efficient and more business- and citizen-friendly.

The Greek authorities broadly agree with the staff analysis, recommendations and appraisal, and look forward to continuing the close cooperation with the Fund staff; they have asked me to convey their deep appreciation for the policy advice, the constructive dialogue and the extensive technical assistance that have been provided in the past two- 
and-a-half years. With the completion of all prior actions, the introduction of correction mechanisms to safeguard the achievement of the fiscal targets, and, more generally, the enhancement of governance of the program that will help it remain on track, my authorities are confident that Greece is about to turn a new page on the long and arduous road towards growth and broad-based economic prosperity. Although the Government is fully aware of the risks involved in this major endeavor, no less because it will strain the capacity of an already burdened civil service, it is also hopeful that 2013 will be the year when the sacrifices of the Greek people will begin to bear fruit. In support of the continuing efforts by the government and the people of Greece, my authorities are requesting the Board's approval of the completion of the first and second reviews and associated waiver, modification of performance criteria and rephasing of access of the EFF-supported program. 